Pontifícia Universidade Católica $_{\text {mato }}$

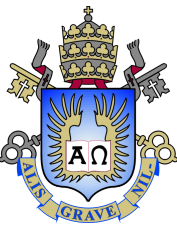

Andre de Queiroz Brunelli

Three Essays on Macroeconomics

Tese de Doutorado

Thesis presented to the Programa de Pós-graduação em Economia of PUC-Rio in partial fulfillment of the requirements for the degree of Doutor em Economia.

Advisor: Prof. Carlos Viana de Carvalho 


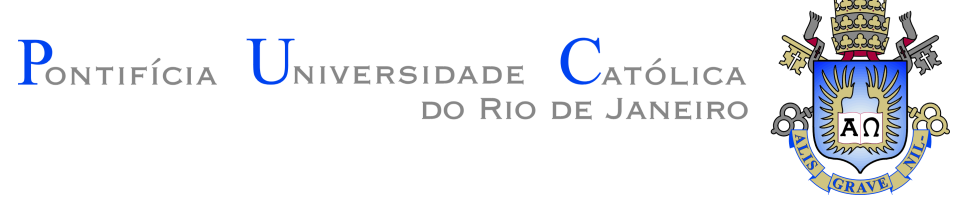

Andre de Queiroz Brunelli

\section{Three Essays on Macroeconomics}

Thesis presented to the Programa de Pós-graduação em Economia of PUC-Rio in partial fulfillment of the requirements for the degree of Doutor em Economia. Approved by the Examination Committee.

Prof. Carlos Viana de Carvalho

Advisor

Departamento de Economia - PUC-Rio

Prof. Eduardo Zilberman

Departamento de Economia - PUC-Rio

Prof. Cézar Augusto Ramos Santos

Fundação Getúlio Vargas - Matriz

Prof. Felipe Saraiva Iachan

EPGE - FGV

Prof. Marco Bonomo

Instituto de Ensino e Pesquisa - Insper

Rio de Janeiro, September 6th, 2019 
All rights reserved.

\section{Andre de Queiroz Brunelli}

Completed his Bachelor of Arts degree in Economics from Universidade Federal do Rio de Janeiro in 2005 and obtained his Master of Science degree in Economics from University College London in 2012. Now holds a PhD degree in Economics from PUC-Rio.

Bibliographic data

de Queiroz Brunelli, Andre

Three Essays on Macroeconomics / Andre de Queiroz Brunelli; advisor: Carlos Viana de Carvalho. - Rio de Janeiro: PUC-Rio, Departamento de Economia, 2019.

206 f: il. color. ; $29,7 \mathrm{~cm}$

Tese (doutorado) - Pontifícia Universidade Católica do Rio de Janeiro, Departamento de Economia, 2019.

Inclui bibliografia.

1. Economia - Teses. 2. Transformação Estrutural;. 3. Desigualdade de Consumo;. 4. Sistemas de Despesas Lineares;. 5. Efeito de Preço;. 6. Efeito de Renda;. 7. Stone-Geary;. 8. Consumo;. 9. Dívida;. 10. Hipótese de Renda Permanente;. 11. Heterogeneidade;. 12. Restrições de Liquidez. I. de Carvalho, Carlos Vianna. II. Pontifícia Universidade Católica do Rio de Janeiro. Departamento de Economia. III. Título. 


\section{Acknowledgments}

I would like to express my gratitude to my advisor Carlos for his guidance, encouragement and motivation. Surely one of the most enthusiastic professionals I have ever met;

I am indebted to Eduardo Zilberman for valuable ideas and for believing in my projects. I also thank Bernardo Ribeiro and all professors in my examination committee for their suggestions;

Central Bank of Brazil and PUC are gratefully acknowledged for sponsoring my $\mathrm{PhD}$ program. I am also thankfull for all employees, professors and colleagues in such an special place which is the Economics Department at PUC-Rio;

This study was financed in part by the Coordenação de Aperfeiçoamento de Pessoal de Nível Superior - Brasil (CAPES) - Finance Code 001;

I thank my family for their unconditional support and, finally, I would like to express my deepest gratetude to my wife Tatiana for all the help, companionship and for the most precious achievement of our lives, our daughter Sara. 


\section{Abstract}

de Queiroz Brunelli, Andre; de Carvalho, Carlos Vianna (Advisor). Three Essays on Macroeconomics. Rio de Janeiro, 2019. 206p. Tese de doutorado - Departamento de Economia, Pontifícia Universidade Católica do Rio de Janeiro.

This thesis is comprised of three essays. The first two investigate the relationship between households per capita income and sectoral expenditure shares both in times series and in cross-section in the postwar US. The first uses a partial approach to estimate the rise of consumption (income) dispersion and income effects in the US from 1980 to 2010. We show that income effects are heterogeneous across households grouped by income quintiles and then consumption dispersion correlates the two main driving forces of structural change (price and income effects) in accounting for the magnitude of structural change in the shares of consumption expenditure in the US over this period. The second extends a canonical Bewley-Aiyagari model in continuous time embedded with a two-sector environment to depict quantitatively three empirical regularities in the postwar US (relative price of goods falls and expenditure shares of goods falls systematically with per capita income, both in times series and in cross-section) without departing from benchmark StoneGeary preferences. We assess the importance of changes in income and relative prices for structural change in the shares of consumption expenditure in the postwar US and conclude they are nearly equivalent forces. We reinforce that reconciling these three main empirical regularities in the postwar US calls for a growth theory that accommodates long-run demand and supply drivers of structural change. Finally, the third essay uses a unique panel dataset with individual-level administrative records of credit transactions, program benefits, individual demographics and features of labor contracts to study how consumers respond to a liquidity shock arising from withdrawals releases from inactive accounts of the Guarantee Fund for Time of Service (FGTS) in Brazil in 2017. Using a difference-in-differences identification design, we find consumption rose and total debt declined after the announcement: during up to twelve subsequent months, for each $\$ 1$ of program benefit, consumers on average increased consumption spending by $\$ 0.53-25$ percent of which occurs during the announcement window - and total debt declined by $\$$ 0.07, specially in payroll debt. Consumption response occurred mostly via 
credit card spending, but evidence of debt-financed durables was also found. Indebted consumers used short-term liquidity in debt modalities (overdraft debt and credit card debt) in addition to credit card spending to smooth consumption. Constrained consumers, measured as young or old, showed stronger consumption responses.

\section{Keywords}

Structural Change; Consumption Inequality,; Linear Expenditure Systems; Price Effect; Income Effect; Stone-Geary; Consumption; Debt; Permanent Income Hypothesis; Heterogeneity; Liquidity Constraints 


\section{Resumo}

de Queiroz Brunelli, Andre; de Carvalho, Carlos Vianna. Três Ensaios em Macroeconomia. Rio de Janeiro, 2019. 206p. Tese de Doutorado - Departamento de Economia, Pontifícia Universidade Católica do Rio de Janeiro.

Esta tese é composta por três ensaios. Os dois primeiros investigam a relação entre a renda per capita das famílias e as frações dos gastos setoriais, tanto em séries temporais quanto em cross-section nos EUA do pós-guerra. O primeiro usa uma abordagem parcial para estimar o aumento da dispersão do consumo (renda) e os efeitos de renda nos EUA de 1980 a 2010. Mostramos que os efeitos da renda são heterogêneos entre as famílias agrupadas por quintis de renda e, em seguida, a dispersão do consumo é correlacionada com as duas principais forças de transformação estrutural (efeitos de preço e renda) na contabilização da magnitude de transformação estrutural nas partes das despesas de consumo nos EUA durante esse período. O segundo estende um modelo canônico de Bewley-Aiyagari em tempo contínuo incorporado a um ambiente de dois setores para representar quantitativamente três regularidades empíricas nos EUA do pós-guerra (o preço relativo dos bens cai e a parcela de gastos dos produtos cai sistematicamente com a renda per capita, tanto em séries temporais quanto no cross-section) sem se afastar das preferências padrão Stone-Geary. Avaliamos a importância de mudanças na renda e nos preços relativos para mudanças estruturais nas parcelas dos gastos de consumo nos EUA do pós-guerra e concluímos que são forças equivalentes. Reforçamos que a conciliação dessas três principais regularidades empíricas nos EUA do pós-guerra exige uma teoria do crescimento que acomode a demanda de longo prazo e forneça fatores de mudança estrutural. Finalmente, o terceiro ensaio usa um conjunto de dados de painel exclusivo com registros administrativos em nível individual de transações de crédito, benefícios do programa, demografia individual e características de contratos de trabalho para estudar como os consumidores respondem a um choque de liquidez decorrente de liberações de saques de contas inativas do Fundo de Garantia por tempo de serviço (FGTS) no Brasil em 2017. Usando um design de identificação de diferenças entre diferenças, encontramos um aumento no consumo e uma dívida total diminuída após o anúncio: durante até doze meses subsequentes, para cada US\$ 1 de benefício do programa, os consumidores a média aumentaram os 
gastos de consumo em US\$ 0,53 - 25\% dos quais ocorrem durante a janela de anúncio - e a dívida total diminuiu em US\$ 0,07, especialmente em dívidas de folha de pagamento. A resposta ao consumo ocorreu principalmente por meio de gastos com cartão de crédito, mas também foram encontradas evidências de bens duráveis financiados por dívida. Os consumidores endividados usaram liquidez de curto prazo nas modalidades de dívida (cheque especial e dívida com cartão de crédito), além dos gastos com cartão de crédito para suavizar consumo. Consumidores restritos, medidos como jovens ou idosos, mostraram respostas mais fortes ao consumo.

\section{Palavras-chave}

Transformação Estrutural; Desigualdade de Consumo; Sistemas de Despesas Lineares; Efeito de Preço; Efeito de Renda; Stone-Geary; Consumo; Dívida; Hipótese de Renda Permanente; Heterogeneidade; Restrições de Liquidez 


\section{Table of Contents}

Chapter 1 Consumption Inequality and Structural Change: A Partial Equilibrium Approach 15

$\begin{array}{lll}1.1 & \text { Introduction } & 15\end{array}$

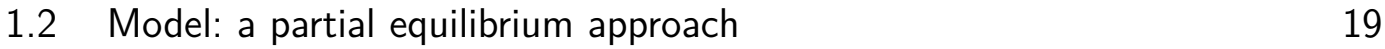

1.3 Implementing the Weighted-average Aggregate Sectoral Expenditure Shares 22

1.3.1 Extensive Margin of Consumption Expenditure: quintiles' expenditure weights 23

1.3.1.1 Data 23

$\begin{array}{lll}\text { 1.3.1.2 Estimation } & 24\end{array}$

$\begin{array}{lll}\text { 1.3.1.3 Estimation Results } & 31\end{array}$

1.3.2 Intensive Margin of Consumption Expenditure: quintiles' sectoral expenditure shares 34

1.3.2.1 Data 34

$\begin{array}{ll}\text { 1.3.2.2 Estimation } & 40\end{array}$

$\begin{array}{lll}\text { 1.3.2.3 Estimation Results } & 42\end{array}$

1.4 Income Effects, Consumption Inequality and Structural Change $\quad 47$

1.4.1 Relevance of Income Effects of the Structural Change 47

1.4.2 Counterfactual Experiment 52

1.5 Conclusion $\quad 58$

Chapter 2 Reconciling Kuznets Facts, Engel's Law and Relative Prices trend in the postwar US in a Bewley-Aiyagari Framework with Stone-Geary Preferences $\quad 60$

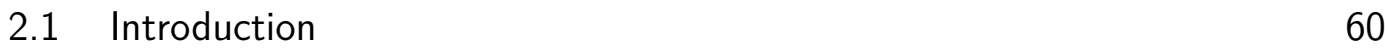

2.2 Model Setup 66

2.2.1 Household Preferences 66

$\begin{array}{ll}2.2 .2 \text { Technology } & 70\end{array}$

$\begin{array}{ll}\text { 2.2.3 Aggregator and Market Clearing } & 71\end{array}$

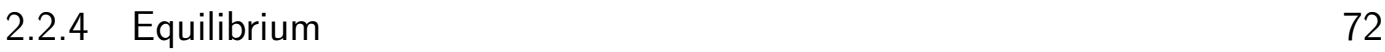

2.2.4.1 Transition dynamics $\quad 72$

$\begin{array}{ll}\text { 2.2.4.2 Stationary equilibrium } & 75\end{array}$

2.3 Numerical Simulation $\quad 77$

2.3.1 Data and Model Calibration $\quad 79$

2.3.2 Results 84

2.3.2.1 Intertemporal opportunities and sectoral dynamics 84

2.3.2.2 Fact i: Relative price of goods 88

2.3.2.3 Fact ii: Kuznets facts $\quad 89$

2.3.2.4 Fact iii: Engel's law 91

$\begin{array}{lll}2.4 & \text { Conclusion } & 99\end{array}$

Chapter 3 Follow the Money: The Effects of Liquidity Shocks on Consumption and Debt - Evidence from a Natural Experiment in Brazil 
$\begin{array}{lll}3.1 & \text { Introduction } & 102\end{array}$

$\begin{array}{ll}\text { 3.2 Withdrawals Program of the FGTS } & 107\end{array}$

3.3 Data and Methodology 109

$\begin{array}{lll}\text { 3.3.1 Data } & 109\end{array}$

$\begin{array}{ll}\text { 3.3.2 Methodology } & 115\end{array}$

$\begin{array}{lll}3.4 \text { Results } & 118\end{array}$

3.4.1 Matched Sample Analysis 118

$\begin{array}{lll}3.4 .2 & \text { Full Sample Analysis } & 121\end{array}$

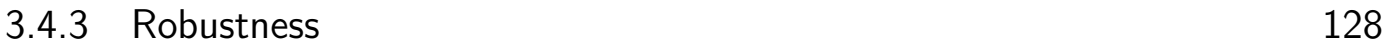

$\begin{array}{ll}3.5 \text { Conclusion } & 129\end{array}$

$\begin{array}{ll}\text { Bibliography } & 153\end{array}$

$\begin{array}{lll}\text { Appendix A } & \text { Chapter } 1 & 158\end{array}$

$\begin{array}{lll}\text { A.1 Additional Tables and Figures } & 158\end{array}$

A.2 Aggregation of Demand Functions 175

A.3 Linear Expenditure Systems of the Extensive and Intensive Margins of Consumption Expenditure 179

Appendix B Chapter 2 181

B.1 Additional Tables and Figures 181

B.2 Proofs and derivations 184

B.3 Data 190

B.3.1 NIPA-BEA data 190

B.3.2 CE-BLS data 190

B.4 Implementation of the estimation of Stone-Geary preferences parameters 192

$\begin{array}{lr}\text { Appendix C Chapter 3 } & 197\end{array}$

C.1 Additional Tables 198

C.2 A simple model of wealthy hand-to-mouth behaviour under an unexpected liquidity shock to the FGTS illiquid assets 202

C.2.1 Consumption decision at $t=1$ : no-borrowing case 203

C.2.2 Consumption decision at $t=1$ with an unexpected liquidity shock: 203

C.2.3 Consumption decision at $t=1$ with borrowing constraint 204

C.2.4 Consumption decision at $t=1$ with an unexpected liquidity shock: 205 


\section{List of Figures}

Figure 1.1 Trends in Aggregate Sectoral Shares - Consumption Expenditure 17

Figure 1.2 Trends of the Quintiles' Expenditure Weights in Total Expendi-

ture Computed From Reported Data in the CE Survey and Constructed

From the Second-stage Regressions (Corrected Data) 34

Figure 1.3 Sectoral Price Indices 38

Figure 1.4 Sectoral Quantity Indexes by Income Quintiles 39

Figure 1.5 Trends in Sectoral Expenditure Shares by Income Quintiles 40

Figure 1.6 Fit of the Model: Expenditure Shares by Income Quintiles 45

Figure 1.7 Fit of the Model: Expenditure Shares on Aggregate Data and

Weighted-average Aggregate Expenditure Shares 46

Figure 1.8 Trends in Sectoral Expenditure Elasticities by Income Quintiles 48

Figure 1.9 Weighted-average Aggregate Expenditure Shares and Income and Price Effects $\quad 50$

Figure 1.10 Weighted-average Aggregate Expenditure Shares and Income

Effects by Selected Income Quintiles 51

Figure 1.11 Counterfactual experiment: No Increase in Consumption Inequality 53

Figure 1.12 Trends in Consumption Inequality Based on Relative Expenditure Patterns Obtained From Reported Data in the CE Survey and Obtained From the Second-stage Regressions

Figure 2.1 Kuznets Facts and Engel's Law in the Postwar US 61

Figure 2.2 Goods to Services Prices Ratio in the Postwar US 62

Figure 2.3 Transition Dynamics of Aggregate Total Expenditure, Aggregate

Capital Stock, Interest Rate and composite consumption Price

Figure 2.4 Average Savings in Selected Periods of the Transition Dynamics 87

Figure 2.5 Fit of the Model to Goods to Services Prices Ratio in the Postwar

Figure 2.6 Fit of the Model to Kuznets Facts in the Postwar US

Figure 2.7 Engel's Law in the in the US - Predictions of the Model 1986-2010

Figure 2.8 Engel's Law in the in the US According to Reported Expenditure in CE-BLS -1986-2010

Figure 2.9 Fit of the Model to Goods to Services Prices Ratio in the Postwar US

Figure 2.10 Fit of the Extended Model to Kuznets Facts in the Postwar US

Figure 2.11 Engel's Law in the in the US Predicted by the Extended Baseline

Model and According to Reported Nondurable Expenditure in CE-BLS $-1986-2010$

Figure 3.1 Web Searches for "FGTS saques" on Google Trends 131

Figure 3.2 Kernel Densities of the Matched Sample - Age 136

Figure 3.3 Kernel Densities of the Matched Sample - Monthly Income in 2016

Figure 3.4 Kernel Densities of the Matched Sample - Monthly Credit Card Spending in 2016 
Figure 3.5 Unconditional Mean of Consumption 139

Figure 3.6 Unconditional Mean of Total Debt 140

Figure 3.7 Heterogeneity in Consumption Response across Workers Types

-Age 152

Figure A.1 Trends of the Quintiles' Expenditure Weights in Total 161

Figure A.2 Trends in Aggregate Sectoral Shares - Consumption Expenditure 163

Figure A.3 Trends in Sectoral Price Indexes - Consumption Expenditure 165

Figure A.4 Trends in Sectoral Quantity Indexes - Consumption Expenditure 166

Figure A.5 Sectoral Quantity Indexes by Income Quintiles - Nondurable 167

Figure A.6 Trends in Sectoral Expenditure Shares by Income Quintiles 168

Figure A.7 Trends in Goods Expenditure Shares by Income Quintiles 169

Figure A.8 Fit of the Model: Expenditure Shares by Income Quintiles 171

Figure A.9 Fit of the Model: Expenditure Shares on Aggregate Data and

Weighted-average Aggregate Expenditure Shares 172

Figure A.10 Counterfactual experiment: No Increase in Consumption 173

Figure B.1 Transition Dynamics of Aggregate composite consumption 181

Figure B.2 Savings Policy in Selected Periods of the Transition Dynamics 182

Figure B.3 Savings Policy in Selected Periods of the Transition Dynamics 182

Figure B.4 Savings Policy in Selected Periods of the Transition Dynamics 183

Figure B.5 Density in the New Stationary Equilibrium 183

Figure B.6 Engel's Law According to Brazilian Data 184 


\section{List of Tables}

Table 1.1 Trends in Inequality: Log Change of the Ratio of each Income Quintile to Bottom Income Quintile

Table 1.2 Trends in Consumption Inequality Based on Relative Expenditure Patterns and Trends in Income Inequality

Table 1.3 Association of Broad Consumption Sectors, CE Consumption Categories and CPI Items

Table 1.4 Sectoral Share Estimation Results for the Aggregate and Income Quintiles $\left(q_{1}-q_{5}\right)$

Table 1.5 Percent Change of the Variation of the Weighted-average Aggregate Expenditure Shares Attributed to the Correlation With the Increase of Consumption Inequality - 2008-2010/1983-1985

Table 2.1 Model Calibration - First Part

Table 2.2 Model Calibration - Second Part

Table 2.3 Targeted Moments - Nonhomotheticity Term of Goods Relative

To Final Consumption Expenditure

Table 3.1 Summary Statistics - Demographics

Table 3.2 Summary Statistics - Consumption and Debt

Table 3.3 The Average Consumption Response to the FGTS Program 141

Table 3.4 The Average Total Debt Response to the FGTS Program 142

Table 3.5 The Consumption Response Dynamics 143

Table 3.6 The Total Debt Response Dynamics 144

Table 3.7 The Average Consumption Response to the FGTS Program 145

Table 3.8 The Average Total Debt Response to the FGTS Program 146

Table 3.9 The Consumption Response Dynamics 147

Table 3.10 The Total Debt Response Dynamics 148

Table 3.11 Credit Card Spending, Payroll Debt and Overdraft Debt Dynamics Responses - Restricted Sample

Table 3.12 Response heterogeneity by consumption categories and debt modalities

Table A.1 Trends in Inequality: Log Change of the Ratio of each Income 158

Table A.2 Consumption Expenditure Shares and First-Stage Expenditure 159

Table A.3 Trends in Consumption Inequality Based on Relative Expenditure 160

Table A.4 Change In Relative Income-Specific Measurement Error 162

Table A.5 Association of Broad Consumption Sectors, CE Consumption 164

Table A.6 Sectoral Share Estimation Results for the Aggregate and Income Quintiles $\left(q_{1}-q_{5}\right)$-Nondurable Expenditure 170

Table A.7 Percent Change of the Variation of the Weighted-average 174

Table B.1 Estimation Results with Final Consumption Expenditure Baseline model and Extended Baseline Model

Table B.2 Targeted Moments - Nonhomotheticity Term of Nondurable Goods Relative To Final Nondurable Consumption Expenditure 
Table C.1 Propensity Score Matching Logistic Regressions

Table C.2 Credit Card Spending, Payroll Debt and Credit Card Debt Dynamics Responses - Restricted Sample

Table C.3 Robustness - Cross Sectional Test in the Matched and Full Samples

Table C.4 Consumption Allocation in $t=1, c_{1}$, and Liquid Asset Allocation in $t=2, m_{2}$, in Model Cases in Appendix C 


\section{Chapter 1 \\ Consumption Inequality and Structural Change: A Partial Equilibrium Approach}

\section{1 \\ Introduction}

Sectoral reallocation of aggregate variables is a well documented feature of economic development. As income grows through time, value added, employment, and final consumption expenditure move gradually from agriculture to manufaturing. Then this reallocation process - widely known as structural change (transformation) - continues further from these broad goods sectors (agriculture and manufacturing) to the services sector, which ultimately becomes the largest sector (Fisher (1939), Clark (1940), Kuznets (1955), Chenery (1960),Maddison (1980)). Lately, a similar pattern was documented in the cross-section of the households expenditure (Boppart, 2014), which reinforces the structural change as one of the main stylized facts of economic development.

Recent work on the literature of structural change extends the standard one-sector growth model to incorporate either supply or demand mechanisms to explain this dynamic process through two main driving forces: changes in relative sectoral prices (price effects) - supply side theories - and changes in aggregate income (income effects) - demand side theories. The former stresses in general two mechanisms behind relative prices changes: heterogeneous growth of sectoral technology (Baumol (1967),Ngai and Pissarides (2007)) and differences in sectoral capital intensities (Acemoglu and Guerrieri, 2006). The latter, in turn, focuses on the income (expenditure) elasticity mechanism - the relation between income growth and nonhomotheticity in preferences which engender persistent differences in the slope of Engel curves of broad sectors ${ }^{1}$ (Kongsamut et al. (1997),Foellmi and ZweimÃijller (2008))

Even though this literature has already solidified the dynamic process through which output growth imply structural change and despite any further dispute on its driving forces, little is known in terms of whether consumption

\footnotetext{
${ }^{1}$ Broad sector in the literature of structural change in general means either goods (agriculture and manufacturing) and services sectors or the goods sector is split between agriculture and manufacturing sectors in addition to the services sector.
} 
(and income) dispersion concerns structural change. Moreover, there is a relatively consolidated interconnection of structural change with several macroeconomic aspects in the literature, including international trade, income convergence, demographics and labor supply. However, even though recent studies in the structural change field feature income and consumption inequality, empirical and theoretical works focused exclusively on studying the relation between consumption inequality and structural change have been limited. For example, in Buera and Kaboski (2009) wage inequality arises endogenously in a model aimed at explaining the rise of the services sector that features human capital so that skill premium is tightly linked to demand patterns associated with income per capita. In Boppart (2014), a macroeconomic model features expenditure inequality as a single sufficient statistic. It enables tractability of a nontrivial aggregation so that it rationalizes aggregate and cross-sectional stylized facts of structural change along with the aggregate Kaldor facts.

Therefore, an important question that arises in this literature is whether consumption inequality and structural change are connected. In this sense, this question can be seen as an application in a broader context that studies, on the one hand, when inequality matters for the dynamics of macroeconomic aggregates and, on the other hand, when macroeconomic shocks and policies affect inequality (see, for example, Ahn et al. (2017)).

In this paper we seek to tackle this question empirically. Rather than identifying an exogenous causal effect of consumption inequality on structural change or to disentangle general equilibrium feedbacks, we take a step back to initially stress a reduced-form of this correlation. We take a partial approach to a model of structural change with disaggregate sectoral expenditure shares specified over US household final consumption expenditure data from the Consumption Expenditure Survey of the Bureau of Labor Statistics (CE-BLS) survey that follows Aguiar and Bils (2011) and price data from the Consumer Price Index (CPI-BLS) over the period 1980 - 2010. The disaggregate sectoral expenditure shares are consistent with aggregate analogues defined by previous work (Herrendorf et al., 2009) applied on aggregate consumption expenditure data from the National Income and Product Accounts of the Bureau of Economic Analysis - NIPA-BEA (See Figure 1.1) for the postwar period ${ }^{2}$.

${ }^{2}$ We use Table 2.4.5 - "Personal Consumption Expenditures by Type of Product". We exclude from total personal consumption expenditure the net foreign travel expenditures, final consumption expenditures of nonprofit institutions serving households and do not include government consumption expenditure for consistency with CE-BLS data. See the data subsection 3.2.1 for further details on definitions. 


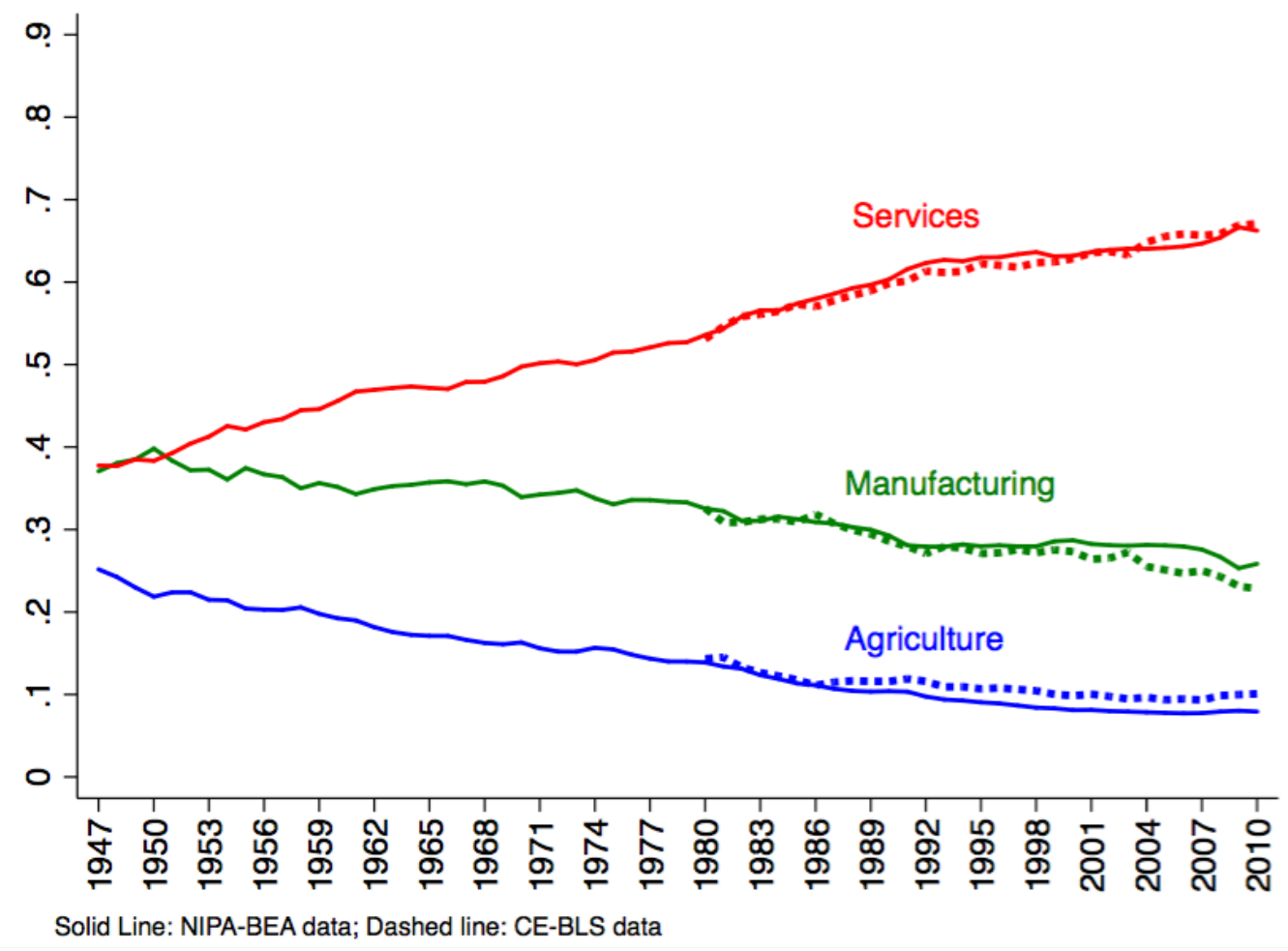

Figure 1.1: Trends in Aggregate Sectoral Shares - Consumption Expenditure

Notes: This figure depicts the approximation of aggregate shares of consumption expenditure using data from CE-BLS and data from NIPA-BEA for agriculture, manufacturing and services that follows (Herrendorf et al., 2009) subject to smaller consistency issues due to data source differences. Solid and dashed lines denote, respectively, data from NIPA-BEA and data from CE-BLS. Sectoral shares are defined by the ratio of sectoral expenditure to total expenditure. See the data subsection 3.2.1 for further details on definitions.

We consider the aggregate sectoral expenditure shares as the composition of two margins of consumption expenditure: an extensive margin - consumption inequality, expressed as the expenditure weights of households grouped by income quintiles - and an intensive margin - income quintiles' sectoral expenditure shares. By doing this, the main contribution of this paper is to estimate an empirical correlation between consumption inequality and structural change. To this respect, we follow basically two steps.

First, we implement a methodology that provides a tractable framework to address systematic mis-measurements of reported expenditures in the $\mathrm{CE}$ survey which follows Aguiar and Bils (2011). Hence, we use a log-linear approximation to the Engel curves to obtain a corrected measure of the change of consumption inequality that is consistent with the change of income inequality $^{3}$ without which reported expenditures would systematically understate consumption inequality so that the correlation between consumption inequal-

${ }^{3}$ For this debate on whether income inequality is mirrored by consumption inequality, see for example, Attanasio et al. (2007), Attanasio and Pistaferri (2014) ; Heathcote et al. (2010) and Aguiar and Bils (2015). 
ity and structural change virtually does not show up empirically. We show that the increase of consumption inequality is driven basically by the top income quintile. Second, we estimate the intensive margin of consumption expenditure - the income quintiles' sectoral expenditure shares - based on a structural analogue of the reduced-form linear expenditure system used to estimate the extensive margin of consumption expenditure, which is a fairly standard demand system that features nonhomotheticity of preferences. We show that income effects are the dominant source of the aggregate structural change in the shares of consumption expenditure, which is in line with other studies that apply aggregate data (see, for example, Herrendorf et al. (2009)). We also highlight that there are heterogeneous income effects across households grouped by income quintiles and that the top income quintile captures the bulk of the changes in the aggregate sectoral expenditure shares. Moreover, agricultural and manufacturing goods are necessities and services are luxuries for the top quintile.

We then use the estimated model to perform a counterfactual experiment to measure the contribution of the rise of consumption inequality to structural change in the US economy in the past three decades. We find that the increase of consumption (income) inequality correlates, although marginally, with the two main driving forces of structural change - changes in relative sectoral prices and, in special, changes in aggregate income - in accounting for the magnitude of structural change in the shares of consumption expenditure in the US. Particularly, we show that without the increase of consumption inequality, the aggregate expenditure share of services would be lower ${ }^{4}$ and, conversely, the aggregate expenditure shares of agriculture and manufacturing would be higher ${ }^{5}$ than the actual ones.

Besides this introduction, this paper is organized as follows. Section 2 presents the model; Section 3 describes the data and discusses the estimation procedure and its results both for the consumption inequality - quintiles's expenditure weights - and for the quintiles' sectoral expenditure shares. In section 4 , we assess the heterogeneity of income effects of structural change in

${ }^{4}$ The rise of aggregate expenditure share of services would be lower by 1.8 percent and 4.4 percent, respectively, by considering consumption expenditure and nondurable expenditure data.

${ }^{5}$ The fall of the aggregate expenditure share of agriculture would be lower by 10.8 percent and 6.2 percent, respectively, by considering consumption expenditure and nondurable expenditure data. The fall of the aggregate expenditure share of manufacturing, in turn, would be higher by 2.3 percent by considering consumption expenditure. However, we argue that in the manufactuing sector a lower contrafactual expenditure share is due to mismeasurement specially associated to durables since if we consider nondurable expenditure, the fall of the aggregate expenditure share of manufacturing would be lower by 2.5 percent. See Table A.7 in Appendix A. 
households grouped by income quintiles and runs the counterfactual experiment. Section 5 concludes.

\section{2}

\section{Model: a partial equilibrium approach}

The basic aim of this paper is to stress whether there is an empirical evidence that income and consumption inequalities underlie the price and income effects associated to structural change. If so, a necessary condition is to assess whether there are different experiences of structural change across households defined over broad consumption sectors. We outline below the model that we take to US data to approach it.

Our intertemporal preferences belong to a class that features (explicit) additively separable intertemporal preferences - CRRA. In this class of models that features additively separable intertemporal preferences, the household may wish to save or to borrow according to the way in which it evaluates present and future needs and this determines how much to allocate to current consumption and in particular to goods, $c_{q_{k}, i, t}$, where $q_{k} \in\left\{q_{1}, q_{2}, q_{3}, q_{4}, q_{5}\right\}$ stands for the income quintiles and $i=\{a, m, s\}$ are indexes that refer to three broad sectors: agriculture, manufacturing and services. Expenditure allocated to these goods is the first stage in a two-stage allocation process the intertemporal and intratemporal problems.

INTERTEMPORAL PROBLEM. We consider an infinitely lived household in each income quintile that observes the sequence of prices and allocates income between the composite consumption good and savings. Household has preferences represented by a utility function of the form ${ }^{6}$ :

$$
\sum_{t=0}^{\infty} \beta^{t} \frac{u\left(c_{q_{k}, a, t}, c_{q_{k}, m, t}, c_{q_{k}, s, t}\right)^{1-\gamma}-1}{1-\gamma}
$$

where $u\left(c_{q_{k}, a, t}, c_{q_{k}, m, t}, c_{q_{k}, s, t}\right) \equiv c_{q_{k}, t}$ is the composite consumption good and $\gamma>0$ is the inverse of intertemporal elasticity of substitution of consumption;

INTRATEMPORAL (STATIC) PROBLEM. To allocate total consumption expenditure in $t$ among the three types of consumption goods:

${ }^{6}$ Time is discrete. However, note that since our focus in on the static problem it is actually irrelevant whether we consider discrete or continuous time. 


$$
\begin{array}{cl}
\max _{\left\{c_{q_{k}, a, t}, c_{q_{k}, m, t}, c_{q_{k}, s, t}\right\}} & c_{q_{k}, t} \\
\sum_{i=a, m, s} p_{i, t} c_{q_{k}, i, t} & =e_{q_{k}, t},
\end{array}
$$

where: i. $c_{q_{k}, t}=\sum_{i=a, m, s} \varphi_{q_{k}, i} \log \left(c_{q_{k}, i, t}+\bar{c}_{q_{k}, i}\right)$. We use preferences similar to Kongsamut et al. (2001). This utility function was first introduced by Stone (1954) and Geary (1950) and the implied demand model is often called the Linear Expenditure System ${ }^{7}$; ii. $\varphi_{q_{k}, i}$ are nonnegative weights (add up to one); iii. $\bar{c}_{q_{k}, i}$ are constants, such that $\bar{c}_{q_{k}, m}$ is restrict to be zero (Kongsamut et al., 2001; Herrendorf et al. (2009) ), $\bar{c}_{q_{k}, a}<0$ and $\bar{c}_{q_{k}, s}>0$ can be interpreted, respectively, as food subsistence and home-services endowment ${ }^{8}$; iv. $p_{i, t}$ are sectoral goods prices and v. $e_{q_{k}, t}=p_{t} c_{q_{k}, t}-\sum_{i=a, m, s} p_{i, t} \bar{c}_{q_{k}, i}$ denotes total expenditure of quintile $q_{k}$.

We assume that all households have preferences of the above form. If consumption expenditure exceeds a minimum level, then aggregate expenditures are consistent with those for a stand-in household for each income quintile with preferences of the same form. Note that to suppose a stand-in household for each income quintile with Stone-Geary preferences in our partial approach is trivially consistent with a stand-in household for the aggregate economy. ${ }^{9}$

We consider the stand-in household in a setting in which it maximizes lifetime utility given a market structure that features markets for each of the three consumptions goods and a market for borrowing and lending at each date $t$. We follow Herrendorf et al. (2009) in focusing solely on the implications for optimal consumption behavior within each period. This partial equilibrium approach implies that we do not take a stand on the exact nature of intertemporal opportunities available to the household or to specify how expectations of the future are formed. Additionally assuming interior

${ }^{7}$ We select a linear expenditure system for basically two reasons. First, as we shall see, it represents the expenditure patterns in our sample data quite reasonably and, as mentioned, there are several empirical work giving support to linear expenditure systems as good approximations to broad sectors. Second, we can conveniently benefit from Aguiar and Bils (2015)'s methodology to correct for systematic measurement error in consumption expenditure reported in the CE Survey, which is further discussed in subsection 3.1.

${ }^{8} \bar{c}_{q_{k}, s}$ can be negative. However, to interpret it in our environment is tricky. Our interpretation follows Moro et al. (2017). Following these authors, $\bar{c}_{q_{k}, s}<0$ can be interpreted as a minimum requirement of home-services production and traditional market services consumption (for instance household maintenance and cleaning) that the household has to provide/consume before enjoying the rest of home-services and traditional market services. See the section on empirical implementation for further discussion on this matter.

${ }^{9}$ See the Online Appendix A in Herrendorf et al. (2013) for the precise formal condition for a stand-in household in the aggregate economy and Appendix B in our study for the decomposition among income quintiles. This property extends to settings in which individuals make consumption-savings decisions in complete markets. 
solutions ${ }^{10}$, the first order conditions and some algebra yield the following consumption expenditure shares ${ }^{11}$ :

$$
s_{q_{k}, i, t} \equiv \frac{p_{i, t} c_{q_{k}, i, t}}{e_{q_{k}, t}}=\varphi_{q_{k}, i}\left(1+\sum_{g=a, m, s} \frac{p_{g, t} \bar{c}_{q_{k}, g}}{e_{q_{k}, t}}\right)-\frac{p_{i, t} \bar{c}_{q_{k}, i}}{e_{q_{k}, t}},
$$

where we refer to $g=\{a, m, s\}$ here with a slight abuse of terminology.

Finally, the aggregate sectoral expenditure shares are obtained by averaging the quintiles' expenditure shares by expenditure weights of each income quintile in total expenditure:

$$
s_{i, t} \equiv \sum_{k=1}^{5} \omega_{q_{k}, t} s_{q_{k}, i, t},
$$

where $\omega_{q_{k}, t} \equiv \frac{e_{q_{k}, t}}{\sum_{k=1}^{5} e_{q_{k}, t}}$ is the expenditure weight of income quintile $q_{k}$ in total expenditure at time $t$.

Note that the aggregate sectoral expenditure shares can naturally be interpreted as the composition of an extensive margin of consumption expenditure - quintiles' expenditure weights - and an intensive margin of consumption expenditure - quintiles' sectoral expenditure shares. As we shall see, this weighted-average aggregate sectoral expenditure share is key for our results since it enables to connect consumption inequality and structural change through heterogeneous experiences in both margins.

Therefore, rather than being only a decomposition, under certain conditions on demand patterns, structural change expressed by aggregate sectoral expenditure shares can be thought, at least theoretically in our partial approach, as a result of developments arising exclusively from one of the two expenditure margins. That is, developments of the aggregate sectoral expenditure shares might be the result of changes only in the intensive margin, whereas consumption inequality remais constant or, alternatively, it might be exclusively the result of changes in consumption inequality.However, regardless of any theoretical possibility, ultimately this is an empirical question that we seek to tackle in the next section.

\footnotetext{
${ }^{10}$ In general the nonhomotheticity terms in this class of utility functions can lead to corner solutions. However, as argues Herrendorf et al. (2009), this is not relevant for aggregate consumption in a rich country such as the postwar United States and even less relevant particularly after the 1980's. See online Appendix B for the formal condition regarding interior solutions.

${ }^{11}$ Assuming that the utility function as well as the expenditure function feature standard conditions (they are taken to be continuous, diffentiable, increasing and strictly concave) and that there is no corner solution, so that the dual problem applies, to solve problem (1) - (2) by maximizing the composite consumption good in $u\left(c_{q_{k}, t}\right) \equiv c_{q_{k}, t}$ for $c_{q_{k}, i, t}$ subject to total expenditure, $\sum_{I} p_{i, t} c_{q_{k}, i, t} \leq p_{t} c_{q_{k}, t}+p_{a, t} \bar{c}_{q_{k}, a}-p_{s, t} \bar{c}_{q_{k}, s}$, is equivalent to solve it by minimizing total expenditure, $\sum_{I} p_{i, t} c_{q_{k}, i, t} \equiv e_{t}$, for $c_{q_{k}, i, t}$ subject to a utility level $u\left(c_{q_{k}, t}\right) \geq \bar{u}_{q_{k}}$. In this case, marshallian demands arise from Roy's identity.
} 


\section{3}

\section{Implementing the Weighted-average Aggregate Sectoral Expenditure Shares}

This section describes the data and estimation apart into the extensive and intensive margins of consumption expenditure for the United States, as we decompose the aggregate sectoral expenditure shares in equation (4).

Note that, even though we apply an empirical analysis to a specific period in the US, the relative importance of each consumption margin to structural change is conditional to features of development observed in a particular economy, including income growth, inequality and demand patterns associated to income per capita. For example, we can think of two extreme hypothetical examples to illustrate the intuiton underlying this relative importance. First, consider that an stagnant economy for a long period, so that the sectoral expenditure shares virtually did not changed and, in the meantime, consumption inequality increased. By considering that the aggregate expenditure share of services is increasing in income per capita, whereas the aggregate expenditure share of goods are nonincreasing in income per capita, thus, in this case the extensive margin o consumption would account for most of the structural change observed in that period. Conversely, consider that an economy experimented a positive path of economic growth during a long period, while inequality remained virtually unchanged. Hence, in this case, it is the intensive margin of consumption that would account for most of the structural change observed in this economy. In other words, income (consumption) inequality is an underlying feature of the growth dynamics of income per capita that can potentialize its effect on structural change by considering persistent demand patterns of broad sectors in the cross-section of households ${ }^{12}$. That is, conditional on an increasing path of income per capita, the greater the rise (decline) of inequality, the more (less) the share of services expenditure might soar relative to the shares of agricultural and manufacturing expenditure. Conversily, considering a decreasing path of income per capita, the greater the rise (decline) of inequality, the less (more) the share of services expenditure might decline relative to the shares of agricultural and manufacturing expenditure.

Regardless of the fact on whether the theoretical reasons that underlies the nature of the connection between consumption inequality and structural change may or may not be appropriate, ultimately this is an empirical question that we tackle in this section. In subsection 3.1, we correct for systematic measurement errors in the interviews of the CE-BLS regarding to reported

\footnotetext{
${ }^{12}$ See Figure 1.5 in subsection 3.2 and Figures A6 and A7 in appendix for demand pattens in the cross-section of households grouped by income quintiles that we observe in US data from 1980-2010.
} 
expenditure - and then to the associated quintiles' expenditure weights. In subsection 3.2, we estimate our structural change model applying equation (3) for the sectoral expenditure shares of the income quintiles using data from the CE-BLS and the CPI-BLS. Our approach is based on the final consumption expenditure method (as opposed to the value-added method). Thus, we focus on quintiles' sectoral expenditure shares for our estimations. Further details are provided below.

\subsection{1}

Extensive Margin of Consumption Expenditure: quintiles' expenditure weights

\subsubsection{1}

Data

Our data closely follows Aguiar and Bils (2011) 's consumption expenditure database with two small consistency differences since we apply this database on the estimation of the intensive margin of consumption expenditure (sectoral expenditure shares). ${ }^{13}$

We use a pooled cross section over 31 annual time series aggregated from U.S. quarterly household consumption data covering more than 252,000 households. These data are drawn from the CE-BLS for the years 1980-2010. In the $\mathrm{CE}$, each household is interviewed about their expenditures for up to four consecutive quarters. In one form or another the CE has been the cornerstone of many empirical studies of consumer behavior at the micro level, including, for example, the papers by Cutler and Katz (1992), Krueger and Perri (2002), Blundell et al. (2008) and also recently in studies on structural change applied on micro level, as Boppart (2014).

By following Aguiar and Bils (2011), in terms of sample selection the results reported here refer to a sample of urban households whose head is more than 25 and less than 64 years of age. Moreover, as the authors, we only include households that have: at least full-year of interview coverage, complete income reporter, no expenditure outliers and that range between 5-95 percentile of before-tax income to eliminate outliers and mitigate any time-varying impact of bottom-coding and top-coding. It then implies that the bottom income group contains the 5-20 percentile groups and the top income group contains the 80-

\footnotetext{
${ }^{13}$ It is available online at the AER website. For further details, the current link is https://www.aeaweb.org/articles?id=10.1257/aer.20120599.
} 
95 percentile groups ${ }^{14}$. This results in a final sample of 62,734 households.

The first difference with respect to the original authors' database refers to the fact our demand system for the quintiles' weights estimation is specified over final-good consumption constructed by aggregating consumption items over 22 consumption categories. We use the authors' 20 expenditure categories and divide two of them ("vehicle purchase, leising, insurance" and "furniture and fixtures") in nondurable (services) and durable expenditures because both groups are composed specifically by services and durable (goods) items. Since there are no nondurable goods in these groups, we attribute their nondurable part to services. This enhances the definition of the three-sector demand system (agriculture, manufacturing and services) so that it better matches definitions in Herrendorf et al. (2009) and becomes consistent with the estimation of the intensive margin of consumption expenditure in subsection 3.2.

Note that durable goods differences in expenditure across income quintiles do not necessarily align with differences in durable stocks and associated service flows and therefore it might obliterate cross-sectional differences of consumption patterns that we mean to address. For this reason we also report in Appendix A results using the authors' nondurable measure of expenditure in each category to compose a nondurable version of the consumption sectors.

The second difference from the authors' data regards the fact that we use household per capita expenditure data instead of total household expenditure since our model is specified in per capita terms. It means we average household per capita expenditure by income quintiles when documenting differences across income levels. Apart from the two small differences and this summary, see Aguiar and Bils (2011) for a detailed description of the database.

\subsubsection{2}

\section{Estimation}

This subsection describes de estimation of the extensive margin of consumption expenditure, namely, the quintiles' expenditure weights, $\omega_{q_{k}, t}$, which is our measure of consumption inequality. A central motivation for this estimation regards the fact that there are systematic mis-measurements in reported expenditure in consumption expenditure surveys. For example, one of the main clues that stem from our primary data depicts the growth of consumption inequality inconsistently with the growth of income inequality.

\footnotetext{
${ }^{14}$ Although the income groups in the extreme of the income distribution do not compose exactly an income quintile, we keep calling so henceforth for simplicity. Moreover, for illustrative purposes, the average of annual before-tax income of quintiles $q_{1}-q_{5}$ in per capita terms (and constant 1983 dollars by CPI-U - U.S. city average) follows: 5,305; 9,072; 11,934; 14,$360 ; 17,439$, respectively, in 1983 and 5,$546 ; 10,247 ; 13,025 ; 17,577 ; 25,606$, respectively, in 2010 .
} 
Therefore, to compute quintiles' expenditure weights directly by summing household expenditures on reported data from these surveys is potentially misleading. Addressing this potential measurement error is the focus of this subsection.

Table 1.1 reports this seeming inconsistency between the change of consumption inequality and the change of income inequality over the sample period. It log differenciates the ratio of expenditure by income quintiles with respect to the botton quintile between the averages of 2008-2010 and 1983$1985 .{ }^{15}$ Note that in this case we leave aside the beginning period 1980-1982 for comparative reason since this pooled base period is employed in the first step of the two-step estimation discussed below. Furthermore, we report the same measures over two concepts of income (before-tax and after-tax ${ }^{16}$ ) to compare with the consumption one. ${ }^{17}$

\footnotetext{
${ }^{15}$ We average over multiple years to mitigate year-to-year sampling error. We do not follow Aguiar and Bils (2011) in breaking out the recent recession. Although consumption inequality behaves somewhat differently during this period, to show the log change between 2005-2007 and 2008-2010 separately does not add to our main point here regarding the fact the change in consumption inequality does not mirror the change in income inequality in the whole sample period.

${ }^{16}$ See Aguiar and Bils (2011)'s Online Appendix for details on definition of these types of income.

${ }^{17}$ We also report figures over nondurable expenditures in Table A.1 in Appendix A.
} 
log change 1983-1985/2008-2010

\begin{tabular}{|c|c|c|c|}
\hline $\mathrm{q}_{2}$ & $\mathrm{q}_{3}$ & $\mathrm{q}_{4}$ & $\mathrm{q}_{5}$ \\
\hline 0.02 & -0.01 & 0.00 & 0.04 \\
\hline-0.04 & -0.06 & -0.05 & -0.08 \\
\hline 0.01 & -0.04 & -0.09 & -0.06 \\
\hline 0.03 & 0.01 & 0.05 & 0.11 \\
\hline 0.03 & 0.05 & 0.14 & 0.21 \\
\hline 0.06 & 0.08 & 0.17 & 0.24 \\
\hline
\end{tabular}

Table 1.1: Trends in Inequality: Log Change of the Ratio of each Income Quintile to Bottom Income Quintile

Notes: This table depicts the log difference of the ratio of the average of each quintile of before-tax income respondents (quintile 2-quintile 5: $q_{2}-q_{5}$ ) to the average for bottom quintile respondents where the averages are taken over the pooled years indicated at the head of the table. All variables are converted into constant 1983 dollars by CPI-U - U.S. city average before averaging. Definitions of each series and sample construction are given in the data section.

Two aspects arise most notably from Table 1. First, we see an opposing trend of consumption inequality across services and goods sectors, with increasing consumption inequality in services expenditure and a falling consumption inequality in manufacturing and agriculture expenditure. Note that this evidence is consistent with nonhomothetic demands as we consider in our model, in which relative expenditures are increasingly allocated to services as total expenditure (income) rises. Second, the rise of consumption inequality is much less than that of income. For example, by looking at the log change of the ratio of income quintile $q_{5}$ to quintile $q_{1}$ with respect to consumption expenditure and income, we see a 4 percent rise in consumption expenditure, while before-tax income and the after-tax income register an increase of 21 percent and 24 percent, respectively.

Our goal here is to estimate consumption inequality (and associated quintiles' expenditure weights) and there is some cumulating evidence showing that increasing disparities in income are approximately replicated by increasing disparities in consumption, a fact that is well-documented since the beginning of the 1980's (see, for example, Krueger and Perri (2002);Attanasio et al. 
(2007) ; Heathcote et al., 2010; Attanasio and Pistaferri (2014) and Aguiar and Bils (2011)). To this respect, we particularly apply Aguiar and Bils (2011)'s methodology to correct for mis-measurement in the interview survey of the CE-BLS. There is a map connecting our structural linear demand system that arise from the intratemporal problem represented by equations (1) - (2) and the reduced-form Engel curves these authors take to the data to estimate consumption inequality. See Appendix $\mathrm{C}$ for more details on this map and Aguiar and Bils (2011) for details on the estimation procedure and for a discussion on the identification assumption. Below we outline their methodology for brevity.

The estimation of the consumption inequality, which gives rise to the quintiles' expenditure weights, consists of two steps. Before introducing these steps, the step zero is to assume that consumption expenditure is measured with error. Let $e_{j, t}^{h}$ denotes the reported per capita expenditure of household $h$, $h=1, \ldots, H$ on good $j$, such that $j=1, \ldots, J$ indexes our $J=22$ consumption categories at time $t$. We aggregate $e_{j, t}^{h}$ over the $j$ goods so that $e_{t}^{h}$ denotes total per capita expenditure at time $t$ by household $h, e_{t}^{h}=\sum_{j=1}^{J} e_{j, t}^{h}$. Furthermore, since $e_{j, t}^{h}$ is measured with error, $\left(e_{j, t}^{h}\right)^{*}$ denotes the true expenditure and the degree of mis-measurement depends on time, income quintile and good with the following specification:

$$
e_{j, t}^{h}=\left(e_{j, t}^{h}\right)^{*} \exp \left\{\zeta_{j, t}^{h}\right\}
$$

where $\zeta_{j, t}^{h}=\psi_{j, t}+\phi_{q_{k}, t}+v_{j, t}^{h}$, such that $k=1, \ldots, Q$ denotes the $Q=5$ income quintiles; $\psi_{j, t}$ reflects mis-measurement of consumption good $j$ at time $t$ that is common across respondents ${ }^{18} ; \phi_{q_{k}, t}$ denotes mis-measurement specific to income quintile $q_{k}$ at time $t$ that is common across goods ${ }^{19}$; and $v_{j, t}^{h}$ is the residual good-household specific measurement error ${ }^{20}$. The identifying assumption here is that $v_{j, t}^{h}$ is a classical measurement error so that it is independent of the characteristics of good $j$ and household $h$ at each date $t^{21}$.

In the first step, we estimate the total expenditure elasticities for each good. We estimate a log-linear approximation to the Engel curves. One has to keep in mind that log-linear demand systems are not globally theory consistent Deaton and Muellbauer (1980). Specifically, the "adding up" constraint is not

\footnotetext{
${ }^{18}$ Good $j$ may be either underreported or overreported for all households.

${ }^{19}$ Income quintile $q_{k}$ may either underreports or overreports all expenditure categories.

${ }^{20}$ Good $j$ of household $h$ may be either underreported or overreported.

${ }^{21}$ The mean of $v_{j, t}^{h}$ across households is normalized to be zero for all $t$ without loss of generality (given the presence of $\psi_{j, t}$ and $\phi_{q_{k}, j}$ ).
} 
globally satisfied ${ }^{22}$. A suitable alternative of local approximation for our case that focus on structural change through sectoral expenditure shares could be the popular Almost Ideal Demand System (AIDS), which considers that the share of expenditure on good $j$ is log linear in total expenditure. However, it is not well suited to handle good-specific measurement error $\psi_{j, t}$ in the second step of the estimation, even though it brings informative testing implications of consumer optimization. Log-linear demand systems, instead, provide a tractable framework to address the mis-measurement of expenditure in the $\mathrm{CE}$, which is our main objective in this subsection. To the extent that respondent's errors are scaled by their level of expenditures, we shall see that the log-linear specification is particularly well suited to deal with such measurement error.

We then assume that the first-order expansion in true expenditure satisfies:

$$
\ln \left(e_{j, t}^{h}\right)^{*}-\ln \bar{e}_{j, t}^{*}=\alpha_{j, t}^{*}+\beta_{j} \ln e_{t}^{*}+\boldsymbol{\Gamma}_{j} \mathbf{Z}^{h}+\xi_{j, t}^{h},
$$

where $\mathbf{Z}^{h}$ is a vector of demographic dummies based on age range (25-37, 38$50,51-64)$, number of earners $(<2,2+)$, and household size $(\leq 2,3-4,5+)^{23}$. We allow the coefficient vector on demographics $\boldsymbol{\Gamma}_{j}$ to vary across goods. ${ }^{24}$ The variable $\alpha_{j, t}^{*}$ reflects an approximation of the expansion point of average total expenditure. The error term $\xi_{j, t}^{h}$ represents idiosyncratic relative taste shocks as well as the second-order error from the log-linear approximation, which we assume are independent of total expenditure and independent of expenditure elasticities $\beta_{j}$. The expenditure elasticity, $\beta_{j}$, does not have a time subscript, refecting the assumption that the expenditure elasticity for each good is stable over time ${ }^{25}$.

It is worth mentioning that relative prices do not appear explicitly in (6), although its specification accommodates changes in demand over time that are driven by shifts in relative prices. Since this subsection is aimed at estimating consumption inequality and not price effects, the first-order effect of changes in prices (the cumulation of own price effects and the effects due to cross-price elasticities) on demand for good $j$ at time $t$ are captured by the good-time intercept $\alpha_{j, t}^{*}-$ good-time specific effects.

Equation (6) can be rewritten in terms of observables:

\footnotetext{
${ }^{22}$ Namely, a log change in total expenditure will predict proportional changes in individual goods that do not necessarily add up to the assumed change in the total.

${ }^{23}$ We use the same variables in $\mathbf{Z}^{h}$ as in Aguiar and Bils (2015).

${ }^{24}$ Aguiar and Bils (2015) have explored an extension in which demographic taste-shifters are allowed to vary by income as well as good and the results are nearly the same.

${ }^{25}$ See the robustness section of Aguiar and Bils (2015) in which they explore in detail the validity of the stability of $\beta_{j}$.
} 


$$
\ln e_{j, t}^{h}-\ln \bar{e}_{j, t}=\alpha_{j, t}+\beta_{j} \ln e_{t}^{h}+\Gamma_{j} \mathbf{Z}^{h}+u_{j, t}^{h},
$$

where $u_{j, t}^{h}=\phi_{q_{k}, t}+v_{j, t}^{h}+\xi_{j, t}^{h}$. That is, the residual term includes income-specific systematic measurement error, the residual good-household specific measurement error and the idiosyncratic taste shocks. Note that by including the mean observed per capita expenditure on the left-hand side, the good-time specific measurement error, $\psi_{j, t}$, is differenced out and $\alpha_{j, t}=\alpha_{j, t}^{*}+\beta_{j}\left(\ln \left(e_{t}^{h}\right)^{*}-\ln e_{t}^{h}\right)^{26}$.

Before introducing the second-stage estimation, it is worth mentioning two issues. First, we estimate expenditure elasticities $\beta_{j}$ using the pooled base period 1980-1982 in the CE survey. These three waves represent the beginning of our sample. Aguiar and Bils (2011) discuss in detail second-stage estimates and it turns out that they are relatively stable with respect to the first-stage time period ${ }^{27}$. Thus, considering the stability of expenditure elasticities, we choose the beginning of our sample as the basis for estimating expenditure elasticities so that we benefit as much as possible from the size of the sample period considering that we have to avoid using the same sample in the first and second stages of the consumption inequality estimation to prevent correlated sampling error arising from our generated regressors.

Second, mis-measurement of individual goods is cumulated into total expenditure, inducing correlation between the measurement error captured in the residual and observed total expenditure in a demand system like (7). We report results using standard instruments to total per capita expenditure. We focus on the authors' baseline instruments to total per capita expenditure: dummies for the household's income quintile as well as the continuous variable $\log$ after-tax income. It exploits the fact that total expenditure reflects permanent income and will thus be correlated with current income. ${ }^{28}$ Note that although Aguiar and Bils (2015) show that expenditure elasticities are rather stable over time, still the pooled base period 1980-1982 CE may contain systematic mis-measurement that will lead to biased estimates of the expenditure

${ }^{26}$ We follow Aguiar and Bils (2015) in replacing $\ln e_{j, t}^{h}-\ln \bar{e}_{j, t}$ with the percentage deviation from average per capita expenditure on that good in that year: $\tilde{e}_{j, t}^{h} \equiv \frac{e_{j, t}^{h}-\bar{e}_{j, t}}{\bar{e}_{j, t}}$ since there are cases in which household expenditure on a particular good may be zero, which makes the log specification inappropriate. These authors have verified that the analysis does not depend on whether we use log total expenditure as the independent variable or the percent deviation from that year's average. These are equivalent representations in a first-order expansion around average expenditure. However, one has to keep in mind that households with large deviations may infuence the estimation in one or the other specification.

${ }^{27}$ These authors compare expenditure elasticities basically in the beggining (1980-1982), the mid-point (1994-1996) - baseline - and the end (2008-2010) of their sample and show they are rather stable.

${ }^{28}$ Aguiar and Bils (2011) also report a second approach to instrument, but they show that the two approaches yield nearly identical results. 
elasticities that may understate or overstate consumption inequality. For this reason, our ultimate estimates of inequality must be interpreted as conditional on the level of inequality observed in the first-stage surveys.

The second stage of the estimation recovers an estimate of how consumption inequality evolved by inverting demand system (6). First, expenditure is adjusted for demographics:

$$
\hat{e}_{j, t}^{h} \equiv \tilde{e}_{j, t}^{h}-\hat{\boldsymbol{\Gamma}}_{j} \mathbf{Z}^{h}
$$

where $\tilde{e}_{j, t}^{h} \equiv \frac{e_{j, t}^{h}-\bar{e}_{j, t}}{\bar{e}_{j, t}}$ and $\hat{\boldsymbol{\Gamma}}_{j}$ is the estimate of $\boldsymbol{\Gamma}_{j}$ from (6). Using (7) and by adding and subtracting $\beta_{j} \ln e_{q_{k}, t}^{*}$ in the right-hand side, we have:

$$
\hat{e}_{j, t}^{h}=\alpha_{j, t}+\phi_{q_{k}, t}+\beta_{j} \ln e_{q_{k}, t}^{*}+\varepsilon_{j, t}^{h},
$$

where $\varepsilon_{j, t}^{h}=\beta_{j}\left(\ln \left(e_{t}^{h}\right)^{*}-\ln e_{q_{k}, t}^{*}\right)+\xi_{j, t}^{h}+v_{j, t}^{h} 29$ and $\ln e_{q_{k}, t}^{*}$ is the average log per capita expenditure of income quintile $q_{k}$ at time $t$.

The empirical implementation of (8) follows: $\alpha_{j, t}$ are the coefficients of a vector of good-time dummies, $\phi_{q, t}$ are the coefficients of a vector of income-time dummies, $D_{q_{k}, t}$, and $\ln e_{q_{k}, t}^{*}$ are the coefficients on the interaction of incometime dummies and the first-stage estimates, $\hat{\beta}_{j}$. We estimate expenditure relative to the lowest income quintile $\left(q_{1}\right)$, which implies a consistent estimate of consumption inequality: $\delta_{q_{k}, t} \equiv \ln e_{q_{k}, t}^{*}-\ln e_{q_{1}, t}^{*}$ (normalized quintile expenditure) - our primary measure of consumption inequality. We regress $\hat{e}_{j, t}^{h}$ on these vectors of dummies and interactions for each year in the relevant sample period (1983-2010). Since the second stage uses a generated regressor, the first-stage estimates $\hat{\beta}_{j}$, we adjust the standard errors by bootstrapping. ${ }^{30}$

The key identification assumption is that $\varepsilon_{j, t}^{h}$ must be independent of $\beta_{j} \times D_{q_{k}, t}$. More exactly, we require that the good-time-household mismeasurement, $v_{j, t}^{h}$, be orthogonal to the expenditure elasticity, $\beta_{j}$, conditional on income quintile so that we can obtain a consistent estimate of $\ln e_{q_{k}, t}^{*}$, up to a normalization, by least squares ${ }^{31}$. Intuitively consistent with nonhomothetic preferences, identification arises from the fact that if the total expenditure of quintile $q_{k \mid k>1}$ increases relative to that of quintile $q_{1}$, that increase will fall disproportionately on luxuries.

\footnotetext{
${ }^{29}$ We suppress in the notation an estimation error related to $\hat{\boldsymbol{\Gamma}}_{j}$ in the residual term.

${ }^{30}$ We draw 100 replications with replacement from the micro data for all years and reestimate both stages.

${ }^{31}$ As Aguiar and Bils pointed out, the presence of $\beta_{j}\left(\ln \left(e_{t}^{h}\right)^{*}-\ln e_{q_{k}, t}^{*}\right)$ in $\varepsilon_{j, t}^{h}$ is not an issue, as this will be orthogonal to our regressor by definition: $E\left[\beta_{j} D_{q_{k}, t} \times \beta_{j}\left(\ln \left(e_{t}^{h}\right)^{*}-\ln e_{q_{k}, t}^{*}\right) \mid \beta_{j}, D_{q, t}\right]=\beta_{j}^{2} D_{q_{k}, t}\left[E\left(\ln \left(e_{t}^{h}\right)^{*}-\ln e_{q_{k}, t}^{*} \mid D_{q_{k}, t}\right)\right]=$
} 
Note that implementing specification (8) exploits the relative expenditure of higher income quintiles households and the bottom quintile households also exploring variation over all goods. This double differencing corrects for measurement error that can vary over time by consumption category and income. Identification then relies on the linear demand system (stability of expenditure elasticities) and nonhomotheticity of preferences, which in turn means that an increase in total expenditure inequality, conditional on income elasticities, implies a relative shift in expenditure toward luxuries (vis-a-vis necessities) for the richest households.

Estimation of equation (8) is by weighted least squares (WLS) for each year, where the estimation weights reflect average shares of each good in NIPABEA data over the sample period. ${ }^{32}$ We also report estimations by ordinary least squares (OLS). However, one has to keep in mind that OLS weights all goods equally in the second stage, so that goods with small shares may be driving the results. Thus, we keep the WLS as our baseline estimation method for the computation of the quintiles' expenditure weights.

Interestingly, we can use the measure of consumption inequality $\hat{\delta}_{q_{k}, t}$ estimated in the empirical implementation of equation (8) for each $t$ in the sample $(t=1983-2010)$ to construct the corrected weights of income quintiles:

$$
\hat{\omega}_{q_{k}, t} \equiv \frac{\exp \left\{\hat{\delta}_{q_{k}, t}\right\}}{1+\sum_{k=2}^{5} \exp \left\{\hat{\delta}_{q_{k}, t}\right\}},
$$

where we normalize $e_{q_{1}, t}^{*}=1$ for all $t$, such that $\hat{\omega}_{q_{1}, t} \equiv \frac{1}{1+\sum_{k=2}^{5} \exp \left\{\hat{\delta}_{q_{k}, t}\right\}}$.

\subsubsection{3 \\ Estimation Results}

We now present the corrected weights of income quintiles in total expenditure by applying Aguiar and Bils (2011) 's methodology. As we mentioned in the previous subsection, we estimate first-stage expenditure elasticities $\beta_{j}$ in equation (7) using the pooled base period 1980-1982 CE survey, representing the beginning of our sample. See Table A.2 in Appendix A for the first-stage estimates. ${ }^{33}$

\footnotetext{
${ }^{32} \mathrm{We}$ calculate the share of each good out of total expenditure consumption for each year, then average the shares over the sample period 1980-2010 and use these shares to weight the goods in the second stage regression. For more details in the construction of the weights see Aguiar and Bils (2011).

${ }^{33}$ Remind that the estimates are reported by using dummies for the household's income quintile as well as the continuous variable log after-tax income as intruments to total per capita expenditure. It also includes the average share of each good out of total per capita expenditure and standard errors, which suggest the elasticity estimates have a reasonable degree of precision and are consistent with Aguiar and Bils (2011)'s results using the 19941996 CE survey.
} 


\begin{tabular}{lcc|cc}
\hline & $\begin{array}{c}\text { OLS } \\
\text { Consumption } \\
\text { expenditure }\end{array}$ & $\begin{array}{c}\text { WLS } \\
\text { Consumption } \\
\text { expenditure }\end{array}$ & $\begin{array}{c}\text { Before-tax } \\
\text { income }\end{array}$ & $\begin{array}{c}\text { After-tax } \\
\text { income }\end{array}$ \\
\hline$\delta_{q_{2},(08-10)}-\delta_{q_{2},(83-85)}$ & $\begin{array}{c}0.11^{* *} \\
(0.05)\end{array}$ & $\begin{array}{c}0.05 \\
(0.05)\end{array}$ & 0.03 & 0.07 \\
$\delta_{q_{3},(08-10)}-\delta_{q_{3},(83-85)}$ & $\begin{array}{c}0.09 \\
(0.06)\end{array}$ & $\begin{array}{c}0.01 \\
(0.05)\end{array}$ & 0.05 & 0.09 \\
$\delta_{q_{4},(08-10)}-\delta_{q_{4},(83-85)}$ & $\begin{array}{c}0.19^{* * *} \\
(0.07)\end{array}$ & $\begin{array}{c}0.12^{* * *} \\
(0.05)\end{array}$ & 0.14 & 0.17 \\
$\delta_{q_{5},(08-10)}-\delta_{q_{5},(83-85)}$ & $\begin{array}{c}0.34^{* * *} \\
(0.09)\end{array}$ & $\begin{array}{c}0.34 * * * \\
(0.07)\end{array}$ & 0.21 & 0.24 \\
\hline
\end{tabular}

Table 1.2: Trends in Consumption Inequality Based on Relative Expenditure Patterns and Trends in Income Inequality

Notes: This table reports the estimated change in consumption inequality for income quintiles versus the poorest income quintile obtained from the second-stage regressions in subsection 3.1.2. All sepecifications in columns 1-2 use the first-stage estimated expenditure elasticities reported in Table A.2 in appendix A. The estimated parameters, $\delta_{q_{k},(08-10)}-\delta_{q_{k},(83-85)}, q_{k} \in\left\{q_{2}, q_{3}, q_{4}, q_{5}\right\}$, represent the relative growth in total expenditure for higher-income quintiles households relative to the lowest-income households for the period 1983-1985/2008-2010. See the specification in equation (8) and discussion in the text for full details. The first column implements the second stage by OLS while the second column implements weighted least squares, using the average shares for 1980-2010 as weights from NIPA-BEA. The standard errors are calculated using a bootstrap with 100 replications.

Table 2 reports the second-stage regression estimates of the log change in consumption inequality from (8). We show the change in consumption inequality of income quintiles relative to the bottom quintile $\left(q_{1}\right)$. Since there is substantial year-to-year movement, reflecting in large part sampling error, we report the change in inequality between the average of the first three years and the average of the last three years of the second-stage sample (1983-1985/20082010). This is the estimate of $\delta_{q_{k},(08-10)}-\delta_{q_{k},(83-85)}$. The first column reports the second-stage estimates using OLS on consumption expenditure data, while the second column reports the second-stage estimates using WLS. The last two columns exhibit again two measures of log change (before-tax and after-tax) income inequality reported in Table 1 for easy of comparison.

We see that the OLS estimates are in general larger than the WLS estimates, which likely reflects goods with small shares driving the results. In addition, consumption inequality is higher and more precisely estimated the richer the income quintile and it is especially driven by the top quintile, $q_{5}$. Finally and more important, we see that the overall figures on the second-stage estimates of consumption inequality are rather consistent to their counterpart of income inequality, unlike Table 1, which shows that the rise of consumption inequality based on reported consumption in the CE survey is considerably 
smaller than the increase of income inequality. It then suggests that the estimation methodology succeeded in correcting systematic measurement error in the CE Survey, which is key to assess consumption patterns across households. In particular, we note that in quintile $q_{5}$ the rise of consumption inequality expenditure is higher than income inequality: 0.34 by OLS and 0.31 by WLS vis-a-vis 0.21 and 0.24 , measured on both before-tax income and before-tax income, respectively. However, Table A.3 in Appendix A shows that the increase of income inequality is in line with the rise of nondurable consumption inequality. Figure A.1 also depicts the weights of income quintiles for nondurables and suggest that it is due to the fact that durable consumption is particularly important in quintile $q_{5}$.

Finally, Figure 1.2 depicts the corrected weights of the income quintiles implied by the consumption inequality measure $\hat{\delta}_{q_{k}, t}$, as defined by equation (9) for each year in the relevant sample period. We also report the weights of the income quintiles computed directly by reported expenditure in the CE survey for comparison. Note that the extensive margin of expenditure has increased considerably toward the top quintile - the quintile in which households have the largest expenditure shares (intensive margin of expenditure) of services and the lowest expenditure share of goods. We see in the upper Graphs A, B, and C for quintiles $q_{1}, q_{2}$ and $q_{3}$, respectively, that the reported extensive margins of expenditure of these quintiles understate the downward trend of the corrected extensive margins of expenditure. Conversely, Graph E shows that the increase of the reported extensive margin of expenditure of quintile $q_{5}$ understates the upward trend of its corrected extensive margin of expenditure. ${ }^{34}$

\footnotetext{
${ }^{34}$ See Table A.4 in Appendix A that reports the change in the income-specific measurement error for higher-income respondents relative to lowest-income respondents in equation (8) between 1983-1985 and 2008-2010, $\phi_{q_{k},(08-10)}-\phi_{q_{k},(83-85)}$, where, more precisely, $\phi_{q_{k}, t} \equiv$ $\phi_{q_{k}, t-} \phi_{q_{1}, t}$. We see that the change in relative mis-measurement is more precisely estimated and more negative in $q_{5}$, which suggests that the CE-BLS is increasingly missing expenditure by the high-income households (relative to low-income households).
} 

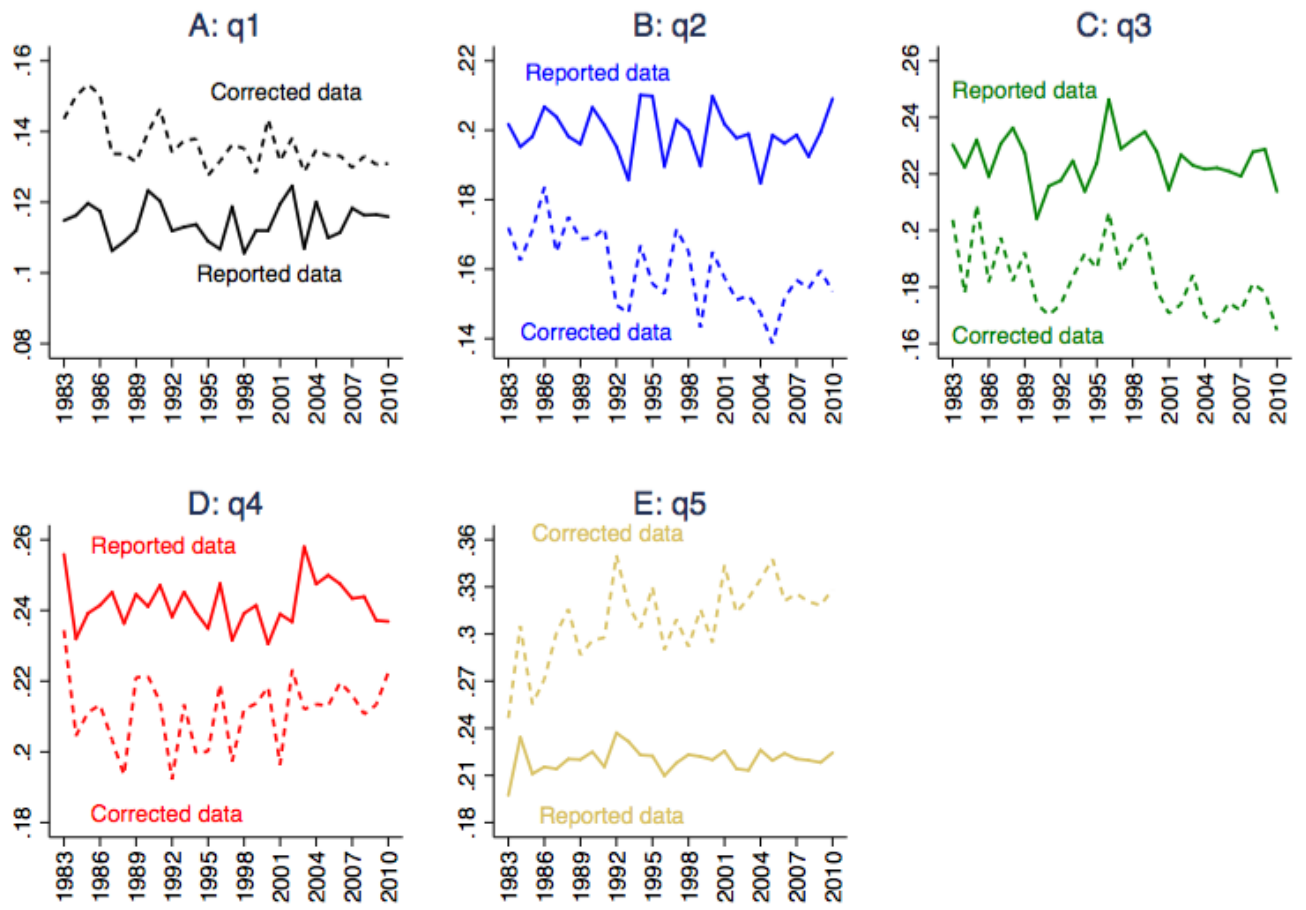

Solid line: reported data; Dashed line: corrected data

Figure 1.2: Trends of the Quintiles' Expenditure Weights in Total Expenditure Computed From Reported Data in the CE Survey and Constructed From the Second-stage Regressions (Corrected Data)

\footnotetext{
Notes: This figure depicts the weights of the average of each income quintile (quintile 1-quintile 5: $q_{1}-q_{5}$ ) with respect to total consumption expenditures from reported data in CE survey and obtained from the secondstage regressions in subsection 3.1.2. Quintiles' figures at a point in time add up to one. The weights obtained from from the second-stage regressions represent the ratio of the relative expenditure of each quintile to the sum of quintiles' relative expenditures. See the specification in equation (9) and discussion in the text for full details. Solid lines and Dashed lines denote, respectively, reported data (computed directly from reported data in $\mathrm{CE}$ survey) and corrected data (obtained from the second-stage regressions).
}

\subsection{2}

\section{Intensive Margin of Consumption Expenditure: quintiles' sectoral expen- diture shares}

\subsubsection{1}

\section{Data}

We use two sources of data for the estimation of the intensive margin of consumption expenditure: consumption expenditure data and prices data ${ }^{35}$. For consumption expenditure data we apply the same database from the CEBLS used to estimate the quintiles' weights in subsection 3.1.1. That is, our three-sector demand system (agriculture, manufacturing and services) is spec-

\footnotetext{
${ }^{35}$ Similarly to subsection 3.1.1, we report in Appendix A results using a nondurable version of the consumption sectors.
} 
ified over final-good consumption constructed by aggregating 22 consumption categories. For prices data we use the CPI-BLS (CPI-U - U.S. city average).

Although we are not completely unresctricted in terms of disaggregated consumption items - as stated above, the database is aggregated in 22 consumption categories $^{36}$ - these two surveys are coupled. There is a direct link between consumption items in the CE and CPI items since the former items compose the annual weights of the consumption goods in the basket comprised by the CPI. Therefore, price items are naturally assigned to each consumption category in most cases.

With respect to consumption expenditure data, as we mentioned, we use household per capita expenditure data since our model is specified in per capita terms. Our focus is on assessing differences in cross-sectional experiences of structural change, in particular over households grouped by income quintiles. For consistence with previous work, which use aggregate data, the definition of the sectors is done by aggregating over consumption categories in our database from CE-BLS to maximize the match with Herrendorf et al. (2009), subject to some consistency issues due to data source differences, by using the classification of total personal consumption expenditures in Table 2.4.5 - "Personal Consumption Expenditures by Type of Product" from NIPABEA. ${ }^{37}$ Note that for estimating the utility function parameters of equation (3) in a consistent way with the aggregate demand system in Herrendorf et al. (2009), we need to reproduce a similar data generator process in aggregate terms in our data from CE-BLS. That is, our definition of sectoral expenditure

${ }^{36}$ The allocation of consumption categories among sectors is limited by definitions in Aguiar and Bils (2011). For every category they constructed a nondurable measure of expenditure. Thus, we are able to disentangle categories among services and manufacturing sectors whenever a category comprises durables and services. In this case, the nondurable part is allocated in the services sector, as for example, the nondurable part of "funitures fixture" and the nondurable part of "vehicle purchase, leasing and insurance". However, for example in the category "all other transportation", which is composed basically by motor fuels and public transportation, we could not split it among services and manufacturing sectors since its regular measure equals its nondurable measure. Hence, we decide to allocate it in the manufacturing sector since public transportation accounts for $20 \%-30 \%$ of total expenditure in this category in average during the sample period, according to public available CE data by quintiles of income before taxes - current link: https://www.bls.gov/cex/csxcombined.htm.

${ }^{37}$ This replication is subject to smaller consistency issues due to differences in the level of aggregation. Furthermore, since CE-BLS data only cover household expenditure, we exclude purchases by nonprofit institutions serving households, purchases by U.S. government civilian and military personnel stationed abroad and do not include government consumption expenditure from NIPA-BEA data for the matching with CE-BLS data. Yet one has to keep in mind that fringe benefit payments made by an employer are accounted in the NIPA-BEA data, but are not reported in the CE-BLS data. Herrendorf et al. (2009) also use NIPABEA Table 7.1 for population data. All these consistency issues imply a level shift of the sectoral expenditure shares with respect to that in Herrendorf et al. (2009). Since we exclude expenditure categories that comprise the services sector, it implies the expenditure share of this sector is lower and those of agriculture and manufacturing are higher than the respective sectoral expenditure shares in Herrendorf et al. (2009). 
and relative prices should implie alike trajectories with respect to that using NIPA-BEA. Figures A.2-A.4 in Appendix A suggests a rather reasonable data source compatibility with respect to prices, aggregate quantities and associated aggregate expenditure shares. ${ }^{38}$

Regarding prices data, we assign each consumption category mentioned above to a CPI item. Then we set price indexes for consumption sectors at a point in time by averaging of the CPI items that compose each sector by the respective expenditure shares in NIPA-BEA (weights), following Aguiar and Bils (2011)'s matching between each expenditure groups in CE-BLS and itens of household expenditure in NIPA-BEA. Table 3 depicts the assignment of each consumption category and associated CPI itens to the three broad consumption sectors ${ }^{39}$.

We now turn to Figures 3-4, which show the evolution of price and consumption expenditure ${ }^{40}$ - the predetermined variables in our demand system. Looking at Figure 1.3, we see that the price of services has increased relative to agriculture and to manufacturing and the price of agriculture has increased relative to manufacturing ${ }^{41}$. Turning next to Figure 1.4, the quantity of manufacturing has grown the most, while the quantity of agriculture has grown the least ${ }^{42}$ in all income quintiles (graphs A-E) and consistently with aggregate data (graph F).

\footnotetext{
${ }^{38}$ We follow the cyclical expansion procedure in the online Appendix of Herredorf et al. (2013) to construct the sectoral prices and aggregate quantites.

${ }^{39}$ See Table A.5 in Appendix A for the assignment of each nondurable consumption category and associated CPI itens to the three broad consumption sectors

${ }^{40}$ Price and quantity indexes normalized in 1983 to 1.

${ }^{41}$ In special due to durable manufacturing goods. See nondurable price indexes in Figure A.3 in Appendix A.

${ }^{42}$ Nondurable manufacturing and services have grown similarly. See nondurable quantities indexes in Figure A.5 in Appendix A.
} 


\begin{tabular}{|c|c|c|}
\hline Sector & CE consumption category & CPI item \\
\hline Services & Housing & Shelter \\
\hline Agriculture & Food at home & Food at home \\
\hline Manufactured & All other tansportation & Transportation \\
\hline Manufactured & Vehicle purchasing & New vehicles \\
\hline Services & Utilities & Utilities \\
\hline Services & Food away from home & Food away from home \\
\hline Manufactured & $\begin{array}{l}\text { Appliances, phones, computers } \\
\text { with associated services }\end{array}$ & Durables \\
\hline Services & Health expenditures including insurance & Medical care \\
\hline Services & $\begin{array}{l}\text { Entertainment equipment and } \\
\text { subscription television }\end{array}$ & Personal care services \\
\hline Manufactured & Men's and women's clothing & Apparel \\
\hline Services & Vehicle leasing and insurance & Transportation services \\
\hline Services & Entertainment fees, admissions, reading & Admissions + recreational reading \\
\hline Services & Cash contributions (not for alimony/support) & Services \\
\hline Manufactured & Alcoholic beverages & Alcoholic beverages \\
\hline Manufactured & Shoes and other apparel & Apparel \\
\hline Manufactured & Furniture purchasing & Furnishing operations \\
\hline Manufactured & Tobacco, other smoking & Non-durables less food, beverages and appare \\
\hline Services & Education & Tuition, other school fees, and childcare \\
\hline Services & Personal care & Personal care products \\
\hline Services & Domestic services and childcare & Personal care services \\
\hline Manufactured & Children's clothing (up to age 15) & Apparel \\
\hline Services & Furniture fixtures & Personal care services \\
\hline
\end{tabular}

Table 1.3: Association of Broad Consumption Sectors, CE Consumption Categories and CPI Items

Notes: This table depicts the assignment of each consumption category in the CE-BLS and associated CPI itens (CPI-BLS) to the three broad consumption sectors that follows the sectors definitions in Herrendorf et al. (2009). For more details on definitions of each sector see Herrendorf et al. (2009). For details on sample construction see the data section and Aguiar and Bils (2011).

Figures 3-4 also suggest qualitative features of the utility specification that our estimation will use. For example, as the price of agriculture relative to manufacturing has increased, the quantity of agriculture relative to manufacturing has decreased, which is consistent with there being substitutability between agriculture and manufacturing in the aggregate and it is similarly valid for all income quintiles. However, our database suggests that aggregate data may suppress important cross-sectional differences of household consumption patterns. 


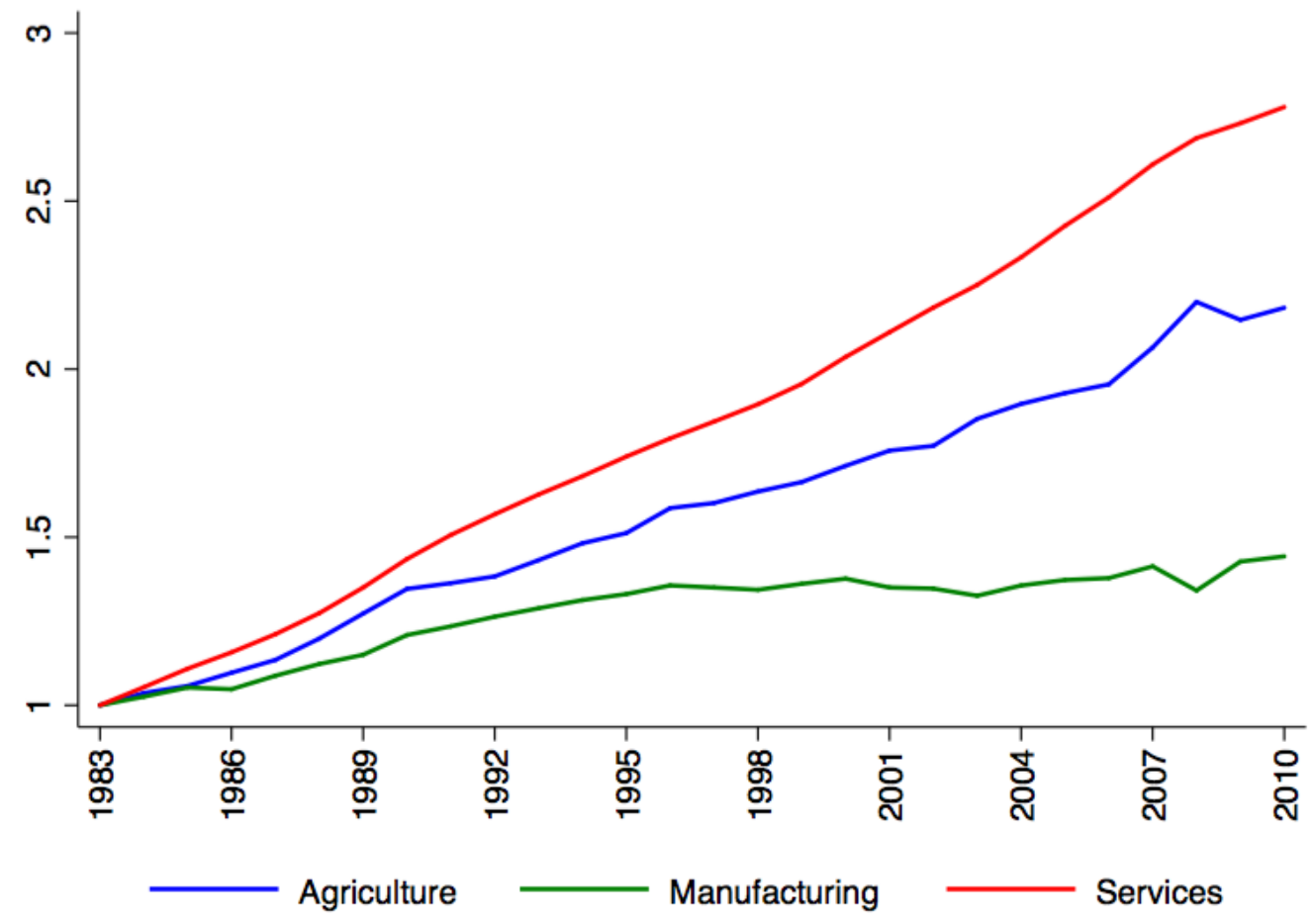

Figure 1.3: Sectoral Price Indices

Notes: This figure depicts the approximation of sectoral price indexes associated to consumption expenditure using data from CE-BLS for agriculture, manufacturing and services. Price indexes are constructed with CPIBLS data obtained by weighted-average (by the expenditure share of the equivalent consumption group of CE-BLS in NIPA-BEA) of the CPI itens attributed to each consumption group that compose agriculture, manufacturing and services. Data is normalized in 1983 to 1 . See the data sectionfor further details on definitions.

First, note that the price of services has increased relative to that of agriculture, while at the same time in aggregate data the quantity of services has also increased relative to that of agriculture, which is consistent with nonhomothetic utility specification - there is relative price and relative quantitiy moving in the same direction. However, while the quantities of services have increased relative to that of agriculture, the same conclusion arises both with decaying quantities of services and agriculture in quintiles $q_{1}-q_{3}$ (graphs A-C) and with rising quantities of services and agriculture in quintiles $q_{4}-q_{5} .{ }^{43}$ We discuss its implications in subsection 3.2 .3 on the estimation results of our demand system.

${ }^{43}$ See Figure A.5 in Appendix A for the sectoral quantity indexes by income quintiles in nondurable expenditure. 

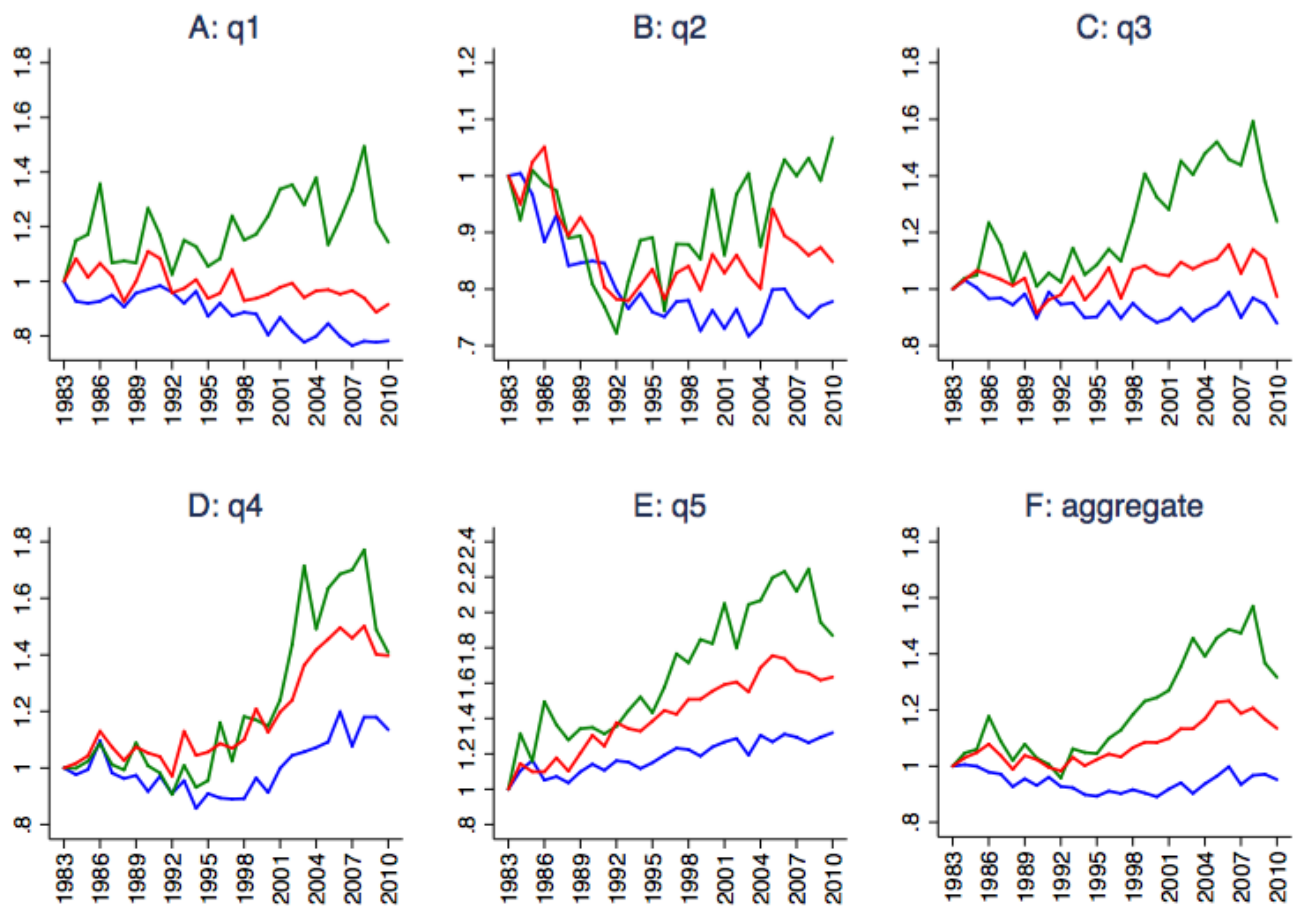

Figure 1.4: Sectoral Quantity Indexes by Income Quintiles

Notes: This figure depicts the sectoral quantity indexes considering consumption expenditure using data from CE-BLS for the agriculture, manufacturing and services sectors. Quantity indexes are obtained by deflating expenditure with the weighted-average (by the expenditure share of the equivalent consumption category of CE-BLS in NIPA-BEA) of the CPI itens attributed to each consumption category that compose the agriculture, manufacturing and services sectors. Data is normalized in 1983 to 1 . See the data subsection for further details on definitions.

The sectoral expenditure shares complete the data description - the dependent variables in the demand system. Figure 1.5 suggests that the standard (and asymptotic) pattern of structural change also shows up in the cross-section by income quintiles: the expenditure shares of agriculture (graph A) and manufacturing (graph B) are falling, while those of services (graph C) are increasing ${ }^{44}$. Similarly to Boppart (2014), we see that poorer households exhibit a larger expenditure share of agriculture as opposed to a smaller expenditure share of services. In manufacturing this income-ordered pattern does not show up, especially due to durable goods. As we mentioned, durable goods differences in expenditure across income quintiles do not necessarily align with differences in durable stocks and associated service flows and therefore it might obliterate cross-sectional differences of consumption patterns that we

${ }^{44}$ We report linear trends for easy of presentation. 
mean to address in the demand system ${ }^{45}$.

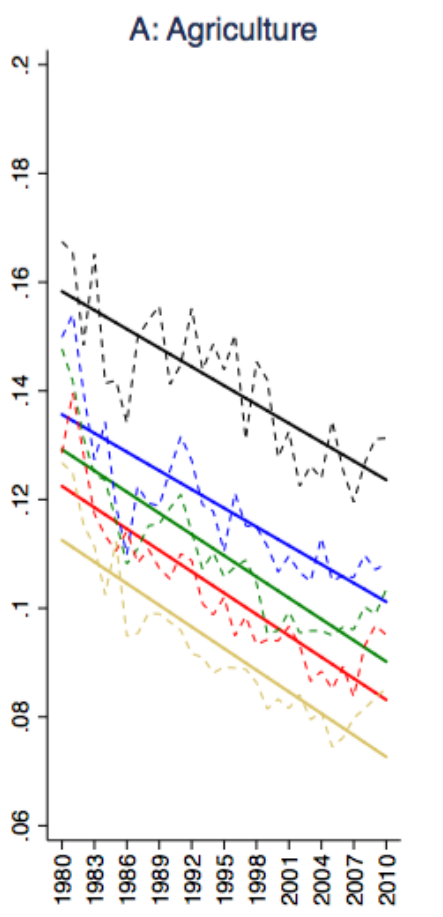

Solid line: linear trend; Dashed Line:data
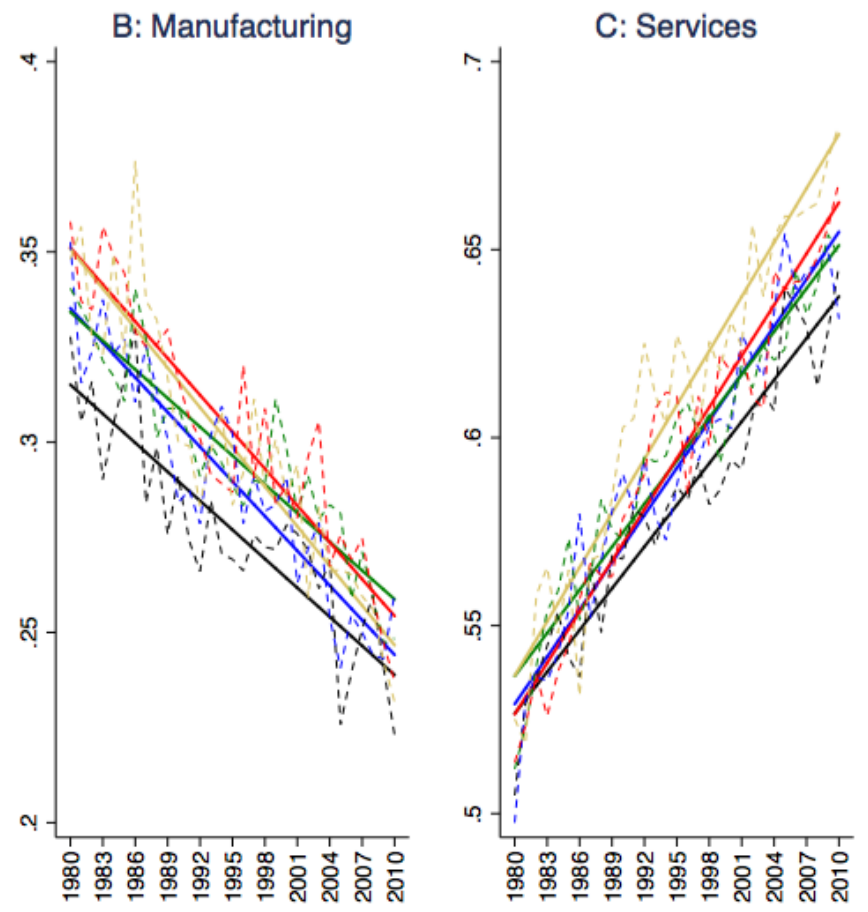

Black: q1; Blue: q2; Green: q3; Red: q4; Sand: q5

Figure 1.5: Trends in Sectoral Expenditure Shares by Income Quintiles

Notes: This figure depicts the consumption expenditure shares by quintiles of before-tax income respondents (quintile 1-quintile 5: $q_{1}-q_{5}$ ) in agriculture, manufacturing and services using CE-BLS data. Dashed lines and solid lines denote, respectively, data and linear trends. See the data section for further details on definitions.

\subsubsection{2}

\section{Estimation}

Now we estimate the parameters of the demand system (3). We closely follow previous studies in the literature in employing the iterated feasible generalized nonlinear least square to estimate the share equations both in the aggregate and by income quintiles. This is a standard way of estimating demand systems ( Deaton (1986); Herrendorf et al. (2009) ; Moro et al. (2017)).

Consider the expenditure share in each sector in equation (3) for each income quintile $q_{k}$. Given the set of predetermined variables,

$$
\mathbf{x}_{q_{k}, t} \equiv\left(p_{a, t}, p_{m, t}, p_{s, t}, e_{q_{k}, t}\right)
$$

${ }^{45}$ We also report results on nondurable expenditure in Figure A.6 in Appendix A. This income-expenditure pattern becomes clearer in nondurable manufacturing and then affects this pattern in the services sector, which shows up even sharper. In Figure A.7 in Appendix A, we add up the goods sectors (agriculture and manufacturing) so that one can see a clear opposite trend between goods and services and the expenditure shares ordered from the poorest quintile to the richest quintiles. 
and given the set of parameters

$$
\boldsymbol{\theta}_{q_{k}} \equiv\left(\bar{c}_{q_{k}, a}, \bar{c}_{q_{k}, s}, \varphi_{q_{k}, a}, \varphi_{q_{k}, m}, \varphi_{q_{k}, s}\right)
$$

we assume a stand-in household in each income quintile $q_{k}$ solves the intratemporal problem (1) - (2) for three shares, $\left\{\frac{p_{i, t} c_{q_{k}, i, t}}{e_{q_{k}, t}}\right\}$, for each sector $i$. Note we further assume that all households at a point in time face a common set of prices. We maintain this standard assumption, but acknowledge the caveat that prices may vary across households due to the ability to $\operatorname{search}^{46}$.

Since sectoral shares add up to one, the error covariance matrix is singular. Thus, we drop the demand for manufactured goods when we do the estimation and we are left with two nonlinear equations to be estimated:

$$
\begin{gathered}
s_{q_{k}, a, t} \equiv \frac{p_{a, t} c_{q_{k}, a, t}}{e_{q_{k}, t}}=f_{a}\left(\mathbf{x}_{q_{k}, t} ; \boldsymbol{\theta}_{q_{k}}\right)+\epsilon_{a} \\
s_{q_{k}, s, t} \equiv \frac{p_{s, t} c_{q_{k}, s, t}}{e_{q_{k}, t}}=f_{s}\left(\mathbf{x}_{q_{k}, t} ; \boldsymbol{\theta}_{q_{k}}\right)+\epsilon_{s}
\end{gathered}
$$

where $f_{a}$ and $f_{s}$ are, respectively, the expenditure share of agriculture and the expenditure shares of services as in equation (3); and $\epsilon_{a}, \epsilon_{s}$ are the error terms assumed to be uncorrelated with the predetermined variables ${ }^{47}$.

Note that the estimation results are not affected by which equation we drop due to the iteration over the parameters of the error covariance matrix. To deal with the issue that three out of our six parameters are constrained (i.e, $\varphi_{q_{k}, i} \geq 0$ and $\sum_{i=a, m, s} \varphi_{q_{k}, i}=1$ ), we transform the constrained parameters into unconstrained parameters as follows:

$\varphi_{q_{k}, a}=\frac{1}{1+\exp \left\{b_{1, q_{k}}\right\}+\exp \left\{b_{2, q_{k}}\right\}}, \varphi_{q_{k}, m}=\frac{\exp \left\{b_{1, q_{k}}\right\}}{1+\exp \left\{b_{1, q_{k}}\right\}+\exp \left\{b_{2, q_{k}}\right\}}, \varphi_{q_{k}, s}=\frac{\exp \left\{b_{2, q_{k}}\right\}}{1+\exp \left\{b_{1, q_{k}}\right\}+\exp \left\{b_{2, q_{k}}\right\}}$,

where $b_{1, q_{k}}, b_{2, q_{k}} \in(-\infty,+\infty)$.

After estimating the model in terms of the unconstrained parameters, $b_{1, q_{k}}, b_{2, q_{k}}$, and $\bar{c}_{q_{k}, a}, \bar{c}_{q_{k}, s}$, we transform these back to compute point estimates and standard errors for the original parameters, $\varphi_{q_{k}, a}, \varphi_{q_{k}, m}, \varphi_{q_{k}, s}$.

\footnotetext{
${ }^{46}$ See, for example, Aguiar and Hurst (2007).

${ }^{47}$ This demand system falls into the nonlinear seemingly unrelated regression framework and then in general equations are related through the covariance structure of the error terms. Considering that assumption over the error terms, iterating on the feasible generalized nonlinear least square estimator produces a sequence of parameter estimates that converges to the maximum likelihood estimate. See Greene (2003), chapter 14.9.3. and Deaton (1986) for further discussion related to the estimation of demand systems.
} 


\subsubsection{3 \\ Estimation Results}

Table 4 shows the results for the aggregate and for each income quintile using consumption expenditure from the CE-BLS survey and index prices from the CPI-BLS in the sample period, 1983-2010. We first note that, although BLS data is noisier than BEA data used by Herrendorf et al. (2009), it does not affect considerably the precision of the estimates. By comparing the results of the same model reported by these authors and the aggregate estimates of consumption expenditure in the sixth column of Table 4, we note that the estimates are similar. ${ }^{48}$

We also see that the aggregate estimates, which are consistent with those for a stand-in household with preferences of the same form, actually aggregate heterogeneous experiences of household sectoral demand across income quintiles. Even though our sample period, which starts in the 1980's, depicts a rich US and a considerable part of the structural change transition has already taken place in the aggregate, the estimate results reveal different consumption patterns across households grouped in income quintiles. We can see it through several ways. There are four points worth emphasizing here. First, there are opposite trends of both preference weights, $\varphi_{q_{k}, i}$, and nonhomotheticity terms, $\bar{c}_{q_{k}, j}$, between goods and services sectors across income quintiles. Overall, while $\varphi_{q_{k}, a}, \varphi_{q_{k}, m}$ and $\bar{c}_{q_{k}, a}$ decrease as income quintile rises, we see the opposite for $\varphi_{q_{k}, s}$ and $\bar{c}_{q_{k}, s}$.

${ }^{48}$ For example, see the second column of Table 1 in Herrendorf et al. (2009)). One has to keep in mind that, as mentioned in the section on dataset description, unlike these author's we do not consider for example government consumption in the services sector since we use CE-BLS data. Thus, one should expect, on the one hand, that it lowers the estimates of the services weight, $\varphi_{q_{k}, s}$, and the nonhomotheticity term o services, $\bar{c}_{q_{k}, s}$. On the other hand, whereas Herrendorf et al. (2009) cover all the postwar period (1947-2010), we consider a more recent subperiod (1983-2010), which should imply a rise of these estimates since it represents a richer economy. In the sixth column of Table 4, the estimate for $\bar{c}_{s}$ in the aggregate is statistically significant at 17 percent level. 


\begin{tabular}{|c|c|c|c|c|c|c|}
\hline & \multicolumn{6}{|c|}{ Consumption expenditure } \\
\hline & $\mathrm{q}_{1}$ & $\mathrm{q}_{2}$ & $\mathrm{q}_{3}$ & $\mathrm{q}_{4}$ & $\mathrm{q}_{5}$ & Aggregate \\
\hline $\bar{c}_{a}$ & $\begin{array}{c}-379.39 * * * \\
(76.18)\end{array}$ & $\begin{array}{c}-368.37 * * * * \\
(65.42)\end{array}$ & $\begin{array}{c}-720.74 * * * \\
(71.94)\end{array}$ & $\begin{array}{c}-277.00 * * * \\
(104.76)\end{array}$ & $\begin{array}{c}-306.60^{* * * *} \\
(75.85)\end{array}$ & $\begin{array}{c}-688.37 * * * \\
(66.88)\end{array}$ \\
\hline $\bar{c}_{s}$ & $\begin{array}{c}-1,808.21^{* * *} \\
(99.38)\end{array}$ & $\begin{array}{c}-2,375.19 * * * \\
(177.42)\end{array}$ & $\begin{array}{c}-1,978.30^{* * * *} \\
\quad(701.94)\end{array}$ & $\begin{array}{l}4,779.38^{*} \\
(2,648.89)\end{array}$ & $\begin{array}{c}13,574.92^{* *} \\
(6,664.07)\end{array}$ & $\begin{array}{c}1,137.25 \\
(3,115.08)\end{array}$ \\
\hline$\varphi_{a}$ & $\begin{array}{c}0.14^{\text {**** }} \\
(0.02)\end{array}$ & $\begin{array}{c}0.12^{* * * *} \\
(0.02)\end{array}$ & $\begin{array}{c}0.04 * * * \\
(0.01)\end{array}$ & $\begin{array}{c}0.04 * * * \\
(0.01)\end{array}$ & $\begin{array}{c}0.02 * * * \\
(0.005)\end{array}$ & $\begin{array}{c}0.03^{* * *} \\
(0.01)\end{array}$ \\
\hline$\varphi_{m}$ & $\begin{array}{c}0.53^{* * * *} \\
(0.03)\end{array}$ & $\begin{array}{c}0.50^{* * *} \\
(0.02)\end{array}$ & $\begin{array}{c}0.47^{* * *} \\
(0.08)\end{array}$ & $\begin{array}{c}0.19^{* * *} \\
(0.04)\end{array}$ & $\begin{array}{c}0.11^{* * * *} \\
(0.03)\end{array}$ & $\begin{array}{c}0.26^{* * *} \\
(0.12)\end{array}$ \\
\hline$\varphi_{s}$ & $\begin{array}{c}0.32^{\text {**** } *} \\
(0.03)\end{array}$ & $\begin{array}{c}0.38^{* * * *} \\
(0.03)\end{array}$ & $\begin{array}{c}0.49^{* * *} \\
(0.09)\end{array}$ & $\begin{array}{c}0.77^{* * * *} \\
(0.05)\end{array}$ & $\begin{array}{c}0.87^{* * * *} \\
(0.04)\end{array}$ & $\begin{array}{c}0.71 * * * \\
(0.13)\end{array}$ \\
\hline$\chi^{2}\left(\bar{c}_{a}=0, \bar{c}_{s}=0\right)$ & $349.32 * * *$ & $323.34^{* * * *}$ & $100.39 * * *$ & $28.03^{* * *}$ & $62.69 * * *$ & $116.09^{* * * *}$ \\
\hline$\chi^{2}\left(\theta_{q_{k}}=\theta_{A g g}\right)$ & $15,212.12 * * *$ & $2,722.31$ *** & $546.47 * * *$ & $813.09 * * *$ & $3,407.62 * * *$ & - \\
\hline$L R\left(\theta_{\text {Agg }}\right.$ nested in $\left.\left\{\theta_{q_{k}}\right\}_{k=1}^{5}\right)$ & - & - & - & - & - & $1,430.66 * * *$ \\
\hline$R M S E_{a}$ & 0.009 & 0.006 & 0.005 & 0.006 & 0.003 & 0.005 \\
\hline$R M S E_{m}$ & 0.012 & 0.018 & 0.021 & 0.027 & 0.023 & 0.027 \\
\hline$R M S E_{s}$ & 0.014 & 0.022 & 0.024 & 0.031 & 0.023 & 0.030 \\
\hline
\end{tabular}

Table 1.4: Sectoral Share Estimation Results for the Aggregate and Income Quintiles $\left(q_{1}-q_{5}\right)$

Notes: $\chi^{2}\left(\bar{c}_{a}=0, \bar{c}_{s}=0\right)$ is the Wald Test Statistics for the hypothesis that $\bar{c}_{a}$ and $\bar{c}_{s}$ are jointly zero. $\chi^{2}\left(\theta_{q_{k}}=\theta_{\text {agg }}\right)$ is the Wald Test Statistics for the hypothesis that each quintiles' coefficient estimates are jointly equal to the aggregate counterpart. $\operatorname{LR}\left(\theta_{\text {agg }}\right.$ nested in $\left.\left\{\theta_{q_{k}}\right\}_{k=1}^{5}\right)$ is the Likelihood-ratio test for the hypothesis that all coefficients of the aggregate model do not vary between income quintiles $q_{1}-q_{5} . R M S E_{j}$ is the root mean squared error for j-sector's share equation. Robust standard errors in parentheses. The calculation of standard errors of the constrained parameters is based on the delta method. *** Significant at the 1 percent level, $* *$ Significant at the 5 percent level and ${ }^{*}$ Significant at the 10 percent level.

Second, in general terms the signs of the two unrestricted nonhomothetic terms in the aggregate and in most quintiles have the pattern suggested by Kongsamut et al. (2001), that is, $\bar{c}_{a}<0$ and $\bar{c}_{s}>0$. Interestingly, $\bar{c}_{q_{1}, s}, \bar{c}_{q_{2}, s}$ and $\bar{c}_{q_{3}, s}$ are negative. It might seem tricky to interpret it in our environment, insofar as our model only consider market services data. To assess it in detail is not in the core of our study and it deserves further investigation. However, it is worth pointing out that it seems consistent with results in Moro et al. (2017), from which we follow our interpretation ${ }^{49}$. These authors include homeservices production data and split market services between traditional services

${ }^{49}$ Our result is also consistent with Mazzolari and Ragusa (2013) that use data from CE-BLS to show that the least-skilled workforce in the United States is disproportionally employed in the provision of time-intensive services that can be thought of as market substitutes for home services. Mazzolari and Ragusa (2013) also show evidence that the income elasticity of services that are substitutes for home services is substantially lower for households in the bottom portion of the income distribution, which suggest that the poorest households cannot easily substitute home services. 
(substitutable with home-services production) and modern services in a valueadded specification to estimate a Stone-Geary demand system that features parameters of nonhomotheticity associated to traditional market services and home-services production. Both parameters are estimated to be negative. Our final-good consumption specification prevent that we consider a variable of home-services production. Nevertheless, following these authors, $\bar{c}_{q_{k}, s}<0$ can be interpreted as a minimum requirement of home-services production and traditional market services consumption (for instance household maintenance and cleaning) that the household has to provide/consume before enjoying the rest of home-services and market services ${ }^{50}$.

Third, we reject the hypothesis that both nonhomotheticity terms are equal to zero, according to the Wald test statistics $\chi^{2}\left(\bar{c}_{a}=0, \bar{c}_{s}=0\right)$ reported in Table 4. Fourth, according to the other set of Wald test statistics, $\chi^{2}\left(\theta_{q_{k}}=\theta_{\text {agg }}\right)$, we reject that each quintiles' coefficient estimates are jointly equal to the aggregate counterpart. Similarly, we also reject the hypothesis that all coefficients of the aggregate model do not vary between disjointed subsets of the data, which is reported in the Likelihood-ratio test, $\operatorname{LR}\left(\theta_{\text {agg }}\right.$ nested in $\left.\left\{\theta_{q_{k}}\right\}_{k=1}^{5}\right)$ - a Chow-type test in the cross-section of households by income quintiles. ${ }^{51}$

Following the economic and statistical significance of the disaggregate estimates, we apply the empirical counterpart of the weighted-average aggregate expenditure shares defined in equation (4):

$$
\tilde{s}_{i, t} \equiv \sum_{k=1}^{5} \hat{\omega}_{q_{k}, t} \hat{s}_{q_{k}, i, t},
$$

where $\hat{\omega}_{q_{k}, t}$ is the estimated expenditure weights of income quintile $q_{k}$ defined in equation (9) and $\hat{s}_{q_{k}, i, t}$ is the predicted expenditure share of quintile $q_{k}$ of sector $i$ denoted by equation (3).

As we see by the reported root mean square errors in Table 4 , the fit of the model for most quintiles is even better than the fit of the model for aggregate data. Figures 6 and 7 illustrate it. We see that it specially applies to the fit of the manufacturing and services shares. These findings then imply a lower root mean square errors (RMSE) for the weighted-average aggregate expenditure shares, $\tilde{s}_{i, t}$, relative to that reported for the model applied on aggregate data - the RMSE of the weighted-average aggregate expenditure shares are $0.004,0.014,0.016$ vis-a-vis $0.005,0.027,0.030$ reported in Table 4 for

\footnotetext{
${ }^{50}$ Note that there is an hierarchical sense of the preferences in this interpretation.

${ }^{51}$ To compute the LR test we do not use robust standard errors in order to guarantee its validity and interpretability. However, it virtually does not affect the statistical significance of the estimates in general terms.
} 
model applied on aggregate data, respectively, in agriculture, manufacturing and services.
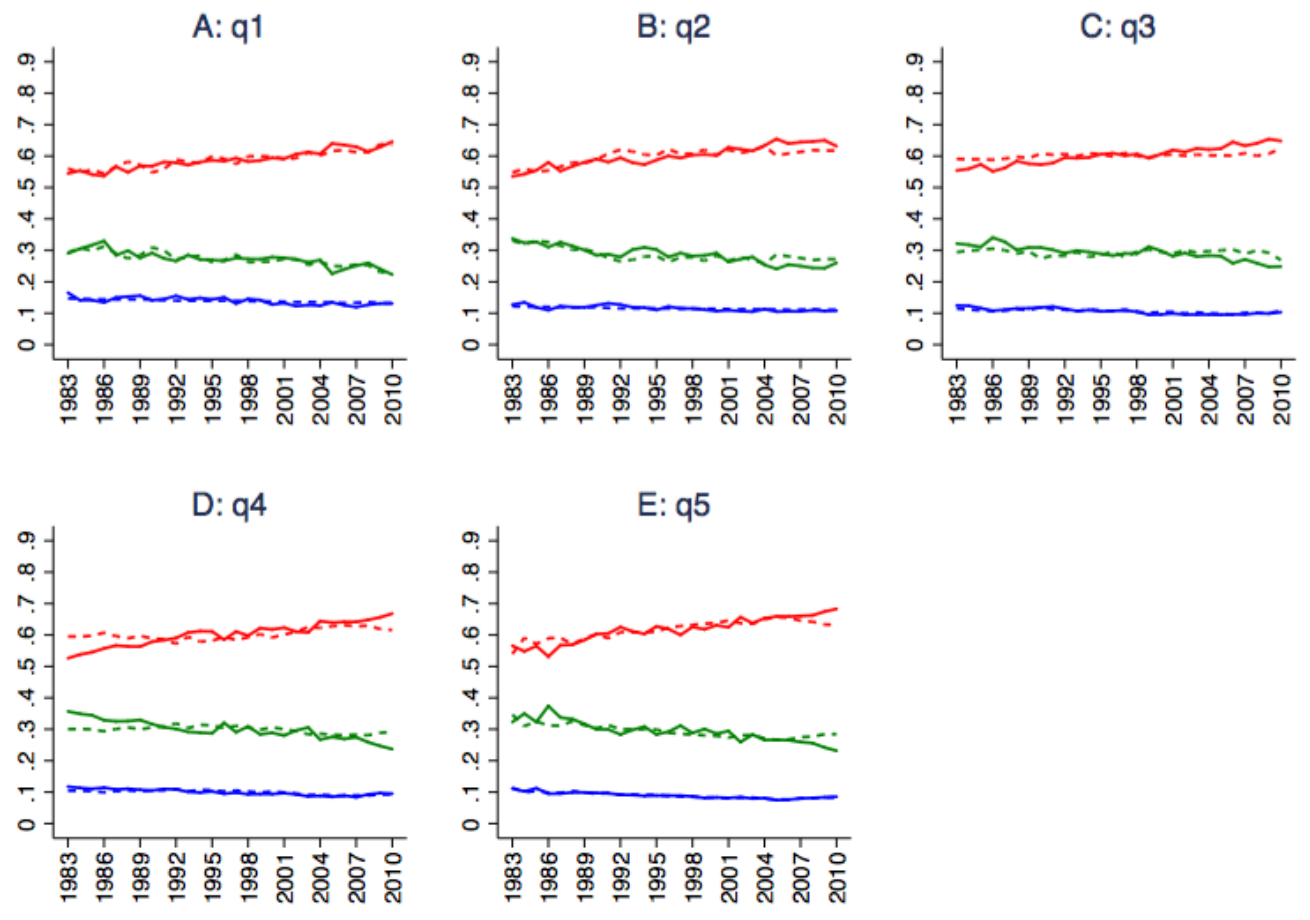

Solid line: data; Dashed line: model

Blue:Agriculture; Green: Manufactured; Red: services

Figure 1.6: Fit of the Model: Expenditure Shares by Income Quintiles

Notes: This figure depicts the fit of the model for sectoral expenditure shares by income quintiles (Graphs A-E, respectively, for quintile 1-quintile 5: $q_{1}-q_{5}$ ) in agriculture (blue), manufacturing (green) and services (red) as denoted by equation (3) using CE-BLS data for consumption expenditure. Solid lines and dashed lines denote, respectively, data, model prediction. See the data subsection for further details on definitions and the estimation subsection for the estimation procedure. 


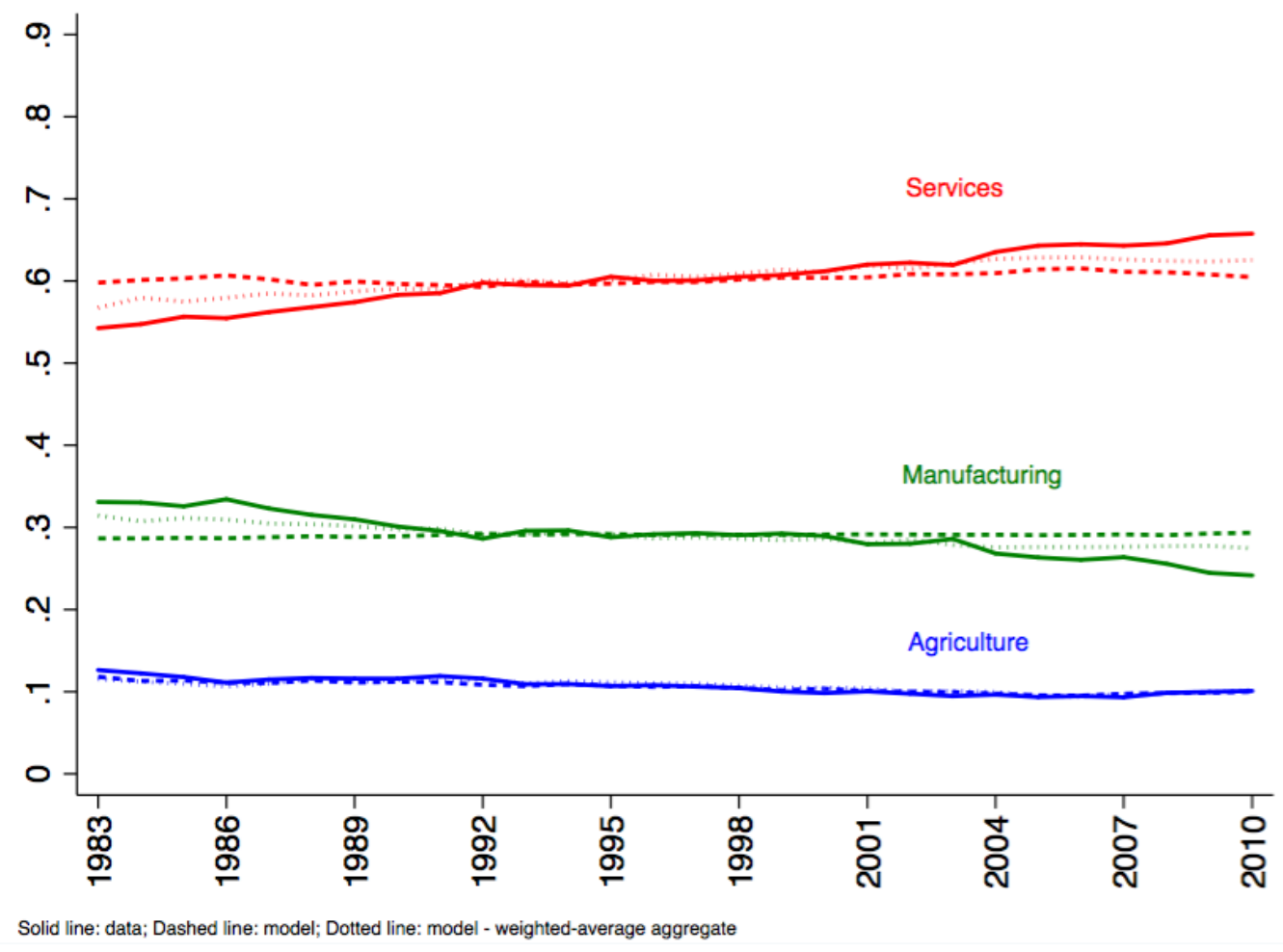

Figure 1.7: Fit of the Model: Expenditure Shares on Aggregate Data and Weighted-average Aggregate Expenditure Shares

Notes: This figure depicts the fit of the model for expenditure shares on aggregate data and the weightedaverage (by expenditure weights of quintile 1-quintile 5: $q_{1}-q_{5}$ ) aggregate expenditure shares in agriculture (blue), manufacturing (green) and services (red) using CE-BLS data for consumption expenditure. Solid lines, dashed lines and dotted lines denote, respectively, data, model prediction on aggregate data and model prediction for the weighted-average aggregate expenditure shares. See the data subsection for further details on definitions and the estimation subsection for the estimation procedure.

Finally, note that aggregate expenditure data broadly support our StoneGeary specification that follows Kongsamut et al. (1997) consistently with cross-sectional expenditure data. These authors impose the condition $p_{a, t} \bar{c}_{q_{k}, a}+$ $p_{s, t} \bar{c}_{q_{k}, s}=0$ for the existence of a generalized balanced growth. However, following Herrendorf et al. (2009), this condition is not consistent with the final consumption expenditure data. Figure 1.3 shows that $p_{s, t} p_{a, t}$ has been steadily increasing while $\bar{c}_{q_{k}, a}$ and $\bar{c}_{q_{k}, s}$ are constants. However, it does not seem problematic. Since the stylized fact states that balanced growth is a good approximate description of the data, there is no incompatibility. For example, simulations results for specifications that depart from the conditions required for exact balanced growth in Kongsamut et al. (1997) are not quantitatively significant in the sense that the resulting time series are still very close to satisfying balanced growth (See also Gollin et al. (2007) for similar calculations). 


\section{4}

\section{Income Effects, Consumption Inequality and Structural Change}

Following the implementation of the weighted-avarage aggregate sectoral expenditure shares, we are now able to assess whether there is a connection between consumption inequality and structural change. If so, particularly with respect to the intensive margin of consumption expenditure, it first passes through provide some perspective on the relative importance of changes in income vis-a-vis the importance of changes of relative prices in accounting for the observed changes in the sectoral shares of consumption expenditures. To explore this further, we stress below the relevance of income effects of changes in the sectoral shares across households grouped by income quintiles as an underlying mechanism through which consumption inequality correlates with structural change.

\subsection{1}

\section{Relevance of Income Effects of the Structural Change}

Before assessing the relative importance of income and price effects of changes in the sectoral shares of consumption expenditures, a first step, that is associated to the heterogeneous income effects of the households demands that we further explore below, is to present the quintiles' expenditure (income) elasticities. The first order conditions from equations (1) - (2) and the estimation of our demand system for households grouped by income quintiles in subsection 3.2.3 gives rise to the following empirical expenditure elasticities:

$$
\eta_{q_{k}, i, t}^{e}=\left(1+\frac{\hat{\bar{c}}_{q_{k}, i}}{c_{q_{k}, i, t}}\right)\left(\frac{e_{q_{k}, t}}{e_{q_{k}, t}+\sum_{g=a, m, s} p_{g, t} \hat{\bar{c}}_{q_{k}, g}}\right),
$$

where $\eta_{q_{k}, i, t}^{e}$ is the expenditure elasticity of quintile $q_{k}$ of sector $i$, such that the superscript $e$ denotes expenditure ${ }^{52} ; \hat{\bar{c}}_{q_{k}, i}$ is the estimated nonhomotheticity term of quintile $q_{k}$ of sector $i$ and $c_{q_{k}, i, t}$ and $e_{q_{k}, t}$ are, respectively, the per capita average consumption quantities of quintile $q_{k}$ of sector $i$ and the per capita average consumption expenditure of quintile $q_{k} \cdot{ }^{53}$

Figure 1.8 illustrates the expenditure elasticities by sectors across income quintiles. We see in Graphs A-C for the sectors that there are two different clusters of quintiles' elasticities. To this respect, we consider the uncertainty associated to the estimation of $\bar{c}_{q_{k}, j}$. We plot point estimates and confidence bands for the quintiles' elasticities at 90 percent level in the Graphs. ${ }^{54}$ The

\footnotetext{
${ }^{52}$ Due to constancy of $\bar{c}_{q_{k}, i}$, these elasticities converge to unity at the same rate as the rate of growth of $c_{q_{k}, i, t}: e_{q_{k}, t} \rightarrow+\infty \Rightarrow \eta_{q_{k}, i, t}^{e} \rightarrow 1$. Therefore, nonhomotheticity is a short-run feature of Stone-Geary preferences.

${ }^{53}$ Similarly to equation $(3), g=\{a, m, s\}$ here with a slight abuse of terminology.

${ }^{54}$ The calculation of standard errors is based on the delta method.
} 
confidence bands depict the contours of a heterogeneity of quintiles' elasticities through clusters of income quintiles. However, we cannot statistically differentiate the expenditure elasticities of the income quintiles within each cluster of quintiles' elasticities.
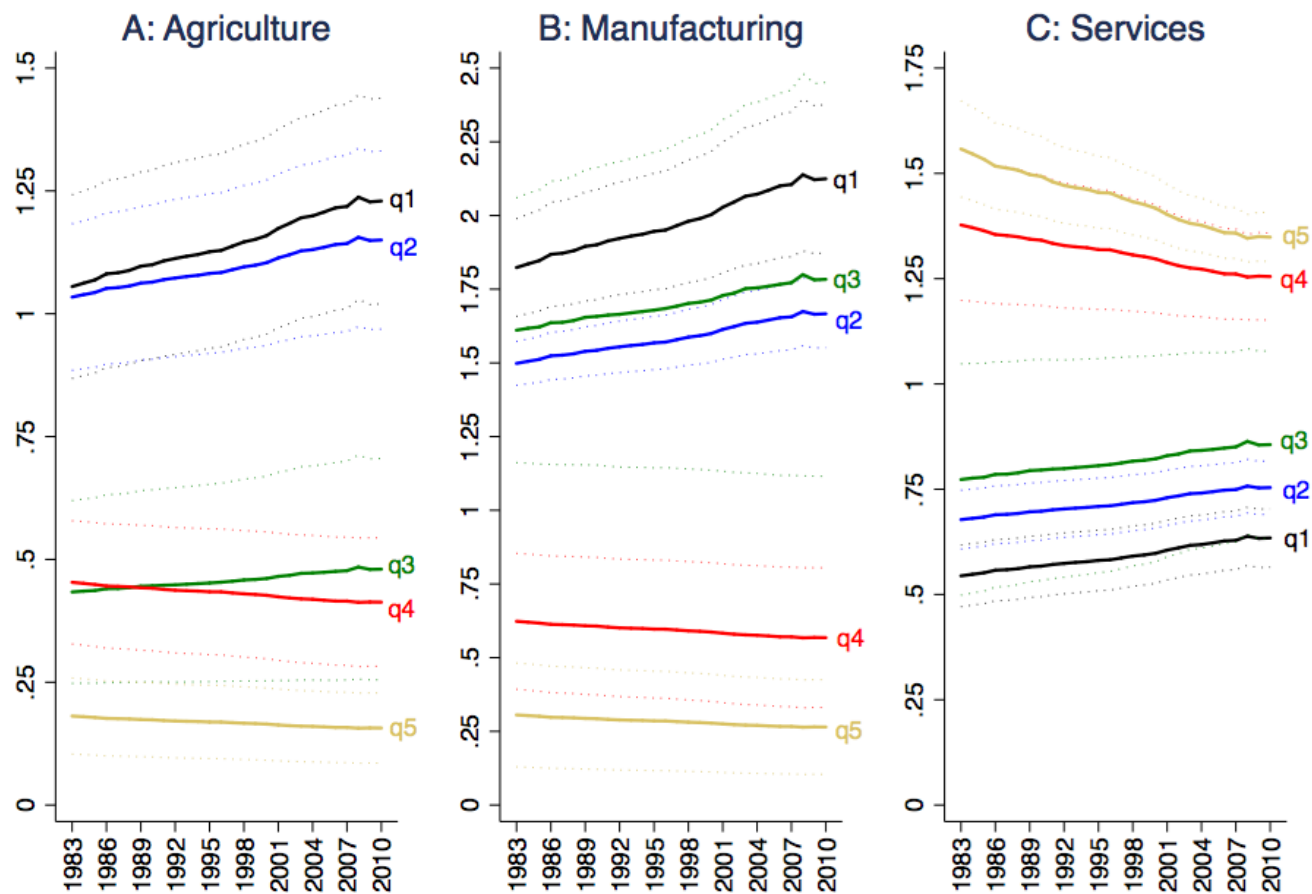

Solid lines: point estimates; Dotted lines: confidence bands at 90 percent

Figure 1.8: Trends in Sectoral Expenditure Elasticities by Income Quintiles

Notes: This figure depicts the sectoral expenditure elasticities by income quintiles (quintile 1-quintile 5: $q_{1}-q_{5}$ ) in agriculture (Graph A), manufacturing (Graph B) and services (Graph C) using CE-BLS data. Solid lines and dotted lines denote, respectively, point estimates and confidence bands at 90 percent based on standard errors calculated by the delta method. See equation (11) for the formal definition of the elasticities.

First, note in Graph A that goods from agriculture (food) are necessities ${ }^{55}$ for households income quintiles $q_{3}-q_{5}$ and according to the point estimates they are luxuries for quintiles $q_{1}-q_{2}$, although we cannot reject the hypothesis that food is a necessity for all income quintiles. Second, we see in Graph B that manufacturing goods are luxuries for the cluster composed by the poorest quintiles $q_{1}-q_{3}$ and necessities for the cluster composed by the richest quintiles $q_{4}-q_{5}$. In Graph $\mathrm{C}$ of the services sector, we see an opposite picture. Remind the estimates of the nonhomotheticity term of services reported in Table 4. More relevant than any interpretation of the negative estimates for quintiles $q_{1}-q_{3}$, it ultimately indicates what equation (11) describes: the expenditure of services for these quintiles are less elastic than those of quintiles $q_{4}-q_{5}$. Interestingly,

${ }^{55} \mathrm{~A}$ sectoral commodity good is a necessity for quintile $q_{k}$ if $\eta_{q_{k}, i, t}^{e} \leq 1$. Conversely, a sectoral commodity good is a luxury for quintile $q_{k}$ if $\eta_{q_{k}, i, t}^{e}>1$. 
this cross-sectional pattern of household consumption is consistent with US secular aggregate data that describes an asymptotic fall of the agriculture expenditure share, a hump-shaped manufacturing expenditure share and the services expenditure share rising assymptotically (see, for example, Herrendorf et al. (2013), p. 873).

Now we provide a perspective on the relative importance of changes in income and relative prices in accounting for the observed changes in the sectoral shares of aggregate consumption expenditure. We follow Herrendorf et al. (2009) in defining the fit of the expenditure shares implied by the income effect and the price effect. Unlike these authors, we do it through the weighted-average aggregate expenditure shares, our key measure of structural change, instead of assessing the fit of the expenditure shares implied by the income effect and the price effect applied on aggregate data. That is, these effects are special cases that arises from equation (10). It means the fit of the weighted-average aggregate expenditure shares implied by the income effect is expressed in terms of the parameters estimated in each income quintile according to equation (3) and reported in Table 4 under the counterfactual in which quintiles' expenditure change as dictated by the data but relative prices are held constant at their $t_{0}$ values $\left(t_{0}=1983\right)$ :

$$
\tilde{s}_{i, t}^{e} \equiv \sum_{k=1}^{5} \hat{\omega}_{q_{k}, t} \hat{s}_{q_{k}, i, t}^{e},
$$

where $\hat{s}_{q_{k}, i, t}^{e}=\hat{\varphi}_{q_{k}, i}\left(1+\sum_{g=a, m, s} \frac{p_{g, t_{0}} \hat{\bar{c}}_{q_{k}, g}}{e_{q_{k}, t}}\right)-\frac{p_{i, t_{0}} \hat{\bar{c}}_{q_{k}, i}}{e_{q_{k}, t}}$ and the superscript $e$ again denotes the income (expenditure) effect.

The fit of the weighted-average aggregate expenditure shares implied by the price effect, in turn, is expressed in terms of the parameters estimated in each income quintile under the counterfactual in which prices change as dictated by the data but quintiles' expenditure are held constant at their $t_{0}$ values:

$$
\tilde{s}_{i, t}^{p} \equiv \sum_{k=1}^{5} \hat{\omega}_{q_{k}, t} \hat{s}_{q_{k}, i, t}^{p},
$$

where $\hat{s}_{q_{k}, i, t}^{p}=\hat{\varphi}_{q_{k}, i}\left(1+\sum_{g=a, m, s} \frac{p_{g, t} \hat{\bar{c}}_{q_{k}, g}}{e_{q_{k}, t_{0}}}\right)-\frac{p_{i, t} \hat{\bar{c}}_{q_{k}, i}}{e_{q_{k}, t_{0}}}$ and the superscript $p$ denotes the price effect. 

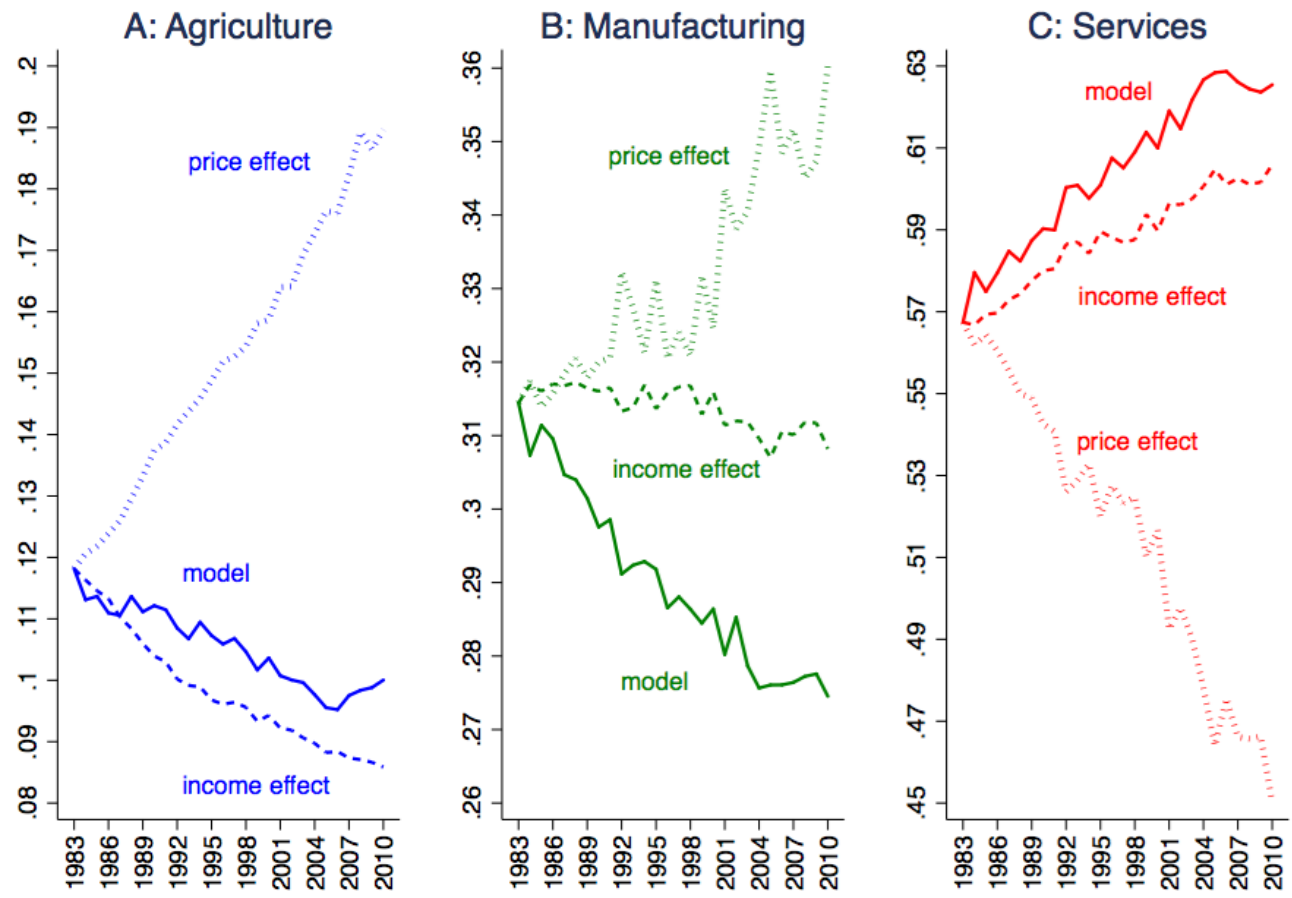

Figure 1.9: Weighted-average Aggregate Expenditure Shares and Income and Price Effects

Notes: This figure depicts the weighted-average aggregate expenditure shares (model) and their counterfactuals implied by the income effects and price effects in agriculture (Graph A), manufacturing (Graph B) and services (Graph C) using CE-BLS data. Solid lines, dashed lines and dotted lines denote, respectively, the model prediction, income effects and price effects. See equations $(10),(12)$ and (13) for the formal definitions of the weighted-average sectoral expenditure shares and the counterfactuals implied by the income effects and price effects, respectively.

Figure 1.9 depicts the sectoral weighted-average aggregate expenditure shares as predicted by equation (10) and the associated fits implied by the income effect (equation (12)) and price effects (equation (13)). We can intuitively see that price effects alone drive the expenditure shares in the opposite direction to income effects and to what is observed in the model prediction. Furthermore, consistently with Herrendorf et al. (2009), we see that the counterfactual implied by the income effects associated with the nonhomotheticities are the prevailing source of the observed structural change in the shares of consumption expenditure, especially in agriculture and services - sectors that feature nonhomothetic demands ${ }^{56}$.

${ }^{56} \mathrm{It}$ is particularly strong in agriculture. Furthermore, remind that the manufacturing expenditure shares reflect the nonhomotheticity of the other sectors, even though they feature a weaker income effect, by definition. 

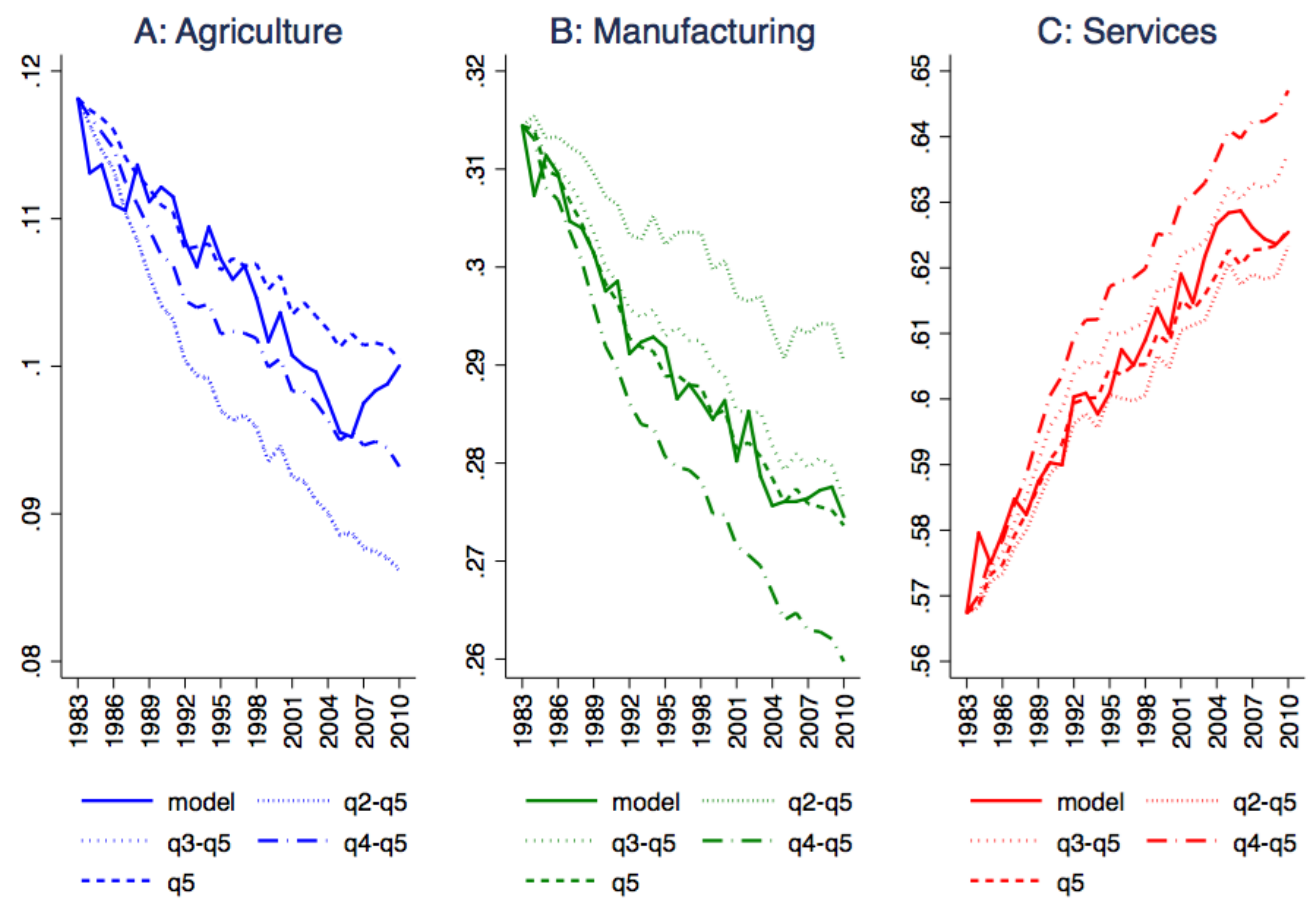

Figure 1.10: Weighted-average Aggregate Expenditure Shares and Income Effects by Selected Income Quintiles

Notes: This figure depicts the weighted-average aggregate expenditure shares (model) and their counterfactuals implied by selected quintiles' income effects in agriculture (Graph A), manufacturing (Graph B) and services (Graph C) using CE-BLS data. $q_{2}-q_{5}, q_{3}-q_{5}, q_{4}-q_{5}$ and $q_{5}$ stand for the income effects of the respective income quintiles, while the predicted expenditure shares of the remaining quintiles are held constant at their 1983 levels and the estimated quintile's weights changes as dictated by the data. See subsection 4.1 for the formal definitions of the quintiles' income effects.

We explore the income effects further by showing their relavance across households grouped by income quintiles. To this respect, we define the following four different counterfactual measures that stem from the fit of the weightedaverage aggregate expenditure shares implied by the income effect that follows equation (12). We aggregate income effects of income quintiles to highlight the marginal relevance of different quintiles' income effects to capture the changes in aggregate expenditure shares. The measures follow: i. income effects of quintiles $q_{2}-q_{5}$ - the expenditure share of quintile $q_{1}$ is held constant at $t_{0}=1983$ and the income effects of quintiles $q_{2}-q_{5}$ follow equation (12) (prices and total expenditure of $q_{1}$ held constant at $\left.t_{0}\right): \tilde{s}_{i, t}^{e_{q_{2}-q_{5}}} \equiv$ $\hat{\omega}_{q_{1}, t} \hat{s}_{q_{1}, i, t_{0}}+\sum_{k=2}^{5} \hat{\omega}_{q_{k}, t} \hat{s}_{q_{k}, i, t}^{e}$, where the superscript $e_{q_{2}-q_{5}}$ denotes income effects of quintiles $q_{2}-q_{5}$; ii. income effects of quintiles $q_{3}-q_{5}: \tilde{s}_{i, t}^{e_{q_{3}}-q_{5}} \equiv \sum_{k=1}^{2} \hat{\omega}_{q_{k}, t} \hat{s}_{q_{k}, i, t_{0}}+$ $\sum_{l=3}^{5} \hat{\omega}_{q_{l}, t} \hat{s}_{q_{l}, i, t}^{e}$; iii. income effects of quintiles $q_{4}-q_{5}: \tilde{s}_{i, t}^{e_{q_{4}-q_{5}}} \equiv \sum_{k=1}^{3} \hat{\omega}_{q_{k}, t} \hat{s}_{q_{k}, i, t_{0}}+$ $\sum_{l=4}^{5} \hat{\omega}_{q_{l}, t} \hat{s}_{q_{l}, i, t}^{e}$ and iv. income effect of quintile $q_{5}: \tilde{s}_{i, t}^{e_{q_{5}}} \equiv \sum_{k=1}^{4} \hat{\omega}_{q_{k}, t} \hat{s}_{q_{k}, i, t_{0}}+$ $\hat{\omega}_{q_{5}, t} \hat{s}_{q_{5}, i, t}^{e}$. Interestingly, as Figure 1.10 illustrates, the changes in the weighted- 
average aggregate expenditure shares (model) in all sectors are specially driven by the income effect of the top quintile $q_{5}$.

\section{4 .2}

\section{Counterfactual Experiment}

Remind that two aspects enable us to approach our central question on whether there is an empirical correlation between consumption inequality and structural change. First, we take benefit from a database of household expenditures associated to consumer prices indexes to disaggregate the sectoral expenditure shares by households grouped by income quintiles. However, expenditures reported in household surveys are potentially mis-measured. Then, we correct these systematic mis-measurements applying consolidated econometrical techniques (Aguiar and Bils (2011)) . Second, we apply a benchmark demand system of structural change in the cross-section of households so that it is consistent with canonical work that uses aggregate data Herrendorf et al. (2009). Here two more issues. First, if consumption (income) inequality concerns structural change it has to show up through income effects by definition. We show that income effects are the dominant source of the aggregate structural change in the shares of consumption expenditure. Second, we highlight that there are heterogeneous income effects across households grouped by income quintiles.

Up to this point we note that any possible correlation between consumption inequality and structural change in the past three decades in the US concerns specially the top quintile. With respect to consumption inequality, we show in subsection 3.1.3 that it is driven virtually exclusively by the quintile $q_{5}$, as suggest Figure 1.2. In regard to structural change, we saw in subsection 4.1 that the income effects of quintile $q_{5}$ captures the bulk of the changes of the sectoral expenditure shares, as Figure 1.10 depicts. Therefore, to the extent that services are luxuries and both agricultural and manufacturing goods are necessities for quintile $q_{5}$, as illustrates Figure 1.8, consumption inequality and structural change are potencially connected.

In this sense, how large is this correlation in the past three decades in the US? In this section, we address this question by running a counterfactual experiment, in which we assume that during the period 1983-2010 the consumption inequality, measured by the quintiles' weights in total expenditure, is held constant at the 1983 pattern. More precisely, we assume that the weighted-average aggregate expenditure shares, which are implied by the estimated paramameters in the intratemporal problem of the stand-in household of each income quintile, are under the counterfactual in which all market 
prices and quintiles' expenditures evolve as observed in the data, but quintiles' weights are kept constant at their $t_{0}=1983$ levels. Following equation (10), we define the counterfactual weighted-average aggregate expenditure share:

$$
\hat{s}_{i, t}^{c} \equiv \sum_{k=1}^{5} \hat{\omega}_{q_{k}, t_{0}} \hat{s}_{q_{k}, i, t}
$$

where the superscript $c$ denotes the counterfactual regarding consumption inequalilty.
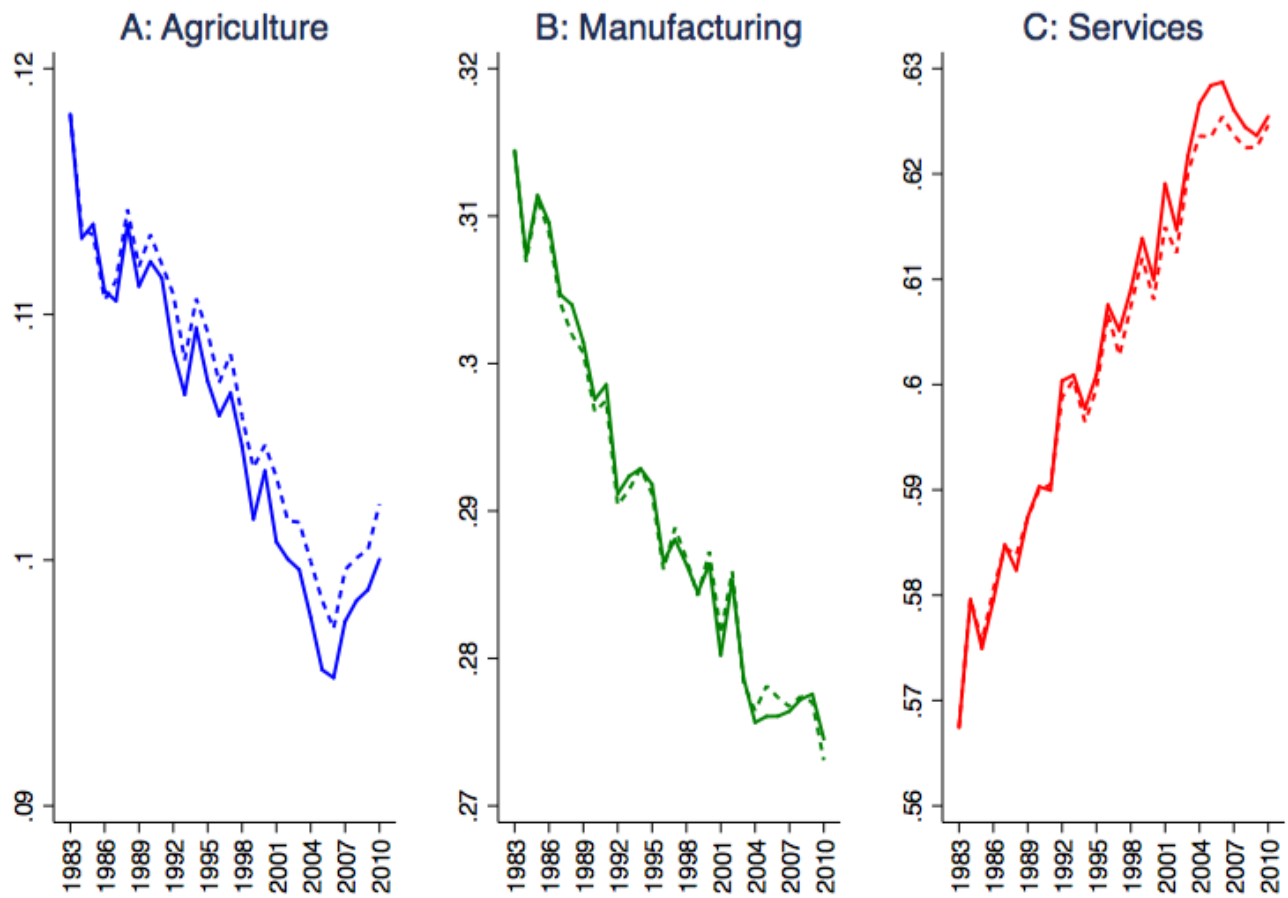

Solid line: model; Dashed line: model - counterfactual

Figure 1.11: Counterfactual experiment: No Increase in Consumption Inequality

Notes: This figure depicts the weighted-average sectoral expenditure share (model) in agriculture (Graph A), manufacturing (Graph B) and services (Graph C) and their counterfactuals in which all market prices and quintiles' expenditures evolve as observed in the data, but quintiles' weights are kept constant at their 1983 levels using CE-BLS corrected data. See equations (10) and (14), respectively, for the formal definitions of the weighted-average sectoral expenditure shares and their counterfactuals so that consumption inequality is held constant at the 1983 pattern.

Figure 1.11 depicts the weighted-average aggregate expenditure (model), defined in equation (10), and its trajectory under the counterfactual on consumption inequality (model - counterfactual) as defined in equation (14) for agriculture (Graph A), manufacturing (Graph B) and services (Graph C). According to our model, we see that if consumption inequality had kept constant since the beginning of the 1980's, there would have been less structural change in the past three decades in the US. That is, the expenditure shares of agriculture and manufacturing would be higher and, conversely, the expenditure 
share of services would be lower. For the expenditure share of manufacturing there is no clear change. It is worth mentioning that if we consider the counterfactual experiment described in equation (14) by considering the quintiles' weights implied by reported expenditure $\left(\omega_{q_{k}, t_{0}}\right)$, instead of considering the corrected quintiles' weights $\left(\hat{\omega}_{q_{k}, t_{0}}\right)$ defined in equation $(9)$, the weighted-average aggregate expenditure shares and their counterfactual trajectories would be virtually identical. That is, at first glance, by considering directly the reported expenditures in the CE survey it would suggest no empirical correlation between consumption inequality and structural change. See Figure A.10 in Appendix A.

A simple algebraic argument helps to synthesize our reduced-form sense of the empirical correlation between consumption inequality and structural change. We decompose the change of the wighted-average aggregate expenditure share of sector $i$ between any two periods $t_{1}$ and $t_{0}, \triangle \hat{s}_{i, t_{1}}$, into an extensive margin (change of the quintiles' expenditure weights) and an intensive margin (change of the quintiles' sectoral expenditure shares). Since consumption inequality increase, we define the income quintiles whose expenditure weights rise, $q_{k^{\prime \prime}}$, such that $k^{\prime \prime} \in \mathcal{K}^{\prime \prime}$ and the income quintiles whose expenditure weights fall, $q_{k^{\prime}}$, such that $k^{\prime} \in \mathcal{K}^{\prime}$. Yet, note that since the weights add up to one at any period $t$, we have that $\left|\triangle \hat{\omega}_{q_{k^{\prime}}, t}\right|=\left|\triangle \hat{\omega}_{q_{k^{\prime \prime}}, t}\right|$ and $\hat{\omega}_{\mathcal{K}^{\prime \prime}, t}=1-\hat{\omega}_{\mathcal{K}^{\prime}, t}$. Then the change of the wighted-average aggregate expenditure share of sector $i$ in $t_{1}$ is decomposed as follows:

$$
\begin{aligned}
\triangle \hat{s}_{i, t_{1}} & =\triangle \hat{s}_{\mathcal{K}^{\prime \prime}, i, t_{1}}+\triangle \hat{s}_{\mathcal{K}^{\prime}, i, t_{1}} \\
& =\left(\triangle \hat{\omega}_{\mathcal{K}^{\prime \prime}, t_{1}} \hat{s}_{\mathcal{K}^{\prime \prime}, i, t_{1}}+\triangle \hat{s}_{\mathcal{K}^{\prime \prime}, i, t_{1}} \hat{\omega}_{\mathcal{K}^{\prime \prime}, t_{0}}\right)+\left(\Delta \hat{\omega}_{\mathcal{K}^{\prime}, t_{1}} \hat{s}_{\mathcal{K}^{\prime}, i, t_{1}}+\triangle \hat{s}_{\mathcal{K}^{\prime}, i, t_{1}} \hat{\omega}_{\mathcal{K}^{\prime}, t_{0}}\right) \\
& =\left[\triangle \hat{\omega}_{\mathcal{K}^{\prime \prime}, t_{1}}\left(\hat{s}_{\mathcal{K}^{\prime \prime}, i, t_{1}}-\hat{s}_{\mathcal{K}^{\prime}, i, t_{1}}\right)\right]+\left[\left(\triangle \hat{s}_{\mathcal{K}^{\prime \prime}, i, t_{1}}-\triangle \hat{s}_{\mathcal{K}^{\prime}, i, t_{1}}\right) \hat{\omega}_{\mathcal{K}^{\prime \prime}, t_{0}}+\triangle \hat{s}_{\mathcal{K}^{\prime}, i, t_{1}}\right]
\end{aligned}
$$

where $\hat{\omega}_{\mathcal{K}^{\prime \prime}, t} \equiv \sum_{k^{\prime \prime} \in \mathcal{K}^{\prime \prime}} \hat{\omega}_{q_{k^{\prime \prime}}, t}$ is the sum of the quintiles' weights that increase, $\hat{s}_{\mathcal{K}^{\prime \prime}, i, t} \equiv \sum_{k^{\prime \prime} \in \mathcal{K}^{\prime \prime}}\left(\hat{\omega}_{q_{k^{\prime \prime}}, t} \sum_{k^{\prime \prime} \in \mathcal{K}^{\prime \prime}} \hat{\omega}_{q_{k^{\prime \prime}}, t}\right) \hat{s}_{q_{k^{\prime \prime}}, i, t}$ is the aggregate (average) expenditure share of sector $i$ of the quintiles whose weights increase and $\hat{s}_{\mathcal{K}^{\prime}, i, t} \equiv \sum_{k^{\prime} \in \mathcal{K}^{\prime}}\left(\hat{\omega}_{q_{k^{\prime}}, t} \sum_{k^{\prime} \in \mathcal{K}^{\prime}} \hat{\omega}_{q_{k^{\prime}}, t}\right) \hat{s}_{q_{k^{\prime}}, i, t}$ is the aggregate (average) expenditure share of sector $i$ of the quintiles whose weights decrease.

Note that since consumption inequality increase, $\Delta \hat{\omega}_{\mathcal{K}^{\prime \prime}, t_{1}}>0$, and $\hat{\omega}_{\mathcal{K}^{\prime \prime}, t_{0}}>0$ by definition. Moreover, since total quintiles' expenditure increase and our demand system features nonhomotheticity, $\triangle \hat{s}_{\mathcal{K}^{\prime}, i, t_{1}}$ and the term $\left(\hat{s}_{\mathcal{K}^{\prime \prime}, i, t_{1}}-\hat{s}_{\mathcal{K}^{\prime}, i, t_{1}}\right)$ is either positive or not depending on whether, respectively, $i=s$ or $i=\{a, m\}$. The term $\left(\triangle \hat{s}_{\mathcal{K}^{\prime \prime}, i, t_{1}}-\triangle \hat{s}_{\mathcal{K}^{\prime}, i, t_{1}}\right)$ equals zero if the demand system implies linear Engel curves in terms of expenditure shares. We follow a Stone-Geary linear demand system, but our Engel curves in terms 
of expenditure shares are nonlinear, as defines equation (3). Thus, this term is also either positive or not depending on whether, respectively, $i=s$ or $i=\{a, m\}$.

We see in Figure 1.11 that there is year-to-year moviment largely reflecting sampling error and then the results can be sensible to the choice of the base year. Thus, to mitigate this issue, Table 5 reports the percent change of the variation of the weighted-average aggregate expenditure share that is attributed to the correlation with the increase of consumption inequality between the first three-year period ${ }^{57} t_{0}=(1983-1985)$ and the last three-year period $t_{1}=(2008-2010)$ of the sample period, as the following definition:

$$
\hat{\varsigma}_{i, t_{1}} \equiv \frac{\left(\triangle \hat{s}_{i, t_{1}}-\triangle \hat{s}_{i, t_{1}}^{c}\right)}{\triangle \hat{s}_{i, t_{1}}} \times 100=\frac{\left[\triangle \hat{\omega}_{\mathcal{K}^{\prime \prime}, t_{1}}\left(\hat{s}_{\mathcal{K}^{\prime \prime}, i, t_{1}}-\hat{s}_{\mathcal{K}^{\prime}, i, t_{1}}\right)\right]}{\triangle \hat{s}_{i, t_{1}}} \times 100
$$

According to Table 5, the percent change of the variation of the weightedaverage aggregate expenditure share that is attributed to the correlation with the increase of consumption inequality in agriculture is the largest, 10.8, and in services is 1.8. In manufacturing it accounts for -2.3 . However, we argue this is particularly due to sampling error and mis-measurements associated to durable goods $^{58}$. For example, the same percent change between 1983-1985 and 20052007 for manufacturing is 1.7 and Table A.7 in Appendix A, which includes the same calculations using nondurables, reports 2.5 for manufacturing, which are figures qualitatively in line with that reported for agriculture share, which is another goods sector.

\footnotetext{
${ }^{57}$ We average over these three-year periods.
}

${ }^{58} \mathrm{As}$ we mentioned, durables obliterate cross-sectional differences of consumption patterns due to the fact that differences in expenditure across income quintiles do not necessarily align with differences in durable stocks and associated service flows. 
$\Delta$ Percent of the weighted-average aggregate expenditure share (Counterfactual experiment relative to the model prediction)

$$
\begin{aligned}
& \hat{\zeta}_{a,(08-10)} \\
& \hat{\zeta}_{m,(08-10)} \\
& \hat{\zeta}_{s,(08-10)}
\end{aligned}
$$

Table 1.5: Percent Change of the Variation of the Weighted-average Aggregate Expenditure Shares Attributed to the Correlation With the Increase of Consumption Inequality - 2008-2010/1983-1985

Notes: This table reports the percent change of the variation of the weighted-average aggregate sectoral share that is attributed to the correlation with the increase of consumption inequality, $\hat{\varsigma}_{i,(08-10)}$, between 2008-2010/1983-1985, where $i=\{a, m, s\}$, using CE-BLS corrected data. The effect is in percent change, which means it is relative to the size of the predicted change of the weighted-average aggregate sectoral shares. See equation (15) for the formal definition.

Before concluding, it is worth discussing some limitations of our reducedform (empirical) correlation between consumption inequality and structural change. First, in our main results there is an underlying assumption which states that a level difference of a corrected income quintile's weight in total expenditure with respect to the same income quintile's weight computed directly from reported expenditure does not imply a change of the true sectoral expenditure shares of that that income quintile. We thus assume they do not change although the corrected weights might have changed relative to the reported ones in the CE-BLS survey. Equivalently, we suppose that either a rise or a fall of the corrected mean (aggregate) expenditure of a quIintile imply an increase or decrease of the corrected quintile's sectoral expenditure proportionally to its reported sectoral expenditure shares. We argue that it is a conservative assumption due to nonhomotheticity of preferences so that it implies that our main results are a lower bound for the true correlation between the rise in consumption inequality and structural change.

Remind that the estimation methodology on consumption inequality yields a corrected measure of consumption inequality but does not speak directly to mean expenditure. In paticular, it omits the bottom income quintile and estimates relative expenditure for the remaining income quintiles, from which we construct the corrected weights of the income quintiles. To move from relative expenditure to aggregate we need to take a stand on expenditure for the omitted quintile. Note, for example, that nonhomotheticity implies that 
the services shares are increasing in expenditure. Thus, if the true expenditure of the bottom income quintile is larger (smaller) than its reported expenditure, then the services share of the bottom quintile, which is implied by the reported data, understates (overstates) its true expenditure share of services. The opposite applies in the case of either agriculture or manufacturing.

\section{A: Reported data}

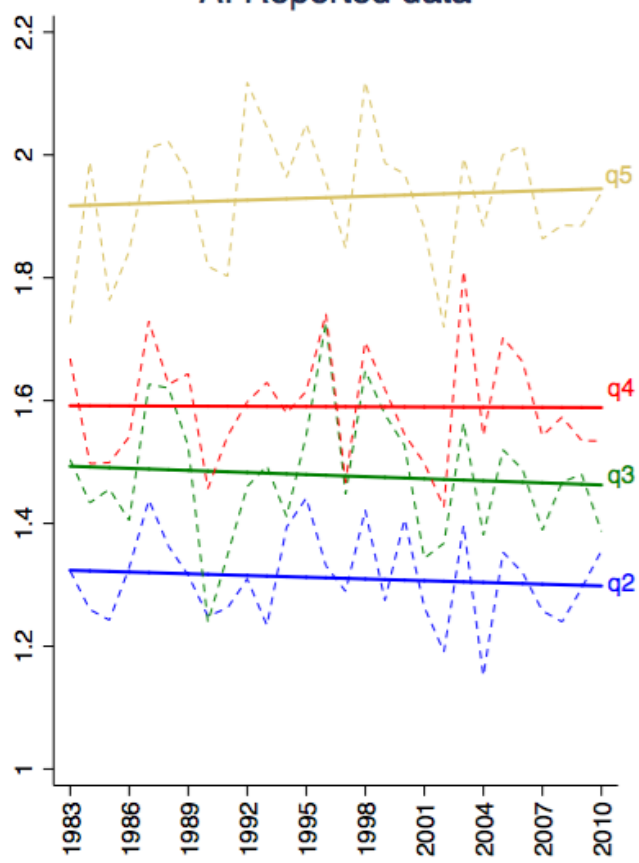

\section{B: Corrected data}

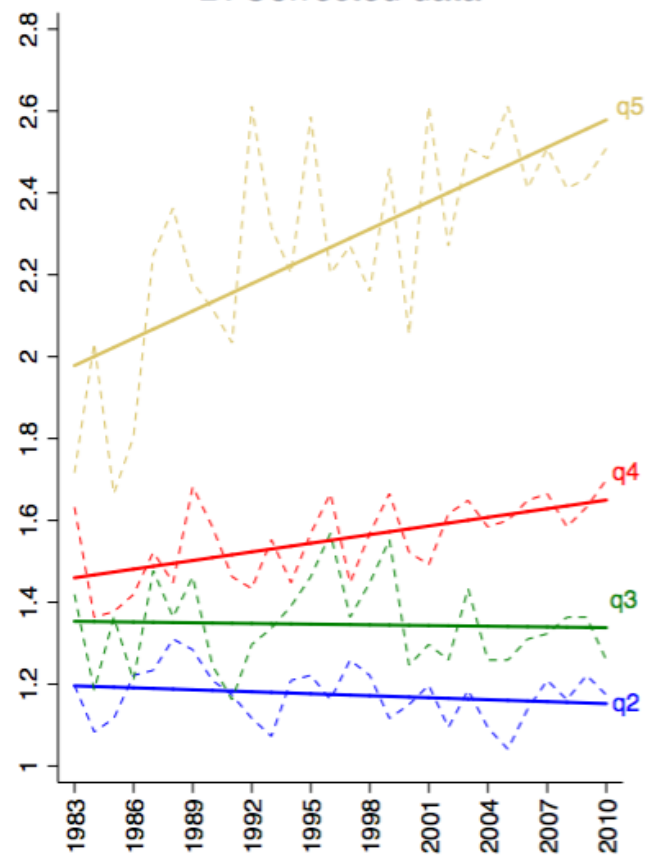

Solid line: linear trend; Dashed line: data

Figure 1.12: Trends in Consumption Inequality Based on Relative Expenditure Patterns Obtained From Reported Data in the CE Survey and Obtained From the Second-stage Regressions

Notes: This figure depicts the total expenditure ratio of each quintile versus the poorest income quintile (quintile 2-quintile 5: q2-q5) obtained from reported data in CE survey (Panel A) and obtained from the second-stage regressions (Panels B). Formally, the ratios represent the exp of the log of the relative growth in total expenditure for higher-income quintiles households relative to the lowest-income households for each year in the sample period, $\exp \left\{\delta_{q_{k}, t}\right\}, q_{k} \in\left\{q_{2}, q_{3}, q_{4}, q_{5}\right\}$ and $t=1983, \ldots, 2010$. Dashed lines and solid lines denote, respectively, data and linear trends.

For illustrative purposes, suppose expenditure is correctly reported for the bottom quintile. Figure 1.12 depicts the quintiles' relative (to bottom quintile) expenditures in reported data and corrected data and illustrates the arguments. By comparing Graphs A-B, we see that the reported relative expenditure of quintiles $q_{2}-q_{4}$ overstate their corrected ones, while the reported relative expenditure of quintile $q_{5}$ understates its corrected relative expenditure. Note that it then implies that $q_{2}-q_{4}$ overstate, for example, their services expenditue shares whereas quintile $q_{5}$ understates it due to nonhomotheticity. Moreover, when we understate the true expenditure share of 
the services sector of quintile $q_{5}$ (the quintile whose weight in total expenditure increase) and overstate the true services share of the remaining quintiles, it implies that by using the reported services expenditure shares, we understate the change of the weighted-average aggregate expenditure share of the services sector that is attributed to the correlation with the increase of consumption inequality. Thus, by comparing the level of the relative expenditures of each quintile in the Graphs, we see that the reported expenditure share of services in quintile $q_{5}$ surely understates its true one in the case the expenditure of the bottom quintile is correctly measured. Conversely, the reported services shares of $q_{2}-q_{4}$ surely overstate their true expenditure shares of the services sector. ${ }^{59}$

To this respect, a back-of-the-envelope calculation that assumes that expenditure is correctly reported for the bottom quintile and considers the relative expenditures, the corrected quintiles' weights and the expenditure shares elasticities of services reveals that our main result would understate the true correlation of consumption inequality and the expenditure share of services by about 4 percentage points between 1983-1985 and 2008-2010. Therefore, in this case, we argue that our main result would understate the true change of the weighted-average aggregate expenditure share of the services sector that is attributed to the correlation with the increase of consumption inequality.

\section{5 \\ Conclusion}

In this paper we estimate a standard model of structural change that features nonhomotheticity of preferences by using US household expenditure and price data over the period 1980-2010. We consider the aggregate sectoral expenditure shares as the composition of two margins of consumption expenditure: the extensive margin - a measure of consumption inequality that mirrors income inequality, expressed as the expenditure weights of households grouped by income quintiles - and the intensive margin - income quintiles' sectoral expenditure shares. Thus, we are able to estimate a measure of consumption inequality that corrects for systematic mis-measurements genneraly presented in reported expenditures in household expenditure surveys using a log-linear approximation to the Engel curves that stem from a linear expenditure system. Then analogously using a structural linear expenditure system, we estimate quintiles' sectoral expenditure shares in a consistent way with aggregate sectoral expenditure shares presented in recent evidence based on aggregate data from the National Income and Product Accounts.

\footnotetext{
${ }^{59}$ Again, the opposite applies in the case of either agriculture or manufacturing.
} 
We find that heterogeneity presented in the income effects of the structural change in the quintiles' expenditure shares is key to connect the rise of consumption inequality and structural change. In special, we show that the top quintile accounts for the bulk of the changes in both margins of consumption expenditure. Thus, we argue that to the extent that the top quintile drives the increase of consumption inequality and that for this quintile both agricultural and manufacturing goods are necessities and services are luxuries then the rise of consumption (income) inequality is potentially connected to structural change.

We then use the estimated model to perform a counterfactual experiment to measure the contribution of the rise of consumption inequality to structural change. We find that the increase of consumption (income) inequality correlates, although in a limited extent, with the changes in relative sectoral prices and, in special, the changes in aggregate income in accounting for the magnitude of structural change in the shares of consumption expenditure observed in the US in the past three decades. Particularly, we show that without the increase of consumption inequality, the rise of the aggregate expenditure share of services would be lower and, conversely, the fall of the aggregate expenditure shares of agriculture and manufacturing would be lower than the actual ones.

Possible extensions to the analysis we carried out in this paper are manifold. For example, in the demand system estimation, to characterize inflation at the household level will be usefull to assess whether the estimated structural parameters are sensible to the standard assumption which states that all households face the same prices. Moreover, as noted, we measure an empirical importance of the correlation between consumption inequality and structural change. We neither identify an exogenous causal effect of consumption inequality on structural change nor disentangle general equilibrium feedbacks. In this regard, it is of interest to extend our partial approach in a general equilibrium framework, for example, in heterogeneous agents and incomplete markets economies to study the nature of the reduced-form correlation we find in this empirical application. 


\section{Chapter 2}

\section{Reconciling Kuznets Facts, Engel's Law and Relative Prices trend in the postwar US in a Bewley-Aiyagari Framework with Stone-Geary Preferences}

\section{1 \\ Introduction}

Structural change was listed by Kuznets (1973) Kuznets (1973) as one of the six main features of modern economic growth. Thenceforth, recent work on the literature of structural change typically extends the standard one-sector neoclassical growth model to incorporate either supply or demand mechanisms to explain the change in the sectoral shares of consumption expenditures in time series - the so called Kuznets facts ${ }^{1}$ - through two main driving forces: changes in relative sectoral prices (price effects) - supply-side theories and changes in aggregate income (income effects) - demand-side theories. The former stresses in general two mechanisms behind relative prices changes: heterogeneous growth of sectoral technology (Ngai and Pissarides, 2007) and differences in sectoral capital intensities (Acemoglu and Guerrieri, 2006), while the latter focuses on the relation between income growth and nonhomotheticity in preferences engendering persistent differences in the slope of Engel curves of broad sectors ${ }^{2}$ (Kongsamut (1997)).

In addition to the Kuznets facts, illustrated in figure 1 - panel A for the postwar US, within demand-side reasons of structural change there is empirical evidence at the micro level supporting that household demand of goods and services follows persistent differences in the slope of Engel curves ${ }^{3}$ (Young (2012), Boppart (2014), Aguiar and Bils (2011), Comin et al. (2015)). That is, the richest (poorest) the household, the greatest (lowest) is her expenditure share of services (figure 1 -panel B). Therefore, this evidence generalizes the stylized fact which states that the expenditure share of food declines as

${ }^{1}$ It is also known as the Fisher-Clark-Kuznets thesis (Fisher, 1939; Clark, 1940; Kuznets, 1955).

${ }^{2}$ Broad sector in the literature of structural change in general means either goods (agriculture and manufacturing) and services sectors or the goods sector is split into agriculture and manufacturing sectors in addition to the services sector.

${ }^{3}$ We follow Comin et al. (2015) in defining Engel curves as the relationship between sectoral consumption shares and aggregate (real) consumption holding prices constant. 
Chapter 2. Reconciling Kuznets Facts, Engel's Law and Relative Prices trend in the postwar US in a Bewley-Aiyagari Framework with Stone-Geary Preferences

household income rises (Engel, 1857 - Engel's law) to aggregate expenditure shares of many consumption goods, a broader goods sector, and is augmented with highly income elastic demand for services. With respect to supply-side reasons of structural change, another stylized fact states that the relative price of goods declines as an economy grows and becomes increasingly specialized in services (Boppart, 2013) - see figure 2.
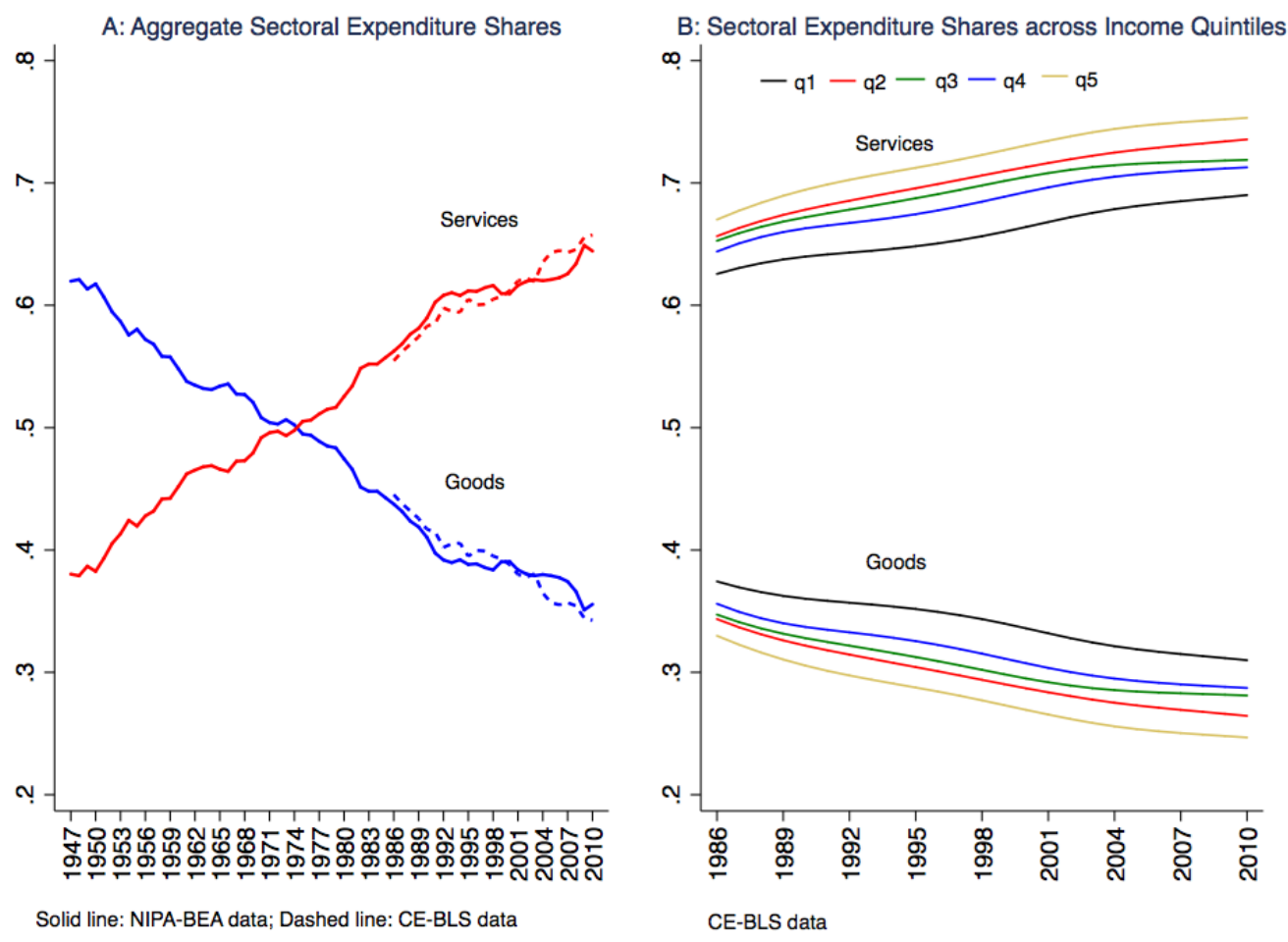

Figure 2.1: Kuznets Facts and Engel's Law in the Postwar US

Notes: This figure depicts in panel A the approximation of aggregate shares of consumption expenditure using data from NIPA-BEA in 1947-2010 and from CE-BLS in 1986-2010 for goods (agriculture and manufacturing) and services that follows Herrendorf et al. (2009) subject to smaller consistency issues due to data source differences - Kuznets facts. Solid and dashed lines denote, respectively, data from NIPA-BEA and data from CE-BLS. Panel B depicts the nondurable sectoral expenditure shares (smoothed with Hodrick-Prescott filter for easy of visualization) across income quintiles (quintile 1-quintile 5: $q_{1}-q_{5}$ ) using data from CE-BLS in 1986-2010 - Engel's law. Sectoral shares are defined by the ratio of sectoral expenditure to total expenditure. See the data appendix for further details on definitions of expenditure sectors. 


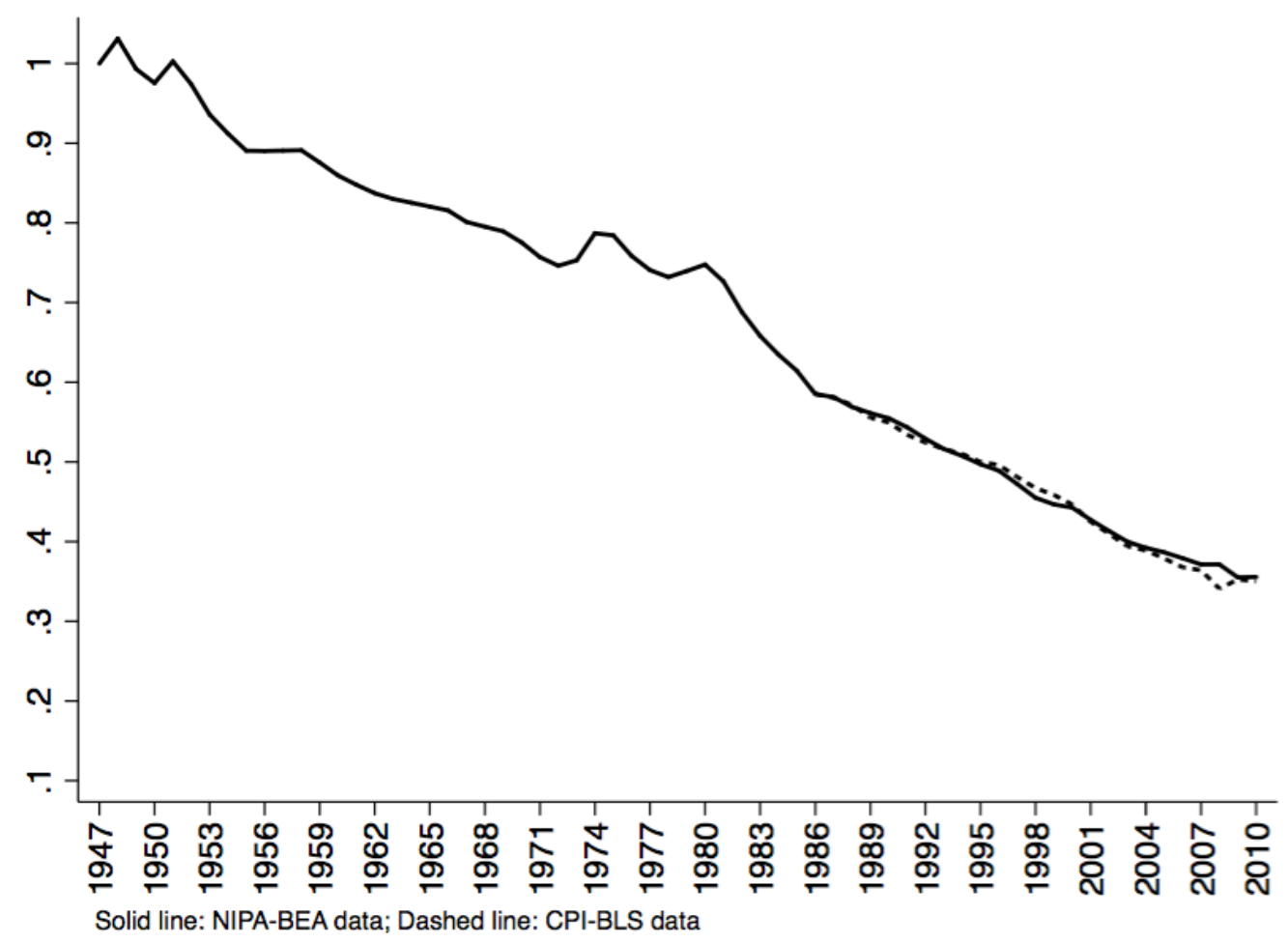

Figure 2.2: Goods to Services Prices Ratio in the Postwar US

Notes: This figure depicts the ratio of goods to services prices using data from NIPA-BEA and data from CPI-BLS for goods (agriculture and manufacturing) and services. Data from NIPA-BEA follows Herrendorf et al. (2009) subject to smaller consistency issues due to data source differences with CPI-BLS. Definition of sectors and its components with data from CPI-BLS matches to sectoral definitions with data from CE-BLS. Solid and dashed lines denote, respectively, data from NIPA-BEA and data from CPI-BLS. See the data appendix for further details on definitions of expenditure sectors.

Motivated by this evidence, we build a Bewley-Aiyagari style model (Hildenbrand et al. (1986), Aiyagari (1994)) in continuous time embedded with a two-sector environment and a demand-side benchmark theory relying on Stone-Geary preferences. Bewley-Aiyagari models constitute a natural workhorse to study heterogeneity in household choices and market outcomes altogether. Our application of the Bewley-Aiyagari model follows the framework of heterogeneous agent models in continuous time (HACT) in Achdou et al. (2017) that boils down to a system of two coupled partial differential equations, which imparts several computational advantages. It features a Hamilton-Jacobi-Bellman (HJB) equation, which characterizes households' optimal consumption expenditure and savings behavior given a stochastic process for labor income and a Kolmogorov Forward (KF) equation characterizing the evolution of the joint distribution of income and wealth given optimal choices of households ${ }^{4}$. This approach features a tractable algorithm that is

${ }^{4} \mathrm{HJB}$ and $\mathrm{KF}$ equations are coupled since optimal policies (expenditure and savings) depend on the interest rate which is determined in equilibrium and hence depends on the wealth distribution. 
based on a finite difference method that enables the computation of stationary and time-varying equilibria using efficient sparse matrix routines. It thus allows the characterization of the distribution of agents' optimal strategies and hence the distribution of the sectoral expenditure shares both in the steady state equilibrium and throughout the transition ${ }^{5}$. Therefore, representation of the generalized Engel's law, which we henceforth call Engel's Law for the brevity (Matsuyama (2019)), arises naturally by following demand patterns in the cross-section of households grouped by income per capita.

We carry out a numerical simulation that is able to illustrate the three empirical regularities, or stylized facts, of structural change that we highlight. We specify three versions of Stone-Geary preferences that feature the same level o aggregate capital in stationary equilibrium to stress the relevance of income and price effects for the representation of Kuznets facts and Engel's law in the model. The baseline case is a more flexible version of the Stone-Geary, which features relative prices and income effects and gives rise to a linear demand system across sectors, which, as we shall see, reasonably represents the observed behavior in data both in time series and in cross-sectional expenditure. The second specification follows Kongsamut et al. (1997), so that it only allows for income effects to take place in the model. Conversily, the third version of the model follows Ngai and Pissarides (2007) so that it only features price effects.

The model is calibrated to represent structural change in the aggregate goods and services expenditure shares, as illustrated by data from the National Income and Product Accounts of the Bureau of Economic Analysis - NIPABEA for the US postwar economy (1947-2010). This characterization in aggregate sectoral shares is consistent with cross-sectional demand patterns of households grouped by income quintiles from mid-80's, when there is data available of final-expenditure in the Consumption Expenditure Survey of the Bureau of Labor Statistics (CE-BLS). Therefore, these data sources are used to consistently reconciliate of the Kuznets facts with the Engel's law. It means that sectoral definitions of prices and final-expenditure in NIPA-BEA time-series data that follows Herrendorf et al. (2009) are subject to smaller consistency issues due to data source differences in the level of aggregation of household final-expenditure in the pooled cross-section data from CE-BLS

\footnotetext{
${ }^{5}$ Furthermore, this framework allows to extend the joint distribution of wealth and income in a more realistic way, which conveys important implications of public policy. For instance, it allows to extend recent policy analysis, such as in monetary policy in HANK models (Kaplan et. al, 2018) and fiscal policy in incomplete markets models (Heathcote, 2005; Kapalan and Violante, 2014) since it enables to assess not only direct effects and general equilibrium feedbacks of broad policies in an economy. It also admits general equilibrium assessement of policies targeted in specific disaggregated groups of households in different stages of structural change.
} 
Chapter 2. Reconciling Kuznets Facts, Engel's Law and Relative Prices trend in the postwar

between 1986 and 2010, which, in turn, is matched to a sectoral definition of prices from the Consumer Price Index (CPI-BLS) - see panel A of figure 1 and figure 2 .

The two main contributions of this study are in order. First, our general equilibrium framework in a multi-sector environment with nonhomothetic preferences enables us to show that price and income effects drive structural change not only through sectoral consumption demands stemming from intratemporal preferences. Relative prices and nonhomotheticity terms also drive structural change via intertemporal opportunities since they impact expenditure (and savings) policy functions. Second, the simulations with the three versions of the model suggest that the two driving forces of structural change in the aggregate shares of final consumption expenditure (Kuznets facts), price and income effects, are of roughly equal size in the postwar US. Moreover, we reinforce that reconciling these three main empirical regularities in the postwar US calls for a growth theory that accommodates both long-run demand (income effects) and supply (price effects) drivers of structural change.

The papers that are the closest to ours are Herrendorf et al. (2009) and Boppart (2014). The former has a partial equilibrium approach focused on the intratemporal problem of the households to explain the structural change on the aggregate expenditure shares of agriculture, manufacturing and services in the postwar US. We apply their Stone-Geary specification in two sectors and also their estimation methodology on data to feed the calibration of our general equilibrium model. To this respect, our paper can be considered a general equilibrium application of their partial approach, which, in addition, reconciles the Kuznets facts they depict in US aggregate data with the Engel's law at the micro level from mid-80's. That is, we argue that it is not necessary to depart from benchmark preferences to depict such reconciliation. For the sake of simplicity, in our framework households are ex-ante identical. It then implies that heterogeneity in sectoral expenditure patterns across household grouped by income quintiles arises exclusively from the idiosyncratic labor income (productivity) shocks. Herrendorf et al. (2009) also carry out a structural estimation to Stone-Geary preferences parameters in final-expenditure and prices postwar data from NIPA-BEA and perform simulations that suggest income effects are the dominant source of structural change in the aggregate shares of final consumption expenditure in the postwar US. Therefore, their result contrasts with ours in this matter. A key reason for this discrepancy is due to the differences in the level of sectoral aggregation. Herrendorf et al. (2009) show that by excluding government consumption from the services sector, which is more consistent to household expenditure, the estimated term 
of nonhomotheticity becomes lower, which implies that sectoral expenditure shares becomes less elastic to total expenditure and then it increases the explanatory power of relative prices to structural change.

Boppart (2014), in turn, was the first to square these three central stylized facts of structural change with balanced growth at the aggregate level, as described by the Kaldor facts, in a macroeconomic model with non-Gorman preferences that displays income and price effects. These effects are of equal importance for structural change in their empirical analysis for the postwar US, which is consistent to our result in this topic appling a different framework. The model in this paper features price-independent generalized-linear (PIGL) preferences and heterogenous agents through different initial labor and assets endownments in a complete markets environment. Since our focus is to match those three stylized facts of structural change to recent available data ranging from mid-80's, we are not inserted in this debate that rationalizes structural change along with aggregate Kaldor facts ${ }^{6}$. Therefore, unlike Boppart's model, which greatly depart from the benchmark model of structural change ${ }^{7}$, we keep close to benchmark demand systems of seminal papers in the literature as Ngai and Pissarides (2007)), Kongsamut et al. (1997) and Herrendorf et al. (2013) in a Bewley-Aiyagari framework. In this frame, contrary to Boppart (2014) , aggregation is trivial and computation of prices and aggregate and cross-sectional expenditure shares arises explicitly so that it enables us to match the data promptly. To this respect, being a tractable frame and easy to extend in many fronts, as for example, a more realistic income inequality theory, embedding distinct preferences to feature any specificity of demand patterns, etc., is an appealing feature of this framework relative to intrincate cutting-edges general equilibrium models ${ }^{8}$ applied to the structural change literature.

Besides this introduction, the paper is organized as follows. Section 2 presents the model; Section 3 conducts numerical simulations to illustrate the main stylized fact of structural change in the US from mid-80's. Section 4 concludes.

\footnotetext{
${ }^{6}$ See for example, Kongsamut et al. (1997); Ngai and Pissarides (2007) ; Acemoglu and Guerrieri, 2008; Foellmi and Zweimuller, 2008 and Boppart (2014) . Particularly Kongsamut et al. (1997) Kongsamut et al., 2001 and Ngai and Pissarides (2007) apply Stone-geary preferences featuring exclusively income effects and price effects, respectively, and show that under some special conditions it is possible to reconcile a balanced growth path with structural change in their models.

${ }^{7}$ That is, a multi-sector neoclassical growth model, as described by Herrendorf et al. (2013).

${ }^{8}$ See, for example, Buera and Kaboski (2009) and Boppart (2014)
} 


\section{2}

\section{Model Setup}

The model representation is an extension of the canonical one-sector Bewley-Aiyagari model described in Acemoglu and Jensen (2012) in which we apply rather usual features in a benchmark setup of structural change. We solve the model in continuous time building on Achdou et al. (2017) and we do not allow for rich heterogeneity across agents for simplicity. That is, utility functions, the distribution of labor endowments, and the lower bound on assets do not vary across households.

\subsection{1}

\section{Household Preferences}

There is an unit mass of infinitely lived households indexed by $h \in \mathcal{H}=$ $[0,1]$ that consume, borrow/save and inelastically supply labor. The continuum of households are heterogeneous in their wealth, $a$, and idiosyncratic labor productivity (measured in labor efficicient units), $z$, such that the joint distribution, $\mathfrak{g}(a, z, t)$, is the state of the economy at time $t$. Intertemporal preferences are (explicit) additively separeable over consumption (CRRA) specified over total consumption expenditure. Agreggate composite consumption follows Herrendorf et al. (2009) and adds agricultural to manufactured goods, which means we have a two-sector Stone-Geary demand system, so that indexes $\{g, s\}=J$, refer to the two broad sectors of goods and services, respectively.

As mentioned, a Bewley-Aiyagari framework constitutes a natural workhorse to study how changes in the distribution of economic parameters impact household choices and market outcomes, which is key to our analysis applied to structural change. In this respect, applying this framework enables us to characterize a joint wealth and income distribution in a more in-depth way. Solving the model in continuous time, in addition to imparting some notable computational advantages, provides a natural and parsimonious approach to modeling stochastic diffusion earnings process that generates kurtosis in data observed at discrete time intervals. Therefore, $z$ follows a stationary stochastic diffusion process (Ornstein-Uhlenbeck) that features a continuum of productivity types, a drift with a rate of mean reversion, $\mu=\zeta\left(z_{\text {mean }}-z_{t}\right)$, and a diffusion, $\sigma(z)$, that captures the size of innovations of a standard Brownian motion, $W_{t}$. This specification represents a continuous time analogue of an $\mathrm{AR}(1)$ process and encompasses (or differs only in minor ways from) the processes traditionally estimated in the literature.

It follows that in the class of models that features additively separable intertemporal preferences, the household may wish to save or to borrow 
according to the way in which it evaluates present and future needs, and this determines how much to allocate to current consumption expenditure, $e_{t}^{h}$, and in particular to (real) consumption commodities, $c_{j, t}^{h}$, where $j \in J$. Expenditure allocated to these commodities is the first stage in a two-stage allocation process - the intertemporal and intratemporal problems. The intertemporal problem follows closely, although slightly extends in a multi-sector environment in continuous time, the application of the Bewley-Aiyagari model in Acemoglu and Jensen (2012)

INTERTEMPORAL PROBLEM. Household $h$ makes a sequence of consumption expenditure decisions, $\left\{e_{t}\right\}_{t \geq 0}, e_{t} \in X \subseteq \mathbb{R}$, where $X$ is the household's action set, taking the sequence of wages, interest rates, price of the composite consumption $^{9}$ and sectoral prices $\left\{w_{t}, r_{t}, p_{t},\left\{p_{j, t}\right\}_{j \in J}\right\}_{t \geq 0}$ as given, by solving (we omit superscript $h$ henceforth for simplicity of notation ${ }^{10}$ ):

$$
\begin{gathered}
\max _{\left\{e_{t}\right\}_{t \geq 0}} \mathbb{E}_{0} \int_{0}^{\infty} \exp \{-\rho t\} \tilde{\nu}\left(e_{t}\right) d t \quad \text { s.t } \\
\tilde{\Gamma}\left(a_{t}, e_{t}, z_{t}, \mathrm{Q}_{t}, \boldsymbol{\theta}\right)= \begin{cases}\dot{a}_{t} & =w_{t}\left(\mathrm{Q}_{t}\right) z_{t}+r_{t}\left(\mathrm{Q}_{t}\right) a_{t}-e\left(\mathbf{p}_{\mathbf{t}}, \bar{u}_{t}\right) \\
d z_{t} & =\mu(z) d t+\sigma(z) d W_{t} \\
a_{t} & \geq \underline{a},\end{cases}
\end{gathered}
$$

where $\rho \in(0,1)$ is the descount factor; $\tilde{\nu}\left(e_{t}\right) \equiv \frac{\left(e_{t}\right)^{1-\varsigma}}{1-\varsigma}$ is the individual indirect utility function, such that $\varsigma>0$ is the inverse of the intertemporal elasticity of substitution (IES) of consumption expenditure; $a_{t}$ is the asset holdings of the household; $w_{t}\left(\mathrm{Q}_{t}\right)$ and $r_{t}\left(\mathrm{Q}_{t}\right)$ are respectively wages and interest rates; $\mathrm{Q}_{\mathrm{t}} \in$ $\mathcal{Q}$, such that $\mathcal{Q} \subseteq \mathbb{R}$, is the set of the market aggregate at time $t$ in Acemoglu and Jensen (2012) 's sense ${ }^{11} ; \boldsymbol{\theta} \in \Theta$, such that $\Theta \subseteq \mathbb{R}^{H}$, is a vector with the parameters; $e\left(\mathbf{p}_{\mathbf{t}}, \bar{u}_{t}\right) \equiv \sum_{j \in J} p_{j, t} c_{j, t}=p_{t} c_{t}-\sum_{j \in J} p_{j, t} \bar{c}_{j}$ is the household's total $^{12}$ consumption expenditure function, where $\mathbf{p}_{\mathbf{t}} \equiv\left\{p_{t},\left\{p_{j, t}\right\}_{j \in J}\right\}, \mathbf{p}_{\mathbf{t}} \in P$, such that $P \subseteq \mathbb{R}_{++}^{J+1}$, is the vector of relative prices and $\sum_{j \in J} p_{j, t} \bar{c}_{j}$ is a time varying endowment, such that $\bar{c}_{g}$ and $\bar{c}_{s}$ are constants (nonhomotheticity terms) that can be interpreted, respectively, as food subsistence and homeservices endowment (Kongsamut et al., 2001; Herrendorf et al. (2009); $\underline{a}$ is a lower bound on assets capturing both natural debt limits and other borrow

\footnotetext{
${ }^{9}$ Composite consumption is a sectoral consumption aggregator of the household.

${ }^{10}$ Except when we integrate a variable over the unit mass of households.

${ }^{11}$ See subsections 2.2 and 2.3 for our application of market aggregate and definition 1 in Acemoglu and Jensen (2012) for the formal definition. We mention here in advance that our market aggregate is the aggregate capital to "effective" labor ratio.

${ }^{12}$ Total - for all sectors in $J$.
} 
constraints $^{13}$. Note that $\underline{a}$ does not necessarily binds for a household even when the worst $z_{t}(\underline{z})$, occurs. Lower and upper bounds on assets are chosen so that they do not bind in equilibrium. Since $z \in Z$, such that we impose $Z=[\underline{z}, \bar{z}] \subseteq \mathbb{R}$, which follows an Ornstein-Uhlenbeck process and we also impose $X=[\underline{a}, \bar{a}] \subseteq \mathbb{R}$, it ensures compactness of productivities and actions. Moreover, it is well known that $\tilde{\nu}$ features standard continuity conditions ${ }^{14}$.

We rearrange the constraint so that we substitute $e_{t} \equiv e\left(\mathbf{p}_{\mathbf{t}}, \bar{u}_{t}\right)$ for $\dot{a}_{t} \equiv y_{t}$, the savings of each household. Since $\tilde{\nu}$ is increasing, we can solve for $e_{t}$ in terms of $y_{t}$ and rewrite decision problem:

$$
\begin{gathered}
\max _{\left\{y_{t}\right\}_{t \geq 0}} \mathbb{E}_{0} \int_{0}^{\infty} \exp \{-\rho t\} \tilde{\nu}\left(w_{t}\left(\mathrm{Q}_{t}\right) z_{t}+r_{t}\left(\mathrm{Q}_{t}\right) a_{t}-y_{t}\right) d t \quad \text { s.t } \\
\Gamma\left(a_{t}, z_{t}, \mathrm{Q}_{t}, \boldsymbol{\theta}\right)= \begin{cases}y_{t} \leq w_{t}\left(\mathrm{Q}_{t}\right) z_{t}+r_{t}\left(\mathrm{Q}_{t}\right) a_{t} \\
d z_{t} & =\mu(z) d t+\sigma(z) d W_{t} \\
a_{t} & \geq \underline{a},\end{cases}
\end{gathered}
$$

where $\nu\left(a_{t}, y_{t}, z_{t}, \mathrm{Q}_{t}, \boldsymbol{\theta}\right)=\tilde{\nu}\left(w_{t}\left(\mathrm{Q}_{t}\right) z_{t}+r_{t}\left(\mathrm{Q}_{t}\right) a_{t}-y_{t}\right)$. The intertemporal problem is seen to be a standard dynamic programming problem (Stokey and Lucas, 1989), with $\tilde{\nu}: X^{2} \times Z \times Q \times \boldsymbol{\theta} \rightarrow \mathbb{R}$ the instantaneous utility function and $\Gamma: X \times Z \times Q \times \boldsymbol{\theta} \rightarrow 2^{X}$ the constraint correspondence. A strategy $\mathbf{y}=\left(y_{1}, y_{2}, \ldots\right)$ is a sequence of random measurable maps $y_{t}: Z_{t-1} \rightarrow X$, where $Z_{t-1} \equiv \prod_{\iota=0}^{t-1} Z$, and gives rise to a joint distribution $\mathfrak{g}(a, z, t)$ for each $t$. A strategy is feasible if it satisfies $\Gamma$ in $(2)$ and it is optimal, $\mathbf{y}^{*}$, if it is a solution to $(2)$.

It is worth pointing out that the intertemporal problem in our multisector economy is the countinuous-time analogue of the decision problem in a (one-sector) standard Bewley-Aiyagari economy in discrete time. Apart from minimal adjustments to adapt for countinuous time, it generalizes the decision problem of the standard one-sector economy by considering relative prices and the expenditure function, which in the canonical one-sector version reduces to consumption due to consumption price normalization and homotheticity of preferences.

Intratemporal PROBlem. To allocate consumption expenditure in $t$

$13-\infty<\underline{a}<0$. In a stationary equilibrium with interest rate $r>0$, the natural debt limit is $\underline{a}=-\underline{z} r<0$, where $r>0$ and $\underline{z}$ is the lowest realization of $z_{t}$. Note that for any borrowing limit less tight than the natural borrowing limit, the constraint $-a<\underline{a}$ never binds.

${ }^{14}$ See assumption A.1 in Appendix B. 
among the two types of consumption commodities:

$$
\begin{gathered}
\min _{\left\{c_{j, t}\right\}_{j \in J}} \sum_{j \in J} p_{j, t} c_{j, t} \quad \text { s.t } \\
u\left(c_{g, t}, c_{s, t}\right) \geq \tilde{u}\left(\mathbf{p}_{\mathbf{t}}, e\left(\mathbf{p}_{\mathbf{t}}, \bar{u}_{t}\right)\right) \equiv\left[\sum_{j \in J} \varphi_{j}^{\frac{1}{\varepsilon}}\left(c_{j, t}+\bar{c}_{j}\right)^{\frac{\varepsilon-1}{\varepsilon}}\right]^{\frac{\varepsilon}{\varepsilon-1}},
\end{gathered}
$$

where $\left[\sum_{j \in J} \varphi_{j}^{\frac{1}{\varepsilon}}\left(c_{j, t}+\bar{c}_{j}\right)^{\frac{\varepsilon-1}{\varepsilon}}\right]^{\frac{\varepsilon}{\varepsilon-1}} \equiv c_{t}$ is a CES composite consumption (StoneGeary), such that $\varphi_{j}$ are nonnegative weights (add up to one) ${ }^{15}$. If all $\bar{c}_{j}$ are zero, then preferences are homothetic and $\varepsilon>0$ is the within-period elasticity of substitution between consumption categories.

Three comments are in order. First, since preferences are nonhomothetic, then $\varepsilon$ is not equal to the elasticity of substitution between consumption categories, which in practice means this specification does allow for differences in the elasticity of substitution between different pairs of consumption categories. Second, all households have preferences of the above form, which means households are ex-ante identical and only differs by following a shock drawn from the distribution of the individual-level stochastic productivity process. Third, considering that the utility function as well as the expenditure function feature standard conditions ${ }^{16}$ and that there is no corner solution, so that the dual problem applies, to solve problem (3) by minimizing expenditure $\sum_{j \in J} p_{j, t} c_{j, t} \equiv e\left(\mathbf{p}_{\mathbf{t}}, \bar{u}_{t}\right)$ subject to a utility level is equivalent to solve it by maximizing for composite consumption in $u\left(c_{t}\right)$ subject to total expenditure ${ }^{17}$.

It follows that applying Roy's identity, gives the sectoral uncompensated (Marshallian) demands, $c_{j}$, where $j \in J$.

$\frac{\partial e\left(\mathbf{p}_{\mathbf{t}}, \bar{u}_{t}\right)}{\partial p_{j, t}}=c_{j}\left(\mathbf{p}_{\mathbf{t}}, e\left(\mathbf{p}_{\mathbf{t}}, \bar{u}_{t}\right)\right)=\left[\frac{\varphi_{j}}{p_{j, t}^{\varepsilon}}\left(\sum_{i=g, s} \frac{p_{i, t}^{\varepsilon} \bar{c}_{i}}{\varphi_{i}}\right)-\bar{c}_{j}\right]+\left(\frac{\varphi_{j}}{p_{j, t}^{\varepsilon} \sum_{i=g, s} \varphi_{i} p_{i, t}^{1-\varepsilon}}\right) e_{t}$,

where we refer to $i=\{g, s\}$ here with a slight abuse of terminology. Note that under intertemporal separability, once $e_{t}$ is determined in the first stage, $c_{j, t}$ can be chosen without reference to prices and incomes outside $t$ (Blundell and Walker, 1986).

The expenditure share of $j$ in instant $t$ completes the representation of the households' preferences. We denote

\footnotetext{
${ }^{15}$ Note that in our two-sector environment, it then means $\varphi_{s}=1-\varphi_{g}$.

${ }^{16}$ They are taken to be continuous, diffentiable, increasing and strictly concave.

${ }^{17}$ It follows from the trivial equality $\nu\left(\mathbf{p}_{\mathbf{t}}, e\left(\mathbf{p}_{\mathbf{t}}, u_{t}\right)\right)=u$, such that $u=u\left(c_{t}\right)$. Without loss of generality, take the unconstrained case for simplicity. Applying the chain rule $\frac{d \nu\left(e\left(\mathbf{p}_{t}, c_{t}\right)\right)}{d c_{t}}=\frac{d \nu_{i}\left(e_{0}\right)}{d e_{t}} \cdot \frac{d e_{t}}{d c_{t}}=0$ if $e_{0}=\arg \max \nu$. Moreover, $\frac{d^{2} \nu\left(e\left(, \mathbf{p}_{t}, c_{t}\right)\right)}{d\left(c_{t}\right)^{2}}=\frac{d^{2} \nu\left(e_{0}\right)}{d\left(e_{t}\right)^{2}} \cdot\left(\frac{d e_{t}}{d c_{t}}\right)^{2} \leq 0$ since $\nu$ is concave.
} 


$$
s_{j}\left(\mathbf{p}_{\mathbf{t}}, e_{t}\right) \equiv \frac{p_{j, t} c_{j, t}}{\sum_{i=g, s} p_{i, t} c_{i, t}}=\frac{\varphi_{j} p_{j, t}^{1-\varepsilon}}{\sum_{i=g, s} \varphi_{i} p_{i, t}^{1-\varepsilon}}\left(1+\sum_{i=g, s} \frac{p_{i, t} \bar{c}_{i}}{e_{t}}\right)-\frac{p_{j, t} \bar{c}_{j}}{e_{t}},
$$

where it follows that the expenditure share of $j, s_{j, t}$, depends explicitly on relative prices and total consumption expenditure.

We will estimate the parameters of the utility function using (5) and use it to feed the model calibration in the numerical analysis we carry out in section 3 .

\subsection{2}

\section{Technology}

The supply side of the economy comprises all consumption sectors $j \in J$ and an investiment sector $d$, where each sector features a representative firm in perfect competition. In particular, suppose that each sectoral production function of the economy is given by a constant return to scale Cobb-Douglas

$$
Y_{m, t}=K_{m, t}^{\alpha}\left(A_{m, t} L_{m, t}\right)^{1-\alpha}
$$

where $m \in M \equiv\{d\} \cup J$ and $\alpha \in(0,1)$ denotes capital intensity, which means we do not allow for sector-specific capital intensities for simplicity. Since $\bar{L}_{t} \equiv \sum_{m} L_{m, t}=1$, is the total labor endowment of the economy, it implies that aggregate capital equals capital-labor ratio in any sector $m \in M$ :

$$
K_{t}=\frac{\alpha}{1-\alpha} \frac{w_{t}}{R_{t}}=\frac{K_{m, t}}{L_{m, t}} .
$$

It gives rise to a convenience in terms of aggregate output. That is, we can consider an aggregate production function that produces a single good that can be turned into either consumption or investment via a linear technology with marginal rate of transformation equal to $p_{t}$. Furthermore, the economy laboraumenting productivity becomes simply investment sector labor-aumenting productivity:

$$
Y_{t}=D_{t}+\sum_{j \in J} p_{j, t} c_{j, t}=K_{t}^{\alpha} A_{d, t}^{1-\alpha},
$$

where we normalize investment sector price, $p_{d, t}=1$, capital accumulates as usual, $\dot{K}_{t}=D_{t}-\delta K_{t}$, and $D_{t}$ is aggregate investment goods demand.

In this model, we define $Q_{t}$ as the aggregate variable - the effective capital-labor ratio

$$
Q_{t} \equiv \frac{K_{t}}{A_{d, t} \bar{L}_{t}}
$$

through which all markets interactions take place. Note that we can naturally define an aggregate production function as a function of $Q_{t}, Y_{t}=A_{d, t} Q_{t}^{\alpha}$. 
Hence, this market aggregate uniquely determines the wage and interest rate at $t$ via the usual marginal product conditions ${ }^{18}$

$$
w_{t}=(1-\alpha) A_{d, t} Q_{t}^{\alpha}, \quad r_{t}=\alpha Q_{t}^{\alpha-1}-\delta .
$$

From marginal product conditions and by equilizing the prices of labor and capital across sectors and by considering (7), we have that prices are determined by technology for any $j \in J$ :

$$
p_{j, t}=\left(\frac{A_{d, t}}{A_{j, t}}\right)^{1-\alpha} .
$$

Then some algebra manipulation by using the optimal conditions in the intratemporal problem of the households and by using the definition of $c_{t}{ }^{19}$, we have the price of the composite consumption:

$$
p_{t} \equiv\left[\sum_{j \in J} \varphi_{j}\left(p_{j, t}\right)^{1-\varepsilon}\right]^{\frac{1}{1-\varepsilon}} .
$$

Finally, we allow for heterogenous (and constant) growth of sectoral technology, for any $m \in M$ :

$$
A_{m, t}=A_{m, 0} \exp \left\{\psi_{m} t\right\},
$$

where $\psi_{m}$ is the technological growth rate of sector $m$.

\subsection{3}

\section{Aggregator and Market Clearing}

The aggregator determines (the market aggregate) $Q_{t}$ in the "reduced" version of the Bewley-Aiyagari model represented by (2) in the intertemporal problem, where $a_{t}^{h}$ is capital holdings of household $h$ in date $t$. We follow Acemoglu and Jensen (2012)'s aggregator definition, so that for all $t$,

$$
Q_{t}=\int_{0}^{1} a_{t}^{h} d h
$$

Therefore, it is the "average" of the strategies of agents ${ }^{20}$, which "cancels out" individual-level uncertainty (see, e.g., Lucas 1980; Bewley 1986; Aiyagari 1994). In stationary equilibrium, $A_{d} \bar{L}$ can be normalized to one so that our baseline aggregator (14), $Q_{t}=\int_{0}^{1} a_{t}^{h} d h A_{d, t} \bar{L}_{t}$, is equivalent to the capital market-clearing condition. In equilibrium, capital and labor markets clear, that is, for any sector $m \in M$ :

\footnotetext{
${ }^{18}$ Note that $R_{t}=r_{t}+\delta$ is the gross interest rate, such that $r_{t}=D_{K} Y_{t}$.

${ }^{19}$ See Herrendorf et al. (2009). It follows from some some algebra manipulation in the primal version of the intratemporal decision problem of the household by considering the definition of $c_{t}$ and the fact that the Lagrange multiplier is the marginal value of an additional unit of expenditure in period $t$.

${ }^{20}$ The integral is the Pettis integral (Uhlig, 1996).
} 


$$
\bar{L}_{t}=\sum_{m \in M} L_{m, t}=\int_{0}^{1} z^{h} d h, \quad \text { and } \quad K_{t}=\sum_{m \in M} K_{m, t}=\int_{\underline{z}}^{\bar{z}} \int_{\underline{a}}^{\infty} a \mathfrak{g}(a, z, t) d a d z .
$$

Let us write the aggregate demands as consumption goods (and services) demand, $C_{j, t}=\int_{\underline{z}}^{\bar{z}} \int_{\underline{a}}^{\infty} c_{j, t} \mathfrak{g}(a, z, t) d a d z$, and investment goods demand, $D_{t}=\int_{\underline{z}}^{\bar{z}} \int_{\underline{a}}^{\infty} y_{t} \mathfrak{g}(a, z, t) d a d z$. Then, market clearing in the consumption and investiment markets requires

$$
Y_{j, t}=C_{j, t}, j \in J \quad \text { and } \quad Y_{d, t}=D_{t}
$$

\subsection{4}

\section{Equilibrium}

\subsubsection{1}

\section{Transition dynamics}

Many equilibrium objects in this version of the model are nonstationary. To derive the normalized equilibrium we detrend the model's original equilibrium objects by investment sector productivity, which drives aggregate output growth in the long-run. To this respect, for any given object $o_{t}$, we have that $\hat{o}_{t} \equiv \frac{o_{t}}{A_{d, t}}$. Furthermore, the new discount rate becomes $\hat{\rho} \equiv \rho-(1-\gamma) \psi_{d}$. Thus, the detrended version of the model states equations $((1)-(16))$ in the previous subsections with detrended objects.

As we mentioned in the introduction, we follow the framework of the heterogeneous agent models in continuous time (HACT) - in Achdou et al. (2017). It boils down to a system of two coupled partial differential equations (PDEs). A Hamilton-Jacobi-Bellman (HJB) equation, which characterizes households' optimal consumption and saving behavior given a stochastic process for labor income and a Kolmogorov Forward (KF) equation characterizing the evolution of the joint distribution of income and wealth given optimal choices of households.

It is convenient to split the equilibrium definition into two related definitions: the dynamic equilibrium and the static equilibrium. The former relates to the intertemporal problem of the household and is essentially the same concept applied in a canonical Bewley-Aiyagari model with minimal modifications to accommodate a multi-sector economy. The latter is related to the intratemporal problem of the household.

The problem of individuals and the joint distribution of income and wealth satisfy HJB and KF equations for every $t$. Therefore, the dynamic equilibrium of this model can be represented by a set of normalized objects 
$v(\hat{a}, z, t), \mathfrak{g}(\hat{a}, z, t), \hat{w}_{t}, r_{t}, Q_{t},\left\{p_{j, t}\right\}_{j=\{g, s\}}, p_{t}$ and $A_{d, t}$, such that:

$$
\begin{aligned}
\hat{\rho} v(\hat{a}, z, t) & =\max _{\hat{y} \in \Gamma\left(\hat{a}_{t}, z_{t}, Q_{t}, \boldsymbol{\theta}\right)} \tilde{\nu}\left(w\left(Q_{t}\right) z_{t}+\left(r\left(Q_{t}\right)-\psi_{d}\right) \hat{a}_{t}-\hat{y}\right)+D_{\hat{a}} v(\hat{a}, z, t) y(\hat{a}, z, t) \\
& +D_{z} v(\hat{a}, z, t) \mu(z)+\frac{1}{2} D_{z z} v(\hat{a}, z, t) \sigma^{2}(z)+D_{t} \mathbb{E}_{t}[v(\hat{a}, z, t)] \\
D_{t} \mathfrak{g}(\hat{a}, z, t) & =-D_{\hat{a}}(y(\hat{a}, z, t) \mathfrak{g}(\hat{a}, z, t))-D_{z}(\mu(z) \mathfrak{g}(\hat{a}, z, t)) \\
& +\frac{1}{2} D_{z z}\left(\sigma^{2}(z) \mathfrak{g}(\hat{a}, z, t)\right) \\
p_{j, t} & =\left(\frac{A_{d, t}}{A_{j, t}}\right)^{1-\alpha}, j \in J, \quad p_{t}=\left[\sum_{j \in J} \varphi_{j}\left(p_{j, t}\right)^{1-\varepsilon}\right]^{\frac{1}{1-\varepsilon}} \\
r_{t} & =\alpha\left(Q_{t}\right)^{\alpha-1}-\delta, \quad \hat{w}_{t}=(1-\alpha)\left(Q_{t}\right)^{\alpha} \\
Q_{t} & \equiv \hat{K}_{t}=\int_{\underline{z}}^{\bar{z}} \int_{\hat{\underline{a}}}^{\infty} \hat{a} \mathfrak{g}(\hat{a}, z, t) d \hat{a} d z
\end{aligned}
$$

on $(\underline{\hat{a}}, \infty) \times(\underline{z}, \bar{z}) \times(0, T)$, where we apply Ito's lemma in (17) and (18), the HJB and KF equations, respectively.

The function $\hat{y}$ is the saving policy function

$$
\hat{y} \equiv y(\hat{a}, z, t)=\hat{w}\left(Q_{t}\right) z_{t}+\left(r\left(Q_{t}\right)-\psi_{d}\right) \hat{a}_{t}-e(\hat{a}, z, t),
$$

where the expenditure policy function

$$
\hat{e} \equiv e(\hat{a}, z, t)=p_{t} \hat{c}_{t}-\sum_{j \in J} p_{j, t} \hat{\bar{c}}_{j, t}=\left(\nu^{\prime}\right)^{-1}\left(D_{\hat{a}} v(\hat{a}, z, t)\right)
$$

arises from envelope condition in HJB.

The value function, $v$, satisfies a terminal condition. Although the time domain is $\mathbb{R}_{+}$, in practice the domain is $(0, T)$ for large $T$. Thus, it is imposed that

$$
v(\hat{a}, z, T)=v_{\infty}(\hat{a}, z)
$$

where $v_{\infty}$ is the stationary value function that solves the stationary analogue of the system $(17)-(21)$, below in equations $(27)-(28)$. The value function also satisfies a state constraint boundary condition at $\hat{a}_{t}=\underline{\hat{a}}_{t}$, which satisfies the following boundary conditions, for all $z$ :

$$
\tilde{\nu}^{\prime}\left(\hat{w}\left(Q_{t}\right) z_{t}+\left(r\left(Q_{t}\right)-\psi_{d}\right) \underline{\hat{a}}_{t}+\sum_{j \in J} p_{j, t} \hat{\bar{c}}_{j, t}\right) \leq D_{\hat{a}} v(\underline{\hat{a}}, z, t) .
$$


Since the diffusion is reflected at $\underline{z}$ and $\bar{z}$, the value function also satisfies the boundary conditions, for all $\hat{a}$ :

$$
D_{z} v(\hat{a}, \underline{z}, t)=0, \quad D_{z} v(\hat{a}, \bar{z}, t)=0 .
$$

We are now ready to define a dynamic equilibrium in this economy.

Definition 1 (Dynamic Equilibrium). Given initial conditions $\left(\hat{a}_{0}, z_{0}\right) \sim \mathfrak{g}(\hat{a}, z, 0)$ and a time path of sectoral productivities $\left\{\mathbf{A}_{m}\right\}_{m \in M}=$ $\left\{\left\{A_{m, t}\right\}_{m \in M}\right\}_{t \geq 0}, \quad$ a dynamic equilibrium is an evolution of prices $\left\{\hat{\mathbf{w}}, \mathbf{r}, \mathbf{p},\left\{\mathbf{p}_{j}\right\}_{j \in J}\right\}=\left\{\hat{w}_{t}, r_{t}, p_{t},\left\{p_{j, t}\right\}_{j \in J}\right\}_{t \geq 0}$, a time path of market aggregates and a strategy for each household, $\left\{\mathbf{Q}^{*}, \hat{\mathbf{y}}^{*}\right\}=\left\{\hat{Q}_{t}^{*}, \hat{y}_{t}^{*}\right\}_{t \geq 0}$, value functions $\boldsymbol{v}=\{v(\hat{a}, z, t)\}_{t \geq 0}$ and distributions $\mathfrak{g}=\{\mathfrak{g}(\hat{a}, z, t)\}_{t \geq 0}$, such that the following conditions hold: $i$. Household intertemporal optimality: $\boldsymbol{v}$ and $\mathfrak{g}$ satisfy the PDEs (17) and (18), considering boundary conditions (25) and (26), given $\mathbf{Q}^{*},\left\{\hat{\mathbf{w}}, \mathbf{r}, \mathbf{p},\left\{\mathbf{p}_{j}\right\}_{j \in J}\right\}$ and the initial conditions $\left(\hat{a}_{0}, z, 0\right) \sim \mathfrak{g}(\hat{a}, z, 0) ; i i$. Firms optimality; iii. Market clearing conditions (14) - (16).

Once we find the solution for the intertemporal problem, we properly compute the sectoral demands and expenditure shares following equations (4) and (5). Moreover, note that we assume interior solution in our framework. According to Herrendorf et al. (2009), although nonhomotheticity terms in this class of utility functions can lead to corner solution, we illustrate in the calibrated model in section 3 that each household chooses quantities that are far away from corners, which reflects the fact that it is not relevant for aggregate consumption in a rich country such as the postwar United States. Nevertheless, we have to impose an assumption in order to guarantee no corner solution. It means in our case that services consumption expenditure must be positive and then household's income must exceed a minimum level bounded by the time varying endowment ${ }^{21}$ :

Assumption 1. For every instant of time, the nonhomothetic terms $\hat{\bar{c}}_{a, t}$ and $\hat{\bar{c}}_{a, t}$ satisfy:

$\varrho\left(1-p_{s, t}^{1-\epsilon}\right)\left[p_{s, t} \max \left(-\hat{\bar{c}}_{s}, 0\right)\right]+\left(\frac{\varrho}{\varrho-1}\right)\left[p_{g, t} \max \left(-\hat{\bar{c}}_{g}, 0\right)\right] \leq\left(\hat{w}\left(Q_{t}\right) \underline{z}+\left(r\left(Q_{t}\right)-\psi_{d}\right) \underline{\hat{a}}\right)$,

where $\varrho \equiv\left[1+\frac{\varphi_{g}}{\varphi_{s}}\left(\frac{p_{g, t}}{p_{s, t}}\right)^{1-\varepsilon}\right]$.

Finally, we define a static equilibrium associated to a dynamic equilibrium:

Definition 2 (Static Equilibrium). Given objects of the dynamic equilibrium, a static equilibrium for each instant $t$ is defined as sectoral consumption demands for each household, $\left\{\hat{c}_{j, t}\right\}_{j \in J}$, and sectoral factor alloca-

\footnotetext{
${ }^{21}$ See appendix $\mathrm{B}$ for the derivation of assumption 1.
} 
tions $\left\{\hat{L}_{m, t}, \hat{K}_{m, t}\right\}_{m \in M}$, such that the following conditions hold: $i$. Household intratemporal optimality: for each household, $\left\{\hat{c}_{j, t}\right\}_{j \in J}$ follow (4) considering assumption 1; ii. Firms optimality; iii. Markets clear.

\subsubsection{2}

\section{Stationary equilibrium}

As we state in (24), we impose a terminal condition for the value function, $v$, in the dynamic equilibrium from which we solve backward. Formally, the problem of households and the joint distribution of income and wealth satisfy the stationary analogues of the HJB and KF equations (17) and (18), respectively:

$$
\begin{aligned}
\hat{\rho} v(\hat{a}, z) & =\max _{\hat{y} \in \Gamma(\hat{a}, z, Q, \boldsymbol{\theta})} \tilde{\nu}\left(\hat{w}(Q) z+\left(r(Q)-\psi_{d}\right) \hat{a}-\hat{y}\right)+D_{\hat{a}} v(\hat{a}, z) y(\hat{a}, z) \\
& +D_{z} v(\hat{a}, z) \mu(z)+\frac{1}{2} D_{z z} v(\hat{a}, z) \sigma^{2}(z) \\
0 & =-D_{\hat{a}}(y(\hat{a}, z) \mathfrak{g}(\hat{a}, z))-D_{z}(\mu(z) \mathfrak{g}(\hat{a}, z)) \\
& +\frac{1}{2} D_{z z}\left(\sigma^{2}(z) \mathfrak{g}(\hat{a}, z)\right)
\end{aligned}
$$

on $(\underline{\hat{a}}, \infty) \times(\underline{z}, \bar{z})$, such that the equilibrium objects, $(19)-(21)$, follow a constant time path and the policy functions (22) and (23) and boundary conditions $(25)-(26)$ follow their stationary analogues.

Note that, at the individual level, a stationary strategy means in our model that, for all $t, \hat{y}_{t}^{*}$ has the same joint distribution $\mathfrak{g}(\hat{a}, z) \in \mathfrak{G}(X, Z)$ and, at the aggregate level, $Q^{*}$ has a constant time path. Therefore, we have the following definition:

Definition 3 (Stationary Equilibrium). Given initial conditions $\left(\hat{a}_{0}, z_{0}\right) \sim \mathfrak{g}(\hat{a}, z, 0)$ and a constant time path of sectoral productivities $\left\{A_{m}\right\}_{m \in M}$, a stationary equilibrium is a constant evolution of prices, $\left\{\hat{w}, r, p,\left\{p_{j}\right\}_{j \in J}\right\}$, a constant time path of market aggregate and a stationary strategy with invariant distribution $\mathfrak{g}(\hat{a}, z)$ for each household, $\left\{Q^{*}, \hat{y}^{*}\right\}$, where $Q^{*}=\int_{\underline{z}}^{\bar{z}} \int_{\underline{\hat{a}}}^{\infty} \hat{a} \mathfrak{g}(\hat{a}, z) d a d z$ and $y(\hat{a}, z)$ is given by the stationary analogue of (22) and a stationary value function (24), such that the following conditions hold: $i$. Household intertemporal optimality - $v$ and $\mathfrak{g}$ satisfy the PDEs (28) and (29), considering the stationary analogues of the boundary conditions (25) and (26), given $Q^{*},\left\{\hat{w}, r, p,\left\{p_{j}\right\}_{j \in J}\right\}$ and the initial conditions $\left(\hat{a}_{0}, z_{, 0}\right) \sim \mathfrak{g}(\hat{a}, z, 0)$; ii. Firms optimality; iii. Markets clear.

It immediately follows from definition 3 (static equilibrium) that a constant time path of sectoral consumption demands and factor allocations 
stem from the stationary dynamic equilibrium due to the dependence of the static equilibrium on $\left\{Q^{*}, \hat{y}^{*}\right\}$.

Note in addition that we impose, as mentioned, a terminal condition as stationary equilibrium to solve the model numerically ${ }^{22}$. To this respect, the following assumption applies to the growth rates of sectoral technology, nonhomothetic terms and debt limit.

Assumption 2. Consider the growth rates of sectoral technology, $\left\{\psi_{m, t}\right\}_{m \in M}$, the nonhomothetic terms, $\left\{\bar{c}_{j}\right\}_{j \in J}$, an arbritrarily large terminal instant $T$ and an instant $\tau$, such that $0 \leq \tau \leq T$, and the debit limit $\underline{a}_{t}$. Then the following conditions must hold:

1. $\left\{\psi_{m, t}\right\}_{m \in M} \in \Psi$, such that $\Psi \subseteq \mathbb{R}^{M}$, are potencially different for $0 \leq t \leq$ $\tau$. Then $\left\{\psi_{m, t}\right\}_{m \in M}$ start to converge expontentially for $\tau<t<T$ and finally become equal for $T \leq t$.

2. $\left\{\bar{c}_{j, t}\right\}_{j \in J}$ grow at $\psi_{d}$, the economy (long-run) labor-aumenting productivity: $\left\{\hat{\bar{c}}_{j, t}\right\}$ become constant for $T \leq t$.

3. $\underline{a}_{t}=\underline{a}_{0} \exp \left\{\psi_{d}\right\}$, such that $\underline{\hat{a}}_{t}$ is constant.

We leave $\left\{\psi_{m, t}\right\}_{m \in M}$ free for $0 \leq t \leq \tau$ to calibrate the model according to target stylized facts of structural change. Yet, by setting $T$ sufficiently large, we are able to obtain a stationary system $(27)-(28)$ that implies aggregate expenditure share of services arbitrarily close to 1 (aggregate expenditure share of goods close to 0 ) and $\left\{\hat{\bar{c}}_{j, t}\right\}_{j \in J} \rightarrow 0 .{ }^{23}$ Note also that since $\left\{\psi_{m, t}\right\}_{m \in M}=\psi_{d}$ for $T \leq t$, the model admits a balanced growth path by construction from this point onwards. That is, as the detrended model converges to the stationary equilibrium, output, consumption, aggregate capital, wages and interest rate are constant ${ }^{24}$.

In a stationary equilibrium, each household faces a constant time path of aggregates, $Q^{*}$, and solves a stationary dynamic programming problem (27) - (28). HJB equation has a unique solution $v$, which is ensured by compactness of productivities and actions, and standard continuity conditions of the utility function. Furthermore, when the idiosyncratic shock process $z_{t}$ is stationary, $\mathfrak{g}(\hat{a}, z)$, in definition 3 , is an invariant distribution for the

${ }^{22}$ We overcome it by following Ribeiro (2018), which, in turn, follows Caselli and Coleman (2001).

${ }^{23}$ Note that the real value of the nonhomotheticity terms converging to zero asymptotically, $\left\{\hat{\bar{c}}_{j, t}\right\}_{j \in J} \underset{t \rightarrow+\infty}{\rightarrow} 0$, reveals that the Stone-Geary preferences exhibit asymptotic homotheticity.

${ }^{24}$ To see this, it suffices to show that $K_{t}, Y_{t}$ and $D_{t}$ all grow at rate $\psi_{d}$. The fact that $r_{t}$ is constant and equation (10) holds implies: $\dot{A}_{d, t}=\dot{K}_{t}=\psi_{d}$. Using $Y_{t}=A_{d, t}^{1-\alpha} K_{t}^{\alpha}$, we have: $\dot{Y}_{t}=\psi_{d}$. Constant growth of $K$ necessarily implies constant growth of $D$. 
Chapter 2. Reconciling Kuznets Facts, Engel's Law and Relative Prices trend in the postwar

households' decision problem. One can show that existence of such invariant distribution and stationary strategies are ensured by minimal and natural conditions ${ }^{25}$.

Proposition 1 (Existence of stationary equilibrium). Consider the Bewley-Aiyagari multi-sector model and suppose assumptions $1-2$ hold. Then there exists a stationary equilibrium.

The proof of proposition $1^{26}$ hinges upon the proof of theorem 2 in Acemoglu and Jensen (2012) on existence of a dynamic equilibrium, standard results on existence of a consumer demand correspondence related to equilibrium (See, e.g., Debreu, 1959 and Kreps, 2012) and on Topkis's theorem (Topkis, 1998).

\section{3}

\section{Numerical Simulation}

In this section we bring the model to the data to study how it performs in reconciling the three empirical regularities related to structural change in the US postwar period. We embed three benchmark specifications of Stone-Geary preferences, that Herrendorf et al. (2009) estimate in a partial approach focused in the intratemporal household problem, into the standard growth model in a Bewley-Aiyagari framework presented in section 2 to reconcile the trend of relative prices of goods with Kuznets Facts and Engel's law. We also follow these authors in performing structural estimation of preferences parameters to feed the model calibration using postwar NIPA-BEA data, between 19472010, although unlike that study, our sectoral definitions are subject to smaller consistency issues due to data source differences in the level of aggregation in household final-expenditure data from CE-BLS since 1986. CE-BLS data are drawn from the database prepared by Aguiar and Bils (2015) and represents the households' cross-sectional patterns of sectoral expenditures, so that we have a model calibrated to fit the Kuznets facts in the US postwar that are consistent with the Engel's law since mid-80's, which is the decade from which there is available data yearly.

Our baseline Stone-Geary specification follows the most flexible case that features both income and price effects, which is presented in section 2 in the intratemporal household problem (3) and gives rise to expenditure shares

\footnotetext{
${ }^{25}$ See Acemoglu and Jensen (2012) for further details.

${ }^{26}$ The proof is in Appendix B.
} 
Chapter 2. Reconciling Kuznets Facts, Engel's Law and Relative Prices trend in the postwar

$(5)^{27}$ - we present below as baseline case 1 . Cases 2 and 3 follow Herrendorf et al. (2013) so that they stress exclusively income effects and price effects, respectively. Instead of estimating parameters of each of the three Stone-Geary specifications, we only estimate parameters of the baseline model to assess marginally how income effects and price effects affect the model performance in terms of matching the three highlighted stylized facts of structural change in the postwar US. It means in cases 2 and 3 the preference parameters of the Stone-Geary specification are the same as in case 1 (baseline) apart from the following assumptions in terms of preferences (and technology) that we point out below.

Case 2 corresponds to the analysis found in Kongsamut et al. (1997) and represents the extreme scenario in which all structural change is driven by income effects that are generated by the non-homotheticity term $\bar{c}_{g}$ when income changes but prices are set fixed. It means we assume that technological progress is uniform across all consumption sectors $\left(\psi_{s, t}=\psi_{g, t} \forall t\right)$ and equals to the economy's long-run technological progress $\left(\psi_{d, t}\right)$, and that the parameter governing the elasticity of substitution among consumption goods is unity $(\varepsilon=1)$. Thus, the expenditure shares take the following form ${ }^{28}$ :

$$
s_{j}\left(\mathbf{p}_{\mathbf{t}}, \hat{e}_{t}\right)=\varphi_{j}\left(1+\sum_{i=g, s} \frac{p_{i, t} \hat{\bar{c}}_{i}}{\hat{e}_{t}}\right)-\frac{p_{j, t} \hat{\bar{c}}_{j}}{\hat{e}_{t}}
$$

Case 3 corresponds to the analysis found in Ngai and Pissarides (2007) ${ }^{29}$. The authors consider the polar extreme case in which structural change is generated purely from changes in relative prices. Accordingly, they assume the non-homotheticity terms are zero, $\bar{c}_{j}=0$. Therefore, for the structural change to arise it is clearly necessary to exist differential rates of technological progress among goods and services. Consistently to the empirical literature in structural change ${ }^{30}$, we impose $\psi_{g, t}>\psi_{s, t}$. In this case, the expenditure shares

${ }^{27}$ Note that we compute aggregate shares. It means that equation (5) depends on aggregate total expenditure, $s_{j}\left(\mathbf{p}_{\mathbf{t}}, \hat{E}_{t}\right)=\frac{\varphi_{j} p_{j, t}^{1-\varepsilon}}{\sum_{i=g, s} \varphi_{i} p_{i, t}^{1-\varepsilon}}\left(1+\sum_{i=g, s} \frac{p_{i, t} \bar{c}_{i}}{\hat{E}_{t}}\right)-\frac{p_{j, t} \bar{c}_{j}}{\hat{E}_{t}}$, where $\hat{E}_{t}=\int_{\underline{z}}^{\bar{z}} \int_{\underline{a}}^{\infty} \hat{e_{t}} \mathfrak{g}(\hat{a}, z, t) d a d z$. For each income quintile $q_{k}$, such that $k=\{1,2,3,4,5\}$, we define an income distribution $\mathfrak{g}_{\mathfrak{i}}(\hat{a}, z, t)$, where it is ordered from the bottom to top income, $\mathfrak{i}_{t}=\hat{w}_{t} z+r_{t} a$, where $z \in[\underline{z}, \bar{z}]$ and $a \in[\underline{a}, \bar{a}]$. Hence, aggregate expenditure of income quintile $q_{k}$ is $\hat{E}_{q_{k} t}=\int_{\underline{z}}^{\bar{z}} \int_{\underline{a}}^{\infty} \hat{e}_{t} \mathfrak{g}_{\mathfrak{i}_{q_{k}}}(\hat{a}, z, t) d a d z$, where $\mathfrak{g}_{\mathfrak{i}_{q_{k}}}(\hat{a}, z, t)$ is the density of income quintile $q_{k}$.

${ }^{28}$ The composite consumption in (3) becomes $\hat{c}_{t} \equiv \sum_{j \in J} \varphi_{j} \log \left(\hat{c}_{j, t}+\bar{c}_{j}\right)$ and then the following Marshallian demands: $c_{j}\left(\mathbf{p}_{\mathbf{t}}, \hat{e}\left(\mathbf{p}_{\mathbf{t}}, \bar{u}_{t}\right)\right)=\left[\left(\frac{\varphi_{j}}{p_{j, t}}\right) \sum_{i=g, s} p_{i, t} \hat{\bar{c}}_{i}-\hat{\bar{c}}_{j}\right]+\left(\frac{\varphi_{j}}{p_{j, t}}\right) \hat{e}_{t}$. Composite consumption price also becomes $p_{t}=\sum_{j \in J} \varphi_{j} \log p_{i t}$.

${ }^{29}$ This paper builds considerably on Baumol (1967) seminal paper relying on the supplyside theories of structural change.

${ }^{30}$ See Herrendorf et al. (2013) for a review of estimations on the literature. 
arise from homothetic preferences and then become ${ }^{31}$ :

$$
s_{j}\left(\mathbf{p}_{\mathbf{t}}\right)=\frac{\varphi_{j} p_{j, t}^{1-\varepsilon}}{\sum_{i=g, s} \varphi_{i} p_{i, t}^{1-\varepsilon}}
$$

The simulations we perform in each version of the model follow the same methodology. We set an 'old' stationary equilibrium that represents the economy in the prewar period, in which sectoral technological progress and the economy's long-run technological progress are identical. Then "MIT shocks" on sectoral growth of technology leads to a secular transition path, in which the postwar period is inserted, towards the economy's 'new' stationary equilibrium following assumption $2^{32}$.

It worths comment that the model follows the final consumption expenditure approach as opposed to the value-added one in Herrendorf et al. (2009)'s sense. This means commodities in the utility function are interpreted as the final consumption purchases of the household. Consistency requires that the sectoral production functions be final consumption production functions rather than value-added production functions. We discuss this matter further below.

We follow empirically reasonable choices to most parameters, which means they are based on estimations we carry-out, moments based on these estimations and standard values in either growth or structural change literatures.

\subsection{1 \\ Data and Model Calibration}

We split our model calibration into two tables. Table 13.1 shows the parameters that applies to all three cases with different Stone-Geary specifications. The parameters of intertemporal preference, discount rate $(\rho)$ and the inverse of the intertemporal elasticity of substitution $(\varsigma)$; parameters related to the process of idiosyncratic labor productivity $\left(z_{\text {min }}, z_{\text {max }}, z_{\text {mean }}, \zeta, \sigma^{2}(z)\right)$, the debt limit $(\underline{a})$ and the depeciation rate $(\delta)$ follow Achdou et al. (2017). $\rho$ and $\varsigma$ are standard values. $z_{\text {min }}, z_{\text {max }}, z_{\text {mean }}, \zeta, \sigma^{2}(z)$ are not targeted to inequality moments since this basic version of Bewley-aiyagari model, it is well-known, is not sufficiently rich to be an empirically realistic theory of income and wealth distribution (Achdou et al. (2017); Anh et al., 2017). However, we shall see, this simple description of income and wealth distribution suffices for the model to feature the cross-sectional patterns of sectoral expenditure of households

\footnotetext{
${ }^{31}$ The composite consumption in $(3)$ becomes $\hat{c_{t}} \equiv\left[\sum_{j \in J} \varphi_{j}^{\frac{1}{\varepsilon}}\left(\hat{c}_{j, t}\right)^{\frac{\varepsilon-1}{\varepsilon}}\right]$ and then the following Marshallian demands: $c_{j}\left(\mathbf{p}_{\mathbf{t}}, \hat{e}\left(\mathbf{p}_{\mathbf{t}}, \bar{u}_{t}\right)\right)=\left(\frac{\varphi_{j}}{p_{j, t}^{\varepsilon} \sum_{i=g, s} \varphi_{i} p_{i, t}^{1-\varepsilon}}\right) \hat{e}_{t}$. Composite consumption price follows the baseline model's definition in (12).

${ }^{32}$ We solve the model backwards following Achdou et al. (2017) .
} 
Chapter 2. Reconciling Kuznets Facts, Engel's Law and Relative Prices trend in the postwar US in a Bewley-Aiyagari Framework with Stone-Geary Preferences

grouped by income quintiles. $\underline{a}$ also is not target to any moment in the model and represents around 0.15 of the natural debt limit in 1947. $\delta$ is equivalent to the annual average between 1950-2010 in Penn World Table (Feenstra et al. $(2015))$.

\begin{tabular}{|c|c|c|c|}
\hline Parameter & Value & Source - Target & Comments \\
\hline$\rho$ & 0.035 & Achdou et al. (2017) & Standard value \\
\hline$\gamma$ & 2 & Achdou et al. (2017) & Standard value \\
\hline $\mathrm{z}_{\min }$ & 0.5 & Achdou et al. (2017) & Model scale. To ensure compactness of $\mathrm{Z}$ set. \\
\hline $\mathrm{z}_{\max }$ & 1.5 & Achdou et al. (2017) & Model scale. To ensure compactness of $Z$ set. \\
\hline $\mathrm{z}_{\text {mean }}$ & 1 & Achdou et al. (2017) & $\begin{array}{l}\text { Mean of the Ornstein-Uhlenbeck process (in levels). This } \\
\text { parameter has to be adjusted to ensure that the mean of } z \\
\text { (truncated Gaussian) is } 1 \text {. }\end{array}$ \\
\hline$\zeta$ & 0.3 & Achdou et al. (2017) & $\begin{array}{l}\text { Model scale. It implies a less persistent z process than commonly } \\
\text { estimated by AR(1) processes. See Guvenen (2009). }\end{array}$ \\
\hline$\sigma^{2}(z)$ & 0.01 & Achdou et al. (2017) & Model scale. It captures transitory innovations in z. \\
\hline$\underline{a}$ & -1 & Achdou et al. (2017) & -1 equals $0.15^{*} \mathbb{d}$ in 1947 ; $\mathbb{A}$ is the natural debt limit. \\
\hline$\alpha$ & 0.36 & Herrendorf and Valentinyi (2008) & $\begin{array}{l}\text { Estimated capital income share in total consumption sector } \\
\text { (agriculture, manufacturing and services). Final-consumption } \\
\text { production functions } \\
\text { rather than value-added production functions. }\end{array}$ \\
\hline$\delta$ & 0.05 & Achdou et al. (2017) & $\begin{array}{l}\text { Equivalent to 1950-2010 annual average in Penn World Table - } \\
\text { Feenstra et al. (2015) }\end{array}$ \\
\hline$\psi_{\alpha}$ & 0.02 & Jones (2015) & $\begin{array}{l}\text { Long-run growth rate: average annual growth rate of GDP per } \\
\text { person in the U.S. - 1870-2014 }\end{array}$ \\
\hline$\tau, T$ & $2010 ; 2139$ & 1947-2010 period; Secular transition (192 years) & Arbitrary \\
\hline
\end{tabular}

Table 2.1: Model Calibration - First Part

Notes: This table reports the model calibration for the parameters of intratemporal preference, discount rate $(\rho)$ and the inverse of the intertemporal elasticity of substitution $(\varsigma)$; parameters related to the process of idiosyncratic labor productivity, mean and bounds of labor productivity, the rate of mean reversion and the diffusion, respectively $\left(z_{\text {min }}, z_{\text {max }}, z_{\text {mean }}, \zeta, \sigma^{2}(z)\right)$; the debt limit $(\underline{a})$, the capital share $(\alpha)$, the economy's long-run rate of technological progress $\left(\psi_{d}\right)$, the depeciation rate $(\delta)$ and time periods for the transition. Sources or targets and additional comments are provided for each parameter.

The economy's long-run rate of technological progress $\left(\psi_{d}\right)$ follows Jones (2015), which shows that since 1870 the annual growth rate of GDP per person in the US is considerably steady at 2 percent. Targeting a secular transition, we set $\tau=64$ years to represent the postwar period between 1947-2010 and $T=3 \tau=192$ years. Note that setting any $T>\tau$, even though arbitrarily, it is only a convenience to solve the model backward and it does not change the model results during the postwar period that we aim to illustrate since the growth rates of sectoral technological progress are constant during the targeted periods ${ }^{33}$. For the capital share $(\alpha)$ we follow ÃAkos Valentinyi and Herrendorf (2008) . Consistently with our final expenditure approach, they estimate it in final expenditure production functions to overall consumption

${ }^{33}$ See assumption 2 . We vary $T$ equals to $2 \tau$ and $4 \tau$ to confirm it. 
Chapter 2. Reconciling Kuznets Facts, Engel's Law and Relative Prices trend in the postwar

(agriculture, manufacturing and services) taking into account the the entire input-output structure of the economy for the US in 1997.

Table 22.2 exhibits parameters that vary by each model case of structural change. The initial level of sectoral labor-aumenting productivity, $A_{m, t_{0}}, m=$ $\{d, g, s\}$, sectoral technological progress, $\psi_{j, t}, j=\{g, s\}$ and parameters related to the intratemporal Stone-Geary preferences: the parameter that governs intratemporal elasticity of substitution, $\varepsilon$, the nonhomotheticity term of goods in the 'old' stationary equilibrium, which represents $1947, \bar{c}_{g, 1947}$ and the preference weights of goods and services, $\varphi_{g}$ and $\varphi_{s}$. Regarding parameters of technology, we highlight the following comments. As we mentioned in the beginning of this section, we follow the final expenditure approach. It means that commodities in the utility function are interpreted as the final consumption purchases of the household, which then requires, for consistency, that the sectoral production functions be final consumption production functions rather than value-added production functions ${ }^{34}$. In our case, calibrating exogenous variables and parameters related to technology turns out to be changelling since sectoral production functions must be final consumption production functions. The exact production processes with intermediate inputs have to be specified considering the entire input-output structure in the economy so as to set sectoral real expenditures and associated factors levels ${ }^{35}$, factor shares, labor-aumenting productivities and technological progress in all periods. To assess it in detail is not in the core of our study and it deserves further investigation (see e.g.ÃAzkos Valentinyi and Herrendorf (2008)). For this reason, we leave both initial level of sectoral labor-aumenting productivity and sectoral technological progress free to match aggregate sectoral expenditure shares in initial year, 1947, in all cases (1), (2) and (3) so as they depart from the same levels of sectoral expenditure shares. It implies, since all cases also feature the same long-run rate of technological progress, that they converge to the same level of aggregate capital (and output) in the new stationary equilibrium. Sectoral technological progress are also targeted to match moments of structural change in Table 33.3 in the baseline model (1).

With respect to parameters of the Stone-Geary preferences, we rely on Herrendorf et al. (2009)'s estimation methodology and data from NIPA-BEA ${ }^{36}$.

\footnotetext{
${ }^{34} \mathrm{As}$ an illustration, in our final expenditure approach a meal in a restaurant is a service expenditure both in the utility function and in the services production function, whereas in the value-added approach, a meal in a restaurant represents consumption of food from agriculture, processing from manufacturing and cooking and serving in the restaurant from services.

${ }^{35}$ In section 2, we normalize aggregate labor to 1. Initial aggregate capital follows Achdou et al. (2017).

${ }^{36}$ See appendix D for our application of their estimation methodology.
} 
Chapter 2. Reconciling Kuznets Facts, Engel's Law and Relative Prices trend in the postwar

\begin{tabular}{|c|c|c|c|c|c|}
\hline \multirow[b]{2}{*}{ Parameter } & \multicolumn{3}{|c|}{ Model } & \multirow[b]{2}{*}{ Source - Target } & \multirow[b]{2}{*}{ Comments } \\
\hline & (1) & (2) & (3) & & \\
\hline$A_{d d_{0}}$ & 0.3 & 4.3 & 0.3 & The target is to match aggregate expenditure shares in 1947 & $\begin{array}{l}\text { We set initial level of sectoral labor-aumenting productivity so that } \\
\text { aggregate sectoral expenditures shares are matched with data in the 'old' } \\
\text { stationary equilibrium }\end{array}$ \\
\hline$A_{g \alpha_{0}}$ & 0.5 & 0.28 & 0.2 & The target is to match aggregate expenditure shares in 1947 & $\begin{array}{l}\text { We set initial level of sectoral labor-aumenting productivity so that } \\
\text { aggregate sectoral expenditures shares are matched with data in the 'old' } \\
\text { stationary equilibrium }\end{array}$ \\
\hline$A_{s, t_{0}}$ & 0.58 & 0.58 & 3.3 & The target is to match aggregate expenditure shares in 1947 & $\begin{array}{l}\text { We set initial level of sectoral labor-aumenting productivity so that } \\
\text { aggregate sectoral expenditures shares are matched with data in the 'old' } \\
\text { stationary equilibrium }\end{array}$ \\
\hline$\left\{\psi_{g, t}\right\}_{t=1947}^{2009}$ & 0.037 & 0.02 & 0.037 & The target is to match moments in table 3 & $\begin{array}{c}\text { Models (1) and (3): For } 1947 \leq \mathrm{t}<2010: \psi_{g z}=0.03 \text {. For } 2010 \leq \mathrm{t}<2139: \\
\psi_{g, t} \text { converges smoothly to } \psi_{d, t} \text {. For } \mathrm{t} \geq 2139: \psi_{g t}=\psi_{d s} \\
\text { Model (2): } \psi_{g, t}=\psi_{d,} \text { for all t. }\end{array}$ \\
\hline$\left\{\psi_{\mathrm{s}, \mathrm{t}}\right\}_{\mathrm{t}=1949}^{2009}$ & 0.01 & 0.02 & 0.01 & The target is to match moments in table 3 & $\begin{array}{c}\text { Models (1) and (3): For } 1947 \leq \mathrm{t}<2010: \psi_{s, t}=0.005 \text {. For } 2010 \leq \mathrm{t}<2139 \\
\psi_{s t} \text { converges smoothly to } \psi_{d, s} \text {. For } \mathrm{t} \geq 2139: \psi_{s, t}=\psi_{d, s} \\
\text { Model (2): } \psi_{s, t}=\psi_{d, t} \text { for all t. }\end{array}$ \\
\hline$\varepsilon$ & 0.57 & 1 & 0.57 & $\begin{array}{l}\text { Estimated in NIPA-BEA data from 1947-2010. In model (2) } \\
\text { parameter is imposed by construction }\end{array}$ & Estimations follow Herrendorf et al. (2013). See appendix C. \\
\hline$-\overline{\mathrm{c}}_{\mathrm{g}, 1947}$ & 0.59 & 0.26 & & $\begin{array}{l}\text { Estimated in NIPA-BEA data from 1947-2010. In the baseline } \\
\text { model (1) the target is to match moments in table x }\end{array}$ & $\begin{array}{l}\text { Estimations follow Herrendorf et al. (2013). See appendix C. } \\
\text { For } 1947 \leq t<2010:-\bar{c}_{g, 1947} \text { exp }\left\{\psi_{d} s\right\}, s \geq 1 \text {. For } 2010 \leq t<2139 \text { : coverges } \\
\text { smoothly to }-0.08 \bar{c}_{g, 1947} \text { for } t \geq 2139 \text { in models }(1) \text { and (2) }\end{array}$ \\
\hline$\varphi_{g}$ & 0.43 & 0.43 & 0.43 & Estimated in NIPA-BEA data from 1947-2010 & Estimations follows Herrendorf et al. (2013). See appendix C. \\
\hline$\varphi_{s}$ & 0.57 & 0.57 & 0.57 & Estimated in NIPA-BEA data from 1947-2010 & Estimations follows Herrendorf et al. (2013). See appendix C. \\
\hline
\end{tabular}

Table 2.2: Model Calibration - Second Part

Notes: This table reports the model calibration for the initial level of sectoral labor-aumenting productivity, $A_{m, t_{0}}, m=\{d, g, s\}$, sectoral technological progress, $\psi_{j, t}, j=\{g, s\}$ and parameters related to the intratemporal Stone-Geary preferences: the parameter that governs intratemporal elasticity of substitution, $\varepsilon$, the nonhomotheticity term of goods in the 'old' stationary equilibrium, which represents $1947, \bar{c}_{g, 1947}$ and the preference weights of goods and services, $\varphi_{g}$ and $\varphi_{s}$. Sources or targets and additional comments are provided for each parameter.

However, unlike these authors, since we focus on consistency with household final-expenditure data from CE-BLS to reconcile Kuznets facts with Engel's law, data are subject to smaller consistency issues due to data source differences in the level of aggregation from this two different data sources. It implies, for example, that we exclude government consumption expenditure from NIPA-BEA data ${ }^{37}$. Furthermore, unlike Herrendorf et al. (2009)who estimate the preference parameters in three consumption sectors' shares (agriculture, manufacturing and services), we add manufacturing to agriculture in the goods sector in addition to services to improve the matching with CE-BLS cross-sectional households final-expenditure data. Reported expenditures in the $\mathrm{CE}$ interviews surveys reveal well-known systematic mis-measurements (Aguiar and Bils, 2015) specially related to durable goods in the manufacturing sector. Durable goods differences in expenditure across income quintiles do not necessarily align with differences in durable stocks and associated service flows and therefore it might obliterate cross-sectional differences of consumption patterns that we mean to address related to the Engel's law. For this reason, to

${ }^{37}$ See data appendix $\mathrm{C}$ for more details. 
Chapter 2. Reconciling Kuznets Facts, Engel's Law and Relative Prices trend in the postwar

mitigate these mis-measurements, our Stone-Geary specification features only one broad goods sector and we will see in the results section on Engel's law that we target the cross-sectional nondurable final-expenditure of the households in CE-BLS data. For the estimation in two sectors, we then follow Matsuyama (2016) by imposing $\bar{c}_{s}=0^{38}$ (see the first column of Table A.1 in appendix A for the estimation results of the Stone-Geary preferences parameters in the baseline model (1)).

\begin{tabular}{|c|c|c|c|c|}
\hline & \multicolumn{2}{|c|}{$\begin{array}{l}\text { Estimated Model } \\
\text { partial equilibrium }\end{array}$} & \multicolumn{2}{|c|}{$\begin{array}{l}\text { Calibrated Model } \\
\text { general equilibrium }\end{array}$} \\
\hline & 1947 & 2010 & 1947 & 2010 \\
\hline$-p_{g} \bar{c}_{g} / \hat{E}$ & 0.32 & 0.05 & 0.32 & 0.05 \\
\hline
\end{tabular}

Table 2.3: Targeted Moments - Nonhomotheticity Term of Goods Relative To Final Consumption Expenditure

Notes: This table reports in the first two columns the ratio of the value of the goods subsistence relative to aggregate total expenditure estimated on data from NIPA-BEA that follows Herrendorf et al. (2009)'s partial equilibrium approach and methodology for 1947 and 2010. Data is subject to smaller consistency issues due to data source differences with CE-BLS. The last two columns exhibit the same moments by the calibrated general equilibrium baseline model. See the data appendix for further details on definitions of expenditure sectors.

Finally, as mentioned in Table $22.2, \bar{c}_{g, 1947}$ and $\psi_{j}, j \in\{g, s\}$ were calibrated so that the sequence of $\hat{\bar{c}}_{g, t}$ yields the moments estimated in data by the baseline model (1), which follows the partial equilibrium approach in Herrendorf et al. (2009). (2013). We see in Table 33.3 these targeted moments predicted by our general equilibrium model, where we define $\hat{E}_{t}=$ $\int_{\underline{z}}^{\bar{z}} \int_{\underline{a}}^{\infty} \hat{e_{t}} \mathfrak{g}(\hat{a}, z, t) d a d z$ as aggregate total expenditure.

\footnotetext{
${ }^{38}$ Note that although we do not consider services endownments through $\bar{c}_{s}$, it is only necessary one nonhomotheticity term to be different from zero (in our case interpreted as food subsistence term within the goods sector, $\bar{c}_{g}$ ) for the two-sectors to feature income effects of the structural change in both sectors. We also estimate the preference parameters in three consumption sectors (agriculture, manufacturing and services). It turns out that the nonhomotheticity term of agriculture is rather similar to the one of the goods sector and the preference weights of agriculture and manufacturing also add up similarly to the goods sector's preference weight. Therefore, either specifying two or three consumption sectors does not considerably affect the fit of the model.
} 


\subsection{2}

Results

In the results section we present how the model performs in matching the Kuznets facts, which is the relative rise of the aggregate expenditure share of services, and the what it implies for the other two main stylized facts of structural change in the postwar US: the Engel's law, or the cross-sectional pattern of sectoral expenditure of households grouped by income quintiles and the decreasing trend of the relative price of goods. Before that, in the beggining of this section, we briefly discuss on how our two-sector Bewley-Aiyagari model shapes the intertemporal opportunities and, in turn, how it qualitatively affects sectoral dynamics.

\subsubsection{1}

\section{Intertemporal opportunities and sectoral dynamics}

In Herrendorf et al. (2009), the authors carry out a partial equilibrium approach focused on the households intratemporal problem. Accordingly, they do not take a stand on the exact nature of intertemporal opportunities available to the household and on how expectations of the future are formed. In our framework, instead, we do so. Expectations are rational, which in our environment means that in the beginning of the transition households know the economy will start a growth path towards a richer economy in the new stationary equilibrium. Households also know all parameters, the technological shocks the economy faces and therefore know the prices trajectory. This is key in our model, since we will show that the two main driving forces of structural change, namely, the paths of sectoral technological progress (relative prices) and the nonhomotheticity term of goods subsistence (income effects), also affect intertemporal opportunities.

Note that, since in the transition beginning households know they will become richer, there is a 'natural' wish to smooth consumption expenditure arising from intertemporal preferences. In addition, we mentioned in previous section that we calibrate each case $(1),(2)$ and (3), so that they converge to the same level o capital (and output) in the new stationary equilibrium. Thus, households know in the transition beginning that the economy will feature a lower (and equivalent in all model cases) level of interest rate. The Euler equation $^{39}$ synthesizes the intuition on how $r_{t}$ interacts with intertemporal preferences in the wish to smooth consumption expenditure.

$$
\frac{\mathbb{E}_{t}\left\{d \nu^{\prime}(e(\hat{a}, z, t))\right\}}{\nu^{\prime}(e(\hat{a}, z, t))}=\left(\hat{\rho}+\psi_{d}-r_{t}\right) d t
$$

\footnotetext{
${ }^{39}$ See appendix B for the derivation of the Euler equation.
} 
Chapter 2. Reconciling Kuznets Facts, Engel's Law and Relative Prices trend in the postwar

Therefore, for given $\hat{\rho}$ and $\psi_{d}$, the path of interest rate througout the transition towards a richer economy implies that the expenditure-savings tradeoff is smaller in the transition beginning. Hence, it turns out to be optimal to anticipate consumption expenditure (and to decumulate assets) - see panels $\mathrm{A}, \mathrm{B}$ and $\mathrm{C}$ in figure 3 .
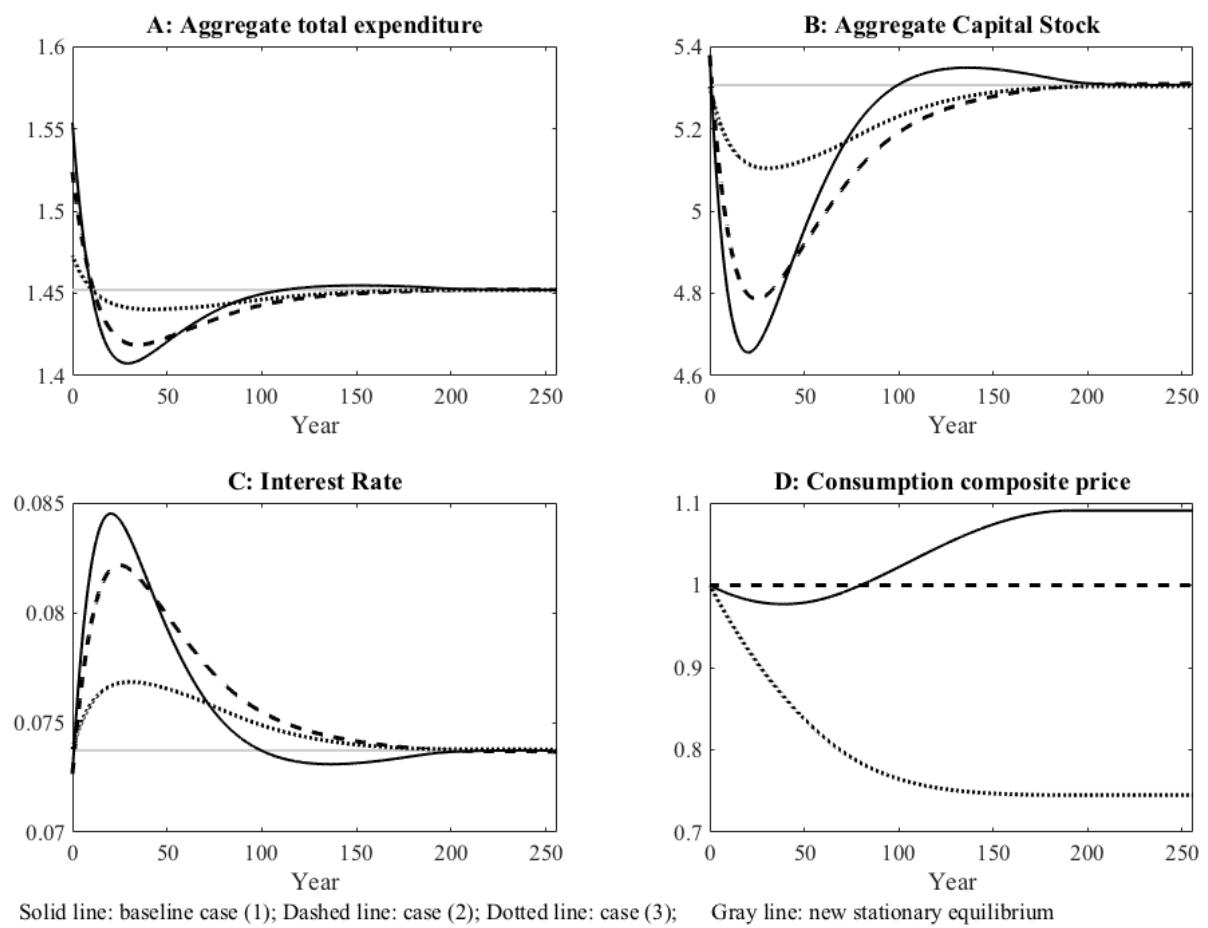

Solid line: baseline case (1); Dashed line: case (2); Dotted line: case (3);

Gray line: new stationary equilibriun

Figure 2.3: Transition Dynamics of Aggregate Total Expenditure, Aggregate Capital Stock, Interest Rate and composite consumption Price

Notes: This figure depicts the transition dynamics for aggregate total expenditure, aggregate capital stok, interest rate and composite consumption price, in panels A, B, C and D, respectively. Black solid lines: baseline case (1), dashed lines: case (2) and dotted lines: case (3). Gray lines represent levels in the new stationary equilibrium. The time range in the transition between the old stationary equilibrium and the new stationary equilibrium is 256 years. See assumption 2 and section 3.1 for details on targeting the secular transition.

By looking at panel $\mathrm{A}$ of figure 3, in particular, one might ask what distinguishes the level of consumption expenditure anticipation in the transition beggining in each case. The answer is related to sectoral dynamics in our two-sector environment. The composite consumption price ${ }^{40}, p_{t}$, and the nonhomotheticity term, $\bar{c}_{g}$, also affect intertemporal opportunities expressed by the Euler equation (31) since they affect expenditure (and savings) policy functions. We also see from (12) that the composite consumption price is a

\footnotetext{
${ }^{40}$ Remind from the baseline model setup in the technology section, we normalize investment prices so that $p_{t}$ represents the rate in which the economy transforms consumption and capital - the marginal rate of transformation.
} 
composition of sectoral prices $^{41}, p_{j, t}, j=\{g, s\}$. Hence, the paths of sectoral prices stemming from different assumptions both with respect to the growth rate of sectoral technological progress and also related to the nonhomotheticity term in each case will affect the intensity of the wish to anticipate consumption expenditure. Rewriting equation (22), which defines the savings policy (and expenditure policy), and total expenditure definition helps in clarifying this intuition:

$$
y(\hat{a}, z, t)=\hat{w}\left(Q_{t}\right) z_{t}+\left(r\left(Q_{t}\right)-\psi_{d}\right) \hat{a}_{t}-e(\hat{a}, z, t),
$$

where it is well-known that $e(\hat{a}, z, t)=p_{t} c_{t}-p_{g, t} \bar{c}_{g}$, such that $\bar{c}_{g}<0$ according to the estimation results in table A.1 in appendix A.

Panel D of figure 3 complements equation (22) and expenditure definition in grasping this intuition. It exhibits the composite consumption price. First remind, as panel B illustrates, we keep the level of capital (and output) the same in all cases in the new stationary equilibrium ${ }^{42}$. Therefore, apart from differences with respect to assumptions of each case and how they affect the Euler equation, the wish to smooth consumption expenditure due to the transition towards a richer economy should be the same in all three cases. In case (2) the composite consumption price is flat by assumption, so that we turn the price effects off in comparison to baseline case (1). Since $p_{t}$ is flat and in a lower level, it strenghens the expenditure-savings trade-off in the transition beginning relative to the baseline case. That is, by following $e(\hat{a}, z, t)$ definition, expenditure anticipation is slightly lower and then aggregate capital reduces relatively less in the transition beginning (it weakens the households' wish to decumulate assets) ${ }^{43}$, as we see in panels A and B of figure 3.

In case (3), expenditure anticipation is the lowest and then aggregate capital feature the smallest declining in the transition beginning, according to panels A and B in figure 3. Since composite consumption price declines and reaches the lowest level relative to the other cases in the new stationary

\footnotetext{
${ }^{41}$ Even though equation (12) defines the composite consumption price in the baseline case (1), it does not invalidate the generality of the argument that the composite consumption price is also a composition of sectoral prices. See footnotes 26 and 29 for specifications of composite consumption and its price in cases (1) and (2), respectively.

${ }^{42}$ It this imples in panels $\mathrm{A}$ and $\mathrm{C}$ that aggregate expenditure and interest rate also converge in the new stationary equilibrium.

${ }^{43}$ Moreover, since composite consumption is relatively cheaper than in the baseline case and we impose the same level of aggregate capital and output in the new stationary equilibrium, the level of composite consumption must be higher than the in the baseline case. To see this from the aggregate output perspective, let's consider market clearing conditions (16) and aggregate total expenditure definition. Thus, we have that $Y_{t}=\sum_{j} p_{j, t} C_{j, t}+D_{t}=$ $E_{t}+D_{t}=p_{t} C_{t}+p_{g, t} \bar{c}_{g}+D_{t}$, where it is clear that, all else unchanged, if $p_{t}$ is lower, $C_{t}$ must be higher than the baseline case. See figure A.1 in appendix A.
} 
equilibrium, the expenditure-savings trade-off in the transition beginning is the strongest one. In addition, considering definition of $e(\hat{a}, z, t)$, once we turn the nonhometheticity term off, expenditure anticipation should be indeed the lowest one relative to the other cases.
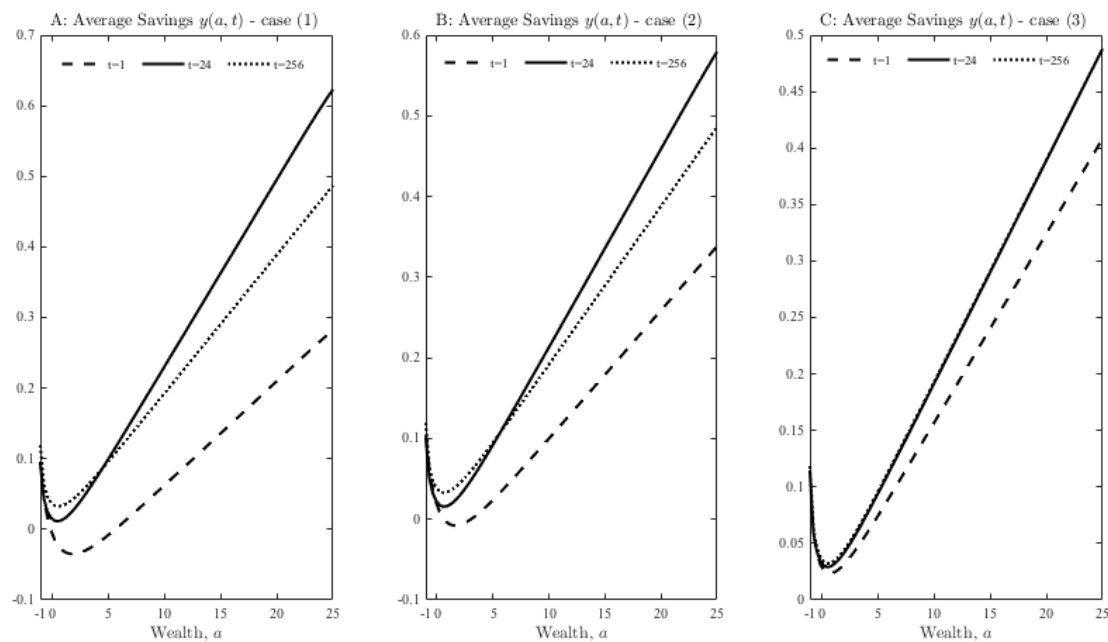

Figure 2.4: Average Savings in Selected Periods of the Transition Dynamics

Notes: This figure depicts the average savings $y(a, t) \equiv \int_{Z} y(a, z, t) d z$ over the grid of wealth, $a$, for $t=1$, the transition beginning; $t=24$, when interest rate peaks; and $t=256$, the new stationary equilibrium for the baseline model case (1), case (2) and case (3) in panels A, B and C, respectively.

Finally, as households dwindle assets and aggregate capital declines in the transition beginning, interest rate rises, as illustrates panel $\mathrm{C}$ of figure 3 . As interest rate rises, the average savings becomes more positively steep with respect to asset holdings, $a$, as shows equation $(22)^{44}$. When interest rate is sufficiently high, at its peak, it means the richest households save relatively more so that aggregate capital reverts and starts to increase towards the new stationary equilibrium. Figure 4 plots the savings policy in the three cases in the first year of the transition, $t=1$, when $r_{t}$ reaches its peak in $t=24$ and in the new stationary equilibrium in $t=256$. We see that it is more pronounced in baseline case (1) in panel A, followed by cases (2) and (3) due to the interest rate paths in these cases. The theorethical support behind the shape of the savings function follows Jensen (2017), which generalizes Carroll and Kimball (1996)'s result on concave consumption functions (convex savings functions) to the Aiyagari (1994) setting with borrowing constraints in a framework rather

\footnotetext{
${ }^{44}$ Average savings here, $y(a)$, means we integrate $y(a, z)$ over $z$. We could show the savings policy over $(a, z)$. However, since it is virtually monotonically increasing in $z$, for easy of presentation we plot $y(a)$, otherwise it would be more subtle to visualize the savings policy becoming more positively steep in $a$. See the saving plots over $(a, z)$ in figures A.2-A.4 in appendix A for the baseline case (1), case (2) and case (3), respectively.
} 
Chapter 2. Reconciling Kuznets Facts, Engel's Law and Relative Prices trend in the postwar

similar to this study ${ }^{45}$. Moreover, note that the model generates a contingent of restricted (hand-to-mouth) households (households that hold zero or are endebted with negative assets) ${ }^{46}$ whose savings policy has a negative slope ${ }^{47}$.

\subsubsection{2}

\section{Fact i: Relative price of goods}

As we highlight in figure 2, the price of goods relative to services declines over the postwar period. We mentioned that the two data sources we use for final-consumption expenditure and prices in this analysis (BEA and BLS) are internally consistent. Both expenditure and price data from BEA are drawn from NIPA tables and are focused to depict the relative consumer price between goods and services and the Kuznets facts. Final-consumption expenditure from BLS is drawn from the CE interviews and are focused to depict the relative consumer price between goods and services and the Engel's law from the 80 's. Sectoral definitions in expenditure data from both sources are subject to consistency issues to maximize the match in the levels of sectoral aggregate shares, as illustrates figure 1. In order to make price data also be consistent, we also define sectoral consumer price from CPI-BLS accordingly to sectors in CE-BLS final-consumption data. Figure 2 also shows that it then implies a rather reasonable match of the price of goods relative to services drawn both from NIPA-BEA and from CPI-BLS ${ }^{48}$.

It worth noting that in calibrating the baseline model we have not targeted sectoral price dynamics. Instead, relative prices dynamics stem from sectoral technological progress, which are set exogenously and targeted, in turn, to moments of structural change in table 3 3.3. Having mentioned it, we see in figure 5 that the baseline case (1) does a reasonable job of capturing the declining long-run trend of relative price of goods data from NIPA-BEA ${ }^{49}$

${ }^{45}$ The main difference with Jensen (2017)'s environment is that time is discrete in the author's study, which does not affect this result. Furthermore, although the author applies a canonical one-sector Aiyagari model, our model behaves exactly as a canonical one-sector model in the intertemporal problem. The similarity specially refers to preferences in HARA (CRRA) family, which is key for this result. See theorem theorem 4 in Jensen (2017).

${ }^{46}$ Note that one-asset heterogeneous agent models in the spirit of Aiyagari (1994) are illsuited to match the fraction of hand-to-mouth households (Ahn et al., 2017; Kaplan et al., 2018).

${ }^{47}$ Our case is similar to proposition 1 in Achdou et al. (2017), which applies a Poisson productivity process instead of an Ornstein-Uhlenbeck diffusion process of productivity in our case. It deserves further investigation, which is not in the core of this paper, but there seems to exist $0<a_{\min }^{z}$ such that $y(a, z)<0$ for any $\underline{a}<a \leq a_{\min }^{z}$ and for any $z<z_{\min }$, such that $z_{\min } \in[\underline{z}, \bar{z}]$. See figures A.2-A.4 in appendix A with $y(a, z)$. One can also see the densities in figure A.5 in appendix A.

${ }^{48}$ See data appendix for details on data sources. Particularly for the consistency between CE-BLS sectoral expenditure data and CPI-BLS sectoral price data, see the first chapter of this thesis.

${ }^{49}$ Note that price data is filtered using Hodrick-Prescott filter 

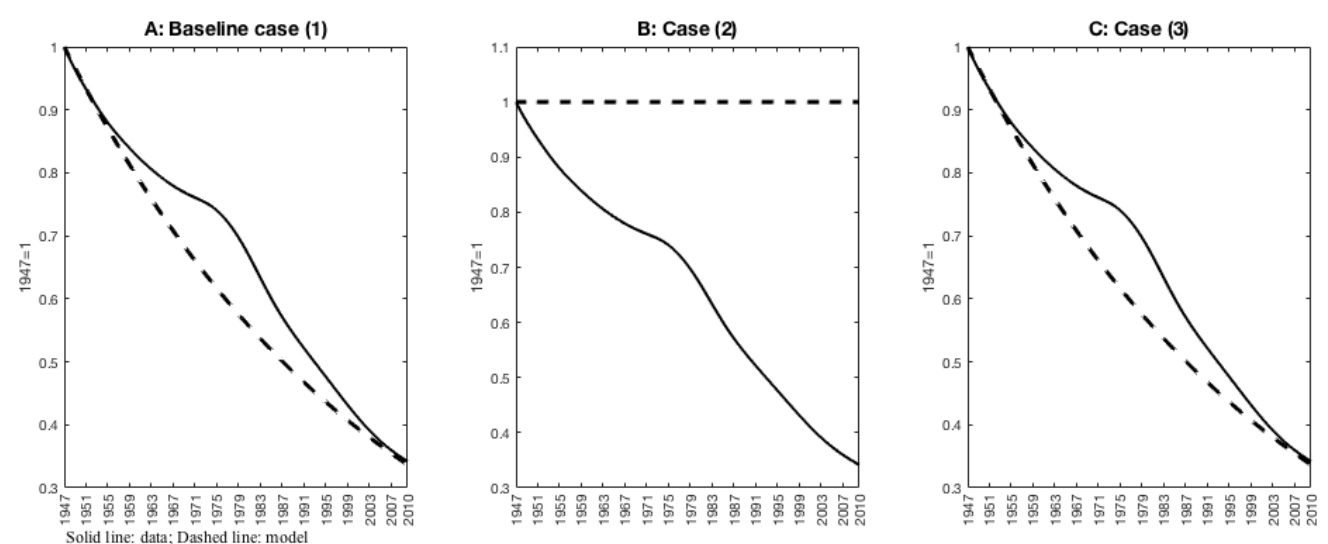

Figure 2.5: Fit of the Model to Goods to Services Prices Ratio in the Postwar US

Notes: This figure depicts the fit of the model to the ratio of goods to services prices using data from NIPA-BEA for goods (agriculture and manufacturing) and services. Data follows Herrendorf et al. (2009) subject to smaller consistency issues due to data source differences with expenditure data from CE-BLS and price data from CPI-BLS. Definition of sectors and its components with data from CPI-BLS matches to sectoral definitions with data from CE-BLS. Solid and dashed lines denote, respectively, data from NIPABEA (smoothed with Hodrick-Prescott filter) and predictions of the model cases (1), (2), (3) in panels A, B and $\mathrm{C}$. See the data appendix for further details on definitions of expenditure sectors.

in the postwar period. In case (3), the only difference relative to case (1) is that we turn the nonhomotheticity term off, keeping all else unchanged. Since by relative prices definition in (11), it only depends on sectoral technological progress and they are the same in cases (1) and (2), hence naturally both cases feature identical trajectories of relative price of goods. Case (2) cannot feature this emprical regularity by assumption. Conversely to case (3), we turn the price effects off so that sectoral technological progress are identical to long-run technological progress of the economy. Thus, relative prices dynamics is flat.

\subsubsection{3}

\section{Fact ii: Kuznets facts}

In terms of the empirical regularity on a falling aggregate expenditure share of goods and a rising aggregate expenditure share of services in the postwar period - Kuznets facts -, our calibration strategy succeeded in featuring a good fit of the model to the data on final consumption expenditure shares in the baseline case (1) when we do not impose any restrictions on the parameters, according to panel A of figure 6 .

Cases (2) and (3) represent a way to judge the importance of income versus relative prices. First note that in the baseline case (1) the nonhomotheticity term is sizable, standing for 0.50 and 0.13 of the aggregate consumption quantities of goods, $\hat{\bar{c}}_{g} \hat{C}_{g, t}$, respectively in 1947 and 2010, which suggests that income effects could play an important role in shaping the shares of final con- 

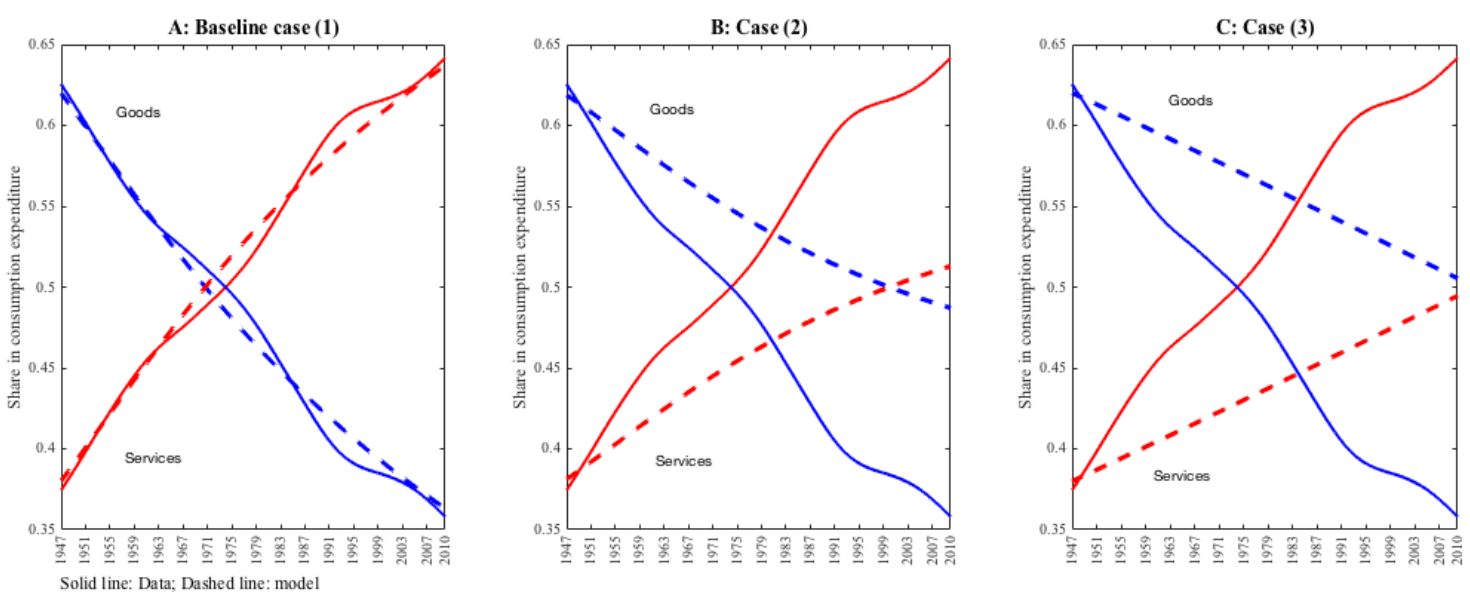

Figure 2.6: Fit of the Model to Kuznets Facts in the Postwar US

Notes: This figure depicts the fit of the model to the aggregate sectoral shares of consumption expenditure - Kuznets facts - using data from NIPA-BEA in 1947-2010 (smoothed with Hodrick-Prescott filter) for goods (agriculture and manufacturing) and services following Herrendorf et al. (2009) subject to smaller consistency issues due to data source differences. Solid and dashed lines denote, respectively, data from NIPA-BEA and the model cases (1), (2), (3) in panels A, B and C, respectively. See the data appendix for further details on definitions of expenditure sectors.

sumption expenditure.

In case (2), price effects are turned off by imposing $\varepsilon=1$ and that $\psi_{d}=\psi_{j}, j=\{g, s\}$. In case $(1), \varepsilon=0.57$. Thus, a higher $\varepsilon$ implies that households respond to the given decrease in the relative price of goods by substituting away from services, which provide an intuiton behind the lack of structural change on the aggregate shares of consumption expenditure in case (2), as shows panel B of figure 6. Alternatively, remind of expenditures shares definition in this case in (29). We see that the aggregate expenditure share of services is bounded above, since it assymptotically converges to the sector preferences weight, $\varphi_{s} \equiv\left(1-\varphi_{g}\right)=0.57$ as aggregate total expenditure increases indefinitely ${ }^{50}$. Since structural change occurs at a much faster pace, case (2) is not able to feature a good fit to data, which suggests price effects are indeed a relevant force of structural change.

In case (3) exhibited in panel $\mathrm{C}$ of figure 6 , the fit slightly deteriorates relative to case (2). Remind by (30) that sectoral expenditure shares in this case are homothetic, so that we turned off income effects $\left(\bar{c}_{g}=0\right)$. Overall, we conclude that price effects associated to sectoral technological progress and income effects associated with the nonhomotheticities are of virtually equivalent importance as sources of the observed structural change in the shares of final consumption expenditure in the postwar US. This findings

${ }^{50}$ According to $(29), S_{s}\left(\mathbf{p}_{\mathbf{t}}, \hat{E}_{t}\right)=\varphi_{s}\left(1-\frac{p_{g, t} \hat{\hat{c}}_{g}}{\hat{E}_{t}}\right)$, such that capital letters $S$ and $\hat{E}_{t}$ stand for aggregate expenditure share and aggregate total expenditure, respectively. Hence, $S_{s}\left(\mathbf{p}_{\mathbf{t}}, \hat{E}_{t}\right) \stackrel{\hat{E}_{t} \rightarrow+\infty}{\rightarrow} \varphi_{s}$. 
contrasts with previous studies (Herrendorf et al. (2009) , 2013 and Comin et al., 2015) that attribute to income effects the bulk of the structural change. It occurs for different reasons. In Herrendorf et al. (2009), which also apply Stone-Geary preferences, it follows due to the differences in the level of sectoral aggregation. Herrendorf et al. (2009) show that by excluding government consumption from the services sector, which is our case since we aim at approximating expenditure series from NIPA-BEA to expenditure series from CE-BLS, it lowers the estimated term of nonhomotheticity. It then implies that sectoral expenditure shares becomes less elastic to total expenditure and then it increases the explanatory power of relative prices to structural change. Comin et al. (2015) apply nonhomothetic CES preferences, which in turn, implies non-vanishing income effects in contrast to Stone-Geary preferences, which are hardwired to feature transitory income effects. On the other hand, our result on this matter is consistent with the finds in Boppart (2014) also applied to postwar US, although it stems from different reasons. In our case, the income effects vanish asymptotically by the Stone-Geary specification. Boppart 's demand system based on price-independent generalized-linear (PIGL) preferences implies that the price elasticity of services relative to the rest of consumption is declining as the economy grows. As noted by Buera and Kaboski (2009), since the relative expenditure of services grows at a faster rate than services relative price, a declining price elasticity automatically increases the explanatory power of relative prices to structural change.

\subsubsection{4}

\section{Fact iii: Engel's law}

We see in figure 1, which motivates this study in the introduction, that poor (rich) households in the bottom (top) income quintiles spend a larger fraction of their total expenditure on goods (services) than do rich (poor) households in the US, which we name (generalized) Engel's law following Matsuyama (2019) . This empirical regularity seems quite general since Boppart (2014) shows it also applies to other developed OECD countries - and we add an emerging country evidence in Brazil in figure A.6 in appendix A.

Figure 7 illustrates in panel A, B and C how cases (1), (2) and (3) fulfill Engel's law, respectively. We confirm by construction that Engel's law calls for a theory with nonhomothetic preferences since, unlike cases (1) and (2), case (3) has homothetic expenditure shares. Since only price effects drive structural change in this case, all households feature the same sectoral expenditure share at a period in time regardless of its income and total expenditure levels. Note 
that Engel's law in case (2) is in accordance with aggregate levels of sectoral expenditure shares presented in previous section on Kuznets facts.
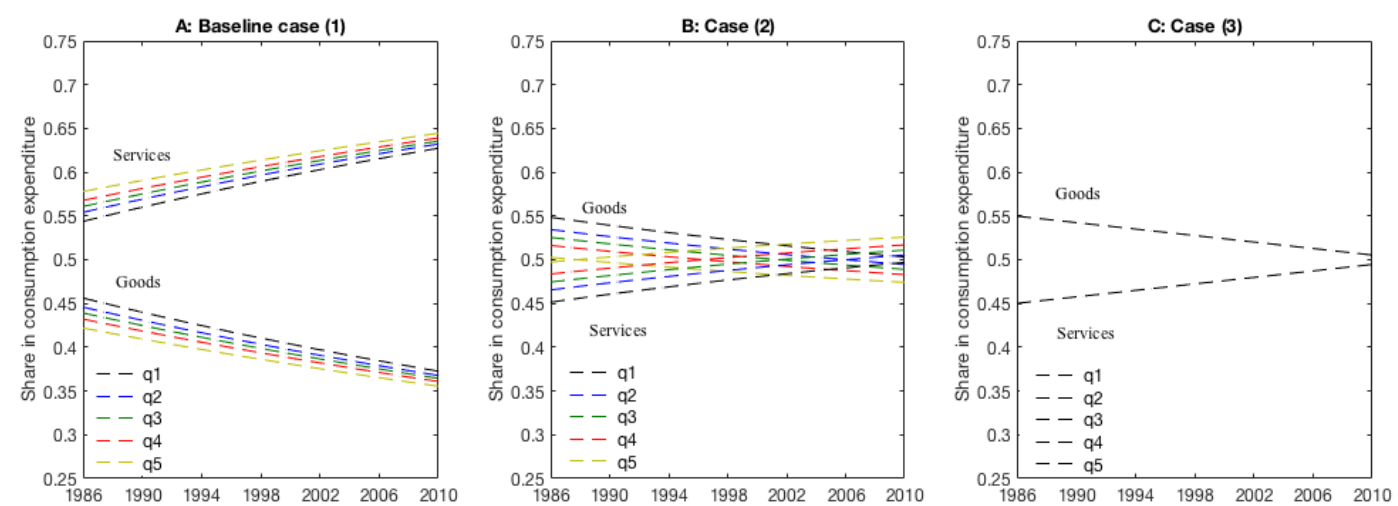

Figure 2.7: Engel's Law in the in the US - Predictions of the Model - 19862010

Notes: This figure depicts the sectoral shares of households grouped by income quintiles -Engel's Law - predicted by the model cases (1), (2) and (3) in panels A, B and C, respectively. See the data appendix for further details on definitions of expenditure sectors.

To reconcile Kuznets facts and Engel's law by bringing the model to data raises some challenges. First, as we mentioned before, aggregate evidence on Kuznets facts and micro evidence of expenditure shares (Engel's law) have different data sources (NIPA-BEA and CE-BLS, respectively), which demands consistency issues due to data source differences in the level of aggregation of household final-expenditure. Second, micro data is only available from the 80's, which narrows our range period of analysis. Moreover, reported expenditures in the CE interviews surveys reveal well-known systematic mis-measurements (Aguiar and Bils, 2015) specially related to durable goods in the manufacturing sector. Durable goods differences in expenditure across income quintiles do not necessarily align with differences in durable stocks and associated service flows and therefore it might obliterate cross-sectional differences of consumption patterns that we mean to address synthesized by the Engel's law (see figure 8). For this reason, to mitigate these mis-measurements, our Stone-Geary specification features only one broad goods sector (instead of splitting it into agriculture and manufacturing ${ }^{51}$ ) and in the baseline case (1) we will target the cross-sectional nondurable final-expenditure of the households in CE-BLS data, since Engel's law shows up more clearly. For this reason we propose to extend the baseline model so that the goods commodities become a composite of durables and nondurables.

${ }^{51}$ This consumption pattern by income quintiles in the manufacturing sector becomes quite confusing specially due to mis-measurements related to durables consumption. 


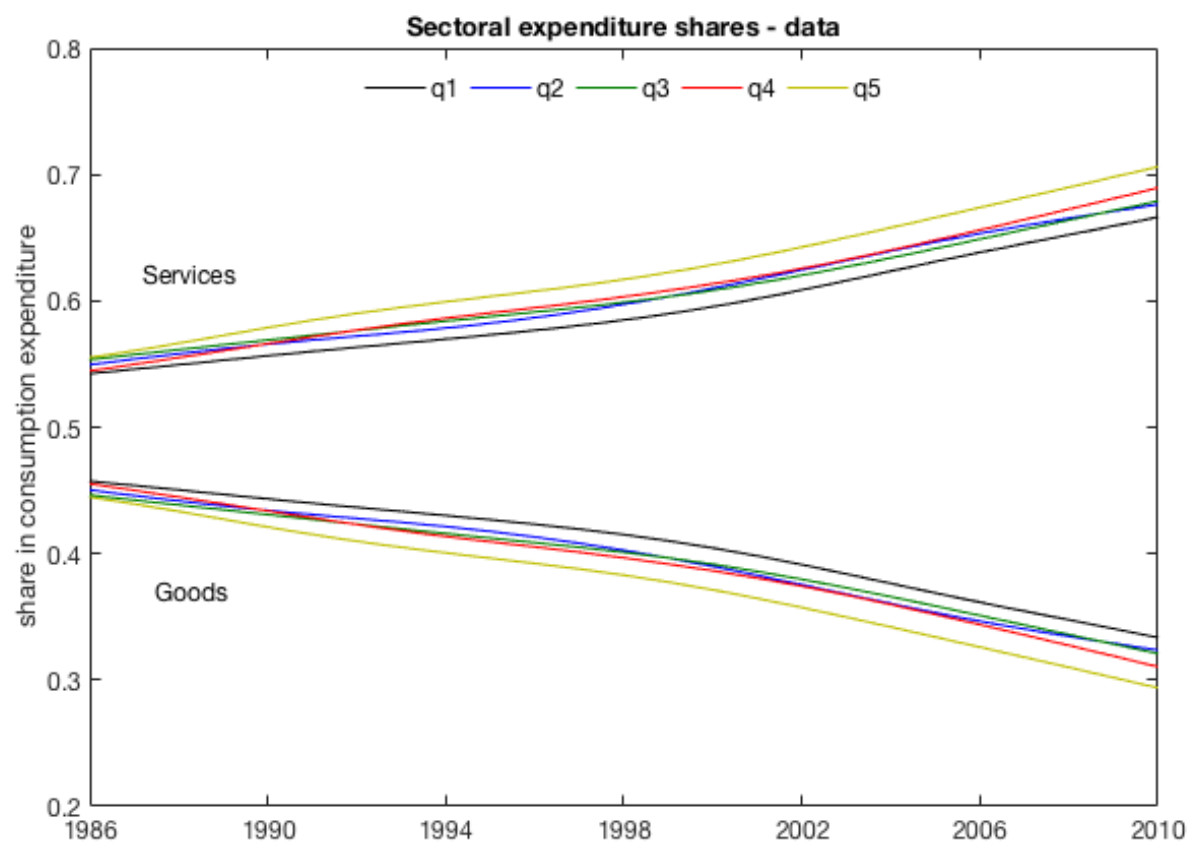

Figure 2.8: Engel's Law in the in the US According to Reported Expenditure in CE-BLS -1986-2010

Notes: This figure depicts the sectoral shares of households grouped by income quintiles with data from reported expenditures in the interviews of the CE-BLS for goods (agriculture and manufacturing) and services. Aggregate data on sectoral expenditure sahres from CE-BEA approximates in levels a measure of household sectoral expenditure shares with data from NIPA-BEA. See the data appendix for further details on definitions of expenditure sectors.

\subsubsection{4}

\section{Extending the baseline model to improve the fit of the Engel's law to data}

To improve the model fit we focus on nondurable consumption as an attempt to mitigate mis-measurements arising specially from durables in the CE-BLS data. Thus, we propose to extend the model so that the goods commodities become a composite of durable and nondurable goods. That is, the broad goods sector is split into durables and nondurables. We only highlight below the main changes implied by this extension of the baseline model. The reminder of the model remains exactly the same as the model set up presented in section 2. Let's start by the intratemporal household problem.

In the intratemporal problem, households now allocate consumption expenditure in $t$ among three types of consumption commodities, $\hat{c}_{f, t}$, where $f \in F=\left\{g_{d}, g_{n d}, s\right\}$, such that indexes $g_{d}$ and $g_{n d}$ stand for durable goods and nondurable goods, respectively: 


$$
\min _{\left\{\hat{c}_{f, t}\right\}_{f \in F}} \sum_{f \in F} p_{f, t} \hat{c}_{f, t} \quad \text { s.t }
$$

$u\left(\hat{c}_{g, t}, \hat{c}_{s, t}\right) \geq \tilde{u}\left(\mathbf{p}_{\mathbf{t}}, e\left(\mathbf{p}_{\mathbf{t}}, \bar{u}_{t}\right)\right) \equiv\left[\varphi_{g}^{\frac{1}{\varepsilon}}\left(\hat{c}_{g, t}+\hat{\bar{c}}_{g}\right)^{\frac{\varepsilon-1}{\varepsilon}}+\left(1-\varphi_{g}\right)^{\frac{1}{\varepsilon}}\left(\hat{c}_{s, t}\right)^{\frac{\varepsilon-1}{\varepsilon}}\right]^{\frac{\varepsilon}{\varepsilon-1}}$

where $\left[\varphi^{\frac{1}{\varepsilon}}\left(\hat{c}_{g, t}+\hat{\bar{c}}_{g}\right)^{\frac{\varepsilon-1}{\varepsilon}}+\left(1-\varphi_{g}\right)^{\frac{1}{\varepsilon}}\left(\hat{c}_{s, t}\right)^{\frac{\varepsilon-1}{\varepsilon}}\right]^{\frac{\varepsilon}{\varepsilon-1}} \equiv \hat{c}_{t}$ is the same Stone-Geary composite consumption as in problem $(3)^{52}$, except for the following additional restriction:

$$
\hat{c}_{g, t}=\left[\phi_{g_{d}}\left(\hat{c}_{g_{d}, t}\right)^{\frac{\gamma-1}{\gamma}}+\left(1-\phi_{g_{d}}\right)\left(\hat{c}_{g_{n d}, t}+\hat{\bar{c}}_{g_{n d}}\right)^{\frac{\gamma-1}{\gamma}}\right]^{\frac{\gamma}{\gamma-1}},
$$

where $\phi_{g_{d}} \leq 1$ is a preference weight on durable goods, $\gamma$ is the parameter that governs the within-period elasticity of substitution between durables and nondurables and $\hat{\bar{c}}_{g_{n d}}$ is the nonhomotheticity term of nondurables. This Stonegeary specification applies to goods the consumption composition that Moro et al. (2017) use to split the services sector into market services and home services.

From the first order conditions, we derive the following sectoral expenditure share equations ${ }^{53}$ :

$$
\begin{aligned}
s_{s}\left(\mathbf{p}_{\mathbf{t}}, \hat{e}_{t}\right) \equiv \frac{p_{t, s} \hat{c}_{t, s}}{\hat{e}_{t}}=\frac{\left(1-\varphi_{g}\right) p_{s, t}^{1-\varepsilon} \Phi_{t, 1}}{\Phi_{t, 2}} \\
s_{g_{d}}\left(\mathbf{p}_{\mathbf{t}}, \hat{e}_{t}\right) \equiv \frac{p_{t, g_{d}} \hat{c}_{t, g_{d}}}{\hat{e}_{t}}=\frac{\varphi_{g} p_{g_{d}, t}^{1-\varepsilon} \phi_{g_{d}}^{\varepsilon} \Omega_{t, 1}^{\frac{\varepsilon}{\gamma}-1} \Phi_{t, 1}}{\Phi_{t, 2}}-\frac{p_{g_{d}, t} \Omega_{t, 1}^{-1} \hat{\bar{c}}_{g}}{\hat{e}_{t}} \\
s_{g_{n d}}\left(\mathbf{p}_{\mathbf{t}}, \hat{e}_{t}\right) \equiv \frac{p_{t, g_{n d}} \hat{c}_{t, g_{n d}}}{\hat{e}_{t}}=1-s_{s}\left(\mathbf{p}_{\mathbf{t}}, \hat{e}_{t}\right)-s_{g_{d}}\left(\mathbf{p}_{\mathbf{t}}, \hat{e}_{t}\right)
\end{aligned}
$$

where

$$
\begin{aligned}
\Phi_{t, 1} & \equiv\left(1+\frac{p_{t, g_{n d}} \hat{\bar{c}}_{g_{n d}}+p_{g_{d}, t} \Omega_{t, 1}^{-1} \hat{\bar{c}}_{g}+p_{g_{n d}, t} \Omega_{t, 2}^{-1} \hat{\bar{c}}_{g}}{\hat{e}_{t}}\right), \\
\Phi_{t, 2} & \equiv\left(1-\varphi_{g}\right) p_{s, t}^{1-\varepsilon}+\varphi_{g} p_{g_{d}, t}^{1-\varepsilon} \phi_{g_{d}}^{\varepsilon} \Omega_{t, 1}^{\frac{\varepsilon}{\gamma}-1}+\varphi_{g} p_{g_{n d}, t}^{1-\varepsilon}\left(1-\phi_{g_{d}}\right)^{\varepsilon} \Omega_{t, 2}^{\frac{\varepsilon}{\gamma}-1},
\end{aligned}
$$

${ }^{52}$ Remind that we follow Matsuyama (2016) by imposing $\hat{\bar{c}}_{s}=0$.

${ }^{53}$ The shares arise from the following compensated demands: $\hat{c}_{s, t}=\left(1-\varphi_{g}\right)\left(\frac{p_{t}}{p_{t, s}}\right)^{\varepsilon} \hat{c}_{t}$; $\hat{c}_{t, g_{d}}=\varphi_{g}^{\frac{\gamma}{\epsilon}} \phi_{g_{d}}^{\gamma} \hat{c}_{g, t}^{\frac{\varepsilon-\gamma}{\varepsilon}}\left(\frac{p_{t}}{p_{t, g_{d}}}\right)^{\varepsilon} \hat{c}_{t}^{\frac{\gamma}{\epsilon}}$ and $\hat{c}_{t, g_{d}}=\varphi_{g}^{\frac{\gamma}{\epsilon}}\left(1-\phi_{g_{d}}\right)^{\gamma} \hat{c}_{g, t}^{\frac{\varepsilon-\gamma}{\varepsilon}}\left(\frac{p_{t}}{p_{t, g_{n d}}}\right)^{\varepsilon} \hat{c}_{t}^{\frac{\gamma}{\epsilon}}$, where $\hat{c}_{g, t}=$ $\varphi_{g}\left(\frac{p_{t}}{p_{t, g}}\right)^{\varepsilon} \hat{c}_{t}$ 
and where

$$
\begin{aligned}
\Omega_{t, 1} & \equiv\left[\phi_{g_{d}}+\left(1-\phi_{g_{d}}\right)\left(\frac{1-\phi_{g_{d}}}{\phi_{g_{d}}}\right)^{\gamma-1}\left(\frac{p_{t, g_{d}}}{p_{t, g_{n d}}}\right)^{\gamma-1}\right]^{\frac{\gamma}{1-\gamma}}, \\
\Omega_{t, 1} & \equiv\left[\left(1-\phi_{g_{d}}\right)+\phi_{g_{d}}\left(\frac{\phi_{g_{d}}}{1-\phi_{g_{d}}}\right)^{\gamma-1}\left(\frac{p_{t, g_{n d}}}{p_{t, g_{d}}}\right)^{\gamma-1}\right]^{\frac{\gamma}{1-\gamma}} .
\end{aligned}
$$

With respect to technology, equations (6) - (13), and market clearing conditions $(14)-(16)$, there is one slight modification related to the number of sectors due to the split of the broad goods sector into nondurable and durable goods sectors. That is, although $m^{\prime} \in M^{\prime} \equiv\{d\} \cup F$, where again $d$ stands for the investment sector and $F=\left\{g_{d}, g_{n d}, s\right\}$, specification of sectoral production function remains the same as in section 2. One additional modification refers to the relative price of goods in equation (12) on the price of the composite consumption, which again arises manipulating the first order conditions of the households intratemporal problem, so that:

$$
p_{t} \equiv\left[\varphi_{g} p_{t, g}^{1-\varepsilon}+\left(1-\varphi_{g}\right) p_{t, s}^{1-\varepsilon}\right]^{\frac{1}{1-\varepsilon}},
$$

where $p_{t, g} \equiv\left[\phi_{g_{d}}^{\gamma} p_{t, g_{d}}^{1-\gamma}+\left(1-\phi_{g_{d}}\right)^{\gamma} p_{t, g_{n d}}^{1-\gamma}\right]^{\frac{1}{1-\gamma}}$.

We follow a similar calibration strategy relative to the baseline model. We keep almost all parameters the same as the previous baseline case, which implies that the model features the same level of aggregate capital in the new stationary equilibrium as in the baseline case. Stone-Geary preferences parameters are different relative to the baseline case since they feed the model calibration stemming from another estimation including price and finalconsumption data from NIPA-BEA from 1947 to $2010^{54}$ following the same methodology we apply to the baseline case. We also apply similar consistency with BLS data. The estimation of the preference paramenters of the extended Stone-geary preferences shows that by including another nonhomotheticity term for nondurable goods, $\bar{c}_{g_{n d}}$, it turns out that the nonhomotheticity term of the broad goods sector, $\bar{c}_{g}$, becomes very small and statistically insignificant (see table A.1 in Appendix A). It then reinforces our interpretation that this term represents a nondurable subsistence - particularly food subsistence, even though the model does not feature a sector of agriculture. Thus, we follow the estimation by setting $\bar{c}_{g}=0$ in the model calibration.

Again, $\hat{\bar{c}}_{g_{n d}, 1947}$ and $\psi_{f}, f \in F=\left\{g_{d}, g_{n d}, s\right\}$ were calibrated ${ }^{55}$ so that the sequence of $\hat{\bar{c}}_{g_{n d}}$ yields the moments estimated in data similarly as shows

\footnotetext{
${ }^{54}$ See appendix D on the estimation implementation.

${ }^{55}$ We set $\psi_{s}=0.01 ; \psi_{g_{d}}=0.025 ; \psi_{g_{n d}}=0.037$.
} 
table 3 (see in table A.2 in appendix A for these targeted moments). Remind that we leave both initial level of sectoral labor-aumenting productivity and sectoral technological progress free to match aggregate sectoral expenditure shares in initial year, 1947. Note that in this extended baseline case, in computing aggregate expenditure shares of nondurable goods and services sectors we do not consider durable goods for consistency with data, in which we exclude durables to mitigate mis-measurement on CE-BLS data. Therefore, the ambition of this extension is that by targeting data on aggregate sectoral expenditure shares of nondurable consumption that is consistent with micro data, it implies that the fit of the Engel's law on nondurable consumption improves in the model.

Overall, our baseline model extension succeeded in improving the match to data since the framework can reasonably replicate the observed structural change quantitatively. That is, not only relative price of nondurable goods and the Kuznets facts (see figures 9 and 10), but specially the implied crosssectional variation in the expenditure structure grouped by income quintiles -Engel's law - are confirmed by the data, as shows figure 11. 


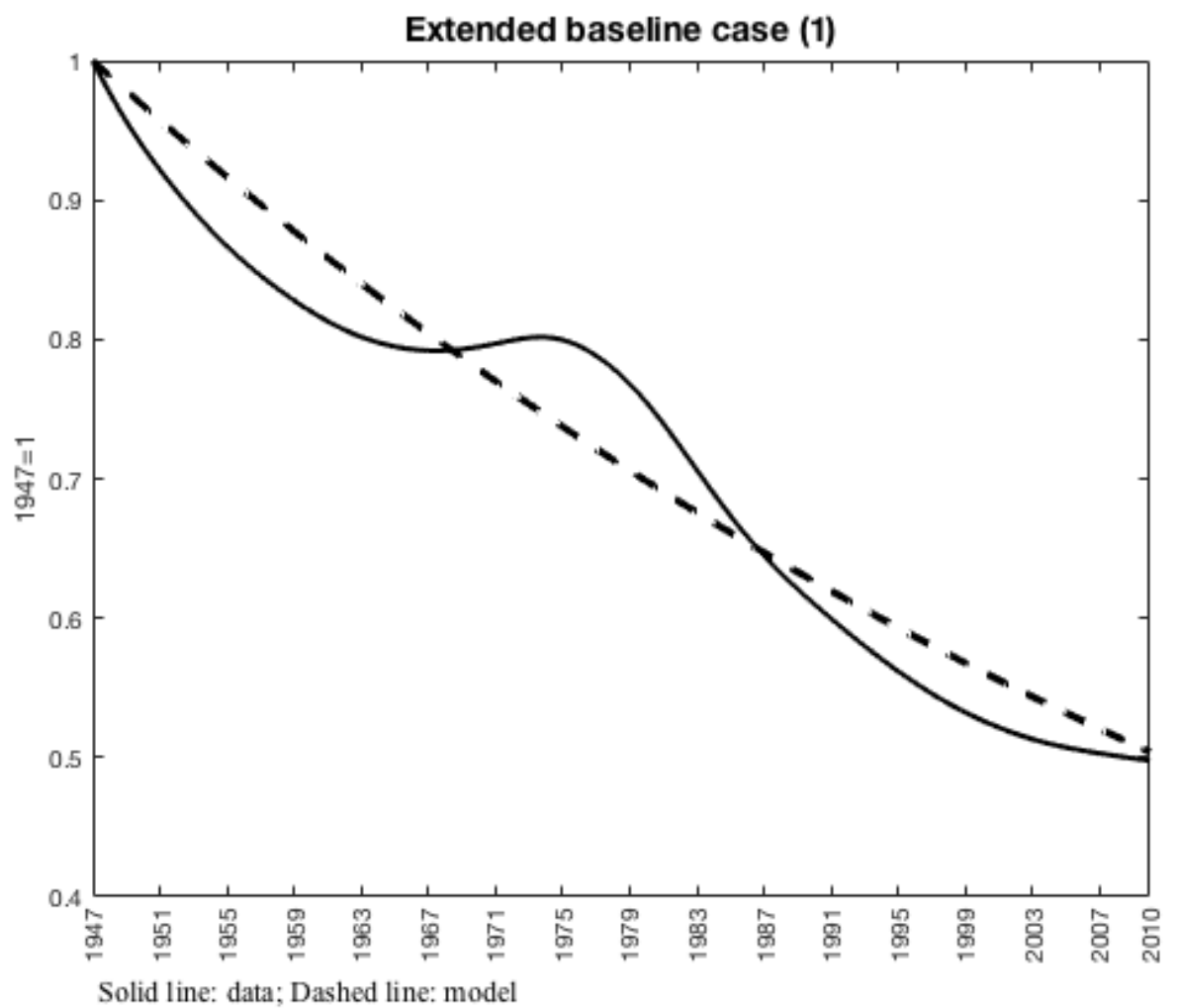

Figure 2.9: Fit of the Model to Goods to Services Prices Ratio in the Postwar US

Notes: This figure depicts the fit of the extended baseline model to the ratio of nondurable goods to services prices using data from NIPA-BEA for goods (agriculture and manufacturing) and services. Data follows Herrendorf et al. (2009) subject to smaller consistency issues due to data source differences with expenditure data from CE-BLS and price data from CPI-BLS. Definition of sectors and its components with data from CPI-BLS matches to sectoral definitions with data from CE-BLS. Solid and dashed lines denote, respectively, data from NIPA-BEA (smoothed with Hodrick-Prescott filter) and the model prediction. See the data appendix for further details on definitions of expenditure sectors. 


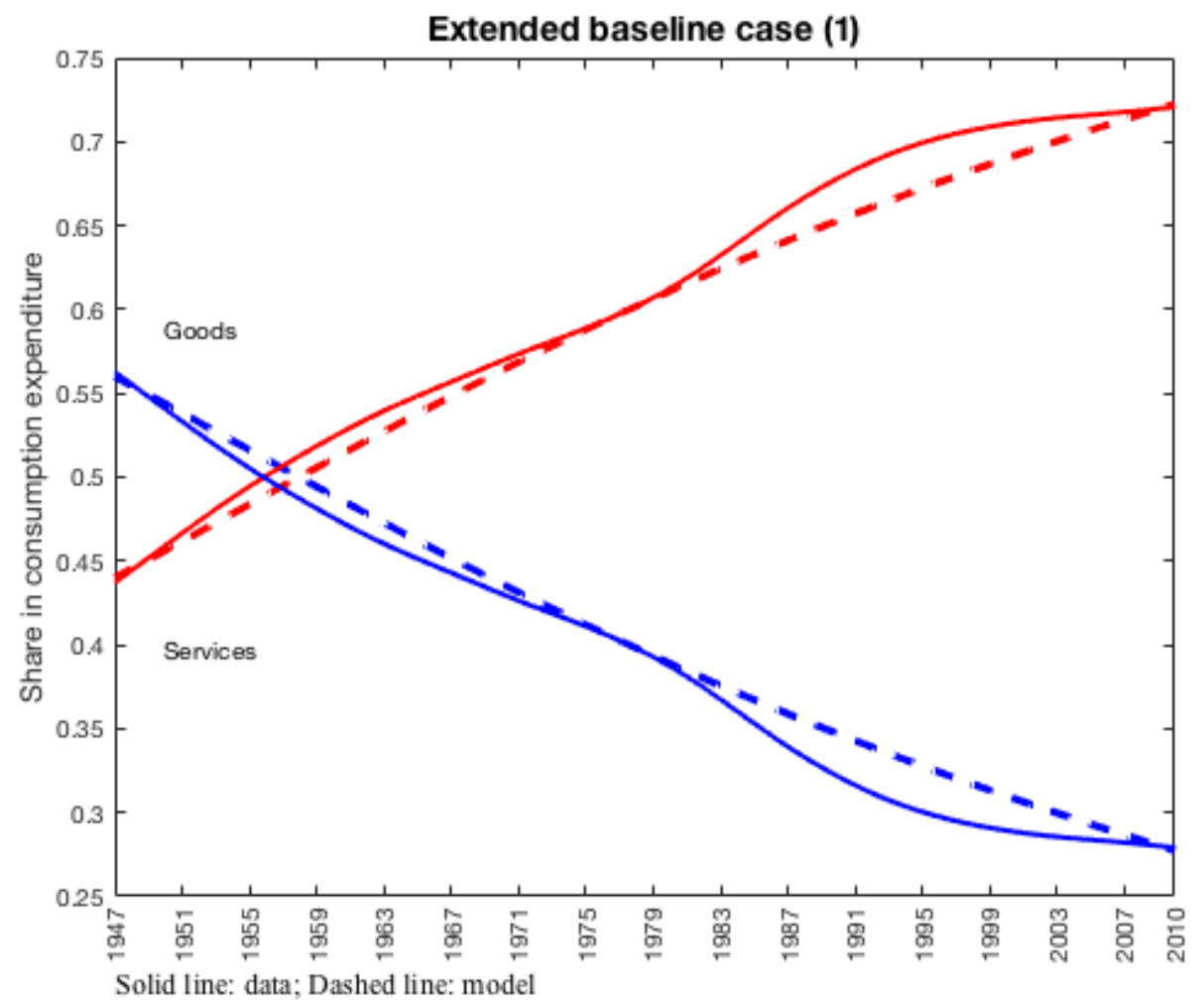

Figure 2.10: Fit of the Extended Model to Kuznets Facts in the Postwar US

Notes: This figure depicts the fit of the extended model to the aggregate sectoral shares of nondurable consumption expenditure - Kuznets facts - using data from NIPA-BEA in 1947-2010 (smoothed with Hodrick-Prescott filter) for goods (agriculture and manufacturing) and services following Herrendorf et al. (2009) subject to smaller consistency issues due to data source differences. Solid and dashed lines denote, respectively, data from NIPA-BEA and the model prediction. See the data appendix for further details on definitions of expenditure sectors.

Lastly, note that although Stone-Geary preferences is well-suited to represent developments of the Kuznets facts in the postwar US, it presents a caveat in depicting the Engel's law dynamics according to available data in the past three decades. It is known that Stone-Geary preferences limits the explanatory power of the income effects in the long-run since Engel curves level off asymptoticaly (Buera and Kaboski, 2009; Comin et al., 2015). Consequently, as we see in figure 11, the level difference of the average sectoral shares of income quintiles slowly declines over time since the relevance of the nonhomotheticity term, which gives rise to income effects, falls as the economy grows, so that the level difference of the average sectoral shares of income quintiles vanishes asymptotically.

Although the time range is limited by available data since the 80's and then it is challenging to raise a more assertive conclusion, a simple look at the data does not confirm this pattern arising from the Stone-Geary preferences. Instead, it suggests that the level difference of the average sectoral shares of income quintiles increases specially from mid-90's or at least remains stable, 
Chapter 2. Reconciling Kuznets Facts, Engel's Law and Relative Prices trend in the postwar

as suggests Boppart (2014). Note that Stone-Geary preferences seems to fit better the Engel's law in brazilian case, as shows figure A.6 in appendix A. Although there are only two periods available, one can see that, unlike US from the 80's, the level difference of the average sectoral shares of income quintiles declines over time. The difference between experiences in US between 1986-2010 and Brazil between 2003-2009 might be related to the fact that whereas the time range in the former was marked by an increase of income inequality, in the latter it is widely known as a period in which income inequality fell. However, a more thorough investigation of this pattern on Engel's law dynamics and its illustration with stable preferences with longrun income effects is left for future work. That is, for example, to investigate whether different Engel's law dynamics might reflect distinct experiences of income and consumption inequality and heterogeneity in income elasticities in the cross-section of households.
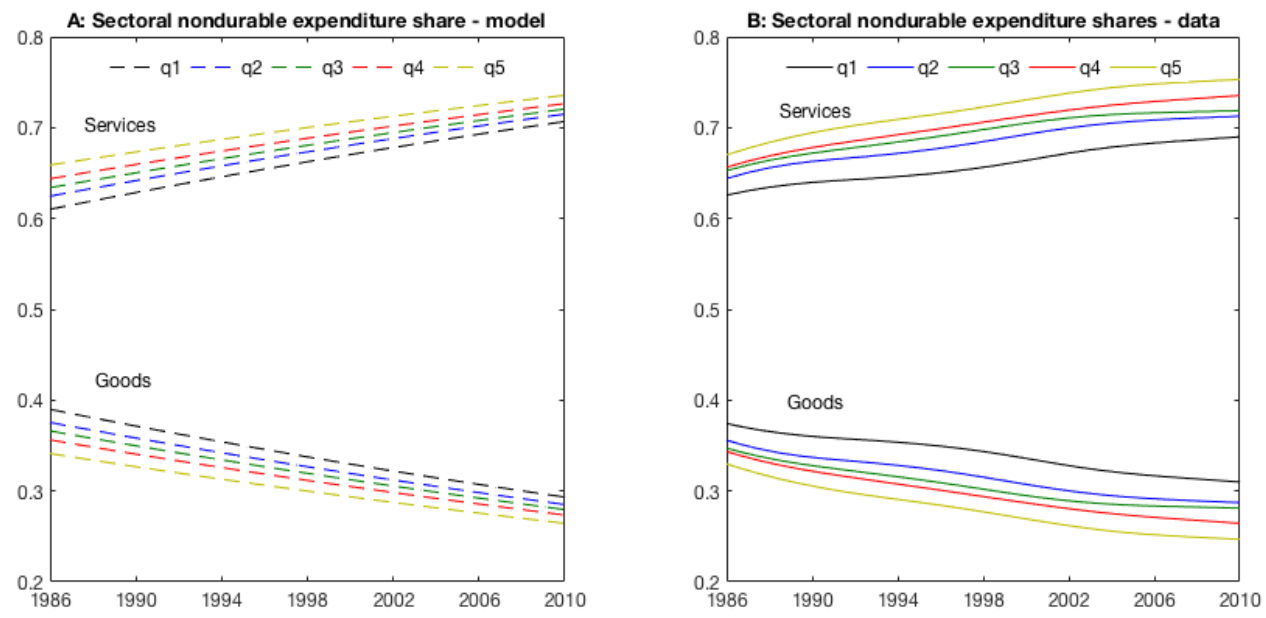

Figure 2.11: Engel's Law in the in the US Predicted by the Extended Baseline Model and According to Reported Nondurable Expenditure in CE-BLS -19862010

Notes: This figure depicts the sectoral nondurable consumption shares of households grouped by income quintiles -Engel's Law - predicted by the extended baseline model in panel A and presented by data from reported expenditures in the interviews of the CE-BLS for nondurable goods (agriculture and manufacturing) and services in panel B. Aggregate data on sectoral expenditure sahres from CE-BEA approximates in levels a measure of household sectoral expenditure shares with data from NIPA-BEA. See the data appendix for further details on definitions of expenditure sectors.

\section{4}

\section{Conclusion}

This paper presents a growth theory that is consistent with relative price dynamics, Kuznets facts and Engel's law in the postwar US. We do so by extending a canonical Bewley-Aiyagari model in continuous time in 
a tractable framework embedded with a two-sector environment without departing from benchmark Stone-Geary preferences. Bewley-Aiyagari models constitute a natural workhorse to study heterogeneity in household choices and market outcomes altogether. Therefore, representation of the Engel's law arises naturally by following demand patterns in the cross-section of households. In calibrating the model to represent the postwar US, our analysis offers two contributions.

First, our general equilibrium framework in a multi-sector environment with nonhomothetic preferences enables us to show that price and income effects drive structural change not only through intratemporal sectoral consumption demands. Relative prices and nonhomotheticity terms also drive structural change via intertemporal opportunities since they impact expenditure (and savings) policy functions. Second, the simulations with the three versions of the model suggest that the two driving forces of structural change in the aggregate shares of final consumption expenditure (Kuznets facts), price and income effects, are of roughly equal size in the postwar US. Moreover, we reinforce that reconciling these three main empirical regularities in the postwar US calls for a growth theory that accommodates both long-run demand (income effects) and supply (price effects) drivers of structural change.

Structural change and several other topics in economics are interconnected, such as, labor supply, biased technical change, consumption and income inequality. Works in these topics often follow either partial or general equilibrium approaches in standard one-sector models. To this respect, being tractable, easy to extend in many fronts and empirically plausible are appealing features of our framework to analyze these interrelations in a multi-sector environment.

The analysis we carry out can be extended in many dimensions. For example, it would be of interest to feed the model calibration by exploiting cross-sectional variation in the expenditure structure on microdata to estimate the model's key preference parameters instead of on aggregate data. Thus, allowing the model for heterogeneity in preferences, for example by households grouped by income quintiles, might improve the fit of the Engel's law to data by weakening the asymptotic homotheticity property of Stone-Geary preferences. Alternatively, one could apply preferences that feature long-run income effects so that income effects do not level off asymptotically since reconciling these three main stylized facts of structural change indeed calls for a growth theory that accommodates both long-run demand (income effects) and supply (price effects) drivers of structural change. Finally, it would be useful to extend the US postwar analysis we carry out here to other countries in different stages 
Chapter 2. Reconciling Kuznets Facts, Engel's Law and Relative Prices trend in the postwar US in a Bewley-Aiyagari Framework with Stone-Geary Preferences

of development not only to improve assessment of the relative relevance of driving forces of structural change but also for robustness check of the empirical regularities we highlight in this study. 


\section{Chapter 3}

\section{Follow the Money: The Effects of Liquidity Shocks on Con- sumption and Debt - Evidence from a Natural Experiment in Brazil}

\section{1 \\ Introduction}

This paper studies an unique policy experiment by the Brazilian government that is exogenous and allows one to discern an unanticipated announcement effect and an anticipated effect of the program's benefits in both consumption and debt. While there is substantial evidence that consumers respond to anticipated liquidity and income shocks by more than standard models would predict, evidence on unanticipated shocks are scarse since they are relatively rare events and even if a potentially unanticipated event arises, there are many underlying challenges in identifying shocks that are indeed exogeous and unanticipated. Furthermore, measuring the scope of the effects is also challenging. Even though there is a growing body of research based on high-frequency and integrated data, existing data typically capture only some dimensions of the effect with sufficient resolution. Hardly ever they measure spending and debt simultaneously with accuracy.

In the late 2016, Brazil was trying to resume growth following the worst recession in its documented history in 2015 and 2016, when real GDP per capita accumulated a 8.5 percent fall. In this special context, on December 22, 2016, in a surprise pronouncement of the former President of Brazil, the government announced the Withdrawals Program of inactive accounts of the Guarantee Fund for Time of Service (FGTS) as an attempt to boost the economy. FGTS is a compulsory fund financed by employers in the formal sector to their employees in Brazil. ${ }^{1}$ The amount in each account is proportional to the workers' life cycle wages and withdrawals are permitted in restricted situations, so that the inactive accounts of the FGTS can be characterized as an illiquid asset. However, it is an unconventional illiquid asset. The fund is

\footnotetext{
${ }^{1} \mathrm{~A}$ workers' account becomes inactive when he or she quits or is dismissed for just cause.
} 
Chapter 3. Follow the Money: The Effects of Liquidity Shocks on Consumption and Debt -

used by the government in subsidized credit lines to specific industries and it yields to the workers a lower return relative to the base rate.

The program allowed the workers to withdraw, for any purpose, the entire amount that they had in an inactive account created up to December 31, 2015. The amounts could be withdrawn following a timeline which defined 5 groups according to workers' months of birth. Each group of treated workers was allowed to perform withdrawals in five subsequent initial months from March to July 2017. Interestingly, this positive liquidity shock mingles with an income shock due to the income effect associated to the interest rates differential between the FGTS accounts and the base rate in the economy. The program totaled US $\$ 13.6$ billion, which represents 0.7 percent of the annual gross domestic product (GDP) of Brazil in 2017. The program benefited 25.99 million workers with average withdraw of US\$523, which is equivalent to 80 percent of the monthly average earnings of employed people in Brazil in this year.

We use a unique panel dataset that merges three different databases with individual-level administrative records. Using de-identified individual public registration number we merge a dataset containing all withdrawals and its respective amounts made by participants of the program to another database that contains a rich set of individual demographic and labor contract characteristics to a third database containing all individual credit information in the brazilian banking sector. We end up with a final dataset containing 12.15 million treated workers (approximately 47 percent of total workers who qualified for the program, which accounts for 64 percent of total withdrawals) in a two-year sample period from 2016:01-2017:12.

We take advantage of this database to study how workers responded to this positive liquidity-income shock. We define a proxy of consumption that adds up credit card spending and down payments of vehicles and real estate financing. The balance of the remaining debt modalities is considered simply total debt ${ }^{2}$. There are several debt modalities comprising total debt in our database, such as installment credit, rural credit, personal credit, etc. In this work we highlight in the data description and in the results on modalities heterogeneity only the balance of the most relevant modalities of individual

\footnotetext{
${ }^{2}$ More precisely, our concept of total debt comprises all debt modalities excluding credit card spending, vehicles debt and real estate debt.
} 
Chapter 3. Follow the Money: The Effects of Liquidity Shocks on Consumption and Debt -

debt: overdraft debt ${ }^{3}$, credit card debt ${ }^{4}$ and payroll debt ${ }^{5}$. The three modalities jointly represent nearly 60 percent of individual total debt on average in $2017^{6}$. We also include balance of total overdue debt and total default debt ${ }^{7}$ to emphasize the program responses in terms of individual deleveraging in the Brazilian recessive context. Our methodology is based on a difference-indifferences identification applied to each of the five groups of treated workers. It exploits the program's qualification criteria - workers with inactive accounts of the FGTS created after December 31, 2015 or workers who cleared out their inactive accounts before the program announcement did not qualify to the program and thus comprise the control cluster for each group or treated workers following the same months of births defining these groups ${ }^{8}$.

We estimate a distributed lag model using the announcement date of the withdrawals program of the FGTS as the exogenous event. We summarize our findings as follows. First, we find that treated workers' consumption rose after the program announcement. For each $\$ 1$ withdrawn, treated workers on average spent $\$ 0.53$ during the months after the announcement (averaged across the five groups of treated workers $)^{9}$. In particular, $\$ 0.11$ for each $\$ 1$ withdrawn were consumed within the announcement window (or equivalently 25 percent of the cumulative consumption response, majorly via credit card spending), which represents a strong announcement effect. The life-cycle theory has a clear prediction that consumers should respond to the announcement (of an unanticipated income shock). Thefore, this result allowed us to neatly test this theory similarly to Agarwal and Qian (2014).

Second, the withdrawals program of the FGTS also implied a deleveraging effect after the withdrawals releases. Treated workers on average reduced total debt by $\$ 0.07$ for each $\$ 1$ withdrawn. In the months prior to the initial month of withdrawals releases, from which debt response start to decline, we find evidence of leveraging on overdraft and credit card debt modalities. That

${ }^{3}$ Overdraft debt is the amount readly available by a checking account. It accounted for notables 329 percent annual interest rate in December 2016.

${ }^{4}$ When a consumer does not fully pay the credit card bill at the due date or pays only part of it, the full or remainder due amount becomes automatically part of this modality. It accounted for notable 498 percent annual interest rate in December 2016.

${ }^{5}$ In the payroll debt, the debt payment is automatically charged from the individual salary. It is relatively 'cheap', accounting for a 29 percent annual interest rate, and represents more than 40 percent of individual total debt.

${ }^{6}$ Credit card debt accounts or around 5 percent and overdraft debt accounts for 10 percent of the individual total debt in 2017.

${ }^{7}$ Total overdue debt stands for overdue debt in any modality. The same applies to total default debt.

${ }^{8}$ As an attempt to control for seasonal consumption/savings behaviour related to birth months.

${ }^{9}$ We shall see in section 3 that the months after the announcement for groups 1-5 of treated workers are 9 to 13 months (2016:12 to 2017:08,.., 2016:12 to 2017:12, respectively). 
Chapter 3. Follow the Money: The Effects of Liquidity Shocks on Consumption and Debt -

is, indebted consumers used short-term liquidity in debt modalities in addition to credit card spending to smooth consumption, which is mostly overlooked in the literature on methods of smoothing consumption.

Third, consumption and debt responses were heterogeneous across spending categories and debt modalities. We find that consumption rose primarily in credit card spending after the program announcement (on average 70 percent of the consumption response) followed by the increase of big-ticket durables, specially real estate down payments (on average 26 percent of the consumption response), which is consistent with prior research (e.g.Aaronson et al. (2012),Parker et al. (2011)), considering the relative size of the shock, and is suggestive of an important role for liquidity constraints, as the unanticipated shock may have provided otherwise unavailable down payments for debt-financed purchases of durables. With respect to debt, payroll debt stood out among modalities, accounting for 48 percent of total debt response after the program announcement. Lastly, consumption response was heterogeneous across individuals. Constrained consumers, measured as young or old, showed stronger consumption responses.

Our paper builds on three literatures. The first is the literature that introduces an additional illiquid asset to benchmark (liquid and risk-free) oneasset economies (e.g Kaplan and Violante (2011),Kaplan et al. (2014),Kaplan et al. (2016)) to study the behaviour of wealthy hand-to-mouth individuals and economic policy by considering these non-Ricardian individuals. It provides a framework to think of the liquidity shock to the FGTS asset. Note, however, that an inactive account of the FGTS represents an unusual illiquid asset for this literature. In this two-asset environment, households may choose to hold an illiquid asset that typically yields a higher return, but it can only be accessed by paying a transaction cost. In the FGTS case, it is compulsory (exogenous) and yields a lower return relative to the base rate. Based on insights from this framework, an immediate, direct effect of this liquidity-income shock is an increase of the disposable income arising from the interest rate differential between the one that remunerates the FGTS' resources and the base rate on the liquid and risk-free asset. Constrained consumers rise consumption and reduce debt reflecting the relaxed borrowing (or liquidity) constraints, while unconstrained consumers may either increase consumption or rebalance their portfolio depending on income and substitution effects following general equilibrium multipliers and price effects.

Second, our work is related to the literature that address the effects of a shock to liquidity. For example, Baker and Yannelis (2017) and Gelman et al. (2018) analyzes the response to the liquidity shock caused by the 2013 US 
Chapter 3. Follow the Money: The Effects of Liquidity Shocks on Consumption and Debt -

government shutdown using data derived from account records linked to an online banking app. Baker and Yannelis (2017) emphasizes the heterogeneous spending response by category of spending, while Gelman et al. (2018)shows that many individuals who had low liquid assets used multiple sources of short-term liquidity to smooth consumption. Both studies identify a pure liquidity shock holding income constant. In our case shocks to income and liquidity arrive together. Rather than distinguishing the response to a change in liquidity from the response to a change in resources, our focus is to measure the overall response of the withdrawals program of the FGTS on consumption and debt and to test the life-cycle theory. However, interpreting these responses remains challenging for the recent studies on excess sensitivity of consumption to income.

Having said this and considering the above mentioned peculiarity of the FGTS iliquid asset, this paper is more closed related to studies of consumption and debt response to either anticipated and temporary changes in income and unanticipated and temporary changes in income. The former group of the literature has explained the response to consumption of these expected income shocks through models of liquidity constraints and precautionary savings. It includes: Carroll (1992),Parker (1999),Shapiro and Slemrod (2001),Souleles (2002),Hsieh (2003), Stephens (2003),Johnson et al. (2004), Agarwal et al. (2007),Stephens and Unayama (2010),Parker et al. (2011) Carroll (1997); For example, Johnson, Parker, and Souleles (2006) and Parker et al. (2011) study the effects of the tax rabates in 2001 and 2008 in the US on consumption using the CE-BLS sample. Agarwal, Liu, and Souleles (2007) uses a high-frequnecy micro-data sample of credit card spending to assess the consumption effect of the tax rebate in 2001 in the US. These studies on US stimulus policies take advantage of clean identification from the random payout timing.

A few papers that study the consumption response to unanticipated and temporary changes in income are Woplin (1982); Paxson (1993) ; Gruber (1997); and Jappelli and Pistaferri (2014). For a review of the literature, see Browning and Crossley (2001)and Jappelli and Pistaferri (2010). Particularly, Jappelli and Pistaferri (2010) documents that anticipated and unanticipated income shocks bear different implications for the consumption response. Permanent income hypothesis (PIH) suggests that consumption should respond to an unexpected increase in income and the magnitude of the consumption response is equal to the real interest rate in a complete market with an infinite horizon but will be higher when the horizon is finite. Furthermore, the consumption response could be significant when consumers face borrowing or liquidity constraints or when precautionary saving motives are strong (Zeldes 
Chapter 3. Follow the Money: The Effects of Liquidity Shocks on Consumption and Debt -

(1989),Carroll (1992)) . Prior studies (e.g., Poterba (1988)) have had difficulties in identifying the announcement effect mainly due to low frequency or lack of cross-sectional variation, or the fact that the policy announcement was not really a surprise. Other studies that use temporary job loss as identification are potentially subject to endogeneity and external validity concerns (Gruber, 1997). The setting in Agarwal and Qian (2014), which is the closest study to ours, was the first to cleanly test life-cycle theory by decomposing the postpolicy window into the announcement period and the disbursement period in a policy experiment in Singapore.

Finally, we acknowledge that the advisability of the use of this program for public policy depends crucially on the extent to which these withdrawal resources directly changed individual spending, as well as on any subsequent multiplier or price effects. While we estimate substantial direct, partial equilibrium effects, admittedly, the ultimate impact of the program on aggregate consumption may be higher or lower than implied by these calculations, due to possible indirect, general equilibrium changes in prices or interest rates and in additional spending through multiplier effects.

The rest of the paper follows. Section 2 describes the withdrawals program of the FGTS. Section 3 discusses the data and empirical strategy on the econometric methodology. Results are reported in section 4. Section 5 concludes.

\section{2}

\section{Withdrawals Program of the FGTS}

In the special recessive context of the Brazilian economy we have mentioned in the introduction, the former President of Brazil announced in pronouncement on December 22, 2016 that, in an attempt to boost the economy, the government was releasing withdrawals from inactive accounts ${ }^{10}$ of a government-controlled compulsory worker fund - the Guarantee Fund for Time of Service (FGTS) ${ }^{11}$ - for terminated employment contracts until 31 December $2015^{12}$. Workers cannot freely access the funds from these inactive

\footnotetext{
${ }^{10}$ The account becomes inactive when a employee quits or is dismissed for just cause.

${ }^{11}$ FGTS is a compulsory fund created in 1966 imposed by the government to formal employment contracts so that employers deposit in open accounts at "Caixa EconÃtmica Federal" (a government-owned financial institution), at the beginning of each month and in the name of employees, the amount corresponding to 8 percent of the wage of each employee.

${ }^{12}$ As stipulated by provisional executive order $n$. 763/2016.
} 
Chapter 3. Follow the Money: The Effects of Liquidity Shocks on Consumption and Debt -

accounts. Withdrawals are permitted in restricted situations ${ }^{13}$, which means an inactive accout of the FGTS is an illiquid asset. To this respect, the withdrawal release program in 2017 represents a liquidity shock to beneficiaries. Nevertheless, it is an unusual illiquid asset since it is compulsory and remunerated with low interest rates - lower than the base rate set by the Central Bank of Brazil - SELIC ${ }^{14}$. Therefore, we argue that in this particular case, the liquidity shock is equivalent to an income shock due to the income effect associated to the interest rates differential between the FGTS accounts and any other asset in the economy. In fact, these accounts have been accumulating real losses at least for the past two decades. In appendix B we develop a simple two-period consumption model that formalizes this intuition on the FGTS liquidity-income shock and the MPC responses of hand-to-mouth (HtM) and non hand-to-mouth (N-HtM) consumers.

While the amount each beneficiary was allowed to withdraw was proportional to his or her life cycle wages, the average withdrawal was US $\$ 523^{15}$. It worths mentioning that the size of the shock was substantial considering a middle income economy. The average withdrawal accounts for 80 percent of monthly average earnings of employed people in Brazil in 2017. As a matter of comparison, the growth dividend that a typical qualified Singaporean received in the growth divident program in Singapure in Agarwal and Qian (2014) accounted for 18 percent of monthly median income in Singapore in 2011. Approximately 25.99 million people benefited from the withdrawal program of the FGTS in Brazil - around 30 percent of the labor force -, which implies the program totaled US $\$ 13.6$ billion. This amount accounted for aproximatelly 1.2 percent of the aggregate household consumption expenditure ${ }^{16}$ and 0.7 percent of the brazilian GDP in 2017, which is comparable to the size of the 2001 and 2008 US tax rebates (Johnson et al, 2006; Johnson et al, 2013).

Withdrawals had no limit, which means the the worker could withdraw,

${ }^{13}$ The situations can be summarized as follow: i. dismissal without just cause; ii. termination of contract; iii. retirement; iv. death; v. permanence outside the FGTS regime for three years in a row; vi. purchase of a property, liquidation, amortization or mortgage payment; vii. serious illness; viii. company extinction, ix. termination of contract by reciprocal fault, $\mathrm{x}$. personal need in case of emergency or serious reasons linked to natural disaster and xi. suspension of odd job.

${ }^{14}$ The FGTS accounts yield 3 percent per year plus the reference rate (TR). The TR is used as a reference for interest rates in Brazil and makes the monetary correction of various types of investment, such as savings. In fact, these accounts have been accumulating real losses in the past two decades. The fund is used by the government in subsidized credit lines in the housing sector.

${ }^{15}$ The exchange rate applied is US $\$ 1=\mathrm{BRL} \$ 3.2591$ as of December 2016 -Exchange rate - Free - United States dollar (sale) - end of period from the Central Bank of Brazil. All other amounts in (BRL\$) applies this exchange rate henceforth.

${ }^{16}$ According to the National Accounts System of the Brazilian Institute of Geography and Statistics (IBGE) - excluding imputed rent for dwelling services. 
Chapter 3. Follow the Money: The Effects of Liquidity Shocks on Consumption and Debt -

for any purpose, the entire amount that he or she had in the inactive account. The criterium for determining the timeline for the withdrawals was exogenously defined - took into account the date of birth of the workers who qualified for the program. Five groups were created according to the month of birth: (1) born in January and February were able to withdraw as of March 10, 2017; (2) born in March, April and May, from April 10, 2017; (3) June, July and August, beginning on May 12, 2017; (4) born in September, October and November, from June 16, 2017; and (5) born in December, from July 14, 2017. The deadline for any withdrawal was July 31.

Similarly to the growth dividend program in Singapore and unlike other stimulus programs such as tax rebates in the United States, we found evidence supporting that the withdrawals program of inactive accounts from the FGTS in Brazil was unanticipated. We perform a through search on the web media related to the program and find no information one-year period before the announcement. We also perform a Google Trends search in a 30-month period comprising the announcement and timeline of the program. We see in Figure 3.1 figure 1 that before the announcement date virtually no search were done. On the other hand, searches for the program become more salient on the web from this date through the withdrawals deadline. All major newspapers media also start to cover the stimulus program, publicizing that the worker could: (i) consult the account balance on the website of the Caixa EconÂtmica Federal (a government-owned financial institution that manages the fund), (ii) on the website of the FGTS itself, (iii) or through an app for smartphones and tablets and (iv) it was also possible to make a registration to receive information from the FGTS by messages in the mobile or by e-mail.

Therefore, as theory has different predictions for the two effects, our policy experiment also enable us to assess effects of announcement (unanticipated) and withdrawals (anticipated) periods for the five groups of the program benefeciaries, instead in a middle-income continental country in a recessive context.

\section{3}

\section{Data and Methodology}

\subsection{1}

Data

In our analysis we merge three individual-level datasets with administrative records using the public individual registration number ${ }^{17}$ to link individuals in these datasets. The first dataset is obtained from the government-owned

\footnotetext{
${ }^{17}$ Datasets are subject to individuals confidenciality and were obtained for research purposes with a de-identified number associated to the public individual registration number.
} 
Chapter 3. Follow the Money: The Effects of Liquidity Shocks on Consumption and Debt -

bank Caixa EconÃtmica Federal, which is the depository institution of the FGTS resources. This sample fully covers the 25.99 million workers who qualified for the program and performed withdrawals from their innactive accounts. It contains the birth date and the amount, date and number of withdrawals by each individual's inactive account during the withdrawals timeline, from March to July 2017.

Our dataset on consumer credit transactions is obtained in the Credit Information System (SCR) from the Central Bank of Brazil (CBB) in a 24-month period range between 2016:01 and 2017:12. The SCR is a registration dataset managed by the CBB that is fed monthly by all financial institutions in Brazil, which register all credit operations in the country ${ }^{18}$. The database contains transaction-level information on all credit/debt modalities and some demographic characteristcs, including age, property address, zip code and county. For the purpose of this analysis, we aggregate the data at the individual-month level. The variety of credit/debt modalities includes total balance, balance of new transactions, interest rates, spreads, overdue balance, etc. In this study we split the modalities into two groups. First we add credit card spending ${ }^{19}$ to two other consumption proxies related to durables spending. We define down payments of vehicles and real estate financing as the difference between the balance of debt in these two modalities and the value of a non surety guarantee as an attempt to approximate the balance of vehicle and real estate debt modalities to an amount that is indeed consumed. The balance of the remaining modalities are considered simply debt. Therefore, the proxy of consumption that we have available adds up credit card spending and down payments of vehicles and real estate financing. Furthermore, we highlight in the data description and in the results the relatively more relevant modalities of individual debt which comprises: balance of overdraft debt ${ }^{20}$, balance of credit card $\operatorname{debt}^{21}$ and balance of payroll debt ${ }^{22}$. The first two modalities are the most expansive ones, accounting for notables 329 and 498 percent annual interest rates in December 2016. The latter although relatively 'cheap', accounting for a 29 percent annual interest rate, represents more than 40 percent of individual total debt. The three modalities jointly represents nearly 60 percent of individual total

\footnotetext{
${ }^{18}$ More precisely, all credit operations above US\$61.4 (BRL $\left.\$ 200\right)$.

${ }^{19} \mathrm{It}$ is included as a credit modality in the SCR database. Close to 20 percent of aggregate household consumption expenditure in the country is purchased using credit card according to the National Accounts System - excluding imputed rent for dwelling services.

${ }^{20}$ Overdraft debt is the amount readly available by a checking account.

${ }^{21}$ When a consumer does not fully pay the credit card bill at the due date or pays only part of it, the full or remainder due amount becomes automatically part of this modality.

${ }^{22}$ In the payroll debt, the debt payment is automatically charged from the individual salary.
} 
Chapter 3. Follow the Money: The Effects of Liquidity Shocks on Consumption and Debt -

debt on average in $2017^{23}$. We also include balance of total overdue debt and total default debt $^{24}$ to emphasize the program responses in terms of individual deleveraging in a recessive context. Moreover, this modalities heterogeneity and demographic information allow us to assess differences in responses to the program by modalities and across (constrained and unconstrained) workers.

The other merged dataset is on labor market data, more precisely the Annual Report of Social Information (RAIS) from the Ministry of Economy. The RAIS is a dataset in which all employers must feed with many features of the job title and demographic information of the employees in formal labor contracts ${ }^{25}$. This database provides statistical information for government policy, including for the FGTS. It contains a rich set of demographics information about each individual, including earnings, age, date of birth, gender, race, schooling, occupation and information on the labor contracts, such as for example, when a employee starts to work, when either a employee quits or is dismissed and the reason why he or she is dismissed.

The datasets merge resulted in a intermediary dataset from which we exclude treated workers with no record on our concept of consumption or total debt in the SCR database during our sample period (i.e., 2016:01-2017:12). Moreover, to address the concern that outliers could obliterate overall responses and responses across groups, we also exclude workers in the extreme percentiles of both consumption and total debt. We were left with a final full dataset containing 11.9 million treated workers (46 percent of total workers who qualified for the program, which accounts for 64 percent of total withdrawals). These people account for a BRL $\$ 2,080$ (US\$638) average withdrawal, whereas the remaining people outside SCR account for around half of our sample's average withdrawal (BRL $\$ 1,154$ - US\$354). We use the first four months in our data (2016:01-2016:04) to identify consumers' pretreatment demographics and worker debt modalities. We then remove this range period

\footnotetext{
${ }^{23}$ Credit card debt accounts or around 5 percent and overdraft debt accounts for 10 percent of the individual total debt in 2017.

${ }^{24}$ Total overdue debt stands for overdue debt in any modality. The same applies to total default debt.

${ }^{25}$ All formal contracts in the private and public sectors. In Brazil a formal contract is in accordance with current labor legislation and, therefore, assuring the worker all the rights that he or she is entitled to. It basically comprises four types of job titles: i. job titles that have a labor card in the private of public sectors, which includes contributions to the general system of social security, withholding income tax - when there is a basis of incidence - on wage, deposits to the FGTS, overtime - when provided - with the legal increases, vacation release, etc. ii. job titles under the old public-own social security system or under the new public-own social security system; iii. military workers and iv. registered self-employed worker who pays due taxes and contributes to the general system of social security. In Brazil, as in many poor and emerging economies, there is a large share of informal jobs (around $40 \%$ ) in which employers do not pay due taxes and contributions and then employees do not take benefit of these rights provided by law.
} 
Chapter 3. Follow the Money: The Effects of Liquidity Shocks on Consumption and Debt -

from our sample for a cleaner identification. As a result, the final range period in our study is from 2016:5-2017:12.

By delimiting the scope of our analysis, it worths mentioning that we focus on consumption and debt effects of the program in the brazilian credit information system. Due to data limitations, we cannot track consumption and debt taken outside the banking system. We also do not track portfolio investment, which may be another destination of part of the withdrawals amounts, specially among richer workers. Furthermore, this study is based on individual information, hence it does not consider likely unfolded household effects.

The richness of the consumption and debt transaction-level information as well as the individual demographics in our dataset is that it allows us to better understand heterogeneity in consumption and debt response to the positive liquidity-income shock. For example, whether individuals spend and borrow differently and when they do so following a positive shock. To this respect, this data overcome substantial obstacles in terms of accuracy, scope and frequency that usually limits to obtaining reliable answers to these important issues. Although we do not have a detailed data on either credit card relative to studies that use micro-level credit card data (e.g., Gross and Souleles, 2002; Agarwal, Liu, and Souleles, 2007; Aaronson, Agarwal, and French, 2012) or jointly on credit card, debit card, and checking accounts (Agarwal and Qian (2014),Gelman et al. (2014),Baker (2018)), we have a more complete information on each individual indebtness in our sample. Rather than observing only aggregate debt or one specific debt modality, we have information on several modalities available in all banking system in the country. Moreover, relative to traditional household spending and balance-sheet datasets in the US such as the Survey of Consumer Finance (SCF), our sample is larger, allows high frequency analysis and have negligible measurement error.

Similarly to US stimulus policies, in our policy experiment qualified workers do not access the stimulus money at the same time. Meanwhile, unllike those programs, we cannot cleanly identify the stimulus effect from the random payout timing as as the specialized literature often carries out. In our case, it is the initial day of the withdrawal release that is exogenously defined according to the month of birth. Qualified workers do not receive a pre-defined amount of stimulus money by eletronic deposit in their checking account or by paper check, as in the US stimulus policies. They had to go to a bank agency to voluntarily withdraw it. We see in data that around 90 percent of the withdrawals occurs in the first month of release. However, the 
Chapter 3. Follow the Money: The Effects of Liquidity Shocks on Consumption and Debt -

exact total withdrawal amount and the day(s) it occurs possibly endogenously reflect qualified workers' demographic characteristcs or seasonal as well as the average of all other concurrent aggregate factors.

Therefore, instead of exploring variation on withdrawal timing, we use a difference-in-differences approach and rely on the untreated workers to identify the debt response. The untreated workers were raised as follows. In the RAIS database we have information on when a employee quits or is dismissed for just cause. Since these events define the deactivation of an account of the FGTS, it implies that we know whether he or she had an inactive account. We then tracked all 44.92 million individuals with formal labor contracts between 1994 to 2016 that had any inactive account in this period. Among these individuals, the ones who performed withdrawals, as indicated by the merge with the dataset of the Caixa EconÃtmica Federal with qualified workers' withdrawals, were assigned to the treatment cluster, while those individuals who did not qualified to the program due its participation criteria ${ }^{26}$ were considered the control cluster - nearly 18.92 million individuals. Moreover, we assign individuals in the control cluster to each of the five groups of treated workers following the months of birth as stated by the program timeline.

Tables 1 and 2 Tables 3.1 and 3.2 exhibit, respectively, summary statistics of demographics and consumption and debt variables, for the treatment and control clusters in the five groups of program benefeciaries. We see that the control cluster is not directly comparable with the treatment cluster along key dimensions in the full (unmatched) sample. For example, on average the control cluster is richer and more qualified than the treatment cluster. The amount available in the withdrawals program of the FGTS depends on individual's life cycle income, for which we use monthly income (labor earnings) in 2016 as proxy. Moreover, the level of education is also proxy for financial literacy. This suggests that the treatment cluster may have spending and debt patterns intrinsically different from that of the control cluster.

For a sound indentification of the policy effect, workers in the treatment and control clusters should also have comparable levels of income and education. Motivated by this evidence, we follow two approaches to address the challenge in statistical inference related to an unbalanced sample to report the results. First, we build a matched sample of workers in the treatment and control clusters that are similar in observable in demographic characteristcs and in consumption and indebtedness patterns. More precisely, we compute propensity scores based on a logistic regression using a set of income as well as

${ }^{26}$ That is, individuals with accounts created after December 31, 2015 or with accounts cleared by withdrawals made before the program announcement. 
Chapter 3. Follow the Money: The Effects of Liquidity Shocks on Consumption and Debt -

demographics, including age, schooling, gender and race. We also include average balances and interest rates of the main credit modalities at the individualmonth level as an attempt to capture unobservable characteristics as well as dummies for each of the 27 states as fixed effects to control for regional differences in a continental country as Brazil - see Table A.1 in appendix A for the logistic regression results. We then perform the nearest-neighbor matching based on the computed propensity scores. We compute the propensity scores in the first four months of our (pre-treatment) sample for each group of treated workers and then we remove this range period for a cleaner identification, as we mentioned. We see that differences in several characteristics either shrink significantly or become statistically insignificant in Tables 1 and 2 Tables 3.1 and 3.2. In addition to the mean statistics, distributions of age, monthly income in 2016 and credit card spending of the treatment and control groups after matching are also similar and comparable (Figures 2-4 Figures 3.2-3.4). Notwithstanding, we acknowledge that the matched sample might not eliminate the unobservable differences between treated and controlled workers, which would affect their consumption and debt behaviour.

In the second approach of our analysis, we consider all treated workers with some consumption and debt information in the SCR database. We exploit the estimated propensity scores by including them as regression weights in the full sample difference-in-differences analysis. The grounds behind it is to give a larger weight to those workers in the control cluster more similar to workers in the treatment cluster (e.g., those with similar income, age, educational level, etc.) in estimating the counterfactuals after the FGTS stimulus program.

The difference-in-differences identification approach requires the control group to have the same consumption and indebtedness patterns as the treatment group in the pretreatment period so that their behavior after the policy announcement constitutes a valid counterfactual. To illustrate it, we plot the unconditional means of treatment and control clusters of workers in the matched sample and in the full sample (weighted means by propensity scores) in all sample period (2016:01-2017:12) for the consumption proxy in Figure 5 Figure 3.5 and total debt in Figure 6 Figure 3.6. We see in these figures that the difference in consumption or total debt between the treatment cluster and the control cluster before the announcement of the withdrawal program of the FGTS remains virtually constant for all groups, which confirms the underlying identifying assumption of parallel pre-trends. Note that, while visual inspection is more subtle for consumption in the matched sample, in the full sample for both cosumption and total debt the lines depicting treatment and control visibly diverge after the program, which provides a suggestive evidence of the 
Chapter 3. Follow the Money: The Effects of Liquidity Shocks on Consumption and Debt -

workers response to the liquidity-income shock.

\subsection{2}

\section{Methodology}

We now assess the consumption and debt responses using a differencein-diferences regression approach that follows Agarwal and Qian (2014). Remind that the treatment cluster corresponds to workers who qualified for the FGTS program and performed withdrawals from their inactive accounts. The control group corresponds to workers that did not qualified for the program, that is, individuals whose accounts were created after December 31, 2015 or individuals who cleared up their inactive accounts by withdrawals made before the program announcement. The pretreatment period is from 2016:05 to 2016:11 (seven months), and the post-treatment period is from 2016:12 to 2017:12 (thirteen months).

The first specification shows the average monthly consumption or debt responses to the withdrawals program in each of the five groups of treated workers, according to the withdrawals timeline, where $g=\{1,2,3,4,5\}$ :

$$
Y_{i, t}^{g}=\beta_{\text {pre }}^{g} \times \$ W_{i}^{g} \times 1_{\text {pre }}+\beta_{\text {post }}^{g} \times \$ W_{i}^{g} \times 1_{\text {post }}+\alpha_{i}^{g}+\delta_{t}+\epsilon_{i, t} .
$$

The dependent variable, $Y_{i, t}^{g}$, is consumption or the balance amount of total debt for individual $i$ of group $g$, at the end of month $t$. $\$ W^{g}$ is the withdrawal amount of the FGTS Program that the worker $i$ in the treatment cluster of group $g$ did, and is equal to 0 for the control cluster. $1_{\text {pre }}$ is a dummy variable equal to 1 for the period before the announcement of the program (i.e, 2016:06-2016:11). ${ }^{27} 1_{\text {post }}$ is another dummy variable equal to 1 for the months after the announcement of the program (i.e, $\geq 2016: 12$ ). $\alpha_{i}^{g}$ is the individual dummy in each group $g$ used to absorb differences in individual preferences. $\delta_{t}$ is the year-month dymmy, included to absorb seasonal as well as the average of other concurrent aggregate factors. We clustered standard errors at the individual level.

$\beta_{p r e}^{g}$ captures the difference of consumption (or total debt) trend between the treatment and the control clusters of group $g$ in the pretreatment window, 2016:06-2016:11, (compared to the benchmark period, i.e, 2016:05). Note that $\beta_{\text {pre }}^{g}$ must be statiscally and economically indistinguishable from zero for validity of the difference-in-difference design. On the other hand, $\beta_{\text {post }}^{g}$ measures the average monthly post-policy reponse of consumption balance per

\footnotetext{
${ }^{27}$ Period 2016:05 is absorbed to identify the benchmark consumption or debt patterns in the estimation. We varied the number of absorbed months (2016:05-2016:06) and conversely the months before the announcement (2016:07-2016:11) and overall results are similar.
} 
Chapter 3. Follow the Money: The Effects of Liquidity Shocks on Consumption and Debt -

$\$ 1$ withdrawn by a treated worker (compared to the benchmark period) relative to the post-policy change of consumption of the control cluster in group $g$. $\beta_{\text {post }}^{g}$ has a different interpretation if $Y_{i, t}^{g}$ is total debt, since this dependent variable is a stock variable in levels, instead of a flow variable such as consumption. ${ }^{28}$ It then measures the average post-policy reponse (in the whole post-policy window) of total debt balance per $\$ 1$ withdrawn by a treated worker (compared to the benchmark period) relative to the post-policy change of total debt of the control cluster in group $g$.

In the following specification we allow for differences within the postpolicy period by splitting it into the announcement window and the withdrawal window:

$Y_{i, t}^{g}=\beta_{\text {pre }}^{g} \times \$ W_{i}^{g} \times 1_{\text {pre }}+\beta_{a}^{g} \times \$ W_{i}^{g} \times 1_{\text {announce }}^{g}+\beta_{w}^{g} \times \$ W_{i}^{g} \times 1_{\text {withdraw }}^{g}+\alpha_{i}^{g}+\delta_{t}+\epsilon_{i, t}$.

The dummy variables $1_{\text {announce }}^{g}$ and $1_{\text {withdraw }}^{g}$ equal to 1 for the months during the announcement and withdrawal windows, respectively. Note that both windows vary across groups of treated workers following the withdrawals timeline. More precisely, $1_{\text {announce }}^{g}$ equal to 1 for $g=\{1,2,3,4,5\}$ in the following month ranges: 2016:12-2017:02, 2016:12- 2017:03, 2016:12-2017:04, 2016:12-2017:05, 2016:12-2017:06, respectively. Since in the last group in the withdrawals timeline, $g=5$, we observe six months following the first month of this group's withdrawal release (2017:07) considering our sample period (2016:05 to 2017:12), we also set a 6-month withdrawal window for the remaining groups for comparability. Hence, $1_{\text {withdraw equal to }}^{g}$ 1 for $g=\{1,2,3,4,5\}$ in the following month ranges: 2016:03-2017:08, 2016:04-2017:09, 2016:05-2017:10, 2016:06-2017:11, 2016:07-2017:12, respectively. Therefore, $\beta_{a}$ and $\beta_{w}$ measure the average monthly post-policy (average in the whole post-policy window) response of consumption (total debt balance $)^{29}$ per $\$ 1$ withdrawn by a treated worker - compared to the benchmark period - in group $g$, relative to the change of consumption (or total debt) of group $g$ control cluster during the announcement and the withdrawal

${ }^{28}$ It applies to any stock variable in this study. We could alternatively define $Y_{i, t}^{g}$ as total debt change $\left(\triangle Y_{i, t}^{g} \equiv Y_{i, t}^{g}-Y_{i, t-1}^{g}\right)$. However, since in specification 1 we regress total debt on dummy variables of relatively large post-policy windows, $\beta_{\text {post }}^{g}$ in general become statistically insignificant since, we could see by applying specification 3 that, unlike the response of the flow variable of consumption, the debt change response is not persistent. In other words, the monthly coefficients are in general statistically significant only in the initial month of withdrawal release for each group of treated workers. Therefore, either the monthly sum of total debt change response or the average of the total debt response in levels in the post-policy windows are virtually identical responses for each group of treated workers. The choice for total debt in levels is for convenience due to specifications 1 and 2 .

${ }^{29}$ See footnote 21 for an explanation of the difference of interpretation of the response coefficients whether the dependent variable in the regression is consumption flow or the stock of total debt balance in levels. 
Chapter 3. Follow the Money: The Effects of Liquidity Shocks on Consumption and Debt -

windows, respectively.

The third specification is an event study approach on the dynamics of consumption (or total debt) responses. However, rather than identifying the effect through variation of the timing of the program disbursements, as usual in the literature, we do so in a difference-in-differences design. The following distributed lag model is estimated:

$$
Y_{i, t}^{g}=\sum_{s=-6}^{S^{g}} \beta_{s}^{g} \times \$ W_{i}^{g} \times 1_{\text {month } s}+\alpha_{i}^{g}+\delta_{t}+\epsilon_{i, t} .
$$

The coefficients $\beta_{-6}, \ldots, \beta_{-1}$ capture the difference of trends in debt modalities balance between the treatment cluster and the control cluster in the pretreatment months $(2016: 06, \ldots, 2016: 11)$, and we expect it to be economically and statistically insignificant. $\beta_{0}$ captures the immediate $\$$ response during the announcement month $(2016: 12)$. The marginal coeficients $\beta_{1}, \ldots, \beta_{S^{g}}$ measure the monthly average responses one month, ..., and $S^{g}$ months $\left(S^{1}=8, \ldots, S^{5}=12\right.$, where $g=\{1,2,3,4,5\}$ stands for the groups of treated workers) after the announcement, respectively. Since, as we mentioned, consumption is a flow variable and total debt balance is a stock variable in levels, we define the total effects within the pre and post windows differently. If the dependent variable is consumption, we define $c_{p r e}^{g} \equiv \sum_{t=-6}^{-1} \beta_{t}^{g}$ as the cumulative consumption response, whereas $d_{p r e}^{g} \equiv \frac{1}{6} \sum_{t=-6}^{-1} \beta_{t}^{g}$ stands for the average total debt response, both in the pre-policy window in group g. $c_{\text {announce }}^{g} \equiv \sum_{t=0}^{\tau^{g}-1} \beta_{t}^{g}$ is the cumulative consumption response within the announcement window, while $d_{\text {announce }}^{g} \equiv \frac{1}{\tau^{g}-1} \sum_{t=0}^{\tau^{g}-1} \beta_{t}^{g}$ is the average deleveraging effect (average total debt response) within the same window, where $\tau^{g}\left(\tau^{1}=3, \ldots, \tau^{5}=7\right.$ stands for months 2017:03,..., 2017:07) represents the initial months of withdrawals releases for groups $g=1, \ldots, g=5$, respectively. In the withdrawal window, $c_{\text {withdraw }}^{g} \equiv \sum_{t=\tau^{g}}^{S^{g}} \beta_{t}^{g}$ stands for the cummulative consumption response, whereas $d_{\text {withdraw }}^{g} \equiv \frac{1}{S^{g}-\left(\tau^{g}-1\right)} \sum_{t=\tau^{g}}^{S^{g}} \beta_{t}^{g}$, in turn, defines the average deleveraging effect in group $g$.

Finally, in the fourth specification we extend model (3) to assess heterogeneity in the consumption response to the withdrawals across groups of workers. We define $N$ as the number of subgroups of workers, which we decompose into subgroups $j_{1}, \ldots, j_{N}$, such that the $N$ th subgroup is absorved in the regression of the following model:

$$
\begin{aligned}
Y_{i, t}^{g} & =\sum_{s=-1}^{S^{g}} \beta_{s}^{g} \times \$ W_{i}^{g} \times 1_{\text {months }}+\sum_{s=-1}^{S^{g}} \beta_{j_{1}, s}^{g} \times 1_{j_{1}} \times \$ W_{i}^{g} \times 1_{\text {months }}+\ldots \\
& +\sum_{s=-1}^{S^{g}} \beta_{j_{(N-1)}, s}^{g} \times 1_{j_{(N-1)}} \times \$ W_{i}^{g} \times 1_{\text {month } s}+\alpha_{i}^{g}+\delta_{t}+\epsilon_{i, t} .
\end{aligned}
$$


Chapter 3. Follow the Money: The Effects of Liquidity Shocks on Consumption and Debt -

\section{4 \\ Results}

We present the results following the order of the models presented previously in the methodology section. First, we estimate the average response of consumption and total debt to the withdrawals program of the FGTS as in equation (1). Second, we split the post-policy period into the announcement period and the withdrawal period following equation (2). Then we assess dynamics using the distributed lag model (3). Lastly, we apply the model specification (4) to assess heterogeneity in the consumption response across workers. We present the main results both in the matched sample (after propensity score matching based on the nearest neighbor) and the full sample ${ }^{30}$ in the period range from seven months before to twelve months after the announcement of the program (2016:05-2017:12). To mitigate the challenge in statistical inference related to an unbalanced sample, we use the estimated propensity scores as regression weights in the full sample analysis.

\subsection{1 \\ Matched Sample Analysis}

\subsubsection{0}

\section{The average response of consumption and total debt}

Table 3.3 shows results on the average consumption response by applying equation (1) in panel $\mathrm{A}$ and equation (2) in panel $\mathrm{B}$ in the five groups of treated workers - columns (A) - (E). In panel A, the coefficients on $\$ W_{i}^{g} \times 1_{\text {pre }}$ capture the difference in consumption per $\$ 1$ of the withdrawal amount between the treatment and control clusters in the period before announcement (2016:05-2016:11) relative to the first month in our sample period (2016:05). Analogously, the coefficients on $\$ W_{i}^{g} \times 1_{\text {post }}$ measure the consumption response after the announcement ${ }^{31}$ relative to the first month in our sample period.

In Panel A of table 3.3, coefficient estimates on the pretreatment period variable, $\$ W_{i}^{g} \times 1_{\text {pre }}$, are both economically small and statistically insignificant in all five columns. For example, for each $\$ 1$ withdrawn by the treatment cluster in group 5, consumption is on average $\$ 0.003$ more than its control cluster in the window before the program announcement and is statiscally insignificant $(p$-value $=0.91)$. It suggests that before the withdrawals program of the FGTS

\footnotetext{
${ }^{30}$ We randomly draw approximatelly 8 percent of the full database.

${ }^{31}$ Remind that we set a 6 -month withdrawal window following the first month of the withdrawal release for each group. Thus, the post-treatment for all groups begins on 2016:12 and ends on 2017:08, 2017:09, 2017:10, 2017:11 and 2017:12, for groups 1,2,3,4 and 5, respecitively.
} 
Chapter 3. Follow the Money: The Effects of Liquidity Shocks on Consumption and Debt -

there are no differences in consumption spending patterns between the matched treatment and control clusters. It provides evidence supporting our differencein-diferences approach and then reinforeces that the difference in consumption after the announcement is indeed the treatment clusters' response to the liquidity-income shock. Overall, coefficient estimates on the post-treatment period variable, $\$ W_{i}^{g} \times 1_{\text {post }}$, are both statistically and economically significant and ranges from $\$ 0.029$, in group 4 , to $\$ 0.058$, in group 2 , per month for every $\$ 1$ of withdrawn resources from inactives accounts of workers who qualified for the FGTS program.

Remind that in section 2 we show evidence supporting that the withdrawals program of the FGTS in 2017 was unanticipated by formal workers in Brazil. The program was announced in December 2016, three months before withdrawals release of qualified workers in group 1, which enable us to investigate the announcement and withdrawals effects separately. In Panel B, consistently with Agarwal and Qian (2014) ${ }^{32}$, there is evidence that workers are taking advantage of consumer credit in facilitating the consumption response in the announcement period by "borrowing" from the future withdrawal money and started spending immediately upon announcement. Coefficient estimates on the announcement period variable, $\$ W_{i}^{g} \times 1_{\text {announce }}^{g}$, in groups 2 and 3 shows a $\$ 0.030$ and $\$ 0.031$ increase, respectively, per month for every $\$ 1$ of withdrawn resources. The withdrawal effect, in turn, is stronger and more precisely estimated, as we see the coefficient estimates on the withdrawal period variable, $\$ W_{i}^{g} \times 1_{\text {withdraw }}^{g}$, also in panels B across groups of treated workers. The effects range from $\$ 0.046$ in group 1 to $\$ 0.092$ in group 5 per month for every $\$ 1$ of withdrawn resources.

Table 3.4 shows that the withdrawals program of the FGTS also implied a deleveraging effect among treated workers. We see in Panel A that workers in the treatment cluster reduce their total debt balance by amounts ranging from $-\$ 0.037$ (not statistically significant) in group 3 to $-\$ 0.13$ in group 5 for every $\$ 1$ withdrawn in the program during the whole post-treatment window (coefficients on the post-treatment period variable $\$ W_{i}^{g} \times 1_{\text {post }}$ ). Remind from subsection 3.2 that for result's interpretation, consumption spending (flow) is the monthly average response within the respective windows, whereas for total

${ }^{32} \mathrm{We}$ shall see in the subsection on heterogeneity of modalities below that it is due to credit card spending. 
Chapter 3. Follow the Money: The Effects of Liquidity Shocks on Consumption and Debt -

debt balance (stock) it is the average within the whole respective windows ${ }^{33}$. Panel B suggests that, unlike the effect on consumption, overall there is no deleveraging antecipation to the withdrawal window - coefficients on the annoucement period, $\$ W_{i}^{g} \times 1_{\text {announce, }}^{g}$, are insignificant ${ }^{34}$. Workers in general wait for the release of their groups' withdrawals to deleverage on total debt. Therefore, as the coefficients on the withdrawal period, $\$ W_{i}^{g} \times 1_{\text {withdraw }}^{g}$, show, total debt balances of workers in the treatment cluster decline by (statistically significant) amounts ranging from $-\$ 0.068$ in group 3 to $-\$ 0.183$ in group 5 for every $\$ 1$ withdrawn in the program during the whole withdrawal window.

\subsubsection{0}

\section{The Dynamics of Consumption and Debt Responses}

Tables 5-6 Tables 3.5-3.6 report the average monthly response of consumption and total debt, respectively, to the withdrawals program of the FGTS following the distributed lag model in equation (3). Tables also exhibit the average monthly response in the pre-treatment period and in the post-treatment period, which we decompose into announcement and withdrawal windows, to gauge both the consumption and the deleveraging impact of the liquidityincome shock. The results can be interpreted as an event study, with month 0 being the time of the announcement and $\tau^{g}=\{3,4,5,6,7\}$ being the initial months of withdrawals releases for groups $g=\{1,2,3,4,5\}$, respectively. As mentioned before, $\left\{c_{\text {pre }}^{g}, c_{\text {announce }}^{g}, c_{\text {withdraw }}^{g}\right\}$ and $\left\{d_{\text {pre }}^{g}, d_{\text {announce }}^{g}, d_{\text {withdraw }}^{g}\right\}$ stand for the cumulative response, in the case of the dependent variable is consumption spending, or the average response if the dependent variable is total debt balance within the pre-policy, announcement and withdrawal windows, respectively ${ }^{35}$. For easy of visualization, in these tables we do not report the monthly coefficients within the pre-treatment window and we set the monthly

\footnotetext{
${ }^{33}$ As we mentioned in subsection 3.2 , total debt is a stock variable. We report results in levels instead of reporting them in levels change for convenience with model specifications 1 and 2. Debt levels changes are transitory, impacting in general only the first month of withdrawal release across groups. Thus, the The coefficient estimations of the effect of the withdrawals within the post-treatment windows do not show up significantly since the monthly significant effect averages with the remaining insignificant coefficient's months of these windows. By reporting the post-treatment windows' effects across groups using total debt in levels, results shows up significantly since the level of total debt declines persistently. The main difference between using either total debt in levels or in levels change refers to interpretation. The coefficient estimations of the effect of the withdrawals using the former should be taken as the monthly average within the post-treatment window, whereas the latter is the average effect within the whole post-treatment windows.

${ }^{34}$ One exception is group 5 , which shows a decline of $\$ 0.084$ for every $\$ 1$ withdrawn in the program. However, we will see in the full sample analysis that this announcement effect becomes economically and statistically insignificant.

${ }^{35}$ Note again that the dependent variable consumption spending is a flow variable, whereas the dependent variable total debt balance is a stock. For further details on interpretation see subsection 3.2 .
} 
Chapter 3. Follow the Money: The Effects of Liquidity Shocks on Consumption and Debt -

coefficients within the withdrawal windows in bold. Tables tables 5-6 Tables 3.5-3.6 confirm results in previous specifications and, for example, differences between in the cumulative (average) responses between treatment and control clusters in the pre-treatment window, $c_{p r e}^{g}\left(d_{p r e}^{g}\right)$, are widespread insignificant both economically and statistically.

Consistently with the regression results in table 3.3, the monthly coefficients of the consumption response within the withdrawal windows of each group are overall economically and statistically significant, whereas those within the announcement window are not so widespread significant. We see that $c_{\text {announce }}^{g}$ shows a $\$ 0.119$ and a $\$ 0.155$ cumulative response in groups 2 and 3 , respectively, for every $\$ 1$ withdrawn in the program. Remind that the time range of the annoucement window of each groups increase by one month for each group from group 1 to group 5, as shows the monthly coefficients not in bold in the table. Thus, one have to take this in mind when comparing $c_{\text {announce }}^{g}$ across groups if the dependent variable is consumption spending. $c_{\text {withdraw }}^{g}$, in turn, shows cumulative consumption responses within the withdrawal six-months windows ranging from $\$ 0.275$ in group 1 to $\$ 0.553$ in group 5. Table 3.4 reports the average monthly coefficients on the total debt response. It shows that the responses in general occur from the beginning the withdrawal windows ${ }^{36}$, concurrently with stronger consumption reponses. The average total debt responses within the withdrawal windows, $d_{\text {withdraw }}^{g}$, range from $-\$ 0.068$ in group 3 and $-\$ 0.183$ in group 5 .

\subsection{2}

\section{Full Sample Analysis}

We have performed the analysis so far on a smaller sample in which the treatment group and control group are matched on several demographics and credit variables. To ensure that the results can be generalized to the full sample, we repeat the main analysis on the full sample. As we mentioned, we consider all treated workers with some consumption and debt information in the SCR database. We exploit the estimated propensity scores by including them as regression weights in the full sample difference-in-differences analysis to address the challenge in statistical inference related to an unbalanced sample. Furthermore, by using all treated workers in the analysis, we are able to speak to the external validity of our results in the matched sample

\footnotetext{
${ }^{36}$ One exception is again group 5 , which shows an average decline of $\$ 0.084$ for every $\$ 1$ withdrawn in the program within the announcement window. However, we will see in the full sample analysis that this announcement effect becomes economically and statistically insignificant.
} 
Chapter 3. Follow the Money: The Effects of Liquidity Shocks on Consumption and Debt -

analysis. ${ }^{37}$ We overall see that results are more precisely estimated and stable across groups of treated workers relative to results estimated on the matched sample. Therefore, we include in this subsection more exercises related to heterogeneity of consumption and debt modalities and heterogeneity across consumers.

\subsubsection{0}

\section{The average response of consumption and total debt}

First, we analyze the average response of consumption and total debt. Tables 3.7 and 3.8 exhibts the full sample analogues of the consumption and debt responses on the matched sample in Tables 3.3 and 3.4, respectively, using model specifications 1 in Panel A and 2 in Panel B. Table 3.7 shows that coefficient estimates on consumption responses in the pretreatment period variable, $\$ W_{i}^{g} \times 1_{\text {pre }}$, are in general both economically small and statistically insignificant. One exception in group 3 , since the pre-treatment shows up statiscally significant. However, it should not rise much concern. It is due to only one month (2016:11) -the remaining five months of the pre-treatment window are both economically small and statistically insignificant - and we further note that the post-treatment coefficients are rather similar to the other groups' coefficients. In Panel A, coefficient estimates on the post-treatment period variable, $\$ W_{i}^{g} \times 1_{\text {post }}$, are both statistically and economically significant and ranges from $\$ 0.042$, in group 4 , to $\$ 0.054$, in group 2 , per month for every $\$ 1$ of withdrawn resources from inactives accounts of workers who qualified for the FGTS program. In Panel B, we see that the consumption antecipation shows up in all groups of treated workers, which reinforces that workers are taking advantage of consumer credit in facilitating the consumption response in the announcement period by "borrowing" from the future withdrawal money and started spending immediately upon announcement. Coefficient estimates on the announcement period variable, $\$ W_{i}^{g} \times 1_{\text {announce }}^{g}$, renges from $\$ 0.019$ in group 4 to $\$ 0.024$ in group 1 per month for every $\$ 1$ of withdrawn resources. The withdrawal effect, in turn, is stronger as we see the coefficient estimates on the withdrawal period variable, $\$ W_{i}^{g} \times 1_{\text {withdraw }}^{g}$, also in panels $\mathrm{B}$ across groups of treated workers. The effects range from $\$ 0.064$ in group 4 to $\$ 0.082$ in group 5 per month for every $\$ 1$ of withdrawn resources.

Table 3.8 exhibits the total debt response, as Table 3.4 on the matched sample. In Panel A, coefficient estimates on the post-treatment period variable,

\footnotetext{
${ }^{37}$ The standard errors reported for the full sample analysis do not take into account the estimation error associated with estimating the propensity score, and the reported errors may thus be downward biased, making estimates appear more precise than they actually are. However, we believe that this issue is lessened by the size of our full sample.
} 
Chapter 3. Follow the Money: The Effects of Liquidity Shocks on Consumption and Debt -

$\$ W_{i}^{g} \times 1_{\text {post }}$, are both statistically and economically significant and indicates a decline of total debt ranging from $-\$ 0.033$, in groups 3 and 4 , to $-\$ 0.058$, in group 2 for every $\$ 1$ of withdrawn resources from inactives accounts of workers who qualified for the FGTS program in the whole post-treatment window. In Panel B, coefficient estimates on the announcement period variable, $\$ W_{i}^{g} \times 1_{\text {announce }}^{g}$, indicates that the workers generally deleverage following the withdrawal windows. ${ }^{38}$ The withdrawal effect, $\$ W_{i}^{g} \times 1_{\text {withdraw }}^{g}$, also in panels $\mathrm{B}$ across groups of treated workers. The effects range from $-\$ 0.058$ in group 3 to $-\$ 0.089$ in group 5 for every $\$ 1$ of withdrawn resources during the whole withdrawal window.

\subsubsection{0}

\section{The Dynamics of Consumption and Debt Responses}

In addition to the average response analysis, we also apply the distributed lag model in equation (3) showing the dynamics of consumption and debt responses. Table 3.9 shows that the monthly coefficients of the consumption response within both the announcement and the withdrawal windows of each group are overall economically and statistically significant, whereas those within the announcement window in Table 3.5 that uses the matched sample are not so widespread significant. We see that $c_{\text {announce }}^{g}$ shows results ranging from $\$ 0.073$ and $\$ 0.15$ as cumulative responses in groups 1 and 5, respectively, for every $\$ 1$ withdrawn in the program. ${ }^{39} c_{\text {withdraw }}^{g}$, in turn, shows cumulative consumption responses within the withdrawal six-months windows ranging from $\$ 0.383$ in group 4 to $\$ 0.490$ in group 5. Table 3.10 reports the average monthly coefficients on the total debt response. It shows that the responses in general occur following the withdrawal windows ${ }^{40}$. The average total debt responses within the withdrawal windows, $d_{\text {withdraw }}^{g}$, range from $-\$ 0.058$ in group 3 and $-\$ 0.089$ in group 5 .

We include an additional exercise on the dynamics of consumption and debt responses applied on the full sample. We restrict the sample to individuals that holds simultaneasly short-term liquidity sources of both consumption

\footnotetext{
${ }^{38}$ One exception is group 2 , that show a coefficient of $-\$ 0.017$ for every $\$ 1$ of withdrawn resources from inactives accounts of workers who qualified for the FGTS program in the whole announcement window. We shall see below that it might be capturing an outlier regarding to payroll debt in 2016:11.

${ }^{39}$ Remind again that the time range of the annoucement window of each groups increase by one month for each group from group 1 to group 5 , as shows the monthly coefficients not in bold in the table. Thus, one have to take this in mind when comparing $c_{\text {announce }}^{g}$ across groups if the dependent variable is consumption spending

${ }^{40}$ One exception is group 2, which shows an average decline of $\$ 0.017$ for every $\$ 1$ withdrawn in the program within the announcement window. However, we shall see below in Table 3.12 that it likely reflects outliers exclusively in 2017:03 in group 2.
} 
Chapter 3. Follow the Money: The Effects of Liquidity Shocks on Consumption and Debt -

(credit card spending) and debt (overdraft debt). We additionally restrict the sample to individuals that also hold payroll debt, representing a relatively less liquid debt modality. The coefficient results in Table 3.11 shows that unlike in payroll debt, which shows overall no response within the announcement window, we see evidence that overdraft debt balance soars across groups of treated workers within the announcement window. In Table A.2 in Appendix A, we show the same exercise, but overdraft debt is replaced by credit card debt, which is another liquid debt modality. We also find some evidence that there are treated individuals who increase credit card debt during the announcement window in contrast with the negative debt response within the withdrawal window. This result is consistent with studies in the literatures on consumption effects of income and liquidity shocks (e.g. Agarwal and Qian (2014),Gelman et al. (2018)). That is, we show that financial incentives drive (constrained) ${ }^{41}$ consumers' spending behaviour not only through credit card spending. Interestingly, we also document a newly response mechanism that highlights consumers use multiple sources of short-term liquidity in debt modalities to smooth consumption following a positive unanticipated liquidity shock. Then, after the withdrawals, consumers start to deleverage the increased short-term debt modalities and switch to (cheaper) credit card spending ${ }^{42}$, as we observe a stronger spending response within the withdrawals window.

\subsubsection{0}

\section{Response heterogeneity by consumption categories and debt modalities}

We next study the dynamics of heterogeneous responses to the withdrawals program across different modalities following specification 1 . The specialized literature documents heterogeneity in the type of spending response to positive income shocks (e.g.,Parker et al. (2011),Agarwal and Qian (2014)). In ou data, consumption is disaggregated into credit card spending and vehicles and real estate down payments. There are several types of debt modalities comprising total debt. As mentioned, we will highlight three modalities (credit card debt, overdraft debt and payroll debt) that account for more than 60 percent of total debt. We also include in the analysis the amount of total debt that are overdue and as well as default debt. Since we have shown that there is no pre-treatment effect in Tables $7-8$ Tables 3.7 and 3.8 , we absorb the pre-treatment variable $\$ W_{i}^{g} \times 1_{\text {pre }}$ in the heterogeneity analysis to increase

\footnotetext{
${ }^{41}$ In a next version of this paper, we will have data on liquid assets which will enable us to study more precisely that these results are driven by liquidity constraints.

${ }^{42}$ Agarwal and Qian (2014) show an even stronger increase of debit card spending with cash in hand, which is a cheaper modality relative to credit card spending.
} 
Chapter 3. Follow the Money: The Effects of Liquidity Shocks on Consumption and Debt -

power and for the purpose of easier interpretation. ${ }^{43}$ Coefficient estimates on the post-treatment period variable, $\$ W_{i}^{g} \times 1_{\text {post }}$, are both statistically and economically significant and rather stable across groups of treated workers, which is a gauge of robustness of the results applied on the full sample.

To summarize, Table 3.12 shows that most of the consumption response to the withdrawals program stems from credit card spending. It indicates a rise of spending ranging from $\$ 0.030$ in group 3 and $\$ 0.033$ in group 5 in average per month in the post-treatment window for every $\$ 1$ of withdrawn resources from inactives accounts of workers who qualified for the FGTS program. It accounts for approximately 70 percent of the consumption response, whereas real estate down payments accounts for 26 percent and vehicles down payments accounts for the remaining 4 percent $^{44}$. This result is consistent with the literature. Agarwal and Qian (2014) estimate a coefficient on $\$ W_{i}^{g} \times 1_{\text {post }}$ of $\$ 0.053$ as monthly average credit spending in the post-treatment window for every $\$ 1$ of disbursement resources in a policy experiment in Singapore. One has to keep in mind that credit card spending in Singapore accounts for 30 percent of the aggregate personal consumption, while in Brazil close to 20 percent of aggregate household consumption expenditure in the country is purchased using credit card. ${ }^{45}$ Furthermore, also consistently with some prior research (e.g Aaronson et al. (2012),Parker et al. (2011)), we find positive response in big-ticket durables, especially in real estate down payments. ${ }^{46}$

${ }^{43}$ We follow Agarwal and Qian (2014) in this procedure. We have verified that our results are qualitatively the same by adding the pre-treatment months in the analysis in all types of consumption and all debt modalities across groups of treated workers in the heterogeneity analysis. Moreover, pre-treatment periods are overall insignificant.

${ }^{44}$ It is a back-of-the-envelope calculation of the ratio of the average responses of each category (weighted by groups' observations) to the average response of consumption within the post-treatment window.

${ }^{45}$ It is according to the Brazilian Association of Credit Card and Service Companies (ABECS) and the National Accounts System - excluding imputed rent for dwelling services - from the Brazilian Institute of Geography and Statistics (IBGE) in 2017. Agarwal et al. (2007) find similar results for the U.S, where credit card spending accounts for 20 percent of the aggregate personal consumption during the late 90's ( Chimerine and Incorporated (1997)

${ }^{46}$ Notwithstanding, real estate credit contracts are very infrequent, which reduces the size of the response due to a large number of individuals that do not hold this type of contracts (many zeros by considering the full sample). By restricting the sample for individuals that made real estate down payments (and that eventually hold any other consumption category or debt modality), the size of the coefficient response can increase to up to $\$ 0.60$ in average per month in the post-treatment window for every $\$ 1$ of withdrawn resources from inactives accounts. Note that it imples an accumulated response of more than 100 percent within the post-treatment window among individuals that buy a house, which is a common results for very large purchases. Similarly, payroll debt, which is also a relatively less frequent debt modality, restricting the sample for individuals that hold payroll debt (and that eventually hold any other consumption category or debt modality), the average deleveraging response within the whole post-treatment window rises to approximately $-\$ 0.15$ (near $-\$ 0.02$ on the full sample). 
Chapter 3. Follow the Money: The Effects of Liquidity Shocks on Consumption and Debt -

This finding is suggestive of an important role for liquidity constraints, as the unanticipated shock may have provided otherwise unavailable down payments for debt-financed purchases of cars and dwelling.

Table 3.12 also exhibts the response of debt modalities. Payroll debt stand out with 48 percent of total debt response by considering an average response of nearly $-\$ 0.020$ across groups in the post-treatment window for every $\$ 1$ of withdrawn resources ${ }^{47}$. Credit card debt and overdraft debt show similar responses accounting for 15 percent and 13 percent of total debt response, respectively. Furthermore, by considering the average response in the posttreatment window of total overdue debt and total default debt, we highlight that around 24 percent of the deleveraging response on total debt refers to overdue debt and 16 percent relates to default debt.

Nevertheless, to move from the consumption and debt responses of each group of treated workers to aggregate consumption and debt responses, we need to take a stand on total responses in order to gauge the overall impact of the program stimulus. For illustrative purposes, we report some back-of-theenvelope calculations along these lines, in which we consider the statistically significant responses in each group in tables 7 and 8 Tables 3.7 and 3.8 as well as consider the group's average withdrawn amounts. Thus, the implied consumption and total debt response of treated workers in our SCR full sample represent, respectively, nearly 53 percent and 7 percent of workers' average withdrawals resources in the program. Considering the program totaled US $\$ 13.6$ billion and that around 64 percent of it belongs to treated workers with some information in the SCR in our full sample, the direct (partial equilibrium) impact of the withdrawals program in rising treated workers' consumption and reducing treated workers' total debt represents approximately 35 percent and 5 percent, respectively, of total resources in the withdrawals program of the FGTS. We are not able to keep track of the remaining 26 percent of total resources in the withdrawals program in our full sample as we do not have data on cash/check spending, debt card spending and savings. Moreover, as we mentioned, nearly $1 / 3$ of the total resources in the withdrawals program are not in the SCR database.

\subsubsection{0}

\section{Heterogeneity of consumption response across consumers}

Now we study the dynamics of heterogenous responses to the withdrawal program across different treated workers. Previous literature has demonstrated

${ }^{47}$ Again we consider a simple calculation of the ratio of the average responses of each debt modality (weighted by groups' observations) to the average response of total debt within the post-treatment window. 
Chapter 3. Follow the Money: The Effects of Liquidity Shocks on Consumption and Debt -

that constrained consumers respond more strongly to positive shocks (e.g., Agarwal and Qian (2014); Johnson et al, 2006). The empirical literature emphasizes that some observed characteristics such as income, debt, and liquid wealth are likely to bear some correlation with the unobserved characteristics that may trigger a violation of the permanent income hypothesis. Earlier studies use demographics as proxies for liquidity constrained consumers. For instance, prior papers argue that young and old consumers are more likely to be liquidity constrained. To this respect, we show an initial approach to heterogeneity across consumers. We follow previous literature to argue that young and old workers are more likely to constrained consumers. We estimate each group's response to the stimulus in one regression using specification in equation (4). Our data allow us to understand differences in the full path of the workers' consumption responses across different debt modalities. However, we leave a more complete assessment of different demographic proxies, asset and debt modalities holdings for the assessment of constrained consumers for future investigation.

Given that in previous tables in general no pretreatment effect was shown, as in the modalities heterogeneity analysis, we absorb the pretreatment variable $\left(\$ W_{i}^{g} \times 1_{\text {pre }}\right)$ to increase power ${ }^{48}$ and for easy of interpretation. We classify the sample of workers according to their age in the four months before our analysis sample (i.e., 2016:01-2016:04): workers with low level of age if his of her age is below the tenth percentile of the distribution, or equivalently to around 26 years old across groups of treated workers, and workers with high level of age if his or her age is above the top decile of the distribution, or equivalently to 54 years old. Therefore, for example, if there are two groups, we estimate each group's response to the shock in one regression using the specification in equation (4), with group $N=2$ being absorbed. We then plot, for each group of consumers, the the cumulative consumption coefficients (starting from the announcement month $s=0$ ) up to each month in the post-treatment period in the x-axis, $c_{t^{g}} \equiv \sum_{s=0}^{t^{g}} \beta_{s}^{g}$, where $t^{g}=\left\{0, \ldots, S^{g}\right\}$, along with their corresponding 95 percent confidence intervals in Figure 7 Figure 3.7.

Young individuals typically have low liquid wealth and high income growth, they are disproportionately likely to be liquidity constrained (e.g., Jappelli (1990),Jappelli et al. (1998)). There is also evidence that older individuals increase their spending on receiving (predictable) pension checks

\footnotetext{
${ }^{48}$ To include the pre-treatment month does not change qualitatively the results and it exhibits parallel trends in the pretreatment period in all subgroups in the heterogeneous analysis.
} 
Chapter 3. Follow the Money: The Effects of Liquidity Shocks on Consumption and Debt -

(Wilcox (1989),Stephens (2002)). Outside the null PIH hypothesis of $\beta_{s}^{g}=0^{49}$, older households might also spend relatively more because they have shorter time horizons on average.

Keeping in mind the degree of statistical significance, visual inspection of Figure 7 Figure 3.7 shows that the extreme groups according to age have qualitatively different average consumption response in the post-treatment period. We see evidence that both young and old treated workers spend more within the withdrawal window. Interestingly, within the announcement window young workers spend up to $\$ 19$ less for every $\$ 100$ withdrawn in the program ${ }^{50}$, which suggests that relatively more young workers might be liquidity constrained in accessing multiple sources of short-term liquidity to smooth consumption.

\subsection{3}

\section{Robustness}

In this section we assess robustness of the statistical inference that conduct a cross sectional test as suggested by the Bertrand et al. (2004). We compute the average monthly consumption and total debt during the six months before treatment (2016:06-2016:11), and during the months after treatment (2016:12-2017:08,.., 2016:12-2017:12, respectively, for each of the five groups of treated workers). Then we compute, as our dependent variables, the difference between the after-treatment average and before-treatment average for each individual. We then regress the dependent variables on the withdrawn amount of each group $g, \$ W^{g}$. In Table A.3 in Appendix A, in Panels A and $\mathrm{B}$, we report results on consumption in the matched sample and in the full sample, respetively. Panels $\mathrm{C}$ and $\mathrm{D}$ similarly show the results on total debt.

\footnotetext{
${ }^{49}$ The Euler-equation literature focuses on testing whether predictable changes in income are orthogonal to the residual $\left(u_{i, t+1}\right)$ over time; that is, whether $\beta_{s}^{g}$ equals zero (Chamberlain 1984; Souleles 2004). In contrast, here we use the exogeneous defined timing of withdrawal allowance and a difference-in-differences identification to ensure orthogonality between the residual and our withdrawal regressor in the cross-section, which allows us to estimate $\beta_{s}^{g}$ and, thus, measure the causal effect of the withdrawals on expenditure, regardless of whether the PIH is true or not. Nonetheless, our estimate still provides a direct test of the PIH, which implies that households should consume at most the annuitized value of a transitory increase in income, and Ricardian equivalence, which implies no spending response at all.

${ }^{50}$ We run F-tests of the difference in the cumulative coefficients between the young and old groups within the announcement windows, $c_{\text {announce }}^{g} \equiv \sum_{t=0}^{\tau^{g}-1} \beta_{t}^{g}$, across groups and the results are statistically significant in groups 2 and 4 of treated workers $(p$-value $<$ $0.001)$. In group $5 p$-value $=0.11$ and in groups 1 and $3 p$-value $=0.39$ and $p$-value $=$ 0.27 , respecively. We also run F-tests in the cumulative coefficients between the young and old groups within all post-treatment windows $c_{\text {post }}^{g} \equiv \sum_{s=0}^{S^{g}} \beta_{s}^{g}$ and the results are overall statistically insignificant, which suggests we cannot reject that young and old consumption behaviour following the liquidity shock is different.
} 
Chapter 3. Follow the Money: The Effects of Liquidity Shocks on Consumption and Debt -

We claim that our main findings remain in the tests.

\section{5}

\section{Conclusion}

This study uses an unique panel dataset of consumption and debt information for more than 12 million workers in Brazil to analyze how consumption and debt responded to an unanticipated fiscal stimulus program announced on December 22, 2016. The unique policy experiment by the Brazilian government allows us to distinguish an announcement effect and a withdrawal effect. We apply a difference-in-differences identification to estimate the response to the program. Workers that have inacctive accounts of the FGTS created after December 31, 2015 or whose inactive accounts were cleaned up by withdrawals before the program announcement were not elegible for the withdrawals program of the FGTS in 2017. We use this exclusion restriction to identify the causal effect of the program on consumption and debt by using these workers as our control clusters for each group of the five groups of treated workers defined by the program timeline of withdrawals.

We find that consumption rose following the announcement of the program: for each dollar withdrawn from a inactive account of the FGTS, workers on average accumulated a rise of $\$ 0.53-25$ percent of which particularly within the announcement window, which is a stong anticipation effect. Consumption rose primarily in credit card spending after the program announcement (on average 70 percent of the consumption response) followed by the increase of big-ticket durables, specially real estate down payments (on average 26 percent of the consumption response), which is suggestive of an important role for liquidity constraints, as the unanticipated shock may have provided otherwise unavailable down payments for debt-financed purchases of durables. Furthermore, consumption response was heterogeneous across individuals. Constrained consumers, measured as young or old, showed stronger consumption responses. The withdrawals program of the FGTS also implied a deleveraging effect after the withdrawals releases. Treated workers on average reduced total debt by $\$ 0.07$ for each $\$ 1$ withdrawn. In addition, payroll debt stood out among modalities, accounting for nearly 48 percent of total debt response after the program announcement.

Our main contributions to prior literature follow. First, our database allows us to study both unanticipated and anticipated effects to liquidityincome shocks and to attest consistency with life-cycle theory predictions. Very few papers are able to do so mainly due to lack of cross-sectional variation or due to the difficulty in identifying shocks that are geneuinely exogeneous and 
Chapter 3. Follow the Money: The Effects of Liquidity Shocks on Consumption and Debt -

unanticipated. Second, within the announcement window indebted consumers used short-term liquidity in debt modalities (overdraft debt and credit card debt) in addition to credit card spending to smooth consumption, which is mostly overlooked in the literature on methods of smoothing consumption. It implies that prior work based on micro-data with one single debt type (e.g., credit card debt) likely underestimates the the debt response to income shocks. To this respect, our database and this policy experiment allows us to study how consumption/savings behaviour adjust to a shock more comprehensively.

We should highlight that the results we report so far on the matched sample should be taken with caution. Responses across groups in several cases are not very stable, as one would expect after controlling in the regressions for individual fixed effects and seasonal and other concurrent aggregate factors. As Figure 3 Figure 3.3 on the distrubution of monthly income suggests, there is a mass of poorer workers in the treatment cluster that does not exist in the control cluster. More importantly, since income is a relevant covariate in the logistic regression in the propensity score matching, it affects the quality of the characteristics' overlapping between treatment and control clusters. Since poorer workers are likely to be constrained, they probably account for a large share of the consumption variation following the shock that is discarded by the nearest neighbour algorithm. In the full sample analysis, since we use the individual propensity scores as weights in the regression, we include these otherwise excluded individuals in the sample to deal with challenges related to unbalanced sample. Nevertheless, the estimates in the matched sample analysis may be more sensitive to outliers and may be causing bias due to unobservable heterogeneity with respect to consumption/savings behaviour. We leave for future endeavors to improve the matching sample by including more information on the logistic regression so that it enhances the overlapping of characteristics of the treatment and control clusters, as well as to test alternative matching algorithms.

Other future improvements refer to heterogeneity of consumption responses across consumers. Our database allows to further explore consumption differences by employment status. We will soon merge our database to assets issued by all banks in the country. This will be an important source to expand the scope of use of the withdrawals resources, specially among unconstrained workers. Furthermore, a growing body of research has identified individual balance sheets as important channels through which financial shocks to individuals can be amplified, with higher debt-to-asset ratios leading to higher implied elasticities of consumption with respect to wealth. We could further explore it by debt modalities. 
Chapter 3. Follow the Money: The Effects of Liquidity Shocks on Consumption and Debt -

We mention at least more two possible extensions. First, it would be of interest to consider likely unfolded household effects of the withdrawals programs to study, for example, household risk sharing. Second, we can explore variation through geographic heterogeneity of responses to assess whether they are different in booming and recessive local economies, which is gap in the empirical literature based on methodologies that measure (direct) partial equilibrium effects of income shocks conditional to the state of economy in the aggregate.

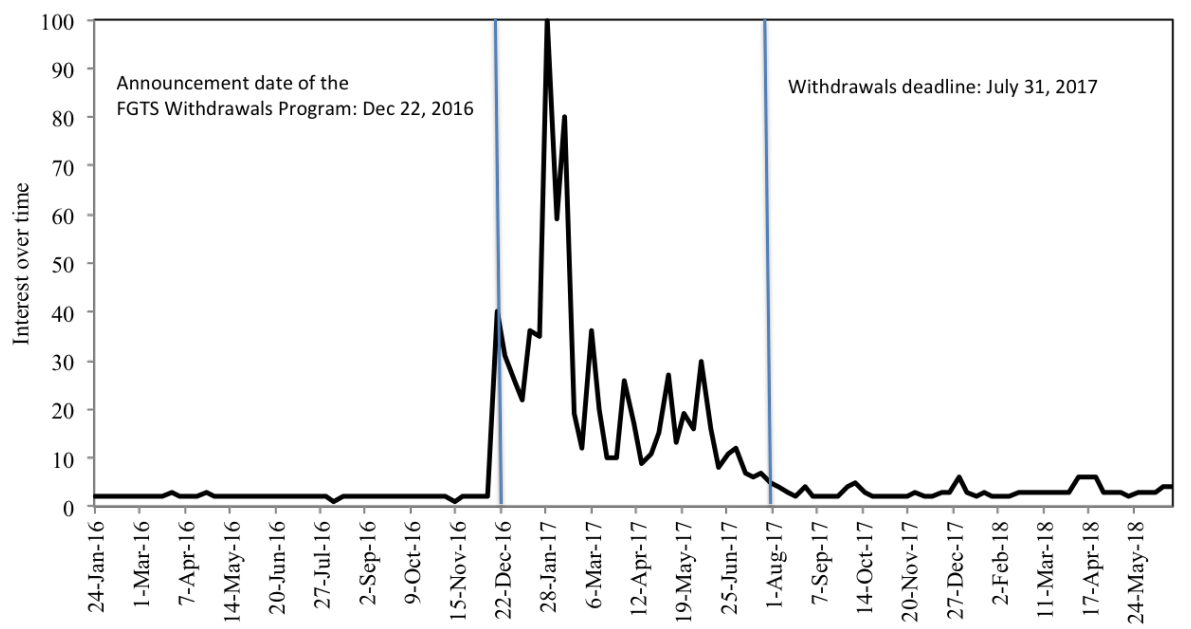

Figure 3.1: Web Searches for "FGTS saques" on Google Trends

Notes: This figure depicts a web search on Google Trends for "FGTS saque" ("FGTS withdrawal" in english) in all categories of websites in Brazil in a 30-month period between Jan 24, 2016 an Jun, 24 2018. As the website states, numbers represent search interest relative to the highest point on the chart for the given region and time. A value of 100 is the peak popularity for the term. A score of 0 means there was not enough data for this term. 
Chapter 3. Follow the Money: The Effects of Liquidity Shocks on Consumption and Debt -

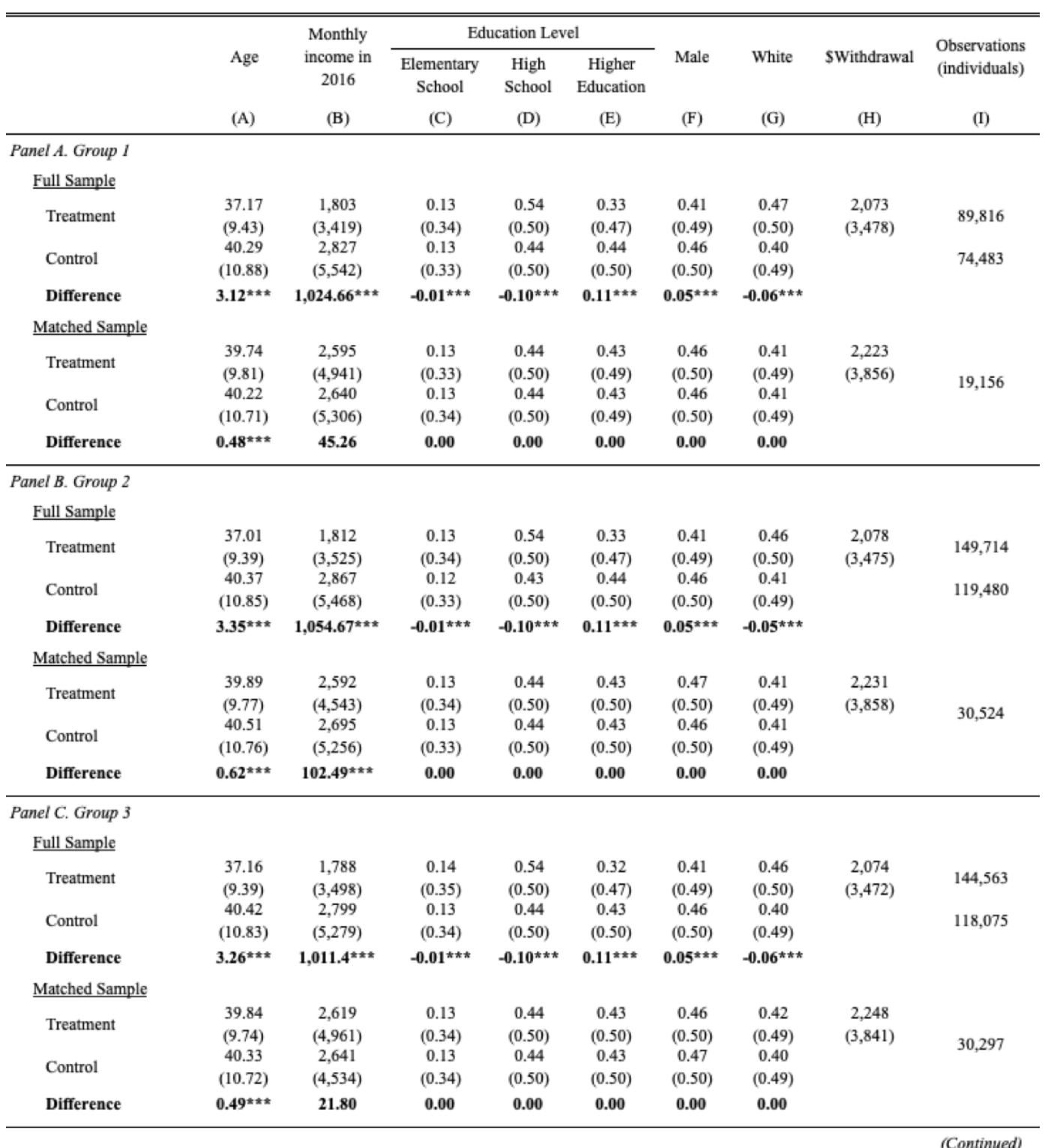

Table 3.1: Summary Statistics - Demographics 
Chapter 3. Follow the Money: The Effects of Liquidity Shocks on Consumption and Debt -

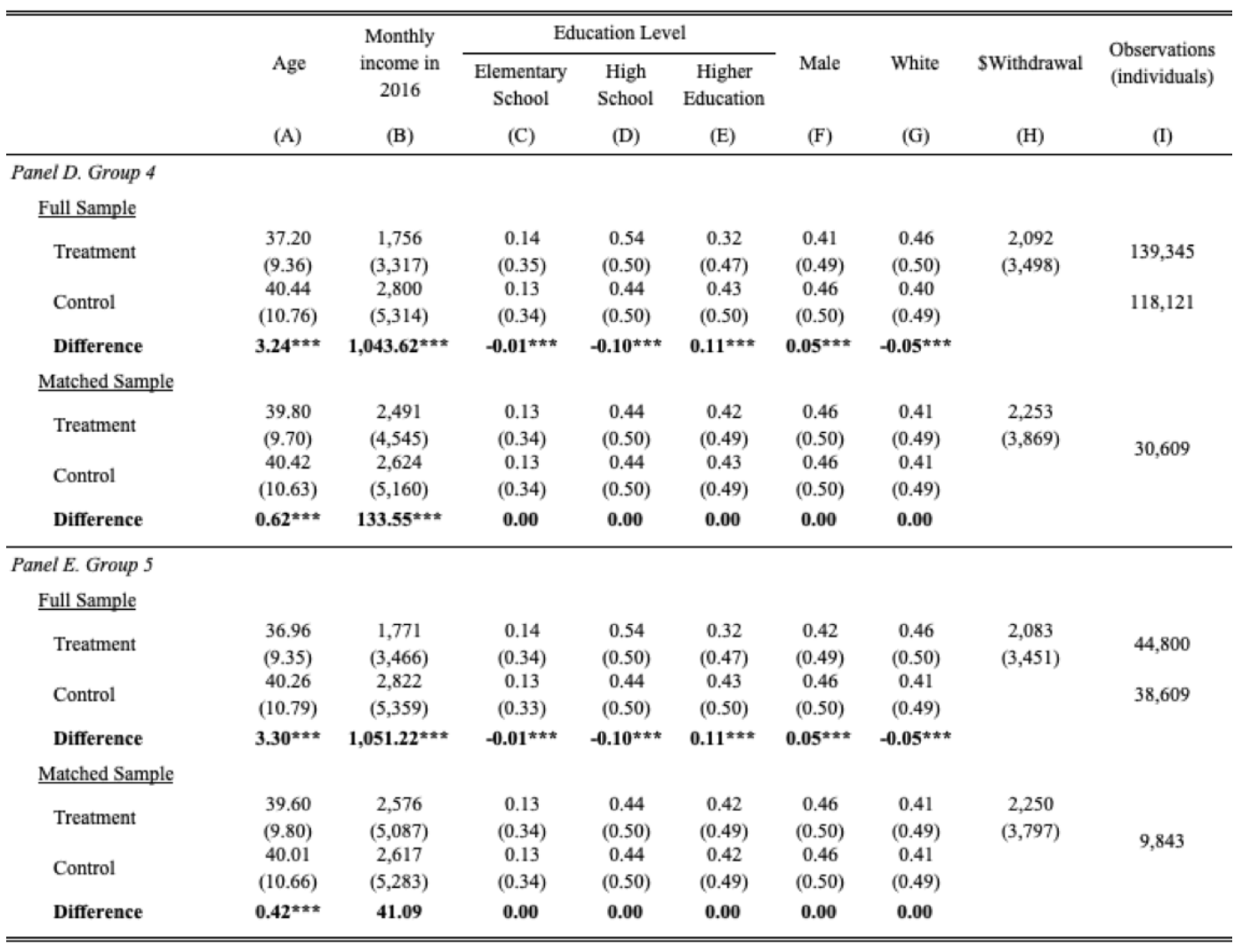

\section{Summary Statistics - Demographics (Continued)}

Notes: This table reports the summary of demographics statistics of our treatment and control sample both before and after propensity score matching (based on the nearest neighbor). The treatment sample consists of workers who qualify for the Withdrawal Program of the FGTS in 2017 (workers who had an inactive account of the FGTS until December, 31 2015) and the control sample consists of all workers who did not qualified for the program (individuals with FGTS accounts created after December 31, 2015 or with accounts cleared by withdrawals made before the program announcement). We exclude individuals with no information in the SCR database in our period sample (2016:05-2017:12). It exhibits the comparison of demographics between the treatment and control clusters for the five groups of treated workers. 
Chapter 3. Follow the Money: The Effects of Liquidity Shocks on Consumption and Debt -

\begin{tabular}{|c|c|c|c|c|c|c|c|c|c|c|c|}
\hline & \multirow{2}{*}{\multicolumn{4}{|c|}{ Consumption Proxies }} & \multicolumn{6}{|c|}{ Debt } & \multirow{3}{*}{$\begin{array}{c}\text { Observations } \\
\text { (Individual- } \\
\text { month) } \\
\text { (K) }\end{array}$} \\
\hline & & & & & \multirow[b]{2}{*}{$\begin{array}{l}\text { Total } \\
\text { Debt }\end{array}$} & \multirow[b]{2}{*}{$\begin{array}{c}\text { Total } \\
\text { Overdue }\end{array}$} & \multirow[b]{2}{*}{$\begin{array}{c}\text { Total } \\
\text { Default }\end{array}$} & \multicolumn{3}{|c|}{ Selected Debt Modalities } & \\
\hline & $\begin{array}{c}\text { Total } \\
\text { Consumption } \\
\text { (A) } \\
\end{array}$ & $\begin{array}{l}\text { Credit Card } \\
\text { Spending } \\
\text { (B) }\end{array}$ & $\begin{array}{l}\text { Vehicles } \\
\text { Down } \\
\text { Payments } \\
\text { (C) }\end{array}$ & $\begin{array}{l}\text { Real estate } \\
\text { Down } \\
\text { Payments } \\
\text { (D) }\end{array}$ & & & & $\begin{array}{l}\text { Credit Card } \\
\text { (F) }\end{array}$ & $\begin{array}{c}\text { Overdraft } \\
\text { (G) }\end{array}$ & $\begin{array}{l}\text { Payroll } \\
\text { (H) }\end{array}$ & \\
\hline \multicolumn{12}{|l|}{ Panel A. Group I } \\
\hline \multicolumn{12}{|l|}{ Full Sample } \\
\hline Treatment & $\begin{array}{c}3,067 \\
(6,974)\end{array}$ & $\begin{array}{c}2,886 \\
(5,134)\end{array}$ & $\begin{array}{c}4,063 \\
(14,158)\end{array}$ & $\begin{array}{c}23,633 \\
(59,397)\end{array}$ & $\begin{array}{c}7,986 \\
(16,093)\end{array}$ & $\begin{array}{c}2,648 \\
(6,860)\end{array}$ & $\begin{array}{c}3,572 \\
(8,099)\end{array}$ & $\begin{array}{c}1,165 \\
(3,170)\end{array}$ & $\begin{array}{c}1,080 \\
(2,589)\end{array}$ & $\begin{array}{c}13,332 \\
(17,874)\end{array}$ & $1,243,117$ \\
\hline Control & $\begin{array}{c}4,347 \\
(10,165)\end{array}$ & $\begin{array}{c}4,011 \\
(6,941)\end{array}$ & $\begin{array}{c}5,528 \\
(16,315)\end{array}$ & $\begin{array}{c}26,637 \\
(69,048)\end{array}$ & $\begin{array}{c}13,098 \\
(23,033)\end{array}$ & $\begin{array}{c}2,781 \\
(7,220)\end{array}$ & $\begin{array}{c}3,891 \\
(8,682)\end{array}$ & $\begin{array}{c}1,346 \\
(3,222)\end{array}$ & $\begin{array}{c}1,193 \\
(2,847)\end{array}$ & $\begin{array}{c}19,799 \\
(22,937)\end{array}$ & $1,106,019$ \\
\hline Difference & $1,279.67^{* * *}$ & $1,124.98^{* * *}$ & $1,465.09^{* * *}$ & $3,004.31$ & $5,112.26^{* * *}$ & $132.9^{* * *}$ & $318.89^{* * *}$ & $181.05^{* * *}$ & $112.86^{* * *}$ & $6,466.36^{* \pm *}$ & \\
\hline \multicolumn{12}{|c|}{ Matched Sample } \\
\hline Treatment & $\begin{array}{c}3,922 \\
(9,367)\end{array}$ & $\begin{array}{c}3,603 \\
(6,124)\end{array}$ & $\begin{array}{c}4,752 \\
(13,631)\end{array}$ & $\begin{array}{c}24,739 \\
(65,748)\end{array}$ & $\begin{array}{c}11,662 \\
(21,484)\end{array}$ & $\begin{array}{c}2,885 \\
(6,908)\end{array}$ & $\begin{array}{c}3,951 \\
(8,017)\end{array}$ & $\begin{array}{c}1,284 \\
(3,259)\end{array}$ & $\begin{array}{c}1,171 \\
(2,727)\end{array}$ & $\begin{array}{c}17,154 \\
(21,806)\end{array}$ & 288,794 \\
\hline Control & $\begin{array}{c}4,181 \\
(9,444)\end{array}$ & $\begin{array}{c}3,859 \\
(6,636)\end{array}$ & $\begin{array}{c}5,499 \\
(15,004)\end{array}$ & $\begin{array}{c}25,774 \\
(62,199)\end{array}$ & $\begin{array}{c}12,441 \\
(22,091)\end{array}$ & $\begin{array}{l}2,843 \\
(7,95)\end{array}$ & $\begin{array}{c}3,993 \\
(9,980)\end{array}$ & $\begin{array}{c}1,348 \\
(3,269)\end{array}$ & $\begin{array}{c}1,192 \\
(2,644)\end{array}$ & $\begin{array}{c}18,689 \\
(21,900)\end{array}$ & 281,689 \\
\hline Difference & $258.95^{* * *}$ & $255.47^{* * *}$ & 746.34 & $1,035.15$ & $778.62^{* * *}$ & -42.25 & 41.22 & $63.73^{\star \star *}$ & 21.18 & $1,534.53^{* \pm *}$ & \\
\hline \multicolumn{12}{|l|}{ Panel B. Group 2} \\
\hline \multicolumn{12}{|l|}{ Full Sample } \\
\hline Treatment & $\begin{array}{c}3,046 \\
(6,988)\end{array}$ & $\begin{array}{c}2,862 \\
(4,985)\end{array}$ & $\begin{array}{c}4,044 \\
(14,627)\end{array}$ & $\begin{array}{c}23,886 \\
(59,573)\end{array}$ & $\begin{array}{c}8,055 \\
(16,22)\end{array}$ & $\begin{array}{c}2,663 \\
(7,079)\end{array}$ & $\begin{array}{c}3,596 \\
(8,517)\end{array}$ & $\begin{array}{c}1,196 \\
(3,351)\end{array}$ & $\begin{array}{c}1,072 \\
(2,594)\end{array}$ & $\begin{array}{c}13,356 \\
(17,340)\end{array}$ & $2,081,561$ \\
\hline Control & $\begin{array}{c}4,349 \\
(11,243)\end{array}$ & $\begin{array}{c}4,019 \\
(6,831)\end{array}$ & $\begin{array}{c}5,259 \\
(15,842)\end{array}$ & $\begin{array}{c}25,145 \\
(85,226)\end{array}$ & $\begin{array}{c}13,492 \\
(23,763)\end{array}$ & $\begin{array}{c}2,848 \\
(8,593)\end{array}$ & $\begin{array}{c}4,059 \\
(11,034)\end{array}$ & $\begin{array}{c}1,381 \\
(3,525)\end{array}$ & $\begin{array}{c}1,231 \\
(2,994)\end{array}$ & $\begin{array}{c}20,242 \\
(23,226)\end{array}$ & $1,780,093$ \\
\hline Difference & $1,302.53^{* * *}$ & $1,156.94^{* * *}$ & $1,214.14^{* * *}$ & $1,259.6$ & $5,436.33^{* * *}$ & $184.75^{* * *}$ & $462.55^{* * *}$ & $185.05^{\star * *}$ & $158.38^{* * *}$ & $6,886.03^{* * *}$ & \\
\hline \multicolumn{12}{|c|}{ Matched Sample } \\
\hline Treatment & $\begin{array}{c}3,903 \\
(8,977)\end{array}$ & $\begin{array}{c}3,629 \\
(6,319)\end{array}$ & $\begin{array}{c}4,415 \\
(15,551)\end{array}$ & $\begin{array}{c}22,812 \\
(58,921)\end{array}$ & $\begin{array}{c}11,808 \\
(21,459)\end{array}$ & $\begin{array}{c}2,805 \\
(6,857)\end{array}$ & $\begin{array}{c}3,898 \\
(8,215)\end{array}$ & $\begin{array}{c}1,297 \\
(3,373)\end{array}$ & $\begin{array}{c}1,208 \\
(3,021)\end{array}$ & $\begin{array}{c}17,348 \\
(21,031)\end{array}$ & 462,485 \\
\hline Control & $\begin{array}{c}4,207 \\
(9,138)\end{array}$ & $\begin{array}{c}3,916 \\
(6,882)\end{array}$ & $\begin{array}{c}4,901 \\
(16,720)\end{array}$ & $\begin{array}{c}22,324 \\
(51,145)\end{array}$ & $\begin{array}{c}12,589 \\
(22,242)\end{array}$ & $\begin{array}{c}2,723 \\
(6,866)\end{array}$ & $\begin{array}{c}4,007 \\
(8,553)\end{array}$ & $\begin{array}{c}1,352 \\
(3,440)\end{array}$ & $\begin{array}{c}1,185 \\
(2,774)\end{array}$ & $\begin{array}{c}18,752 \\
(21,811)\end{array}$ & 450,742 \\
\hline Difference & $303.96^{* \pm *}$ & $287.20^{ \pm * *}$ & 486.23 & -488.06 & $781.32^{* * * *}$ & -81.91 & 108.57 & $55.76^{* \pm *}$ & -22.71 & $1,403.98^{* * *}$ & \\
\hline \multicolumn{12}{|l|}{ Panel C. Group 3} \\
\hline \multicolumn{12}{|l|}{ Full Sample } \\
\hline Treatment & $\begin{array}{c}3,012 \\
(6,785)\end{array}$ & $\begin{array}{c}2,830 \\
(5,053)\end{array}$ & $\begin{array}{c}3,914 \\
(11,496)\end{array}$ & $\begin{array}{c}23,268 \\
(58,119)\end{array}$ & $\begin{array}{c}7,980 \\
(15,900)\end{array}$ & $\begin{array}{c}2,584 \\
(7,052)\end{array}$ & $\begin{array}{c}3,427 \\
(7,790)\end{array}$ & $\begin{array}{c}1,182 \\
(3,100)\end{array}$ & $\begin{array}{c}1,050 \\
(2,414)\end{array}$ & $\begin{array}{c}13,253 \\
(17,496)\end{array}$ & $2,009,633$ \\
\hline Control & $\begin{array}{c}4,297 \\
(9,795)\end{array}$ & $\begin{array}{c}3,967 \\
(6,770)\end{array}$ & $\begin{array}{c}5,208 \\
(15,692)\end{array}$ & $\begin{array}{c}24,997 \\
(64,105)\end{array}$ & $\begin{array}{c}13,229 \\
(23,344)\end{array}$ & $\begin{array}{c}2,728 \\
(7,070)\end{array}$ & $\begin{array}{c}3,842 \\
(8,529)\end{array}$ & $\begin{array}{c}1,377 \\
(3,371)\end{array}$ & $\begin{array}{c}1,215 \\
(2,986)\end{array}$ & $\begin{array}{c}19,947 \\
(22,962)\end{array}$ & $1,759,676$ \\
\hline Difference & $1,285.8^{* * *}$ & $1,136.52^{\star \star \star \star}$ & $1,293.66^{* \star * *}$ & $1,728.37$ & $5,248.77^{* * *}$ & $143.58^{* \star * \star}$ & $414.8^{* * *}$ & $194.61^{* * *}$ & $164.3^{* * *}$ & $6,694.14^{* * *}$ & \\
\hline \multicolumn{12}{|c|}{ Matched Sample } \\
\hline Treatment & $\begin{array}{c}3,948 \\
(9,533)\end{array}$ & $\begin{array}{c}3,636 \\
(6,408)\end{array}$ & $\begin{array}{c}4,647 \\
(13,747)\end{array}$ & $\begin{array}{c}24,555 \\
(65,262)\end{array}$ & $\begin{array}{c}11,981 \\
(21,724)\end{array}$ & $\begin{array}{c}2,749 \\
(8,962)\end{array}$ & $\begin{array}{c}3,669 \\
(7,798)\end{array}$ & $\begin{array}{c}1,265 \\
(3,742)\end{array}$ & $\begin{array}{c}1,181 \\
(2,683)\end{array}$ & $\begin{array}{c}17,533 \\
(21,541)\end{array}$ & 458,727 \\
\hline Control & $\begin{array}{c}4,156 \\
(8,934)\end{array}$ & $\begin{array}{c}3,881 \\
(6,737)\end{array}$ & $\begin{array}{c}4,778 \\
(14,793)\end{array}$ & $\begin{array}{c}21,969 \\
(54,390)\end{array}$ & $\begin{array}{c}12,448 \\
(22,126)\end{array}$ & $\begin{array}{c}2,701 \\
(6,946)\end{array}$ & $\begin{array}{c}3,833 \\
(8,491)\end{array}$ & $\begin{array}{c}1,363 \\
(3,403)\end{array}$ & $\begin{array}{c}1,172 \\
(2,800)\end{array}$ & $\begin{array}{c}18,709 \\
(21,636)\end{array}$ & 449,220 \\
\hline Difference & $207.76^{ \pm * *}$ & $245.54^{* * *}$ & 131.35 & $-2,585.21$ & $466.61^{* \pm * *}$ & -48.34 & $163.72^{*}$ & $97.54^{* * *}$ & -9.5 & $1,176.24^{* * *}$ & \\
\hline
\end{tabular}

Table 3.2: Summary Statistics - Consumption and Debt 
Chapter 3. Follow the Money: The Effects of Liquidity Shocks on Consumption and Debt -

\begin{tabular}{|c|c|c|c|c|c|c|c|c|c|c|c|}
\hline & \multirow{2}{*}{\multicolumn{4}{|c|}{ Consumption Proxies }} & \multicolumn{6}{|c|}{ Debt } & \multirow{3}{*}{$\begin{array}{c}\text { Observations } \\
\text { (Individual- } \\
\text { month) }\end{array}$} \\
\hline & & & & & \multirow[b]{2}{*}{$\begin{array}{l}\text { Total } \\
\text { Debt } \\
\text { (E) }\end{array}$} & \multirow[b]{2}{*}{$\begin{array}{c}\text { Total } \\
\text { Overdue } \\
\text { (I) }\end{array}$} & \multirow[b]{2}{*}{$\begin{array}{c}\text { Total } \\
\text { Default } \\
\text { (J) }\end{array}$} & \multicolumn{3}{|c|}{ Selected Debt Modalities } & \\
\hline & $\begin{array}{c}\text { Total } \\
\text { Consumption } \\
\text { (A) }\end{array}$ & $\begin{array}{l}\text { Credit Card } \\
\text { Spending } \\
\text { (B) }\end{array}$ & $\begin{array}{l}\text { Vehicles } \\
\text { Down } \\
\text { Payments } \\
\text { (C) }\end{array}$ & $\begin{array}{l}\text { Real estate } \\
\text { Down } \\
\text { Payments } \\
\text { (D) }\end{array}$ & & & & $\begin{array}{l}\text { Credit Card } \\
\text { (F) }\end{array}$ & $\begin{array}{c}\text { Overdraft } \\
\text { (G) }\end{array}$ & $\begin{array}{l}\text { Payroll } \\
\text { (H) }\end{array}$ & \\
\hline \multicolumn{12}{|l|}{ Panel D. Group 4} \\
\hline \multicolumn{12}{|l|}{ Full Sample } \\
\hline Treatment & $\begin{array}{c}2,976 \\
(7,155) \\
4,300 \\
(10,119)\end{array}$ & $\begin{array}{c}2,784 \\
(4,916) \\
3,973 \\
(6,863)\end{array}$ & $\begin{array}{c}3,985 \\
(13,925) \\
5,052 \\
(14,775)\end{array}$ & $\begin{array}{c}25,269 \\
(67,335) \\
25,596 \\
(69,685)\end{array}$ & $\begin{array}{c}7,876 \\
(15,895) \\
13,237 \\
(23,430)\end{array}$ & $\begin{array}{c}2,585 \\
(6,619) \\
2,815 \\
(7,861)\end{array}$ & $\begin{array}{c}3,501 \\
(7,890) \\
4,068 \\
(13,176)\end{array}$ & $\begin{array}{c}1,168 \\
(3,080) \\
1,395 \\
(3,737)\end{array}$ & $\begin{array}{c}1,057 \\
(2,549) \\
1,221 \\
(3,024)\end{array}$ & $\begin{array}{c}13,059 \\
(16,996) \\
19,902 \\
(23,162)\end{array}$ & $1,932,518$ \\
\hline Difference & $1,324.24^{* * *}$ & $1,188.35^{* * *}$ & $1,066.77^{* * *}$ & 327.53 & $5,360.94^{* * *}$ & $230.16^{* * *}$ & $567.06^{* * *}$ & $226.55^{* * *}$ & $164.3^{* * * *}$ & $6,842.86^{* * *}$ & \\
\hline \multicolumn{12}{|c|}{ Matched Sample } \\
\hline Treatment & $\begin{array}{c}3,757 \\
(8,806)\end{array}$ & $\begin{array}{c}3,468 \\
(5,965)\end{array}$ & $\begin{array}{c}4,182 \\
(16,046)\end{array}$ & $\begin{array}{c}24,040 \\
(58,662)\end{array}$ & $\begin{array}{c}11,665 \\
(21,399)\end{array}$ & $\begin{array}{c}2,819 \\
(7,850)\end{array}$ & $\begin{array}{c}3,970 \\
(9,570)\end{array}$ & $\begin{array}{c}1,260 \\
(3,503)\end{array}$ & $\begin{array}{c}1,202 \\
(2,940)\end{array}$ & $\begin{array}{c}17,018 \\
(21,016)\end{array}$ & 461,145 \\
\hline Control & $\begin{array}{c}4,202 \\
(9,029)\end{array}$ & $\begin{array}{c}3,933 \\
(7,121)\end{array}$ & $\begin{array}{c}4,626 \\
(12,766)\end{array}$ & $\begin{array}{c}20,302 \\
(50,467)\end{array}$ & $\begin{array}{c}12,161 \\
(21,994)\end{array}$ & $\begin{array}{c}2,795 \\
(7,634)\end{array}$ & $\begin{array}{c}4,168 \\
(19,696)\end{array}$ & $\begin{array}{c}1,357 \\
(3,841)\end{array}$ & $\begin{array}{c}1,195 \\
(3,148)\end{array}$ & $\begin{array}{c}18,259 \\
(21,806)\end{array}$ & 450,561 \\
\hline Difference & $444.87^{* * *}$ & $465.22^{* * *}$ & 444.07 & $-3,737.99$ & $495.06^{* * *}$ & -24.16 & 197.26 & $96.43^{* * *}$ & -6.66 & $1,241.12^{* * *}$ & \\
\hline \multicolumn{12}{|l|}{ Panel E. Group 5} \\
\hline \multicolumn{12}{|l|}{ Full Sample } \\
\hline Treatment & $\begin{array}{c}2,970 \\
(6,823)\end{array}$ & $\begin{array}{c}2,782 \\
(4,925)\end{array}$ & $\begin{array}{c}3,714 \\
(11,734)\end{array}$ & $\begin{array}{c}23,849 \\
(56,858)\end{array}$ & $\begin{array}{c}7,885 \\
(16,148)\end{array}$ & $\begin{array}{c}2,611 \\
(6,921)\end{array}$ & $\begin{array}{c}3,482 \\
(8,355)\end{array}$ & $\begin{array}{c}1,175 \\
(2,987)\end{array}$ & $\begin{array}{c}1,049 \\
(2,401)\end{array}$ & $\begin{array}{c}13,026 \\
(17,158)\end{array}$ & 620,988 \\
\hline Control & $\begin{array}{c}4,312 \\
(10,341)\end{array}$ & $\begin{array}{c}3,994 \\
(7,247)\end{array}$ & $\begin{array}{c}4,991 \\
(13,874)\end{array}$ & $\begin{array}{c}24,741 \\
(68,995)\end{array}$ & $\begin{array}{c}13,494 \\
(23,828)\end{array}$ & $\begin{array}{c}2,820 \\
(7,610)\end{array}$ & $\begin{array}{c}4,096 \\
(9,392)\end{array}$ & $\begin{array}{l}1,418 \\
(3,770)\end{array}$ & $\begin{array}{c}1,258 \\
(3,119)\end{array}$ & $\begin{array}{c}20,231 \\
(23,487)\end{array}$ & 578,236 \\
\hline Difference & $1,341.57^{* * *}$ & $1,211.82^{* * *}$ & $1,276.99^{* * *}$ & 892.14 & $5,609.31^{* * *}$ & $208.62^{* * *}$ & $614.06^{* * *}$ & $242.99^{* * *}$ & $208.52^{* * * *}$ & $7,205.29^{* * *}$ & \\
\hline \multicolumn{12}{|c|}{ Matched Sample } \\
\hline Treatment & $\begin{array}{c}3,971 \\
(10,004)\end{array}$ & $\begin{array}{c}3,610 \\
(6,518)\end{array}$ & $\begin{array}{c}4,317 \\
(14,259)\end{array}$ & $\begin{array}{c}25,808 \\
(62,619)\end{array}$ & $\begin{array}{c}11,617 \\
(21,848)\end{array}$ & $\begin{array}{c}3,018 \\
(8,508)\end{array}$ & $\begin{array}{c}4,181 \\
(10,459)\end{array}$ & $\begin{array}{c}1,329 \\
(3,974)\end{array}$ & $\begin{array}{c}1,265 \\
(3,129)\end{array}$ & $\begin{array}{c}16,765 \\
(20,543)\end{array}$ & 147,114 \\
\hline Control & $\begin{array}{c}4,244 \\
(9,585)\end{array}$ & $\begin{array}{c}3,903 \\
(6,740)\end{array}$ & $\begin{array}{c}5,589 \\
(16,189)\end{array}$ & $\begin{array}{c}23,406 \\
(58,006)\end{array}$ & $\begin{array}{c}11,979 \\
(21,729)\end{array}$ & $\begin{array}{c}2,791 \\
(7,659)\end{array}$ & $\begin{array}{l}4,128 \\
(9,390)\end{array}$ & $\begin{array}{c}1,277 \\
(2,988)\end{array}$ & $\begin{array}{c}1,194 \\
(3,393)\end{array}$ & $\begin{array}{c}18,110 \\
(21,721)\end{array}$ & 145,534 \\
\hline Difference & $272.89 * * *$ & $292.69^{* * *}$ & 1272.03 & $-2,401.86$ & $361.92 * *$ & $-2,27.21^{\star \star *}$ & -53.44 & -52.31 & $-70.45^{\star}$ & $1,345.72^{* * *}$ & \\
\hline
\end{tabular}

\section{Summary Statistics - Consumption and Debt (Continued)}

Notes: This table reports the summary of consumption and debt statistics of our treatment and control sample both before and after propensity score matching (based on the nearest neighbor). The treatment sample consists of workers who qualify for the Withdrawal Program of the FGTS in 2017 (workers who had an inactive account of the FGTS until December, 31 2015) and the control sample consists of all workers who did not qualified for the program (individuals with FGTS accounts created after December 31, 2015 or with accounts cleared by withdrawals made before the program announcement). We exclude individuals with no information in the SCR database in our period sample (2016:05-2017:12). It shows the comparison between treatment and control clusters in consumption categories and debt modalities. We also include the total overdue debt and total default debt. See the main text of section 3 for more details on the program timeline for each group and definitions on debt modalities. All the amounts are in the local currency (BRL\$), and US $\$ 1=$ BRL $\$ 3.2591$ as of December 2016. 

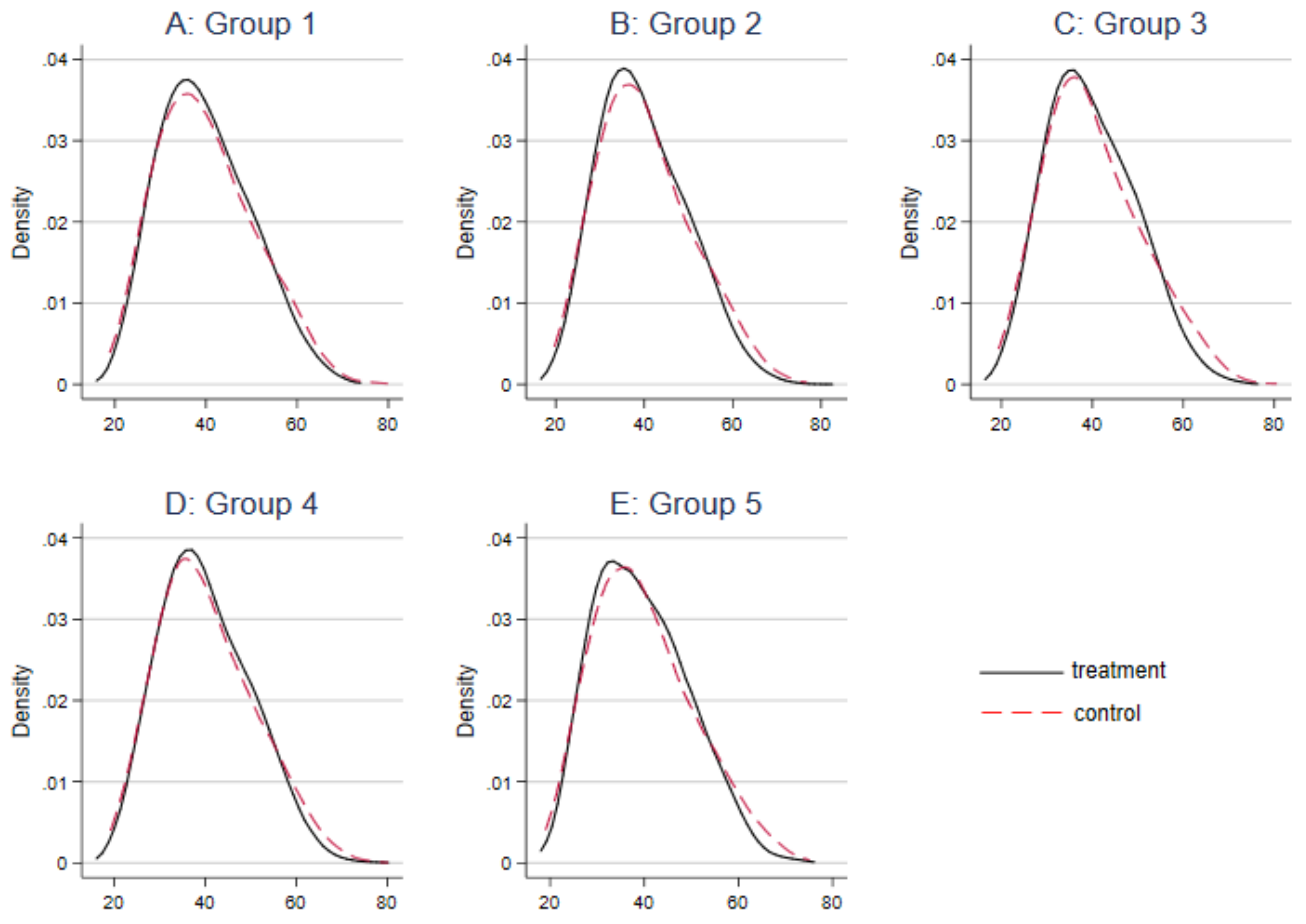

Figure 3.2: Kernel Densities of the Matched Sample - Age

Notes: This figure shows the treatment group and the control group comparison of distributions of age during the period 2016:01-2016:04 after the propensity score matching for the five groups of treated workers. 
A: Group 1

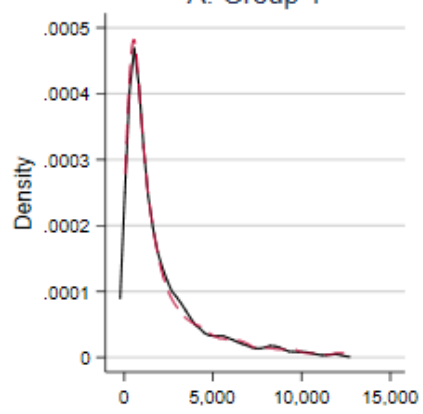

D: Group 4

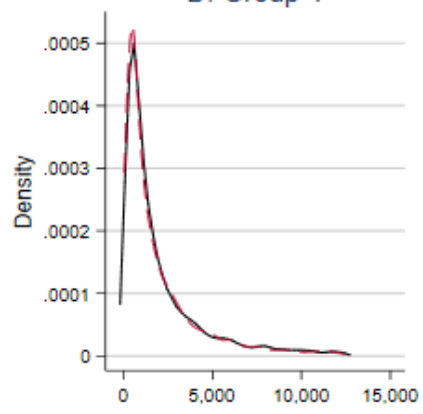

B: Group 2

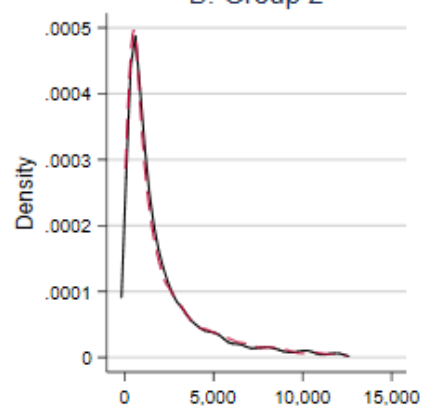

E: Group 5

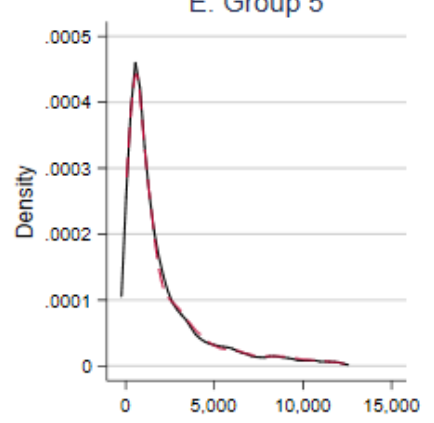

C: Group 3
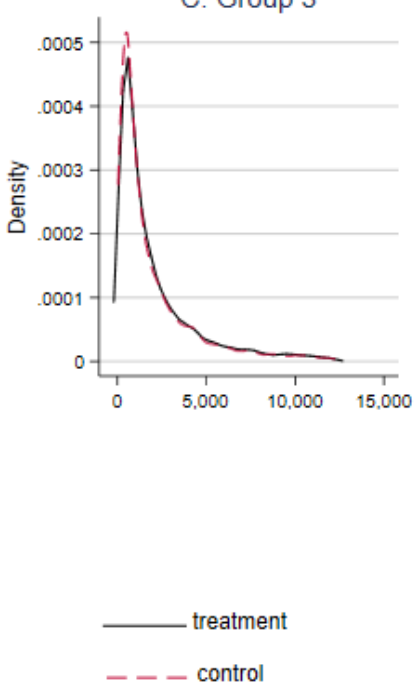

Figure 3.3: Kernel Densities of the Matched Sample - Monthly Income in 2016

Notes: This figure shows the treatment group and the control group comparison of distributions of monthly income in 2016 during the period 2016:01-2016:04 after the propensity score matching for the five groups of treated workers. 


\section{A: Group 1}

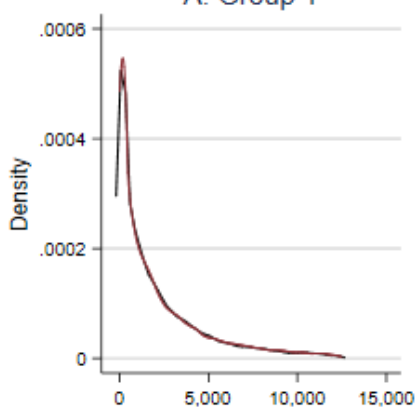

D: Group 4

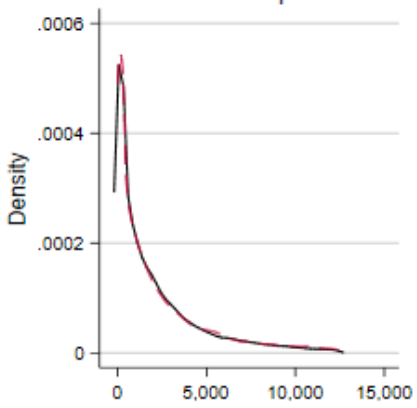

B: Group 2

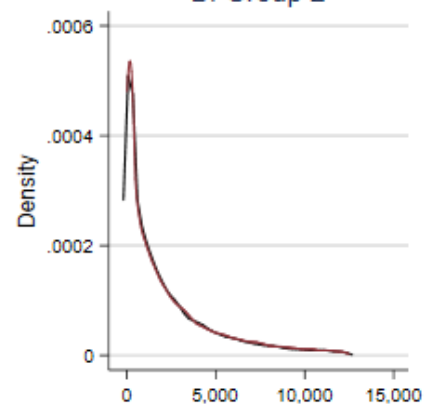

E: Group 5

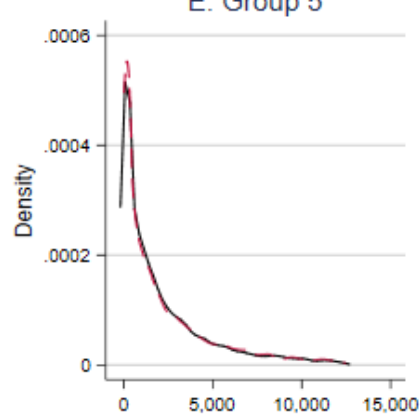

C: Group 3
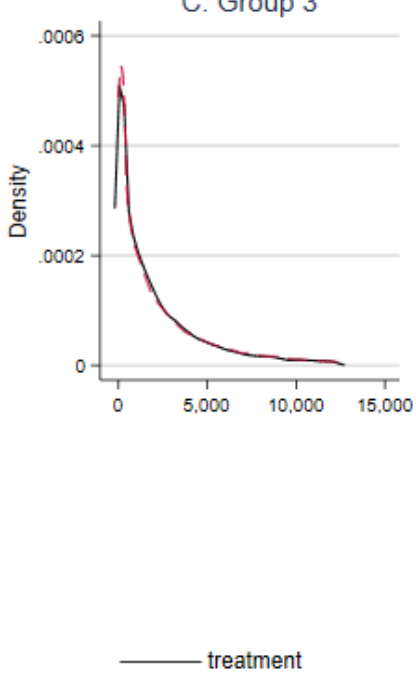

Figure 3.4: Kernel Densities of the Matched Sample - Monthly Credit Card Spending in 2016

Notes: This figure shows the treatment group and the control group comparison of distributions of monthly credit card spending in 2016 during the period 2016:01-2016:04 after the propensity score matching for the five groups of treated workers. 
Chapter 3. Follow the Money: The Effects of Liquidity Shocks on Consumption and Debt -

Matched Sample

(A): Group 1

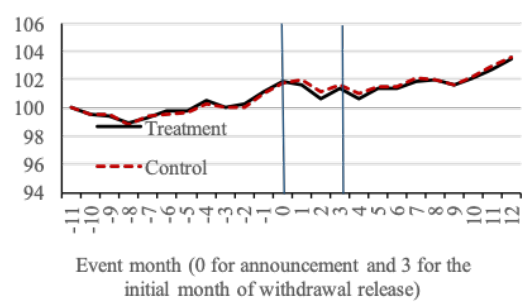

(B): Group 2

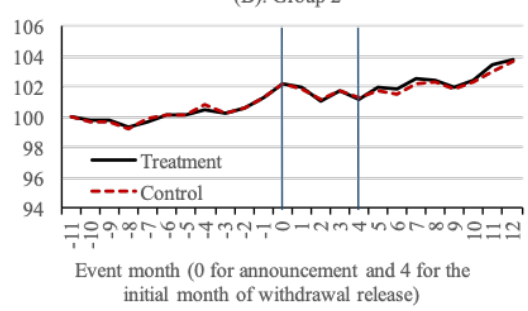

(C): Group 3

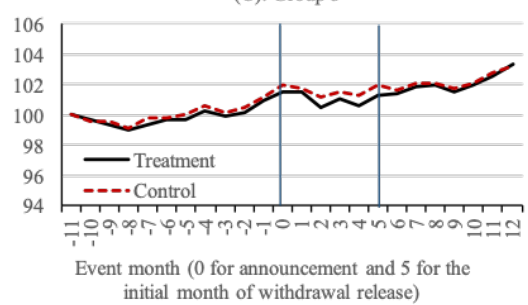

(D): Group 4

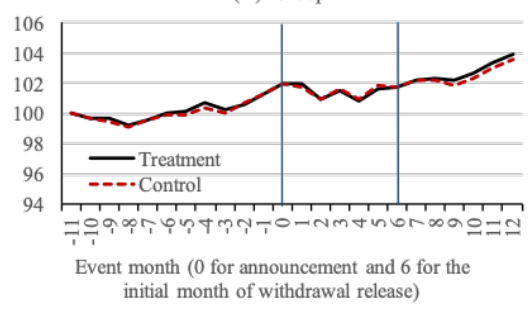

(E): Group 5

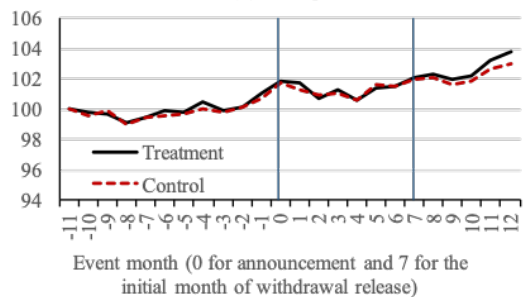

Full Sample

(A): Group 1

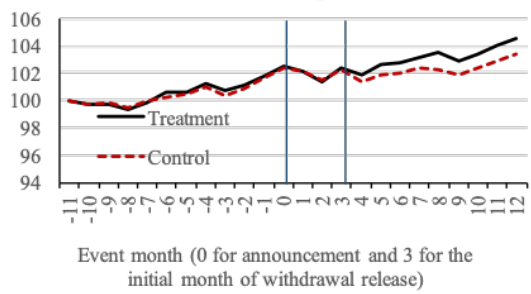

(B): Group 2

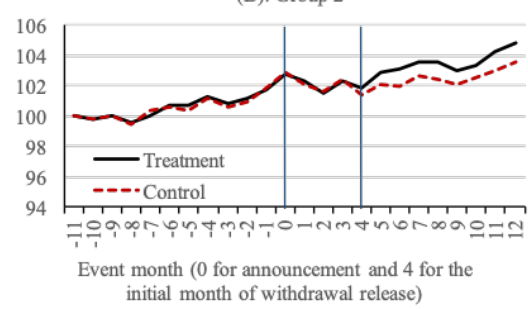

(C): Group 3

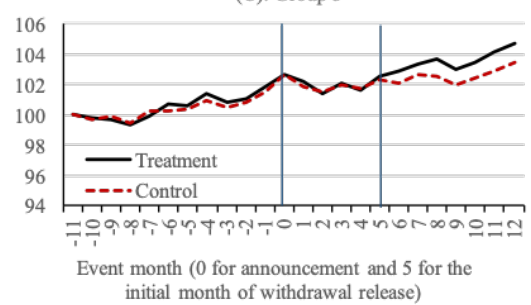

(D): Group 4

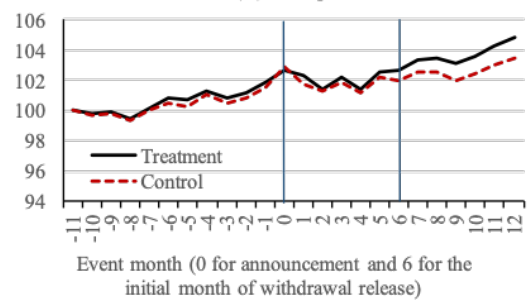

(E): Group 5

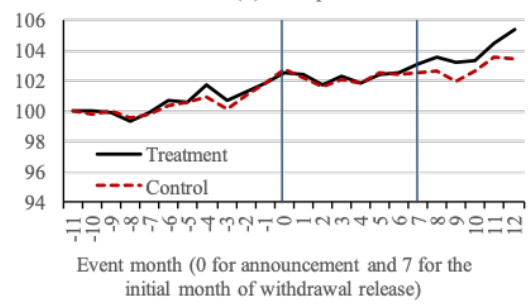

Figure 3.5: Unconditional Mean of Consumption

Notes: This figure depicts the comparison of the unconditional mean of consumption between the treatment and control clusters for all five groups of treated workers in the matched sample and in the full sample in the period from 2016:01-2017:12. In the full sample workers are weighted by their respective propensity scores. Numbers are normalized relative to period $-11(2016: 01=100)$. For more details on the program timeline for each group and definitions of consumption and debt modalities as well as details on the propensty score matching applied to treated and untreated workers see the main text of section 3 and appendix A, respectively. 
Chapter 3. Follow the Money: The Effects of Liquidity Shocks on Consumption and Debt -

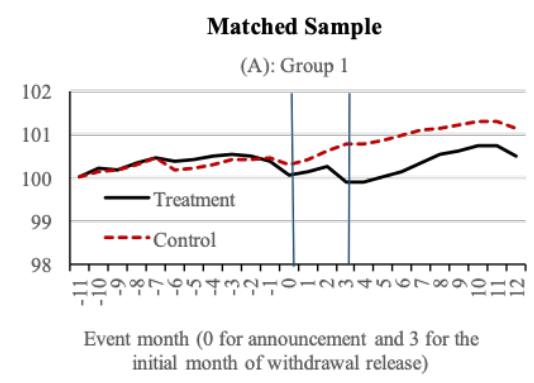

(B): Group 2

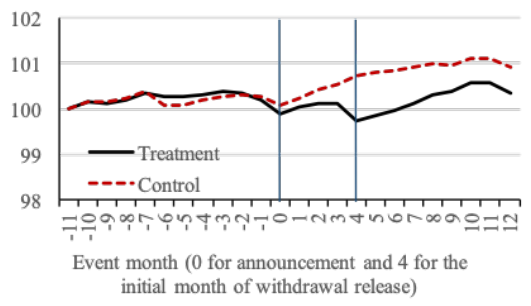

(C): Group 3

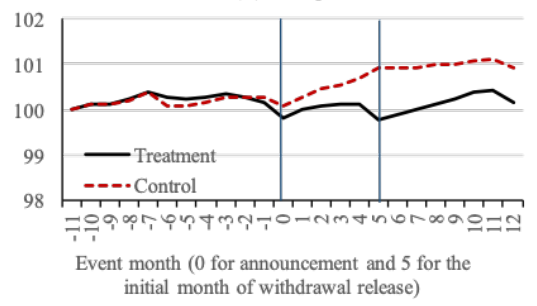

(D): Group 4

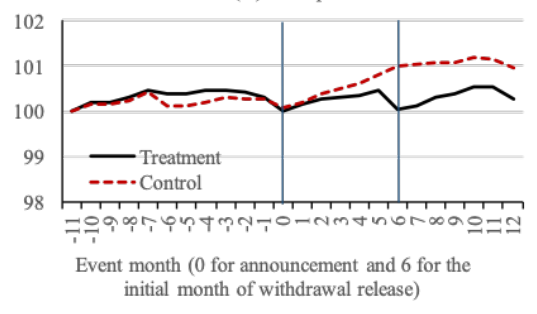

(E): Group 5

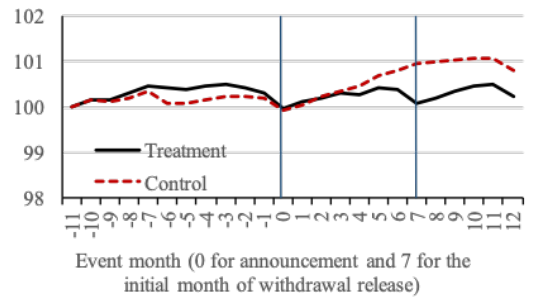

Full Sample

(A): Group 1

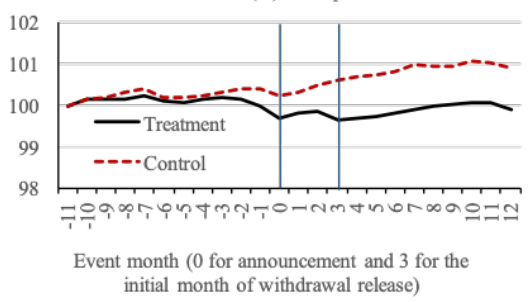

(B): Group 2

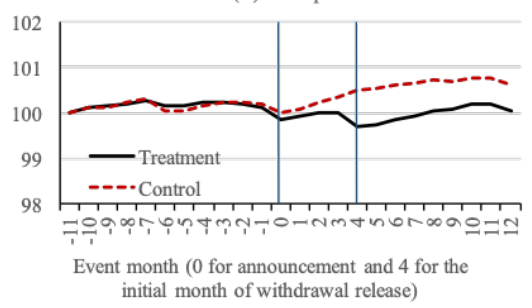

(C): Group 3

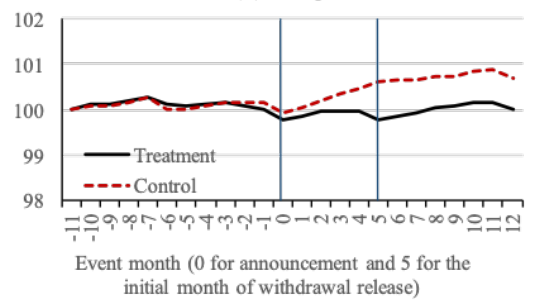

(D): Group 4

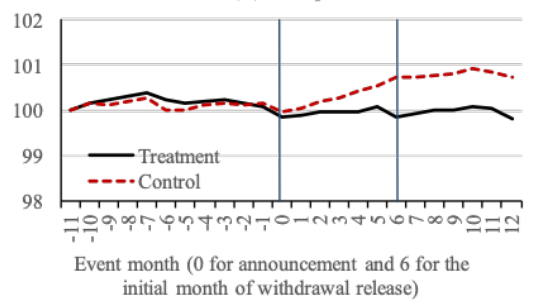

(E): Group 5

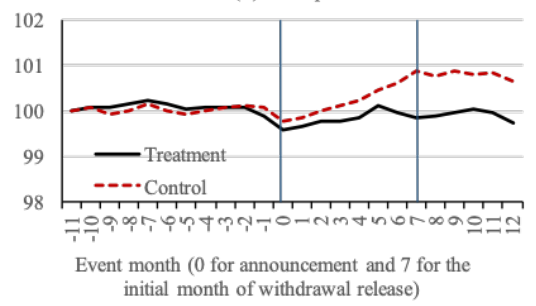

Figure 3.6: Unconditional Mean of Total Debt

Notes: This figure depicts the comparison of the unconditional mean of total debt between the treatment and control clusters for all five groups of treated workers in the matched sample and in the full sample in the period from 2016:01-2017:12. In the full sample workers are weighted by their respective propensity scores. Numbers are normalized relative to period $-11(2016: 01=100)$. For more details on the program timeline for each group and definitions of consumption and debt modalities as well as details on the propensty score matching applied to treated and untreated workers see the main text of section 3 and appendix A, respectively. 
Chapter 3. Follow the Money: The Effects of Liquidity Shocks on Consumption and Debt -

\begin{tabular}{|c|c|c|c|c|c|}
\hline & $\begin{array}{c}\text { Group } 1 \\
\text { (A) }\end{array}$ & $\begin{array}{c}\text { Group } 2 \\
\text { (B) }\end{array}$ & $\begin{array}{c}\text { Group } 3 \\
\text { (C) }\end{array}$ & $\begin{array}{c}\text { Group } 4 \\
\text { (D) }\end{array}$ & $\begin{array}{c}\text { Group } 5 \\
\text { (E) }\end{array}$ \\
\hline \multicolumn{6}{|l|}{ Panel A } \\
\hline$\$ W^{g} \times 1_{\text {pre }}$ & $\begin{array}{l}-0.001 \\
(-0.08)\end{array}$ & $\begin{array}{l}0.001 \\
(0.11)\end{array}$ & $\begin{array}{l}0.011 \\
(0.88)\end{array}$ & $\begin{array}{l}-0.009 \\
(-0.74)\end{array}$ & $\begin{array}{l}0.003 \\
(0.12)\end{array}$ \\
\hline$\$ W^{g} \times 1_{\text {post }}$ & $\begin{array}{c}0.038^{* *} \\
(2.11)\end{array}$ & $\begin{array}{c}0.058^{* * *} \\
(3.53)\end{array}$ & $\begin{array}{c}0.056^{* * * *} \\
(3.73)\end{array}$ & $\begin{array}{c}0.029 * * \\
(2.14)\end{array}$ & $\begin{array}{r}0.045^{*} \\
(1.83)\end{array}$ \\
\hline Constant & $\begin{array}{c}2,815.811^{* * * *} \\
(84.49)\end{array}$ & $\begin{array}{c}2,912.667 * * * \\
(118.77)\end{array}$ & $\begin{array}{c}2,958.918^{* * *} \\
(91.71)\end{array}$ & $\begin{array}{c}2,823.029 * * * \\
(102.22)\end{array}$ & $\begin{array}{c}2,878.430^{* * *} \\
(76.21)\end{array}$ \\
\hline$R^{2}$ & 0.41 & 0.41 & 0.42 & 0.43 & 0.46 \\
\hline \multicolumn{6}{|l|}{ Panel B } \\
\hline$\$ W^{g} \times 1_{\text {pre }}$ & $\begin{array}{l}-0.001 \\
(-0.08)\end{array}$ & $\begin{array}{l}0.001 \\
(0.11)\end{array}$ & $\begin{array}{l}0.011 \\
(0.88)\end{array}$ & $\begin{array}{l}-0.009 \\
(-0.74)\end{array}$ & $\begin{array}{l}0.003 \\
(0.12)\end{array}$ \\
\hline$\$ W^{g} \times 1_{\text {announce }}^{g}$ & $\begin{array}{l}0.024 \\
(1.09)\end{array}$ & $\begin{array}{c}0.030 * * \\
(2.10)\end{array}$ & $\begin{array}{c}0.031 * * \\
(2.11)\end{array}$ & $\begin{array}{l}0.002 \\
(0.19)\end{array}$ & $\begin{array}{l}0.004 \\
(0.19)\end{array}$ \\
\hline$\$ W^{g} \times 1_{\text {withdraw }}^{g}$ & $\begin{array}{c}0.046^{* *} \\
(2.50)\end{array}$ & $\begin{array}{c}0.076^{* * *} \\
(3.82)\end{array}$ & $\begin{array}{c}0.077^{* * *} \\
(4.51)\end{array}$ & $\begin{array}{c}0.055^{* * *} \\
(3.43)\end{array}$ & $\begin{array}{c}0.092 * * * \\
(3.25)\end{array}$ \\
\hline Constant & $\begin{array}{c}2,815.817 * * * \\
(84.49)\end{array}$ & $\begin{array}{c}2,912.677 * * * \\
\quad(118.77)\end{array}$ & $\begin{array}{c}2,958.927 * * * \\
\quad(91.71)\end{array}$ & $\begin{array}{c}2,823.041^{* * *} \\
\quad(102.22)\end{array}$ & $\begin{array}{c}2,878.459 * * * \\
(76.21)\end{array}$ \\
\hline$R^{2}$ & 0.41 & 0.41 & 0.42 & 0.43 & 0.46 \\
\hline Fixed effects & \multicolumn{5}{|c|}{ Individual, year-month } \\
\hline Observations & 600,943 & $1,017,483$ & $1,067,932$ & $1,137,881$ & 385,37 \\
\hline
\end{tabular}

Table 3.3: The Average Consumption Response to the FGTS Program

Notes: This table shows the average consumption response (equations (1) and (2)) in the period from from 2016:05 to 2017:12 using the matched sample. Panel A presents the estimation results of equation (1), and panel B shows the estimation results of equation (2). $\$ W^{g}$ is the amount withdrawn by the treatment group $g$, and is equal to 0 for the control group. $1_{\text {pre }}$ is a binary variable equal to 1 for the month before the announcement (i.e., 2016:05-2016:11). $1_{\text {post }}$ is a binary variable equal to 1 for the months after the announcement of the Withdrawals Program (i.e., $\geq 2016: 12$ ). $1_{\text {announce }}^{g}$ equal to 1 for $g=\{1,2,3,4,5\}$ in the following month ranges: 2016:12 to 2017:02, 2016:12 to 2017:03, 2016:12 to 2017:04, 2016:12 to 2017:05, 2016:12 to 2017:06, respectively. $1_{\text {withdraw }}^{g}$ equal to 1 for $g=\{1,2,3,4,5\}$ in the following month ranges: $2016: 03$ to $2017: 08,2016: 04$ to $2017: 09,2016: 05$ to $2017: 10,2016: 06$ to $2017: 11,2016: 07$ to $2017: 12$, respectively. Individual and year-month fixed effects are included, and standard errors are clustered at the individual level. T-statistics are reported in parentheses under the coefficient estimates. *** Significant at the 1 percent level. ** Significant at the 5 percent level. * Significant at the 10 percent level. 
Chapter 3. Follow the Money: The Effects of Liquidity Shocks on Consumption and Debt -

\begin{tabular}{|c|c|c|c|c|c|}
\hline & $\begin{array}{l}\text { Group } 1 \\
\text { (A) }\end{array}$ & $\begin{array}{c}\text { Group } 2 \\
\text { (B) }\end{array}$ & $\begin{array}{c}\text { Group } 3 \\
\text { (C) }\end{array}$ & $\begin{array}{l}\text { Group } 4 \\
\text { (D) }\end{array}$ & $\begin{array}{c}\text { Group } 5 \\
\text { (E) }\end{array}$ \\
\hline \multicolumn{6}{|l|}{ Panel A } \\
\hline$\$ W^{g} \times 1_{\text {pre }}$ & $\begin{array}{l}0.011 \\
(0.75)\end{array}$ & $\begin{array}{l}0.004 \\
(0.33)\end{array}$ & $\begin{array}{l}0.003 \\
(0.27)\end{array}$ & $\begin{array}{l}-0.022 \\
(-1.53)\end{array}$ & $\begin{array}{l}-0.028 \\
(-1.38)\end{array}$ \\
\hline$\$ W^{g} \times 1_{\text {post }}$ & $\begin{array}{c}-0.062^{* *} \\
(-2.34)\end{array}$ & $\begin{array}{c}-0.046^{* *} \\
(-2.09)\end{array}$ & $\begin{array}{l}-0.037 \\
(-1.46)\end{array}$ & $\begin{array}{c}-0.073 * * * \\
(-2.77)\end{array}$ & $\begin{array}{l}-0.13 * * * \\
(-3.04)\end{array}$ \\
\hline Constant & $\begin{array}{c}9,759.351 * * * \\
(216.75)\end{array}$ & $\begin{array}{c}9,890.181^{* * *}(2 ; \\
.12)\end{array}$ & $\begin{array}{c}9,892.497 * * * \\
(271.16)\end{array}$ & $\begin{array}{c}9,635.258^{* * *} \\
(253.82)\end{array}$ & $\begin{array}{c}9,543.264 * * * \\
(126.54)\end{array}$ \\
\hline$R^{2}$ & 0.90 & 0.90 & 0.90 & 0.89 & 0.86 \\
\hline \multicolumn{6}{|l|}{ Panel B } \\
\hline$\$ W^{g} \times 1_{\text {pre }}$ & $\begin{array}{l}0.011 \\
(0.75)\end{array}$ & $\begin{array}{l}0.004 \\
(0.33)\end{array}$ & $\begin{array}{l}0.003 \\
(0.27)\end{array}$ & $\begin{array}{l}-0.022 \\
(-1.53)\end{array}$ & $\begin{array}{l}-0.028 \\
(-1.38)\end{array}$ \\
\hline$\$ W^{g} \times 1_{\text {announce }}^{g}$ & $\begin{array}{l}0.003 \\
(0.14)\end{array}$ & $\begin{array}{l}0.006 \\
(0.29)\end{array}$ & $\begin{array}{l}0.000 \\
(-0.01)\end{array}$ & $\begin{array}{l}-0.036 \\
(-1.48)\end{array}$ & $\begin{array}{c}-0.084 * * \\
(-2.14)\end{array}$ \\
\hline$\$ W^{g} \times 1_{\text {withdraw }}^{g}$ & $\begin{array}{c}-0.094^{* * *} \\
(-3.24)\end{array}$ & $\begin{array}{c}-0.081^{* * *} \\
(-3.28)\end{array}$ & $\begin{array}{c}-0.068^{* *} \\
(-2.39)\end{array}$ & $\begin{array}{c}-0.110 * * * \\
(-3.67)\end{array}$ & $\begin{array}{c}-0.183^{* * *} \\
(-3.67)\end{array}$ \\
\hline Constant & $\begin{array}{l}9,543.264 * * * \\
\quad(126.54)\end{array}$ & $\begin{array}{c}9,890.163^{* * * *} \\
(274.12)\end{array}$ & $\begin{array}{l}9,892.484 * * * \\
\quad(271.16)\end{array}$ & $\begin{array}{l}9,635.241^{* * *} \\
\quad(253.82)\end{array}$ & $\begin{array}{c}9,543.231^{* * *} \\
\quad(126.54)\end{array}$ \\
\hline$R^{2}$ & 0.90 & 0.90 & 0.90 & 0.89 & 0.86 \\
\hline Fixed effects & \multicolumn{5}{|c|}{ Individual, year-month } \\
\hline Observations & 600,943 & $1,017,483$ & $1,067,932$ & $1,137,881$ & 385,37 \\
\hline
\end{tabular}

Table 3.4: The Average Total Debt Response to the FGTS Program

Notes: This table shows the average total debt response (equations (1) and (2)) in the period from from 2016:05 to 2017:12 using the matched sample. Panel A presents the estimation results of equation (1), and panel B shows the estimation results of equation (2). $\$ W^{g}$ is the amount withdrawn by the treatment group $g$, and is equal to 0 for the control group. $1_{\text {pre }}$ is a binary variable equal to 1 for the month before the announcement (i.e., 2016:05-2016:11). $1_{\text {post }}$ is a binary variable equal to 1 for the months after the announcement of the Withdrawals Program (i.e., $\geq 2016: 12$ ). $1_{\text {announce }}^{g}$ equal to 1 for $g=\{1,2,3,4,5\}$ in the following month ranges: 2016:12 to 2017:02, 2016:12 to 2017:03, 2016:12 to 2017:04, 2016:12 to 2017:05, 2016:12 to 2017:06, respectively. $1_{\text {withdraw }}^{g}$ equal to 1 for $g=\{1,2,3,4,5\}$ in the following month ranges: 2016:03 to 2017:08, 2016:04 to 2017:09, 2016:05 to 2017:10, 2016:06 to 2017:11, 2016:07 to 2017:12, respectively. Individual and year-month fixed effects are included, and standard errors are clustered at the individual level. T-statistics are reported in parentheses under the coefficient estimates. ${ }^{* * *}$ Significant at the 1 percent level. ${ }^{* *}$ Significant at the 5 percent level. ${ }^{*}$ Significant at the 10 percent level. 
Chapter 3. Follow the Money: The Effects of Liquidity Shocks on Consumption and Debt -

\begin{tabular}{|c|c|c|c|c|c|}
\hline & $\begin{array}{l}\text { Group } 1 \\
\text { (A) }\end{array}$ & $\begin{array}{l}\text { Group } 2 \\
\text { (B) }\end{array}$ & $\begin{array}{l}\text { Group } 3 \\
\text { (C) }\end{array}$ & $\begin{array}{l}\text { Group } 4 \\
\text { (D) }\end{array}$ & $\begin{array}{c}\text { Group } 5 \\
\text { (E) }\end{array}$ \\
\hline$\beta_{0}^{g}$ & $\begin{array}{l}0.038 \\
(1.31)\end{array}$ & $\begin{array}{c}0.025^{*} \\
(1.75)\end{array}$ & $\begin{array}{c}0.039 * * \\
(2.09)\end{array}$ & $\begin{array}{l}-0.007 \\
(-0.40)\end{array}$ & $\begin{array}{l}0.001 \\
(0.04)\end{array}$ \\
\hline$\beta_{1}^{g}$ & $\begin{array}{l}0.020 \\
(0.77)\end{array}$ & $\begin{array}{c}0.037^{* *} \\
(2.36)\end{array}$ & $\begin{array}{c}0.046^{* * *} \\
(2.66)\end{array}$ & $\begin{array}{l}0.019 \\
(1.32)\end{array}$ & $\begin{array}{l}0.024 \\
(0.92)\end{array}$ \\
\hline$\beta_{2}^{g}$ & $\begin{array}{l}0.014 \\
(0.44)\end{array}$ & $\begin{array}{l}0.026 \\
(1.51)\end{array}$ & $\begin{array}{l}0.021 \\
(1.18)\end{array}$ & $\begin{array}{l}-0.008 \\
(-0.63)\end{array}$ & $\begin{array}{l}-0.021 \\
(-0.80)\end{array}$ \\
\hline$\beta_{3}^{g}$ & $\begin{array}{l}0.027 \\
(0.98)\end{array}$ & $\begin{array}{c}0.031^{*} \\
(1.75)\end{array}$ & $\begin{array}{c}0.031 * \\
(1.87)\end{array}$ & $\begin{array}{l}0.001 \\
(0.04)\end{array}$ & $\begin{array}{l}-0.003 \\
(-0.10)\end{array}$ \\
\hline$\beta_{4}^{g}$ & $\begin{array}{l}0.010 \\
(0.53)\end{array}$ & $\begin{array}{c}0.041 * * \\
(2.19)\end{array}$ & $\begin{array}{l}0.018 \\
(1.13)\end{array}$ & $\begin{array}{l}-0.005 \\
(-0.42)\end{array}$ & $\begin{array}{l}-0.008 \\
(-0.31)\end{array}$ \\
\hline$\beta_{5}^{g}$ & $\begin{array}{c}0.043 * * \\
(2.04)\end{array}$ & $\begin{array}{c}0.065 * * * \\
(3.66)\end{array}$ & $\begin{array}{c}0.083 * * \\
(2.41)\end{array}$ & $\begin{array}{l}0.015 \\
(0.89)\end{array}$ & $\begin{array}{l}-0.002 \\
(-0.06)\end{array}$ \\
\hline$\beta_{6}^{g}$ & $\begin{array}{c}0.055 * * \\
(2.40)\end{array}$ & $\begin{array}{c}0.073 * * * \\
(3.59)\end{array}$ & $\begin{array}{c}0.070 * * * \\
(3.72)\end{array}$ & $\begin{array}{c}0.068 * * * \\
(2.82)\end{array}$ & $\begin{array}{l}0.040 \\
(1.09)\end{array}$ \\
\hline$\beta_{7}^{g}$ & $\begin{array}{c}0.056 * * \\
(2.05)\end{array}$ & $\begin{array}{c}0.159 * * \\
(2.12)\end{array}$ & $\begin{array}{c}0.078 * * * \\
(3.66)\end{array}$ & $\begin{array}{c}0.070 * * * \\
(3.56)\end{array}$ & $\begin{array}{c}0.079 * * \\
(2.22)\end{array}$ \\
\hline$\beta_{8}^{g}$ & $\begin{array}{c}0.084 * * * \\
(3.14)\end{array}$ & $\begin{array}{c}0.048 * * * \\
(2.82)\end{array}$ & $\begin{array}{c}0.096 * * * \\
(3.74)\end{array}$ & $\begin{array}{c}0.040 * * \\
(2.29)\end{array}$ & $\begin{array}{c}0.125 * * * \\
(2.89)\end{array}$ \\
\hline$\beta_{9}^{g}$ & & $\begin{array}{c}0.072 * * * \\
(3.76)\end{array}$ & $\begin{array}{c}0.055 * * * \\
(2.95)\end{array}$ & $\begin{array}{c}0.052 * * * \\
(2.75)\end{array}$ & $\begin{array}{c}0.067 * * \\
(2.33)\end{array}$ \\
\hline$\beta_{10}^{g}$ & & & $\begin{array}{c}0.082 * * * \\
(3.49)\end{array}$ & $\begin{array}{c}0.049 * * \\
(2.52)\end{array}$ & $\begin{array}{c}0.065 * * \\
(2.24)\end{array}$ \\
\hline$\beta_{11}^{g}$ & & & & $\begin{array}{c}0.051 * * * \\
(3.19)\end{array}$ & $\begin{array}{c}0.096 * * * \\
(2.91)\end{array}$ \\
\hline$\beta_{12}^{g}$ & & & & & $\begin{array}{c}0.121 * * * \\
(3.60)\end{array}$ \\
\hline$c_{p r e}^{g}$ & $\begin{array}{l}-0.008 \\
(-0.08)\end{array}$ & $\begin{array}{l}0.007 \\
(0.11)\end{array}$ & $\begin{array}{l}0.067 \\
(0.88)\end{array}$ & $\begin{array}{l}-0.052 \\
(-0.74)\end{array}$ & $\begin{array}{l}0.018 \\
(0.12)\end{array}$ \\
\hline$c_{\text {announce }}^{g}$ & $\begin{array}{l}0.071 \\
(1.08)\end{array}$ & $\begin{array}{c}0.119^{* *} \\
(2.10)\end{array}$ & $\begin{array}{c}0.155^{* *} \\
(2.11)\end{array}$ & $\begin{array}{l}0.014 \\
(0.19)\end{array}$ & $\begin{array}{l}0.031 \\
(0.19)\end{array}$ \\
\hline$c_{\text {withdrawal }}^{g}$ & $\begin{array}{c}0.275^{* *} \\
(2.50)\end{array}$ & $\begin{array}{c}0.459 * * * \\
(3.82)\end{array}$ & $\begin{array}{c}0.464 * * * \\
(4.51)\end{array}$ & $\begin{array}{c}0.329 * * * \\
(3.43)\end{array}$ & $\begin{array}{c}0.553 * * * \\
(3.25)\end{array}$ \\
\hline
\end{tabular}

Fixed-effects

Individual, year-month

$\begin{array}{lllll}\text { Observations } & 600,943 \quad 1,017,483 & 1,067,932 & 1,137,881 & 385,370\end{array}$

Table 3.5: The Consumption Response Dynamics

Notes: This table shows the consumption response dynamics (equation (3)) in the period from from 2016:05 to 2017:12 using the matched sample. $\beta_{s}^{g}$ measures the coefficient estimates of variable $\$ W_{i}^{g} \times 1_{\text {month } s}$ and represents the average monthly response in month $s=\{-1,0, \ldots, 12\}$, where $s=-6$ stands for 2016:06,..., $s=0$ is the announcement month $2016: 12, \ldots$, and $s=12$ is the last month in the withdrawal period of group $g=5,2017: 12$. We define $c_{p r e}^{g} \equiv \sum_{t=-6}^{-1} \beta_{t}^{g}$ as the cumulative consumption response within the pre-treatment window for group $g$, where $g=\{1,2,3,4,5\}$. We decompose post-treatment period into announcement and withdrawal periods. $c_{\text {announce }}^{g} \equiv \sum_{t=0}^{\tau^{g}-1} \beta_{t}^{g}$, where $\tau^{g}=\{3,4,5,6,7\}$ is the initial month of withdrawals release for groups $g=\{1,2,3,4,5\}$, respectively, is the cumulative consumption response of the program in group $g$ within the announcement window. $c_{\text {withdraw }}^{g} \equiv \sum_{t=\tau^{g}}^{S^{g}} \beta_{t}^{g}$, in turn, is the cumulative consumption response of the program in group $g$ within the withdrawal period, where $S^{g}=\{7,8,9,10,11,12\}$ for group $g=\{1,2,3,4,5\}$, respectively. Withdrawal windows for each group are in bold. Individual and year-month fixed effects are included, and standard errors are clustered at the individual level. T-statistics are reported in parentheses under the coefficient estimates. ${ }^{* * *}$ Significant at the 1 percent level. ${ }^{* *}$ Significant at the 5 percent level. * Significant at the 10 percent level. 
Chapter 3. Follow the Money: The Effects of Liquidity Shocks on Consumption and Debt -

\begin{tabular}{|c|c|c|c|c|c|}
\hline & $\begin{array}{l}\text { Group } 1 \\
\text { (A) }\end{array}$ & $\begin{array}{l}\text { Group } 2 \\
\text { (B) }\end{array}$ & $\begin{array}{l}\text { Group } 3 \\
\text { (C) }\end{array}$ & $\begin{array}{l}\text { Group } 4 \\
\text { (D) }\end{array}$ & $\begin{array}{c}\text { Group } 5 \\
\text { (E) }\end{array}$ \\
\hline$\beta_{0}^{g}$ & $\begin{array}{l}-0.013 \\
(-0.58)\end{array}$ & $\begin{array}{l}-0.007 \\
(-0.31)\end{array}$ & $\begin{array}{l}-0.009 \\
(-0.43)\end{array}$ & $\begin{array}{c}-0.043^{*} \\
(-1.89)\end{array}$ & $\begin{array}{c}-0.082^{* *} \\
(-2.31)\end{array}$ \\
\hline$\beta_{1}^{g}$ & $\begin{array}{l}0.006 \\
(0.25)\end{array}$ & $\begin{array}{l}0.006 \\
(0.25)\end{array}$ & $\begin{array}{l}0.001 \\
(0.05)\end{array}$ & $\begin{array}{l}-0.033 \\
(-1.39)\end{array}$ & $\begin{array}{c}-0.076^{* *} \\
(-2.00)\end{array}$ \\
\hline$\beta_{2}^{g}$ & $\begin{array}{l}0.016 \\
(0.61)\end{array}$ & $\begin{array}{l}0.017 \\
(0.80)\end{array}$ & $\begin{array}{l}0.000 \\
(0.01)\end{array}$ & $\begin{array}{l}-0.029 \\
(-1.15)\end{array}$ & $\begin{array}{c}-0.069^{*} \\
(-1.71)\end{array}$ \\
\hline$\beta_{3}^{g}$ & $\begin{array}{c}-0.076 * * * \\
(-2.76)\end{array}$ & $\begin{array}{l}0.008 \\
(0.37)\end{array}$ & $\begin{array}{l}0.001 \\
(0.05)\end{array}$ & $\begin{array}{l}-0.039 \\
(-1.47)\end{array}$ & $\begin{array}{c}-0.087^{* *} \\
(-2.18)\end{array}$ \\
\hline$\beta_{4}^{g}$ & $\begin{array}{c}-0.096 * * * \\
(-3.34)\end{array}$ & $\begin{array}{c}-0.076 * * * \\
(-3.29)\end{array}$ & $\begin{array}{l}0.005 \\
(0.19)\end{array}$ & $\begin{array}{l}-0.041 \\
(-1.55)\end{array}$ & $\begin{array}{c}-0.087 * * \\
(-2.07)\end{array}$ \\
\hline$\beta_{5}^{g}$ & $\begin{array}{c}-0.102 * * * \\
(-3.47)\end{array}$ & $\begin{array}{c}-0.096 * * * \\
(-3.96)\end{array}$ & $\begin{array}{c}-0.079 * * * \\
(-2.89)\end{array}$ & $\begin{array}{l}-0.034 \\
(-1.23)\end{array}$ & $\begin{array}{c}-0.096^{* *} \\
(-2.03)\end{array}$ \\
\hline$\beta_{6}^{g}$ & $\begin{array}{c}-0.098 * * * \\
(-3.21)\end{array}$ & $\begin{array}{c}-0.097 * * * \\
(-3.82)\end{array}$ & $\begin{array}{c}-0.074 * * * \\
(-2.56)\end{array}$ & $\begin{array}{c}-0.117 * * * \\
(-4.22)\end{array}$ & $\begin{array}{c}-0.093 * * \\
(-1.97)\end{array}$ \\
\hline$\beta_{7}^{g}$ & $\begin{array}{c}-0.110 * * * \\
(-3.51)\end{array}$ & $\begin{array}{c}-0.086 * * * \\
(-3.29)\end{array}$ & $\begin{array}{c}-0.060 * * \\
(-2.07)\end{array}$ & $\begin{array}{c}-0.120 * * * \\
(-4.16)\end{array}$ & $\begin{array}{c}-0.198 * * * \\
(-4.15)\end{array}$ \\
\hline$\beta_{8}^{g}$ & $\begin{array}{c}-0.085 * * \\
(-2.54)\end{array}$ & $\begin{array}{c}-0.070 * * \\
(-2.53)\end{array}$ & $\begin{array}{c}-0.065 * * \\
(-2.22)\end{array}$ & $\begin{array}{c}-0.121 * * * \\
(-4.01)\end{array}$ & $\begin{array}{c}-0.196 * * * \\
(-4.08)\end{array}$ \\
\hline$\beta_{9}^{g}$ & & $\begin{array}{c}-0.060 * * \\
(-2.06)\end{array}$ & $\begin{array}{c}-0.063 * * \\
(-2.03)\end{array}$ & $\begin{array}{c}-0.108 * * * \\
(-3.35)\end{array}$ & $\begin{array}{c}-0.193 * * * \\
(-3.80)\end{array}$ \\
\hline$\beta_{10}^{g}$ & & & $\begin{array}{c}-0.066^{* *} \\
(-2.16)\end{array}$ & $\begin{array}{c}-0.094 * * * \\
(-2.75)\end{array}$ & $\begin{array}{c}-0.168 * * * \\
(-3.20)\end{array}$ \\
\hline$\beta_{11}^{g}$ & & & & $\begin{array}{c}-0.099 * * * \\
(-2.95)\end{array}$ & $\begin{array}{c}-0.191 * * * \\
(-3.42)\end{array}$ \\
\hline$\beta_{12}^{g}$ & & & & & $\begin{array}{c}-0.155^{* *} \\
(-2.48)\end{array}$ \\
\hline$d_{p r e}^{g}$ & $\begin{array}{l}0.011 \\
(0.75)\end{array}$ & $\begin{array}{l}0.004 \\
(0.33)\end{array}$ & $\begin{array}{l}0.003 \\
(0.27)\end{array}$ & $\begin{array}{l}-0.022 \\
(-1.53)\end{array}$ & $\begin{array}{l}-0.028 \\
(-1.38)\end{array}$ \\
\hline$d_{\text {announce }}^{g}$ & $\begin{array}{l}0.003 \\
(0.14)\end{array}$ & $\begin{array}{l}0.006 \\
(0.29)\end{array}$ & $\begin{array}{l}-0.000 \\
(-0.01)\end{array}$ & $\begin{array}{l}-0.036 \\
(-1.48)\end{array}$ & $\begin{array}{c}-0.084^{* *} \\
(-2.14)\end{array}$ \\
\hline$d_{\text {withdrawal }}^{g}$ & $\begin{array}{c}-0.094^{* * *} \\
(-3.24)\end{array}$ & $\begin{array}{c}-0.081^{* * *} \\
(-3.28)\end{array}$ & $\begin{array}{c}-0.068^{* *} \\
(-2.39)\end{array}$ & $\begin{array}{c}-0.110^{* * *} \\
(-3.67)\end{array}$ & $\begin{array}{c}-0.183 * * * \\
(-3.67)\end{array}$ \\
\hline
\end{tabular}

Fixed-effects

Individual, year-month

$\begin{array}{llllll}\text { Observations } & 600,943 & 1,017,483 & 1,067,932 & 1,137,881 & 385,370\end{array}$

Table 3.6: The Total Debt Response Dynamics

Notes: This table shows the total debt response dynamics (equation (3)) in the period from from 2016:05 to $2017: 12$ using the matched sample. $\beta_{s}^{g}$ measures the coefficient estimates of variable $\$ W_{i}^{g} \times 1_{\text {month } s}$ and represents the average monthly response in month $s=\{-1,0, \ldots, 12\}$, where $s=-6$ stands for 2016:06,..., $s=0$ is the announcement month $2016: 12, \ldots$, and $s=12$ is the last month in the withdrawal period of group $g=5,2017: 12$. We define $d_{p r e}^{g} \equiv \frac{1}{6} \sum_{t=-6}^{-1} \beta_{t}^{g}$ as the average total debt reponse within the pre-treatment window for group $g$, where $g=\{1,2,3,4,5\}$. We decompose post-treatment period into announcement and withdrawal periods. $d_{\text {announce }}^{g} \equiv \frac{1}{\tau^{g}-1} \sum_{t=0}^{\tau^{g}-1} \beta_{t}^{g}$, where $\tau^{g}=\{3,4,5,6,7\}$ is the initial month of withdrawals release for groups $g=\{1,2,3,4,5\}$, respectively, is the average total debt response of the program in group $g$ within the announcement window. $d_{\text {withdraw }}^{g} \equiv \frac{1}{S^{g}-\left(\tau^{g}-1\right)} \sum_{t=\tau^{g}}^{S^{g}} \beta_{t}^{g}$, in turn, is the average total debt response of the program in group $g$ within the withdrawal period, where $S^{g}=\{7,8,9,10,11,12\}$ for group $g=\{1,2,3,4,5\}$, respectively. Withdrawal windows for each group are in bold. Individual and year-month fixed effects are included, and standard errors are clustered at the individual level. T-statistics are reported in parentheses under the coefficient estimates. ${ }^{* * *}$ Significant at the 1 percent level. ${ }^{* *}$ Significant at the 5 percent level. * Significant at the 10 percent level. 
Chapter 3. Follow the Money: The Effects of Liquidity Shocks on Consumption and Debt -

\begin{tabular}{|c|c|c|c|c|c|}
\hline & $\begin{array}{c}\text { Group } 1 \\
\text { (A) }\end{array}$ & $\begin{array}{c}\text { Group } 2 \\
\text { (B) }\end{array}$ & $\begin{array}{c}\text { Group } 3 \\
\text { (C) }\end{array}$ & $\begin{array}{c}\text { Group } 4 \\
\text { (D) }\end{array}$ & $\begin{array}{c}\text { Group } 5 \\
\text { (E) }\end{array}$ \\
\hline \multicolumn{6}{|l|}{ Panel A } \\
\hline$\$ W^{g} \times 1_{\text {pre }}$ & $\begin{array}{l}0.005 \\
(0.84)\end{array}$ & $\begin{array}{l}0.003 \\
(0.45)\end{array}$ & $\begin{array}{c}0.009^{* *} \\
(2.10)\end{array}$ & $\begin{array}{l}0.004 \\
(0.72)\end{array}$ & $\begin{array}{l}0.009 \\
(1.00)\end{array}$ \\
\hline$\$ W^{g} \times 1_{\text {post }}$ & $\begin{array}{c}0.052^{* * *} \\
(7.67)\end{array}$ & $\begin{array}{c}0.054 * * * \\
(7.33)\end{array}$ & $\begin{array}{c}0.051 * * * \\
(9.53)\end{array}$ & $\begin{array}{c}0.042 * * * \\
(6.43)\end{array}$ & $\begin{array}{c}0.050^{* * *} \\
(5.20)\end{array}$ \\
\hline Constant & $\begin{array}{c}2,186.500^{* * * *} \\
(169.24)\end{array}$ & $\begin{array}{c}2,201.599 * * * \\
(202.30)\end{array}$ & $\begin{array}{c}2,168.873 * * * \\
(178.57)\end{array}$ & $\begin{array}{c}2,149.537 * * * \\
(186.94)\end{array}$ & $\begin{array}{c}2,092.526 * * * \\
(136.27)\end{array}$ \\
\hline$R^{2}$ & 0.39 & 0.37 & 0.38 & 0.38 & 0.37 \\
\hline \multicolumn{6}{|l|}{ Panel B } \\
\hline$\$ W^{g} \times 1_{\text {pre }}$ & $\begin{array}{l}0.005 \\
(0.84)\end{array}$ & $\begin{array}{l}0.003 \\
(0.45)\end{array}$ & $\begin{array}{c}0.009^{* *} \\
(2.10)\end{array}$ & $\begin{array}{l}0.004 \\
(0.72)\end{array}$ & $\begin{array}{l}0.009 \\
(1.00)\end{array}$ \\
\hline$\$ W^{g} \times 1_{\text {announce }}^{g}$ & $\begin{array}{c}0.024 * * * \\
(3.24)\end{array}$ & $\begin{array}{c}0.023 * * * \\
(3.21)\end{array}$ & $\begin{array}{c}0.023 * * * \\
(4.35)\end{array}$ & $\begin{array}{c}0.019 * * * \\
(3.04)\end{array}$ & $\begin{array}{c}0.023 * * * \\
(2.57)\end{array}$ \\
\hline$\$ W^{g} \times 1_{\text {withdraw }}^{g}$ & $\begin{array}{c}0.067^{* * * *} \\
(8.85)\end{array}$ & $\begin{array}{c}0.074 * * * \\
(8.76)\end{array}$ & $\begin{array}{c}0.074 * * * \\
(11.91)\end{array}$ & $\begin{array}{c}0.064 * * * \\
(8.71)\end{array}$ & $\begin{array}{c}0.082^{* * *} \\
(5.88)\end{array}$ \\
\hline Constant & $\begin{array}{c}2,186.506^{* * *} \\
(169.24)\end{array}$ & $\begin{array}{c}2,201.608^{* * *} \\
\quad(202.30)\end{array}$ & $\begin{array}{c}2,168.883^{* * *} \\
\quad(178.57)\end{array}$ & $\begin{array}{c}2,149.550^{* * *} \\
(186.94)\end{array}$ & $\begin{array}{c}2,092.546^{* * *} \\
\quad(136.28)\end{array}$ \\
\hline$R^{2}$ & 0.39 & 0.37 & 0.38 & 0.38 & 0.37 \\
\hline Fixed effects & \multicolumn{5}{|c|}{ Individual, year-month } \\
\hline Observations & $2,574,684$ & $4,481,632$ & $4,626,813$ & $4,786,454$ & $1,633,065$ \\
\hline
\end{tabular}

Table 3.7: The Average Consumption Response to the FGTS Program

Notes: This table shows the average consumption response (equations (1) and (2)) in the period from from 2016:05 to 2017:12 using the full sample. Panel A presents the estimation results of equation (1), and panel B shows the estimation results of equation (2). $\$ W^{g}$ is the amount withdrawn by the treatment group $g$, and is equal to 0 for the control group. $1_{\text {pre }}$ is a binary variable equal to 1 for the month before the announcement (i.e., 2016:05-2016:11). $1_{\text {post }}$ is a binary variable equal to 1 for the months after the announcement of the

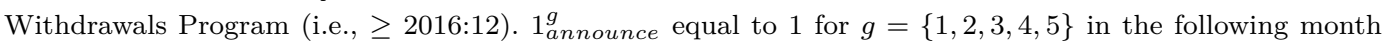
ranges: $2016: 12$ to $2017: 02,2016: 12$ to 2017:03, 2016:12 to 2017:04, 2016:12 to 2017:05, 2016:12 to 2017:06, respectively. $1_{\text {withdraw }}^{g}$ equal to 1 for $g=\{1,2,3,4,5\}$ in the following month ranges: 2016:03 to 2017:08, 2016:04 to 2017:09, 2016:05 to 2017:10, 2016:06 to 2017:11, 2016:07 to 2017:12, respectively. Individual and year-month fixed effects are included, and standard errors are clustered at the individual level. T-statistics are reported in parentheses under the coefficient estimates. $* * *$ Significant at the 1 percent level. ** Significant at the 5 percent level. * Significant at the 10 percent level. 
Chapter 3. Follow the Money: The Effects of Liquidity Shocks on Consumption and Debt -

\begin{tabular}{|c|c|c|c|c|c|}
\hline & $\begin{array}{c}\text { Group } 1 \\
\text { (A) }\end{array}$ & $\begin{array}{c}\text { Group } 2 \\
\text { (B) }\end{array}$ & $\begin{array}{c}\text { Group } 3 \\
\text { (C) }\end{array}$ & $\begin{array}{c}\text { Group } 4 \\
\text { (D) }\end{array}$ & $\begin{array}{c}\text { Group } 5 \\
\text { (E) }\end{array}$ \\
\hline \multicolumn{6}{|l|}{ Panel $A$} \\
\hline$\$ W^{g} \times 1_{\text {pre }}$ & $\begin{array}{l}0.009 \\
(1.52)\end{array}$ & $\begin{array}{l}-0.006 \\
(-1.08)\end{array}$ & $\begin{array}{l}0.004 \\
(0.73)\end{array}$ & $\begin{array}{l}-0.004 \\
(-0.57)\end{array}$ & $\begin{array}{l}-0.003 \\
(-0.37)\end{array}$ \\
\hline$\$ W^{g} \times 1_{\text {post }}$ & $\begin{array}{c}-0.039 * * * \\
(-3.39)\end{array}$ & $\begin{array}{c}-0.058^{* * *} \\
(-5.81)\end{array}$ & $\begin{array}{c}-0.033 * * * \\
(-3.05)\end{array}$ & $\begin{array}{c}-0.033^{* * *} \\
(-3.25)\end{array}$ & $\begin{array}{c}-0.044 * * * \\
(-2.57)\end{array}$ \\
\hline Constant & $\begin{array}{c}6,289.472 * * * \\
(390.51)\end{array}$ & $\begin{array}{c}6,373.876^{* * *} \\
(470.94)\end{array}$ & $\begin{array}{c}6,326.248^{* * *} \\
(462.96)\end{array}$ & $\begin{array}{c}6,263.145^{* * *} \\
(436.83)\end{array}$ & $\begin{array}{c}6,268.153 * * * \\
(244.31)\end{array}$ \\
\hline$R^{2}$ & 0.89 & 0.88 & 0.88 & 0.88 & 0.85 \\
\hline \multicolumn{6}{|l|}{ Panel B } \\
\hline$\$ W^{g} \times 1_{\text {pre }}$ & $\begin{array}{l}0.009 \\
(1.53)\end{array}$ & $\begin{array}{l}-0.006 \\
(-1.08)\end{array}$ & $\begin{array}{l}0.004 \\
(0.73)\end{array}$ & $\begin{array}{l}-0.004 \\
(-0.57)\end{array}$ & $\begin{array}{l}-0.003 \\
(-0.36)\end{array}$ \\
\hline$\$ W^{g} \times 1_{\text {announce }}^{g}$ & $\begin{array}{l}0.008 \\
(0.84)\end{array}$ & $\begin{array}{r}-0.017^{*} \\
(-1.78)\end{array}$ & $\begin{array}{l}-0.003 \\
(-0.33)\end{array}$ & $\begin{array}{l}-0.003 \\
(-0.31)\end{array}$ & $\begin{array}{l}-0.006 \\
(-0.37)\end{array}$ \\
\hline$\$ W^{g} \times 1_{\text {withdraw }}^{g}$ & $\begin{array}{c}-0.063^{* * * *} \\
(-4.82)\end{array}$ & $\begin{array}{c}-0.086^{* * *} \\
(-7.78)\end{array}$ & $\begin{array}{c}-0.058 * * * \\
(-4.63)\end{array}$ & $\begin{array}{c}-0.064 * * * \\
(-5.21)\end{array}$ & $\begin{array}{c}-0.089 * * * \\
(-4.32)\end{array}$ \\
\hline Constant & $\begin{array}{c}6,289.462 * * * \\
(390.51)\end{array}$ & $\begin{array}{c}6,373.864 * * * \\
(470.94)\end{array}$ & $\begin{array}{c}6,326.237 * * * \\
\quad(462.96)\end{array}$ & $\begin{array}{c}6,263.127 * * * \\
(436.83)\end{array}$ & $\begin{array}{c}6,268.125 * * * \\
(244.31)\end{array}$ \\
\hline$R^{2}$ & 0.89 & 0.88 & 0.88 & 0.88 & 0.85 \\
\hline Fixed effects & \multicolumn{5}{|c|}{ Individual, year-month } \\
\hline Observations & $2,574,684$ & $4,481,632$ & $4,626,813$ & $4,786,454$ & $1,633,065$ \\
\hline
\end{tabular}

Table 3.8: The Average Total Debt Response to the FGTS Program

Notes: This table shows the average total debt response (equations (1) and (2)) in the period from from 2016:05 to 2017:12 using the full sample. Panel A presents the estimation results of equation (1), and panel B shows the estimation results of equation (2). $\$ W^{g}$ is the amount withdrawn by the treatment group $g$, and is equal to 0 for the control group. $1_{\text {pre }}$ is a binary variable equal to 1 for the month before the announcement (i.e., 2016:05-2016:11). $1_{\text {post }}$ is a binary variable equal to 1 for the months after the announcement of the

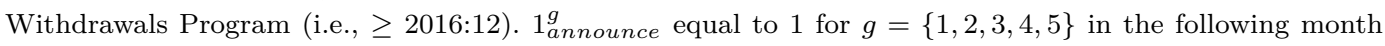
ranges: $2016: 12$ to $2017: 02,2016: 12$ to $2017: 03,2016: 12$ to $2017: 04,2016: 12$ to 2017:05, 2016:12 to 2017:06, respectively. $1_{\text {withdraw }}^{g}$ equal to 1 for $g=\{1,2,3,4,5\}$ in the following month ranges: 2016:03 to 2017:08, 2016:04 to 2017:09, 2016:05 to 2017:10, 2016:06 to 2017:11, 2016:07 to 2017:12, respectively. Individual and year-month fixed effects are included, and standard errors are clustered at the individual level. T-statistics are reported in parentheses under the coefficient estimates. ${ }^{* * *}$ Significant at the 1 percent level. ${ }^{* *}$ Significant at the 5 percent level. $*$ Significant at the 10 percent level. 
Chapter 3. Follow the Money: The Effects of Liquidity Shocks on Consumption and Debt -

\begin{tabular}{|c|c|c|c|c|c|}
\hline & $\begin{array}{l}\text { Group } 1 \\
\text { (A) }\end{array}$ & $\begin{array}{l}\text { Group } 2 \\
\text { (B) }\end{array}$ & $\begin{array}{c}\text { Group } 3 \\
\text { (C) }\end{array}$ & $\begin{array}{l}\text { Group } 4 \\
\text { (D) }\end{array}$ & $\begin{array}{c}\text { Group } 5 \\
\text { (E) }\end{array}$ \\
\hline$\beta_{0}^{g}$ & $\begin{array}{c}0.026 * * * \\
(2.70)\end{array}$ & $\begin{array}{l}0.010 \\
(1.38)\end{array}$ & $\begin{array}{c}0.014 * * \\
(2.24)\end{array}$ & $\begin{array}{l}-0.001 \\
(-0.09)\end{array}$ & $\begin{array}{l}0.004 \\
(0.42)\end{array}$ \\
\hline$\beta_{1}^{g}$ & $\begin{array}{c}0.024 * * * \\
(2.81)\end{array}$ & $\begin{array}{c}0.024 * * * \\
\quad(3.27)\end{array}$ & $\begin{array}{c}0.030 * * * \\
(4.49)\end{array}$ & $\begin{array}{c}0.021 * * * \\
(3.06)\end{array}$ & $\begin{array}{c}0.036 * * \\
(2.36)\end{array}$ \\
\hline$\beta_{2}^{g}$ & $\begin{array}{c}0.023 * * \\
(2.26)\end{array}$ & $\begin{array}{c}0.015^{* *} \\
(1.97)\end{array}$ & $\begin{array}{c}0.019 * * \\
(2.48)\end{array}$ & $\begin{array}{c}0.016^{* *} \\
(1.96)\end{array}$ & $\begin{array}{c}0.008 \\
(0.78)\end{array}$ \\
\hline$\beta_{3}^{g}$ & $\begin{array}{c}0.052 * * * \\
(3.76)\end{array}$ & $\begin{array}{c}0.040 * * * \\
(3.46)\end{array}$ & $\begin{array}{c}0.027 * * * \\
(4.09)\end{array}$ & $\begin{array}{c}0.019 * * * \\
(2.61)\end{array}$ & $\begin{array}{c}0.030^{*} \\
(1.93)\end{array}$ \\
\hline$\beta_{4}^{g}$ & $\begin{array}{c}0.043 * * * \\
(5.11)\end{array}$ & $\begin{array}{c}0.056 * * * \\
(5.30)\end{array}$ & $\begin{array}{c}0.027 * * * \\
(4.15)\end{array}$ & $\begin{array}{c}0.021 * * * \\
(2.96)\end{array}$ & $\begin{array}{l}0.012 \\
(1.23)\end{array}$ \\
\hline$\beta_{5}^{g}$ & $\begin{array}{c}0.087 * * * \\
(5.14)\end{array}$ & $\begin{array}{c}0.081 * * * \\
(7.50)\end{array}$ & $\begin{array}{c}0.063 * * * \\
(5.99)\end{array}$ & $\begin{array}{c}0.040 * * * \\
(4.53)\end{array}$ & $\begin{array}{c}0.025^{*} \\
(1.93)\end{array}$ \\
\hline$\beta_{6}^{g}$ & $\begin{array}{c}0.068 * * * \\
(6.72)\end{array}$ & $\begin{array}{c}0.085 * * * \\
(5.63)\end{array}$ & $\begin{array}{c}0.077 * * * \\
(8.49)\end{array}$ & $\begin{array}{c}0.069 * * * \\
(6.00)\end{array}$ & $\begin{array}{c}0.045^{* *} \\
(2.33)\end{array}$ \\
\hline$\beta_{7}^{g}$ & $\begin{array}{c}0.081 * * * \\
(6.71)\end{array}$ & $\begin{array}{c}0.097 * * * \\
(4.42)\end{array}$ & $\begin{array}{c}0.081 * * * \\
(8.97)\end{array}$ & $\begin{array}{c}0.071 * * * \\
(7.36)\end{array}$ & $\begin{array}{c}0.059 * * * \\
(4.12)\end{array}$ \\
\hline$\beta_{8}^{g}$ & $\begin{array}{c}0.069 * * * \\
(6.99)\end{array}$ & $\begin{array}{c}0.066 * * * \\
(7.23)\end{array}$ & $\begin{array}{c}0.077 * * * \\
(9.45)\end{array}$ & $\begin{array}{c}0.051 * * * \\
(6.23)\end{array}$ & $\begin{array}{c}0.080 * * * \\
(5.33)\end{array}$ \\
\hline$\beta_{9}^{g}$ & & $\begin{array}{c}0.062 * * * \\
(6.76)\end{array}$ & $\begin{array}{c}0.070 * * * \\
(6.85)\end{array}$ & $\begin{array}{c}0.063 * * * \\
(6.25)\end{array}$ & $\begin{array}{c}0.061 * * * \\
(4.47)\end{array}$ \\
\hline$\beta_{10}^{g}$ & & & $\begin{array}{c}0.075 * * * \\
(8.44)\end{array}$ & $\begin{array}{c}0.064 * * * \\
(7.14)\end{array}$ & $\begin{array}{c}0.056 * * * \\
(4.74)\end{array}$ \\
\hline$\beta_{11}^{g}$ & & & & $\begin{array}{c}0.065 * * * \\
(7.48)\end{array}$ & $\begin{array}{c}0.065 * * * \\
(5.65)\end{array}$ \\
\hline$\beta_{12}^{g}$ & & & & & $\begin{array}{c}0.170 * * * \\
(2.80)\end{array}$ \\
\hline$c_{p r e}^{g}$ & $\begin{array}{l}0.028 \\
(0.84)\end{array}$ & $\begin{array}{l}0.015 \\
(0.45)\end{array}$ & $\begin{array}{c}0.055^{* *} \\
(2.11)\end{array}$ & $\begin{array}{l}0.024 \\
(0.72)\end{array}$ & $\begin{array}{l}0.052 \\
(1.00)\end{array}$ \\
\hline$c_{\text {announce }}^{g}$ & $\begin{array}{c}0.073 * * * \\
(3.24)\end{array}$ & $\begin{array}{c}0.090 * * * \\
(3.21)\end{array}$ & $\begin{array}{c}0.117^{* * * *} \\
(4.35)\end{array}$ & $\begin{array}{c}0.115^{* * * *} \\
(3.03)\end{array}$ & $\begin{array}{c}0.159 * * * \\
(2.57)\end{array}$ \\
\hline$c_{\text {withdrawal }}^{g}$ & $\begin{array}{c}0.400 * * * \\
(8.86)\end{array}$ & $\begin{array}{c}0.447 * * * \\
(8.76)\end{array}$ & $\begin{array}{c}0.443 * * * \\
(11.92)\end{array}$ & $\begin{array}{c}0.383 * * * \\
(8.71)\end{array}$ & $\begin{array}{c}0.490 * * * \\
\quad(5.88)\end{array}$ \\
\hline
\end{tabular}

Fixed-effects

Individual, year-month

Observations

Table 3.9: The Consumption Response Dynamics

\footnotetext{
Notes: This table shows the consumption response dynamics (equation (3)) in the period from from 2016:05 to 2017:12 using the full sample. $\beta_{s}^{g}$ measures the coefficient estimates of variable $\$ W_{i}^{g} \times 1_{\text {month } s}$ and represents the average monthly response in month $s=\{-1,0, \ldots, 12\}$, where $s=-6$ stands for 2016:06,.., $s=0$ is the announcement month $2016: 12, \ldots$, and $s=12$ is the last month in the withdrawal period of group $g=5$, 2017:12. We define $c_{p r e}^{g} \equiv \sum_{t=-6}^{-1} \beta_{t}^{g}$ as the cumulative consumption response within the pre-treatment window for group $g$, where $g=\{1,2,3,4,5\}$. We decompose post-treatment period into announcement and withdrawal periods. $c_{\text {announce }}^{g} \equiv \sum_{t=0}^{\tau^{g}-1} \beta_{t}^{g}$, where $\tau^{g}=\{3,4,5,6,7\}$ is the initial month of withdrawals release for groups $g=\{1,2,3,4,5\}$, respectively, is the cumulative consumption response of the program in group $g$ within the announcement window. $c_{\text {withdraw }}^{g} \equiv \sum_{t=\tau}^{S^{g}} \beta_{t}^{g}$, in turn, is the cumulative consumption response of the program in group $g$ within the withdrawal period, where $S^{g}=\{7,8,9,10,11,12\}$ for group $g=\{1,2,3,4,5\}$, respectively. Withdrawal windows for each group are in bold. Individual and year-month fixed effects are included, and standard errors are clustered at the individual level. T-statistics are reported in parentheses under the coefficient estimates. ${ }^{* * *}$ Significant at the 1 percent level. ${ }^{* *}$ Significant at the 5 percent level. * Significant at the 10 percent level.
} 
Chapter 3. Follow the Money: The Effects of Liquidity Shocks on Consumption and Debt -

\begin{tabular}{|c|c|c|c|c|c|}
\hline & $\begin{array}{l}\text { Group } 1 \\
\text { (A) }\end{array}$ & $\begin{array}{l}\text { Group } 2 \\
\text { (B) }\end{array}$ & $\begin{array}{c}\text { Group } 3 \\
\text { (C) }\end{array}$ & $\begin{array}{l}\text { Group } 4 \\
\text { (D) }\end{array}$ & $\begin{array}{c}\text { Group } 5 \\
\text { (E) }\end{array}$ \\
\hline$\beta_{0}^{g}$ & $\begin{array}{l}0.001 \\
(0.05)\end{array}$ & $\begin{array}{l}-0.007 \\
(-0.31)\end{array}$ & $\begin{array}{l}-0.008 \\
(-0.93)\end{array}$ & $\begin{array}{l}-0.010 \\
(-1.19)\end{array}$ & $\begin{array}{l}-0.012 \\
(-0.86)\end{array}$ \\
\hline$\beta_{1}^{g}$ & $\begin{array}{l}0.010 \\
(0.91)\end{array}$ & $\begin{array}{l}0.006 \\
(0.25)\end{array}$ & $\begin{array}{l}-0.004 \\
(-0.36)\end{array}$ & $\begin{array}{l}-0.005 \\
(-0.51)\end{array}$ & $\begin{array}{l}-0.008 \\
(-0.50)\end{array}$ \\
\hline$\beta_{2}^{g}$ & $\begin{array}{l}0.015 \\
(1.26)\end{array}$ & $\begin{array}{l}0.017 \\
(0.80)\end{array}$ & $\begin{array}{l}-0.004 \\
(-0.44)\end{array}$ & $\begin{array}{l}-0.006 \\
(-0.61)\end{array}$ & $\begin{array}{l}0.001 \\
(0.08)\end{array}$ \\
\hline$\beta_{3}^{g}$ & $\begin{array}{c}-0.058 * * * \\
(-4.82)\end{array}$ & $\begin{array}{l}0.008 \\
(0.37)\end{array}$ & $\begin{array}{l}-0.004 \\
(-0.35)\end{array}$ & $\begin{array}{l}-0.001 \\
(-0.05)\end{array}$ & $\begin{array}{l}0.000 \\
(0.02)\end{array}$ \\
\hline$\beta_{4}^{g}$ & $\begin{array}{c}-0.074 * * * \\
(-5.74)\end{array}$ & $\begin{array}{c}-0.076 * * * \\
(-3.29)\end{array}$ & $\begin{array}{l}0.004 \\
(0.33)\end{array}$ & $\begin{array}{l}0.001 \\
(0.07)\end{array}$ & $\begin{array}{l}-0.003 \\
(-0.17)\end{array}$ \\
\hline$\beta_{5}^{g}$ & $\begin{array}{c}-0.074 * * * \\
(-5.57)\end{array}$ & $\begin{array}{c}-0.096 * * * \\
(-3.96)\end{array}$ & $\begin{array}{c}-0.07 * * * \\
(-5.96)\end{array}$ & $\begin{array}{l}0.003 \\
(0.31)\end{array}$ & $\begin{array}{l}-0.013 \\
(-0.64)\end{array}$ \\
\hline$\beta_{6}^{g}$ & $\begin{array}{c}-0.067 * * * \\
(-4.76)\end{array}$ & $\begin{array}{c}-0.097 * * * \\
(-3.82)\end{array}$ & $\begin{array}{c}-0.069 * * * \\
(-5.44)\end{array}$ & $\begin{array}{c}-0.073 * * * \\
(-6.19)\end{array}$ & $\begin{array}{l}-0.008 \\
(-0.39)\end{array}$ \\
\hline$\beta_{7}^{g}$ & $\begin{array}{c}-0.061 * * * \\
(-4.11)\end{array}$ & $\begin{array}{c}-0.086 * * * \\
(-3.29)\end{array}$ & $\begin{array}{c}-0.061 * * * \\
(-4.73)\end{array}$ & $\begin{array}{c}-0.080 * * * \\
(-6.62)\end{array}$ & $\begin{array}{c}-0.091 * * * \\
(-4.49)\end{array}$ \\
\hline$\beta_{8}^{g}$ & $\begin{array}{c}-0.045 * * * \\
(-2.90)\end{array}$ & $\begin{array}{c}-0.070 * * \\
(-2.53)\end{array}$ & $\begin{array}{c}-0.058 * * * \\
(-4.48)\end{array}$ & $\begin{array}{c}-0.071 * * * \\
(-5.57)\end{array}$ & $\begin{array}{c}-0.094 * * * \\
(-4.64)\end{array}$ \\
\hline$\beta_{9}^{g}$ & & $\begin{array}{c}-0.060 * * \\
(-2.06)\end{array}$ & $\begin{array}{c}-0.051 * * * \\
(-3.83)\end{array}$ & $\begin{array}{c}-0.061 * * * \\
(-4.76)\end{array}$ & $\begin{array}{c}-0.099 * * * \\
(-4.69)\end{array}$ \\
\hline$\beta_{10}^{g}$ & & & $\begin{array}{c}-0.037 * * * \\
(-2.72)\end{array}$ & $\begin{array}{c}-0.047 * * * \\
(-3.55)\end{array}$ & $\begin{array}{c}-0.092 * * * \\
(-4.28)\end{array}$ \\
\hline$\beta_{11}^{g}$ & & & & $\begin{array}{c}-0.050 * * * \\
(-3.70)\end{array}$ & $\begin{array}{c}-0.085 * * * \\
(-3.70)\end{array}$ \\
\hline$\beta_{12}^{g}$ & & & & & $\begin{array}{c}-0.074 * * * \\
(-3.07)\end{array}$ \\
\hline$d_{p r e}^{g}$ & $\begin{array}{l}0.009 \\
(1.53)\end{array}$ & $\begin{array}{l}-0.006 \\
(-1.08)\end{array}$ & $\begin{array}{l}0.004 \\
(0.73)\end{array}$ & $\begin{array}{l}-0.004 \\
(-0.57)\end{array}$ & $\begin{array}{l}-0.003 \\
(-0.37)\end{array}$ \\
\hline$d_{\text {announce }}^{g}$ & $\begin{array}{l}0.008 \\
(0.84)\end{array}$ & $\begin{array}{c}-0.017 * \\
(-1.78)\end{array}$ & $\begin{array}{l}-0.003 \\
(-0.33)\end{array}$ & $\begin{array}{l}-0.003 \\
(-0.31)\end{array}$ & $\begin{array}{l}-0.006 \\
(-0.37)\end{array}$ \\
\hline$d_{\text {withdrawal }}^{g}$ & $\begin{array}{c}-0.063 * * * \\
(-4.82)\end{array}$ & $\begin{array}{c}-0.086^{* * *} \\
(-7.78)\end{array}$ & $\begin{array}{c}-0.058 * * * \\
(-4.62)\end{array}$ & $\begin{array}{c}-0.064 * * * \\
(-5.21)\end{array}$ & $\begin{array}{c}-0.089 * * * \\
(-4.31)\end{array}$ \\
\hline
\end{tabular}

Fixed-effects

Individual, year-month

$\begin{array}{llllll}\text { Observations } \quad 2,574,684 & 4,481,632 & 4,626,813 & 4,626,813 & 1,633,065\end{array}$

Table 3.10: The Total Debt Response Dynamics

Notes: This table shows the total debt response dynamics (equation (3)) in the period from from 2016:05 to $2017: 12$ using the full sample. $\beta_{s}^{g}$ measures the coefficient estimates of variable $\$ W_{i}^{g} \times 1_{\text {months }}$ and represents the average monthly response in month $s=\{-1,0, \ldots, 12\}$, where $s=-6$ stands for 2016:06,..., $s=0$ is the announcement month $2016: 12, \ldots$, and $s=12$ is the last month in the withdrawal period of group $g=5,2017: 12$. We define $d_{p r e}^{g} \equiv \frac{1}{6} \sum_{t=-6}^{-1} \beta_{t}^{g}$ as the average total debt reponse within the pre-treatment window for group $g$, where $g=\{1,2,3,4,5\}$. We decompose post-treatment period into announcement and withdrawal periods. $d_{\text {announce }}^{g} \equiv \frac{1}{\tau^{g}-1} \sum_{t=0}^{\tau^{g}-1} \beta_{t}^{g}$, where $\tau^{g}=\{3,4,5,6,7\}$ is the initial month of withdrawals release for groups $g=\{1,2,3,4,5\}$, respectively, is the average total debt response of the program in group $g$ within the announcement window. $d_{\text {withdraw }}^{g} \equiv \frac{1}{S^{g}-\left(\tau^{g}-1\right)} \sum_{t=\tau^{g}}^{S^{g}} \beta_{t}^{g}$, in turn, is the average total debt response of the program in group $g$ within the withdrawal period, where $S^{g}=\{7,8,9,10,11,12\}$ for group $g=\{1,2,3,4,5\}$, respectively. Withdrawal windows for each group are in bold. Individual and year-month fixed effects are included, and standard errors are clustered at the individual level. T-statistics are reported in parentheses under the coefficient estimates. ${ }^{* * *}$ Significant at the 1 percent level. ** Significant at the 5 percent level. * Significant at the 10 percent level. 
Chapter 3. Follow the Money: The Effects of Liquidity Shocks on Consumption and Debt -

\begin{tabular}{|c|c|c|c|c|c|c|c|c|c|}
\hline & \multicolumn{3}{|c|}{$\begin{array}{l}\text { Group } 1 \\
\text { (A) }\end{array}$} & \multicolumn{3}{|c|}{$\begin{array}{l}\text { Group } 2 \\
\text { (B) }\end{array}$} & \multicolumn{3}{|c|}{$\begin{array}{c}\text { Group } 3 \\
\text { (C) }\end{array}$} \\
\hline & $\begin{array}{c}\text { Credit Card } \\
\text { Spending }\end{array}$ & Payroll Deb & $\begin{array}{c}\text { Overdraft } \\
\text { Debt }\end{array}$ & $\begin{array}{c}\text { Credit Card } \\
\text { Spending }\end{array}$ & Payroll Deb & $\begin{array}{c}\text { Overdraft } \\
\text { Debt }\end{array}$ & $\begin{array}{c}\text { Credit Card } \\
\text { Spending }\end{array}$ & Payroll Debt & $\begin{array}{c}\text { Overdraft } \\
\text { Debt }\end{array}$ \\
\hline$\beta_{0}^{g}$ & $\begin{array}{c}0.032 * * * \\
(3.21)\end{array}$ & $\begin{array}{l}0.013 \\
(0.49)\end{array}$ & $\begin{array}{l}-0.001 \\
(-0.15)\end{array}$ & $\begin{array}{l}0.003 \\
(0.40)\end{array}$ & $\begin{array}{l}-0.003 \\
(-0.16)\end{array}$ & $\begin{array}{l}-0.002 \\
(-0.31)\end{array}$ & $\begin{array}{c}0.017^{* *} \\
(2.04)\end{array}$ & $\begin{array}{l}0.032 \\
(1.36)\end{array}$ & $\begin{array}{l}-0.004 \\
(-0.77)\end{array}$ \\
\hline$\beta_{1}^{g}$ & $\begin{array}{c}0.042 * * * \\
(3.91)\end{array}$ & $\begin{array}{l}0.027 \\
(0.92)\end{array}$ & $\begin{array}{l}0.011^{*} \\
(1.70)\end{array}$ & $\begin{array}{l}0.011 \\
(1.28)\end{array}$ & $\begin{array}{l}-0.003 \\
(-0.19)\end{array}$ & $\begin{array}{l}0.004 \\
(0.60)\end{array}$ & $\begin{array}{c}0.022^{* *} \\
(2.34)\end{array}$ & $\begin{array}{l}0.017 \\
(0.69)\end{array}$ & $\begin{array}{l}0.006 \\
(1.19)\end{array}$ \\
\hline$\beta_{2}^{g}$ & $\begin{array}{c}0.032 * * * \\
(3.26)\end{array}$ & $\begin{array}{l}0.020 \\
(0.65)\end{array}$ & $\begin{array}{l}0.012 \\
(1.55)\end{array}$ & $\begin{array}{l}0.009 \\
(1.08)\end{array}$ & $\begin{array}{l}-0.022 \\
(-1.12)\end{array}$ & $\begin{array}{l}0.004 \\
(0.60)\end{array}$ & $\begin{array}{c}0.017 * \\
(1.85)\end{array}$ & $\begin{array}{l}0.002 \\
(0.06)\end{array}$ & $\begin{array}{l}0.001 \\
(0.25)\end{array}$ \\
\hline$\beta_{3}^{g}$ & $\begin{array}{c}0.026^{* *} \\
(2.55)\end{array}$ & $\begin{array}{c}-0.058^{*} \\
(-1.82)\end{array}$ & $\begin{array}{c}-0.027^{* * *} \\
(-4.2)\end{array}$ & $\begin{array}{c}0.022 * * * \\
(2.58)\end{array}$ & $\begin{array}{c}-0.038^{*} \\
(-1.82)\end{array}$ & $\begin{array}{c}0.013^{* *} \\
(1.97)\end{array}$ & $\begin{array}{c}0.022^{* *} \\
(2.36)\end{array}$ & $\begin{array}{l}-0.010 \\
(-0.39)\end{array}$ & $\begin{array}{l}0.007 \\
(1.15)\end{array}$ \\
\hline$\beta_{4}^{g}$ & $\begin{array}{c}0.045^{* * * *} \\
(4.06)\end{array}$ & $\begin{array}{c}-0.082^{* *} \\
(-2.46)\end{array}$ & $\begin{array}{c}-0.018^{* *} \\
(-2.49)\end{array}$ & $\begin{array}{c}0.024^{* * *} \\
(2.69)\end{array}$ & $\begin{array}{c}-0.087^{* * *} \\
(-4.03)\end{array}$ & $\begin{array}{c}-0.034^{* * *} \\
(-4.80)\end{array}$ & $\begin{array}{c}0.025^{* * *} \\
(2.66)\end{array}$ & $\begin{array}{l}-0.018 \\
(-0.66)\end{array}$ & $\begin{array}{c}0.020^{* * *} \\
(2.80)\end{array}$ \\
\hline$\beta_{5}^{g}$ & $\begin{array}{c}0.056^{* * *} \\
(5.03)\end{array}$ & $\begin{array}{c}-0.099 * * * \\
(-2.86)\end{array}$ & $\begin{array}{l}-0.011 \\
(-1.51)\end{array}$ & $\begin{array}{c}0.033^{* * *} \\
(3.55)\end{array}$ & $\begin{array}{c}-0.111 * * * \\
(-4.87)\end{array}$ & $\begin{array}{c}-0.018 \text { *** } \\
(-2.57)\end{array}$ & $\begin{array}{c}0.033 * * * \\
(3.55)\end{array}$ & $\begin{array}{c}-0.085^{* * *} \\
(-3.06)\end{array}$ & $\begin{array}{c}-0.031 * * * \\
(-4.68)\end{array}$ \\
\hline$\beta_{6}^{g}$ & $\begin{array}{c}0.058^{* * *} \\
(5.09)\end{array}$ & $\begin{array}{c}-0.080^{* *} \\
(-2.09)\end{array}$ & $\begin{array}{l}-0.001 \\
(-0.18)\end{array}$ & $\begin{array}{c}0.042 * * * \\
(3.79)\end{array}$ & $\begin{array}{c}-0.107^{* * *} \\
(-4.40)\end{array}$ & $\begin{array}{c}-0.017^{* *} \\
(-2.42)\end{array}$ & $\begin{array}{c}0.042^{\star * * *} \\
(4.41)\end{array}$ & $\begin{array}{c}-0.094^{* * *} \\
(-3.21)\end{array}$ & $\begin{array}{c}-0.028 * * * \\
(-4.93)\end{array}$ \\
\hline$\beta_{7}^{g}$ & $\begin{array}{c}0.065^{* * *} \\
(5.23)\end{array}$ & $\begin{array}{c}-0.093^{\star *} \\
(-2.39)\end{array}$ & $\begin{array}{l}0.000 \\
(0.06)\end{array}$ & $\begin{array}{c}0.047^{* * *} \\
(4.06)\end{array}$ & $\begin{array}{c}-0.114 * * * \\
(-4.53)\end{array}$ & $\begin{array}{l}-0.011 \\
(-1.62)\end{array}$ & $\begin{array}{c}0.049 * * * \\
(4.84)\end{array}$ & $\begin{array}{c}-0.088^{* * *} \\
(-2.81)\end{array}$ & $\begin{array}{c}-0.014^{* *} \\
(-2.39)\end{array}$ \\
\hline$\beta_{8}^{g}$ & $\begin{array}{c}0.056^{* * *} \\
(4.40)\end{array}$ & $\begin{array}{c}-0.083^{* *} \\
(-2.06)\end{array}$ & $\begin{array}{l}0.000 \\
(0.03)\end{array}$ & $\begin{array}{c}0.04 * * * \\
(3.35)\end{array}$ & $\begin{array}{c}-0.089^{* * * *} \\
(-3.25)\end{array}$ & $\begin{array}{l}-0.007 \\
(-1.02)\end{array}$ & $\begin{array}{c}0.049^{* * *} \\
(4.99)\end{array}$ & $\begin{array}{c}-0.082^{* * *} \\
(-2.56)\end{array}$ & $\begin{array}{l}-0.007 \\
(-1.17)\end{array}$ \\
\hline$\beta_{9}^{g}$ & & & & $\begin{array}{c}0.044^{* * *} \\
(3.77)\end{array}$ & $\begin{array}{c}-0.079 * * * \\
(-2.74)\end{array}$ & $\begin{array}{l}-0.008 \\
(-1.20)\end{array}$ & $\begin{array}{c}0.045^{* * *} \\
(4.44)\end{array}$ & $\begin{array}{c}-0.073^{* *} \\
(-2.22)\end{array}$ & $\begin{array}{l}-0.003 \\
(-0.54)\end{array}$ \\
\hline$\beta_{10}^{g}$ & & & & & & & $\begin{array}{c}0.043^{* * *} \\
(4.30)\end{array}$ & $\begin{array}{c}-0.064^{*} \\
(-1.88)\end{array}$ & $\begin{array}{l}0.003 \\
(0.52)\end{array}$ \\
\hline \multicolumn{10}{|l|}{$\beta_{11}^{g}$} \\
\hline Constant & $\begin{array}{c}2,511.572^{* * *} \\
\quad(165.18)\end{array}$ & $\begin{array}{c}11,536.33 * * * \\
\quad(241.94)\end{array}$ & $\begin{array}{c}831.671^{* * * *} \\
(90.47)\end{array}$ & $\begin{array}{c}2,562.497^{* * *} \\
(220.18)\end{array}$ & $\begin{array}{l}11,444.89^{* * *} \\
\quad(311.61)\end{array}$ & $\begin{array}{c}851.126^{* * *} \\
(111.45)\end{array}$ & $\begin{array}{c}2,483.562 * * * \\
(209.76)\end{array}$ & $\begin{array}{c}11,493.2 * * * \\
(305.73)\end{array}$ & $\begin{array}{c}836.877 * * * \\
(112.00)\end{array}$ \\
\hline$c_{\text {pre }}^{g}, d_{\text {pre }}^{g}$ & $\begin{array}{l}0.010 \\
(1.45)\end{array}$ & $\begin{array}{l}0.027 \\
(1.36)\end{array}$ & $\begin{array}{l}0.002 \\
(0.38)\end{array}$ & $\begin{array}{l}-0.027 \\
(-0.89)\end{array}$ & $\begin{array}{l}0.006 \\
(0.54)\end{array}$ & $\begin{array}{l}0.004 \\
(0.89)\end{array}$ & $\begin{array}{l}0.026 \\
(0.75)\end{array}$ & $\begin{array}{l}0.014 \\
(1.16)\end{array}$ & $\begin{array}{l}0.003 \\
(0.82)\end{array}$ \\
\hline Fixed-effects & & & & Indivi & idual, year-mon & & & & \\
\hline Observations & & 487,075 & & & 851,254 & & & $1,080,025$ & \\
\hline
\end{tabular}

Table 3.11: Credit Card Spending, Payroll Debt and Overdraft Debt Dynamics Responses - Restricted Sample 
Chapter 3. Follow the Money: The Effects of Liquidity Shocks on Consumption and Debt -

\begin{tabular}{|c|c|c|c|c|c|c|}
\hline & \multicolumn{3}{|c|}{$\begin{array}{l}\text { Group } 4 \\
\text { (D) }\end{array}$} & \multicolumn{3}{|c|}{$\begin{array}{l}\text { Group } 5 \\
\text { (E) }\end{array}$} \\
\hline & $\begin{array}{c}\text { Credit Card } \\
\text { Spending }\end{array}$ & Payroll Debt & $\begin{array}{l}\text { Overdraft } \\
\text { Debt }\end{array}$ & $\begin{array}{l}\text { Credit Card } \\
\text { Spending }\end{array}$ & Payroll Debt & $\begin{array}{l}\text { Overdraft } \\
\text { Debt }\end{array}$ \\
\hline$\beta_{0}^{g}$ & $\begin{array}{c}0.022 * * * \\
(2.66)\end{array}$ & $\begin{array}{l}0.016 \\
(0.88)\end{array}$ & $\begin{array}{l}-0.006 \\
(-1.11)\end{array}$ & $\begin{array}{l}0.002 \\
(0.14)\end{array}$ & $\begin{array}{l}0.003 \\
(0.09)\end{array}$ & $\begin{array}{l}-0.006 \\
(-0.65)\end{array}$ \\
\hline$\beta_{1}^{g}$ & $\begin{array}{c}0.023^{* * *} \\
(2.91)\end{array}$ & $\begin{array}{l}0.010 \\
(0.47)\end{array}$ & $\begin{array}{l}0.004 \\
(0.68)\end{array}$ & $\begin{array}{l}0.011 \\
(0.79)\end{array}$ & $\begin{array}{l}0.010 \\
(0.28)\end{array}$ & $\begin{array}{l}0.002 \\
(0.22)\end{array}$ \\
\hline$\beta_{2}^{g}$ & $\begin{array}{c}0.016^{* *} \\
(2.02)\end{array}$ & $\begin{array}{l}0.014 \\
(0.66)\end{array}$ & $\begin{array}{l}0.002 \\
(0.40)\end{array}$ & $\begin{array}{l}0.001 \\
(0.10)\end{array}$ & $\begin{array}{l}0.011 \\
(0.29)\end{array}$ & $\begin{array}{l}-0.009 \\
(-1.04)\end{array}$ \\
\hline$\beta_{3}^{g}$ & $\begin{array}{c}0.022 * * \\
(2.50)\end{array}$ & $\begin{array}{l}0.003 \\
(0.14)\end{array}$ & $\begin{array}{l}0.003 \\
(0.52)\end{array}$ & $\begin{array}{l}0.005 \\
(0.36)\end{array}$ & $\begin{array}{l}0.001 \\
(0.03)\end{array}$ & $\begin{array}{c}0.000 \\
(-0.02)\end{array}$ \\
\hline$\beta_{4}^{g}$ & $\begin{array}{c}0.026^{* * *} \\
(2.87)\end{array}$ & $\begin{array}{l}-0.012 \\
(-0.46)\end{array}$ & $\begin{array}{c}0.00 \\
(0.05)\end{array}$ & $\begin{array}{l}0.006 \\
(0.47)\end{array}$ & $\begin{array}{l}-0.021 \\
(-0.51)\end{array}$ & $\begin{array}{l}-0.004 \\
(-0.36)\end{array}$ \\
\hline$\beta_{5}^{g}$ & $\begin{array}{c}0.032 * * * \\
\quad(3.49)\end{array}$ & $\begin{array}{l}-0.036 \\
(-1.33)\end{array}$ & $\begin{array}{c}0.021^{* * *} \\
(2.87)\end{array}$ & $\begin{array}{l}0.008 \\
(0.56)\end{array}$ & $\begin{array}{l}-0.027 \\
(-0.61)\end{array}$ & $\begin{array}{l}0.000 \\
(0.00)\end{array}$ \\
\hline$\beta_{6}^{g}$ & $\begin{array}{c}0.036 * * * \\
(3.90)\end{array}$ & $\begin{array}{c}-0.097 * * * \\
(-3.48)\end{array}$ & $\begin{array}{c}-0.034 * * * \\
(-5.76)\end{array}$ & $\begin{array}{l}0.013 \\
(0.92)\end{array}$ & $\begin{array}{l}-0.030 \\
(-0.68)\end{array}$ & $\begin{array}{l}0.012 \\
(1.14)\end{array}$ \\
\hline$\beta_{7}^{g}$ & $\begin{array}{c}0.043 * * * \\
(4.34)\end{array}$ & $\begin{array}{c}-0.112 * * * \\
(-3.91)\end{array}$ & $\begin{array}{c}-0.019 * * * \\
(-2.68)\end{array}$ & $\begin{array}{r}0.028 * \\
(1.83)\end{array}$ & $\begin{array}{c}-0.090 * * \\
(-1.98)\end{array}$ & $\begin{array}{c}-0.035 * * * \\
(-3.61)\end{array}$ \\
\hline$\beta_{8}^{g}$ & $\begin{array}{c}0.050 * * * \\
(4.59)\end{array}$ & $\begin{array}{c}-0.114 * * * \\
(-3.89)\end{array}$ & $\begin{array}{l}-0.012 \\
(-1.51)\end{array}$ & $\begin{array}{c}0.029 * \\
(1.78)\end{array}$ & $\begin{array}{c}-0.107 * * \\
(-2.27)\end{array}$ & $\begin{array}{c}-0.028 * * * \\
(-2.95)\end{array}$ \\
\hline$\beta_{9}^{g}$ & $\begin{array}{c}0.056 * * * \\
(4.97)\end{array}$ & $\begin{array}{c}-0.115^{* * *} \\
(-3.90)\end{array}$ & $\begin{array}{l}-0.004 \\
(-0.55)\end{array}$ & $\begin{array}{c}0.034 * * \\
(2.15)\end{array}$ & $\begin{array}{c}-0.099 * * \\
(-1.96)\end{array}$ & $\begin{array}{c}-0.027 * * * \\
(-2.97)\end{array}$ \\
\hline$\beta_{10}^{g}$ & $\begin{array}{c}0.056 * * * \\
(4.86)\end{array}$ & $\begin{array}{c}-0.117 * * * \\
(-3.78)\end{array}$ & $\begin{array}{l}0.001 \\
(0.14)\end{array}$ & $\begin{array}{c}0.032 * * \\
(2.07)\end{array}$ & $\begin{array}{c}-0.102 * \\
(-1.91)\end{array}$ & $\begin{array}{l}-0.015 \\
(-1.48)\end{array}$ \\
\hline$\beta_{11}^{g}$ & $\begin{array}{c}0.059 * * * \\
(4.90)\end{array}$ & $\begin{array}{c}-0.125 * * * \\
(-3.94)\end{array}$ & $\begin{array}{l}-0.003 \\
(-0.46)\end{array}$ & $\begin{array}{c}0.043 * * * \\
(2.76)\end{array}$ & $\begin{array}{r}-0.098^{*} \\
(-1.78)\end{array}$ & $\begin{array}{r}-0.018^{*} \\
(-1.74)\end{array}$ \\
\hline$\beta_{12}^{g}$ & & & & $\begin{array}{c}0.056^{* * *} \\
(3.33)\end{array}$ & $\begin{array}{l}-0.075 \\
(-1.35)\end{array}$ & $\begin{array}{c}-0.016 * \\
(-1.72)\end{array}$ \\
\hline Constant & $\begin{array}{c}2,466.065^{* * *} \\
\quad(208.02)\end{array}$ & $\begin{array}{c}11,333.22 * * * \\
(286.35)\end{array}$ & $\begin{array}{c}820.826^{* * *} \\
\quad(110.25)\end{array}$ & $\begin{array}{c}2,522.871^{* * *} \\
\quad(118.67)\end{array}$ & $\begin{array}{c}11,251.73^{* * *} \\
(162.47)\end{array}$ & $\begin{array}{c}818.062^{* * *} \\
\quad(64.44)\end{array}$ \\
\hline$c_{p r e}^{g}, d_{\text {pre }}^{g}$ & $\begin{array}{l}0.037 \\
(1.26)\end{array}$ & $\begin{array}{l}0.019 \\
(1.61)\end{array}$ & $\begin{array}{l}-0.003 \\
(-0.76)\end{array}$ & $\begin{array}{l}0.034 \\
(0.67)\end{array}$ & $\begin{array}{l}0.026 \\
(1.09)\end{array}$ & $\begin{array}{l}-0.001 \\
(-0.11)\end{array}$ \\
\hline Fixed-effects & & & Individual & ear-month & & \\
\hline Observations & & $1,119,123$ & & & 387,794 & \\
\hline
\end{tabular}

Credit Card Spending, Payroll Debt and Overdraft Debt Dynamics

Responses - Restricted Sample (Continued)

Notes: This table shows the credit card spending, payroll debt and overdraft debt response dynamics (equation (3)) in the period from from 2016:05 to 2017:12 using the full sample, restricted to individual holding at least credit card spending and these two debt modalities. $\beta_{s}^{g}$ measures the coefficient estimates of variable $\$ W_{i}^{g} \times 1_{\text {month } s}$ and represents the average monthly response in month $s=\{-1,0, \ldots, 12\}$, where $s=-1$ stands for 2016:11, $s=0$ is the announcement month 2016:12,.., and $s=12$ is the last month in the withdrawal period of group $g=5,2017: 12$. We define $c_{p r e}^{g} \equiv \sum_{t=-6}^{-1} \beta_{t}^{g}$ and $d_{p r e}^{g} \equiv \frac{1}{6} \sum_{t=-6}^{-1} \beta_{t}^{g}$ as the cumulative consumption response and the average debt modality response, respectively, within the pre-treatment window for group $g$, where $g=\{1,2,3,4,5\}$. Withdrawal periods for each group are in bold. Individual and year-month fixed effects are included, and standard errors are clustered at the individual level. T-statistics are reported in parentheses under the coefficient estimates. ${ }^{* * *}$ Significant at the 1 percent level. ${ }^{* *}$ Significant at the 5 percent level. ${ }^{*}$ Significant at the 10 percent level. 
Chapter 3. Follow the Money: The Effects of Liquidity Shocks on Consumption and Debt -

\begin{tabular}{|c|c|c|c|c|c|c|c|c|}
\hline & \multirow{2}{*}{\multicolumn{3}{|c|}{ Consumption Proxies }} & \multicolumn{5}{|c|}{ Debt } \\
\hline & & & & \multicolumn{3}{|c|}{ Selected Debt Modalities } & \multirow[b]{2}{*}{$\begin{array}{c}\text { Total } \\
\text { Overdue } \\
\\
(\mathrm{G}) \\
\end{array}$} & \multirow[b]{2}{*}{$\begin{array}{c}\text { Total } \\
\text { Default } \\
\\
(\mathrm{H}) \\
\end{array}$} \\
\hline & $\begin{array}{c}\text { Credit Card } \\
\text { Spending } \\
\text { (A) }\end{array}$ & $\begin{array}{l}\text { Vehicles } \\
\text { Down } \\
\text { Payments } \\
\text { (B) }\end{array}$ & $\begin{array}{l}\text { Real estate } \\
\text { Down } \\
\text { Payments } \\
\text { (C) }\end{array}$ & $\begin{array}{c}\text { Credit Card } \\
\text { (D) }\end{array}$ & $\begin{array}{c}\text { Overdraft } \\
\text { (E) }\end{array}$ & $\begin{array}{l}\text { Payroll } \\
\text { (F) }\end{array}$ & & \\
\hline \multicolumn{9}{|l|}{ Panel A. Group I } \\
\hline$\$ W^{1} \times 1_{\text {post }}$ & $\begin{array}{c}0.031^{* * *} \\
(9.08)\end{array}$ & $\begin{array}{l}0.004^{* * *} \\
(3.08)\end{array}$ & $\begin{array}{c}0.014 * * * \\
(3.71)\end{array}$ & $\begin{array}{c}-0.006^{* * *} \\
(-2.58)\end{array}$ & $\begin{array}{c}-0.007 * * * \\
(-3.47)\end{array}$ & $\begin{array}{c}-0.018^{* * *} \\
(-4.27)\end{array}$ & $\begin{array}{c}-0.011^{* *} \\
(-2.21)\end{array}$ & $\begin{array}{c}-0.008^{*} \\
(-1.87)\end{array}$ \\
\hline Constant & $\begin{array}{c}2,016.257 * * * \\
\quad(339.56)\end{array}$ & $\begin{array}{c}76.609^{* * *} \\
(17.35)\end{array}$ & $\begin{array}{c}93.627^{* * * *} \\
(8.85)\end{array}$ & $\begin{array}{c}583.016^{* * *} \\
(129.49)\end{array}$ & $\begin{array}{c}390.945^{* * *} \\
(134.81)\end{array}$ & $\begin{array}{c}2,742.547^{* * * *} \\
\quad(300.69)\end{array}$ & $\begin{array}{c}802.843^{* * * *} \\
(97.17)\end{array}$ & $\begin{array}{c}544.02^{* * * *} \\
(73.32)\end{array}$ \\
\hline Fixed effects & \multicolumn{8}{|c|}{ Individual, year-month } \\
\hline $\begin{array}{l}R^{2} \\
\text { Observations }\end{array}$ & 0.84 & 0.07 & 0.07 & $\begin{array}{l}0.64 \\
2,574\end{array}$ & 0.63 & 0.92 & 0.56 & 0.52 \\
\hline \multicolumn{9}{|l|}{ Panel B. Group 2} \\
\hline$\$ W^{2} \times 1_{\text {post }}$ & $\begin{array}{c}0.032^{* * *} \\
(9.31)\end{array}$ & $\begin{array}{c}0.002^{*} \\
(1.67)\end{array}$ & $\begin{array}{c}0.018^{* * * *} \\
(5.69)\end{array}$ & $\begin{array}{c}-0.009 * * * \\
(-5.03)\end{array}$ & $\begin{array}{c}-0.007 * * * \\
(-4.49)\end{array}$ & $\begin{array}{c}-0.021 * * * \\
(-6.82)\end{array}$ & $\begin{array}{c}-0.016^{* * *} \\
(-5.04)\end{array}$ & $\begin{array}{c}-0.012 * * * \\
(-4.28)\end{array}$ \\
\hline Constant & $\begin{array}{c}2,016.137^{* * * *} \\
\quad(424.06)\end{array}$ & $\begin{array}{c}80.038^{* * *} \\
(21.15)\end{array}$ & $\begin{array}{c}105.423^{* * *} \\
(11.77)\end{array}$ & $\begin{array}{c}595.086^{* * *} \\
(153.52)\end{array}$ & $\begin{array}{c}393.164 * * * \\
(164.37)\end{array}$ & $\begin{array}{c}2,716.695^{* * *} \\
\quad(373.46)\end{array}$ & $\begin{array}{c}806.393^{* * *} \\
(119.31)\end{array}$ & $\begin{array}{c}543.099^{* * * *} \\
(89.94)\end{array}$ \\
\hline Fixed effects & \multicolumn{8}{|c|}{ Individual, year-month } \\
\hline $\begin{array}{l}R^{2} \\
\text { Observations }\end{array}$ & 0.82 & 0.06 & 0.06 & $\begin{array}{l}0.60 \\
4,481\end{array}$ & 0.62 & 0.91 & 0.53 & 0.48 \\
\hline \multicolumn{9}{|l|}{ Panel C. Group 3} \\
\hline$\$ W^{3} \times 1_{\text {post }}$ & $\begin{array}{l}0.031^{* * *} \\
(10.16)\end{array}$ & $\begin{array}{l}0.001 \\
(1.45)\end{array}$ & $\begin{array}{c}0.011^{* * *} \\
(4.72)\end{array}$ & $\begin{array}{c}-0.006^{* * *} \\
(-3.05)\end{array}$ & $\begin{array}{c}-0.006^{* * *} \\
(-5.83)\end{array}$ & $\begin{array}{c}-0.018^{* * *} \\
(-4.72)\end{array}$ & $\begin{array}{c}-0.009 * * * \\
(-2.69)\end{array}$ & $\begin{array}{c}-0.006^{* *} \\
(-2.19)\end{array}$ \\
\hline Constant & $\begin{array}{c}1,973.635^{* * *} \\
\quad(418.21)\end{array}$ & $\begin{array}{c}86.094 * * * \\
(21.29)\end{array}$ & $\begin{array}{c}109.15^{* * *} \\
(10.49)\end{array}$ & $\begin{array}{c}596.229 * * * \\
(161.98)\end{array}$ & $\begin{array}{c}387.297 * * * \\
(249.37)\end{array}$ & $\begin{array}{c}2,745.917 * * * \\
\quad(373.46)\end{array}$ & $\begin{array}{c}805.933^{* * * *} \\
(118.29)\end{array}$ & $\begin{array}{c}546.033^{* * *} \\
(90.06)\end{array}$ \\
\hline Fixed effects & & & & Individual, ye & r-month & & & \\
\hline $\begin{array}{l}R^{2} \\
\text { Observations }\end{array}$ & 0.83 & 0.06 & 0.06 & $\begin{array}{l}0.60 \\
4,626\end{array}$ & 0.56 & 0.91 & 0.52 & 0.47 \\
\hline \multicolumn{9}{|l|}{ Panel D. Group 4} \\
\hline$\$ W^{4} \times 1_{\text {post }}$ & $\begin{array}{l}0.030^{* * *} \\
(10.00)\end{array}$ & $\begin{array}{l}0.001 \\
(1.28)\end{array}$ & $\begin{array}{c}0.006^{* * *} \\
(3.16)\end{array}$ & $\begin{array}{c}-0.004 * * * \\
(-2.64)\end{array}$ & $\begin{array}{c}-0.003 * * * \\
(-2.77)\end{array}$ & $\begin{array}{c}-0.022^{* * *} \\
(-6.34)\end{array}$ & $\begin{array}{c}-0.007 * * \\
(-2.26)\end{array}$ & $\begin{array}{l}-0.003 \\
(-1.33)\end{array}$ \\
\hline Constant & $\begin{array}{c}1,969.63^{* * *} \\
(414.07)\end{array}$ & $\begin{array}{c}83.500^{* * *} \\
(15.58)\end{array}$ & $\begin{array}{c}96.408^{* * *} \\
(10.82)\end{array}$ & $\begin{array}{c}585.214^{* * *} \\
(150.51)\end{array}$ & $\begin{array}{c}381.967 * * * \\
(153.11)\end{array}$ & $\begin{array}{c}2,698.196 * * * \\
\quad(348.53)\end{array}$ & $\begin{array}{l}792.27 * * * \\
(114.53)\end{array}$ & $\begin{array}{c}538.806 * * * \\
(84.48)\end{array}$ \\
\hline Fixed effects & & & & Individual, ye & r-month & & & \\
\hline $\begin{array}{l}R^{2} \\
\text { Observations }\end{array}$ & 0.82 & 0.06 & 0.06 & $\begin{array}{l}0.58 \\
4,786\end{array}$ & 0.60 & 0.90 & 0.50 & 0.46 \\
\hline \multicolumn{9}{|l|}{ Panel E. Group 5} \\
\hline$\$ W^{5} \times 1_{\text {post }}$ & $\begin{array}{c}0.033^{* * *} \\
(6.66)\end{array}$ & $\begin{array}{c}0.003^{*} \\
(1.82)\end{array}$ & $\begin{array}{l}0.006 \\
(0.90)\end{array}$ & $\begin{array}{c}-0.007^{* *} \\
(-2.11)\end{array}$ & $\begin{array}{c}-0.004^{* * *} \\
(-2.65)\end{array}$ & $\begin{array}{c}-0.018^{* * *} \\
(-2.89)\end{array}$ & $\begin{array}{l}-0.004 \\
(-0.61)\end{array}$ & $\begin{array}{l}-0.002 \\
(-0.34)\end{array}$ \\
\hline Constant & $\begin{array}{l}1,955.593^{* * *} \\
\quad(216.54)\end{array}$ & $\begin{array}{c}73.26^{* * *} \\
(10.8)\end{array}$ & $\begin{array}{c}63.668^{* * *} \\
\quad(6.21)\end{array}$ & $\begin{array}{c}597.162^{* * *} \\
(86.89)\end{array}$ & $\begin{array}{c}381.034^{* * *} \\
(89.06)\end{array}$ & $\begin{array}{c}2,716.695^{* * *} \\
\quad(197.36)\end{array}$ & $\begin{array}{c}810.383^{* * *} \\
(62.29)\end{array}$ & $\begin{array}{c}554.208^{* * *} \\
\quad(47.07)\end{array}$ \\
\hline Fixed effects & & & & Individual, ye & -month & & & \\
\hline $\begin{array}{l}R^{2} \\
\text { Observations }\end{array}$ & 0.83 & 0.05 & 0.05 & $\begin{array}{l}0.57 \\
1,633\end{array}$ & 0.58 & 0.90 & 0.48 & 0.43 \\
\hline
\end{tabular}

Table 3.12: Response heterogeneity by consumption categories and debt modalities

Notes: This table shows the monthly average response of consumption categories and the average response of debt modalities, total overdue debt and total default debt (equation (1)) in the period from from 2016:05 to 2017:12 for each of the five groups of treated workers (Panel A to Panel E) using the full sample. $\$ W^{g}$ is the amount withdrawn by the treatment group $g, g=\{1,2,3,4,5\}$, and is equal to 0 for the control group. $1_{\text {post }}$ is a binary variable equal to 1 for the months after the announcement of the Withdrawals Program (i.e., $\geq 2016: 12$ ). Individual and year-month fixed effects are included, and standard errors are clustered at the individual level. T-statistics are reported in parentheses under the coefficient estimates. ${ }^{* * *}$ Significant at the 1 percent level. ${ }^{*}$ Significant at the 5 percent level. ${ }^{*}$ Significant at the 10 percent level. 
Chapter 3. Follow the Money: The Effects of Liquidity Shocks on Consumption and Debt -

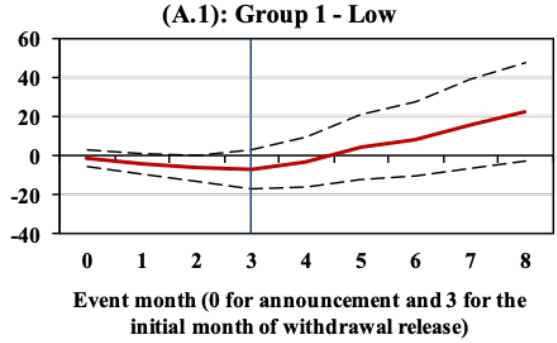

(B.1): Group 2 - Low

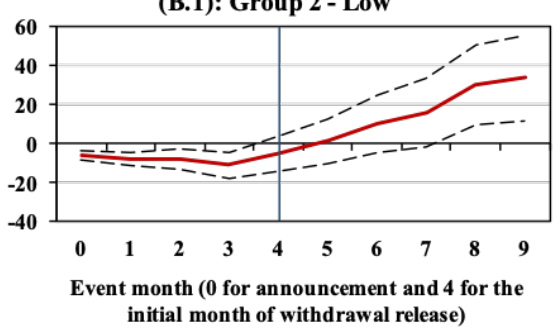

(C.1): Group 3 - Low

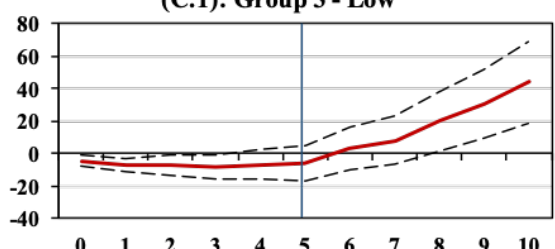

$\begin{array}{llllllllll}1 & 2 & 3 & 4 & 5 & 6 & 7 & 8 & 9 & 10\end{array}$ initial month of withdrawal release)

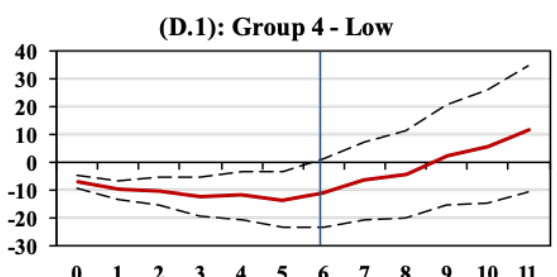

$\begin{array}{llllllllllll}0 & 1 & 2 & 3 & 4 & 5 & 6 & 7 & 8 & 9 & 10 & 11\end{array}$ initial month of withdrawal release)

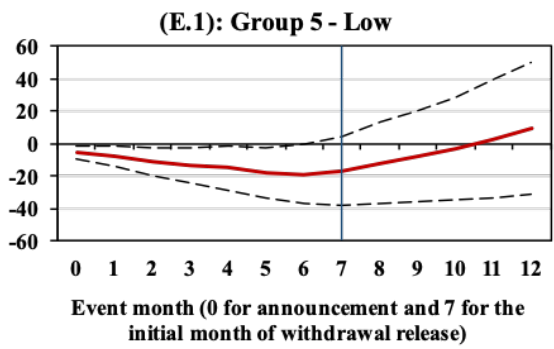

(A.2): Group 1 - High

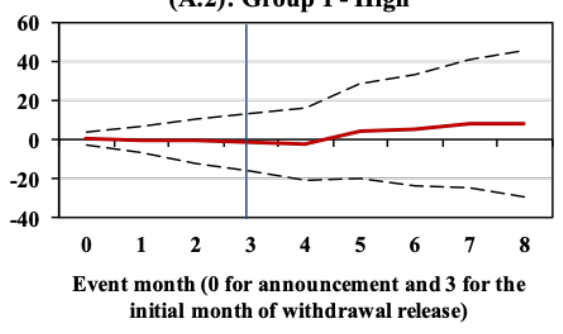

(B.2): Group 2 - High

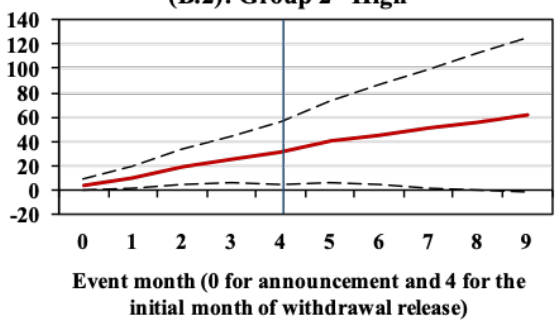

(C.2): Group 3 - High

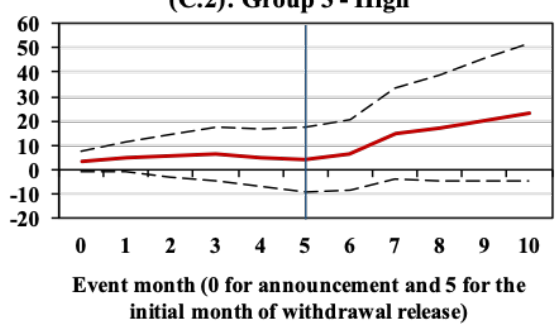

(D.2): Group 4 - High

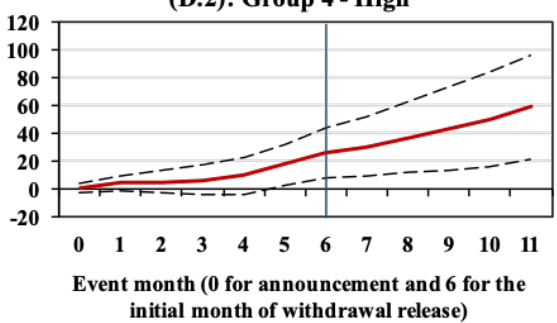

(E.2): Group 5 - High

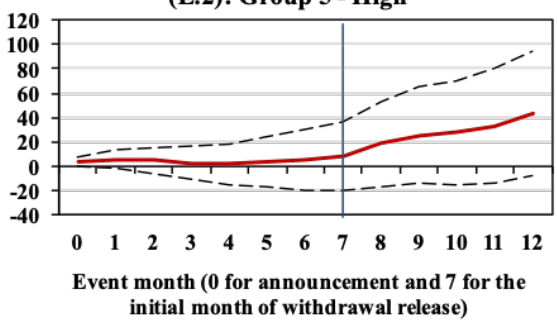

Figure 3.7: Heterogeneity in Consumption Response across Workers Types - Age

Notes: This figure plots for each group of consumers, the cumulative consumption response (starting from the announcement month $s=0$ ) up to each month in the post-treatment period in the x-axis, $c_{t} g \equiv \sum_{s=0}^{t^{g}} \beta_{s}^{g}$, where $t^{g}=\left\{0, \ldots, S^{g}\right\}$ for $g=\{1,2,3,4,5\}$, respectively, along with their corresponding 95 percent confidence intervals, across different consumers. The y-axis shows the $\$$ response (for every $\$ 100$ received). The sample includes the treatment and control groups during the period of 2016:05-2017:12. Column 1 plots workers with low age in each group (i.e., age between 2016:01 and 2016:04 $\leq 26$ or bottom tenth of the sample). Column 2 shows workers with high age in each group (i.e., age between 2016:01 and 2016:04 $\geq 54$, or top tenth of the sample). 


\section{Bibliography}

Aaronson, D., Agarwal, S., and French, E. (2012). The spending and debt response to minimum wage hikes. American Economic Review, 102(7):311139.

Acemoglu, D. and Guerrieri, V. (2006). Capital deepening and non-balanced economic growth. Working Paper 12475, National Bureau of Economic Research.

Acemoglu, D. and Jensen, M. K. (2012). Robust comparative statics in large dynamic economies. Working Paper 18178, National Bureau of Economic Research.

Achdou, Y., Han, J., Lasry, J.-M., Lions, P.-L., and Moll, B. (2017). Income and wealth distribution in macroeconomics: A continuous-time approach. Working Paper 23732, National Bureau of Economic Research.

Agarwal, S., Liu, C., and Souleles, N. S. (2007). The reaction of consumer spending and debt to tax rebates - evidence from consumer credit data. Working Paper 13694, National Bureau of Economic Research.

Agarwal, S. and Qian, W. (2014). Consumption and debt response to unanticipated income shocks: Evidence from a natural experiment in singapore. American Economic Review, 104(12):4205-30.

Aguiar, M. A. and Bils, M. (2011). Has consumption inequality mirrored income inequality? Working Paper 16807, National Bureau of Economic Research.

Ahn, S., Kaplan, G., Moll, B., Winberry, T., and Wolf, C. (2017). When inequality matters for macro and macro matters for inequality. Working Paper 23494, National Bureau of Economic Research.

Aiyagari, S. R. (1994). Uninsured idiosyncratic risk and aggregate saving. The Quarterly Journal of Economics, 109(3):659-684.

Attanasio, O., Battistin, E., and Ichimura, H. (2007). What Really Happened to Consumption Inequality in the United States?, pages 515-543. University of Chicago Press.

Attanasio, O. and Pistaferri, L. (2014). Consumption Inequality over the Last Half Century: Some Evidence Using the New PSID Consumption Measure. American Economic Review, 104(5):122-126.

Baker, S. R. (2018). Debt and the response to household income shocks: Validation and application of linked financial account data. Journal of Political Economy, 126(4):1504-1557. 
Baker, S. R. and Yannelis, C. (2017). Income Changes and Consumption: Evidence from the 2013 Federal Government Shutdown. Review of Economic Dynamics, 23:99-124.

Baumol, W. J. (1967). Macroeconomics of unbalanced growth: The anatomy of urban crisis. American Economic Review, 57(3):415-426.

Blundell, R., Pistaferri, L., and Preston, I. (2008). Consumption inequality and partial insurance. American Economic Review, 98(5):1887-1921.

Boppart, T. (2014). Structural change and the kaldor facts in a growth model with relative price effects and non-gorman preferences. Econometrica, 82(6):2167-2196.

Browning, M. and Crossley, T. (2001). Unemployment insurance benefit levels and consumption changes. Journal of Public Economics, 80(1):1-23.

Buera, F. J. and Kaboski, J. P. (2009). The rise of the service economy. Working Paper 14822, National Bureau of Economic Research.

Carroll, C. D. (1992). The Buffer-Stock Theory of Saving: Some Macroeconomic Evidence. Brookings Papers on Economic Activity, 23(2):61-156.

Chenery, H. B. (1960). Patterns of industrial growth. The American Economic Review, 50(4):624-654.

Chimerine, L. and Incorporated, M. I. (1997). Americans in Debt: The Reality. Mastercard International Incorporated.

Clark, C. (1940). The conditions of economic progress. London : Macmillan. Includes bibliography.

Comin, D. A., Lashkari, D., and Mestieri, M. (2015). Structural change with long-run income and price effects. Working Paper 21595, National Bureau of Economic Research.

Cutler, D. M. and Katz, L. F. (1992). Rising inequality? changes in the distribution of income and consumption in the 1980s. Working Paper 3964, National Bureau of Economic Research.

Deaton, A. (1986). Demand Analysis. New-Holland, Amsterdam and New York.

Deaton, A. and Muellbauer, J. (1980). An almost ideal demand system. The American Economic Review, 70(3):312-326.

Feenstra, R. C., Inklaar, R., and Timmer, M. P. (2015). The next generation of the penn world table. American Economic Review, 105(10):3150-82.

Fisher, A. G. B. (1939). Production, primary, secondary and tertiary. The Economic Record, 15(1):24-38. 
Foellmi, R. and ZweimÃijller, J. (2008). Structural change, engel's consumption cycles and kaldor's facts of economic growth. Journal of Monetary Economics, 55(7):1317-1328.

Gelman, M., Kariv, S., Shapiro, M., Silverman, D., and Tadelis, S. (2014). Microeconomics. harnessing naturally occurring data to measure the response of spending to income. Science (New York, N.Y.), 345:212-5.

Gelman, M., Kariv, S., Shapiro, M. D., Silverman, D., and Tadelis, S. (2018). How individuals respond to a liquidity shock: Evidence from the 2013 government shutdown. Journal of Public Economics.

Gollin, D., Parente, S. L., and Rogerson, R. (2007). The food problem and the evolution of international income levels. Journal of Monetary Economics, 54(4):1230-1255.

Greene, W. (2003). Econometric Analysis, volume 89.

Gruber, J. (1997). The consumption smoothing benefits of unemployment insurance. The American Economic Review, 87(1):192-205.

Herrendorf, B., Rogerson, R., and Valentinyi, Ã. (2009). Two perspectives on preferences and structural transformation. Working Paper 15416, National Bureau of Economic Research.

Herrendorf, B., Rogerson, R., and Valentinyi, Ã. (2013). Growth and structural transformation. Working Paper 18996, National Bureau of Economic Research.

Hildenbrand, W., Mas-Colell, A., and Debreu, G. (1986). Contributions to Mathematical Economics in Honor of Gérard Debreu. North Holland.

Hsieh, C.-T. (2003). Do consumers react to anticipated income changes? evidence from the alaska permanent fund. The American Economic Review, 93(1):397-405.

Jappelli, T. (1990). Who is credit constrained in the u. s. economy? The Quarterly Journal of Economics, 105(1):219-234.

Jappelli, T., Pischke, J.-S., and Souleles, N. S. (1998). Testing for liquidity constraints in euler equations with complementary data sources. The Review of Economics and Statistics, 80(2):251-262.

Jappelli, T. and Pistaferri, L. (2010). The consumption response to income changes. Working Paper 15739, National Bureau of Economic Research.

Jappelli, T. and Pistaferri, L. (2014). Fiscal policy and mpc heterogeneity. American Economic Journal: Macroeconomics, 6(4):107-36.

Jensen, M. K. (2017). Distributional Comparative Statics. The Review of Economic Studies, 85(1):581-610. 
Johnson, D. S., Parker, J. A., and Souleles, N. S. (2004). Household expenditure and the income tax rebates of 2001. Working Paper 10784, National Bureau of Economic Research.

Kaplan, G., Moll, B., and Violante, G. L. (2016). Monetary policy according to hank. Working Paper 21897, National Bureau of Economic Research.

Kaplan, G. and Violante, G. L. (2011). A model of the consumption response to fiscal stimulus payments. Working Paper 17338, National Bureau of Economic Research.

Kaplan, G., Violante, G. L., and Weidner, J. (2014). The wealthy hand-tomouth. Working Paper 20073, National Bureau of Economic Research.

Kongsamut, P., Rebelo, S., and Xie, D. (1997). Beyond balanced growth. Working Paper 6159, National Bureau of Economic Research.

Krueger, D. and Perri, F. (2002). Does income inequality lead to consumption inequality? evidence and theory. Working Paper 9202, National Bureau of Economic Research.

Kuznets, S. (1955). Economic growth and income inequality. The American Economic Review, 45(1):1-28.

Kuznets, S. (1973). Modern economic growth: Findings and reflections. The American Economic Review, 63(3):247-258.

Maddison, A. (1980). Economic growth and structural change in advanced countries. Western Economies in Transition, pages 41-60.

Matsuyama, K. (2016). The generalized engels law in search for a new framework. Economic Theory.

Matsuyama, K. (2019). Engels law in the global economy: Demand-induced patterns of structural change, innovation, and trade. Econometrica, 87(2):497-528.

Mazzolari, F. and Ragusa, G. (2013). Spillovers from high-skill consumption to low-skill labor markets. The Review of Economics and Statistics, 95(1):7486.

Moro, A., Moslehi, S., and Tanaka, S. (2017). Does home production drive structural transformation? American Economic Journal: Macroeconomics, 9(3):116-46.

Ngai, L. R. and Pissarides, C. A. (2007). Structural change in a multisector model of growth. The American Economic Review, 97(1):429-443.

Parker, J. A. (1999). The reaction of household consumption to predictable changes in social security taxes. The American Economic Review, 89(4):959973. 
Parker, J. A., Souleles, N. S., Johnson, D. S., and McClelland, R. (2011). Consumer spending and the economic stimulus payments of 2008. Working Paper 16684, National Bureau of Economic Research.

Paxson, C. H. (1993). Consumption and income seasonality in thailand. Journal of Political Economy, 101(1):39-72.

Poterba, J. M. (1988). Are consumers forward looking? evidence from fiscal experiments. The American Economic Review, 78(2):413-418.

Shapiro, M. D. and Slemrod, J. (2001). Consumer response to tax rebates. Working Paper 8672, National Bureau of Economic Research.

Souleles, N. S. (2002). Consumer response to the reagan tax cuts. Journal of Public Economics, 85(1):99 - 120.

Stephens, Melvin, J. (2002). '3rd of tha month': Do social security recipients smooth consumption between checks? Working Paper 9135, National Bureau of Economic Research.

Stephens, Melvin, J. (2003). The consumption response to predictable changes in discretionary income: Evidence from the repayment of vehicle loans. Working Paper 9976, National Bureau of Economic Research.

Stephens, Melvin, J. and Unayama, T. (2010). The consumption response to seasonal income: Evidence from japanese public pension benefits. Working Paper 16342, National Bureau of Economic Research.

Wilcox, D. W. (1989). Social security benefits, consumption expenditure, and the life cycle hypothesis. Journal of Political Economy, 97(2):288-304.

Young, A. (2012). The african growth miracle. Working Paper 18490, National Bureau of Economic Research.

Zeldes, S. P. (1989). Consumption and liquidity constraints: An empirical investigation. Journal of Political Economy, 97(2):305-346.

ÃAzkos Valentinyi and Herrendorf, B. (2008). Measuring factor income shares at the sectoral level. Review of Economic Dynamics, 11(4):820 - 835. 


\section{Appendix A \\ Chapter 1}

\section{A.1}

\section{Additional Tables and Figures}

\begin{tabular}{|c|c|c|c|c|}
\hline & \multicolumn{4}{|c|}{ log change $1983-1985 / 2008-2010$} \\
\hline & $\mathrm{q}_{2}$ & $\mathrm{q}_{3}$ & $\mathrm{q}_{4}$ & $\mathrm{q}_{5}$ \\
\hline Consumption expenditure & 0.02 & -0.01 & 0.00 & 0.04 \\
\hline Agriculture & -0.04 & -0.06 & -0.05 & -0.08 \\
\hline Manufacturing & 0.01 & -0.04 & -0.09 & -0.06 \\
\hline Services & 0.03 & 0.01 & 0.05 & 0.11 \\
\hline Nondurable expenditures & 0.01 & 0.00 & 0.03 & 0.08 \\
\hline Agriculture & -0.04 & -0.06 & -0.05 & -0.08 \\
\hline Manufacturing & -0.05 & -0.09 & -0.10 & -0.06 \\
\hline Services & 0.04 & 0.02 & 0.06 & 0.12 \\
\hline Before-tax income & 0.03 & 0.05 & 0.14 & 0.21 \\
\hline After-tax income & 0.06 & 0.08 & 0.17 & 0.24 \\
\hline
\end{tabular}

Table A.1: Trends in Inequality: Log Change of the Ratio of each Income Quintile to Bottom Income Quintile - Consumption Expenditure, Nondurable Expenditure, Before-tax Income and After-tax income

Notes: This table depicts the log difference of the ratio of the average of each quintile of before-tax income respondents (quintile 2-quintile 5: $q_{2}-q_{5}$ ) to the average for bottom quintile respondents where the averages are taken over the pooled years indicated at the head of the table. All variables are converted into constant 1983 dollars by CPI-U - U.S. city average before averaging. Definitions of each series and sample construction are given in the data section. 


\begin{tabular}{|c|c|c|c|c|c|c|}
\hline \multirow{3}{*}{ Good category } & \multicolumn{3}{|c|}{ Consumption Expenditure } & \multicolumn{3}{|c|}{ Nondurable Expenditure } \\
\hline & \multirow{2}{*}{$\begin{array}{c}\text { CE share } \\
1980-1982\end{array}$} & \multicolumn{2}{|c|}{ First Stage Estimation } & \multirow{2}{*}{$\begin{array}{c}\text { CE share } \\
1980-1982\end{array}$} & \multicolumn{2}{|c|}{ First Stage Estimation } \\
\hline & & Elasticity & SE & & Elasticity & SE \\
\hline Housing & 23.9 & 1.03 & $(0.04)$ & 28.8 & 1.10 & $(0.04)$ \\
\hline Food at home & 14.0 & 0.34 & $(0.03)$ & 16.9 & 0.36 & $(0.03)$ \\
\hline All other tansportation & 11.1 & 0.90 & $(0.04)$ & 12.8 & 0.97 & $(0.04)$ \\
\hline Vehicle purchasing & 7.4 & 1.16 & $(0.16)$ & - & - & - \\
\hline Utilities & 5.7 & 0.50 & $(0.04)$ & 6.9 & 0.53 & $(0.04)$ \\
\hline Food away from home & 5.3 & 1.33 & $(0.07)$ & 6.4 & 1.42 & $(0.07)$ \\
\hline $\begin{array}{l}\text { Appliances, phones, computers } \\
\text { with associated services }\end{array}$ & 4.5 & 1.05 & $(0.07)$ & 0.3 & 1.50 & $(0.12)$ \\
\hline Health expenditures including insurance & 4.0 & 0.63 & $(0.07)$ & 4.7 & 0.64 & $(0.08)$ \\
\hline $\begin{array}{l}\text { Entertainment equipment and } \\
\text { subscription television }\end{array}$ & 3.3 & 1.28 & $(0.13)$ & 1.4 & 1.02 & $(0.09)$ \\
\hline Men's and women's clothing & 3.1 & 1.28 & $(0.07)$ & 3.8 & 1.37 & $(0.08)$ \\
\hline Vehicle leasing and insurance & 2.4 & 1.04 & $(0.06)$ & 2.6 & 1.11 & $(0.07)$ \\
\hline Entertainment fees, admissions, reading & 2.4 & 1.35 & $(0.06)$ & 2.5 & 1.42 & $(0.07)$ \\
\hline Cash contributions (not for alimony/support) & 2.3 & 1.39 & $(0.13)$ & 2.7 & 1.47 & $(0.14)$ \\
\hline Alcoholic beverages & 2.0 & 1.45 & $(0.12)$ & 2.4 & 1.57 & $(0.13)$ \\
\hline Shoes and other apparel & 1.8 & 1.17 & $(0.08)$ & 1.6 & 1.09 & $(0.07)$ \\
\hline Furniture purchasing & 1.6 & 1.29 & $(0.17)$ & - & - & - \\
\hline Tobacco, other smoking & 1.2 & 0.16 & $(0.10)$ & 1.4 & 0.18 & $(0.11)$ \\
\hline Education & 1.1 & 1.44 & $(0.27)$ & 1.3 & 1.53 & $(0.31)$ \\
\hline Personal care & 0.9 & 0.87 & $(0.06)$ & 1.1 & 0.92 & $(0.07)$ \\
\hline Domestic services and childcare & 0.9 & 0.80 & $(0.14)$ & 1.1 & 0.84 & $(0.15)$ \\
\hline Children's clothing (up to age 15 ) & 0.7 & 0.23 & $(0.08)$ & 0.9 & 0.24 & $(0.09)$ \\
\hline Furniture fixtures & 0.4 & 1.20 & $(0.12)$ & 0.5 & 1.27 & $(0.12)$ \\
\hline
\end{tabular}

Table A.2: Consumption Expenditure Shares and First-Stage Expenditure Elasticities of Goods Categories - 1980-1982 - Consumption Expenditure and Nondurable Expenditure

Notes: The first column presents each good's average share of total expenditure for 1980-1982 for consumption expenditure and nondurable expenditure. The remaining columns report estimates of each good's expenditure elasticity, with associated standard errors in parentheses. The regression specification sums each household's expenditure (on each good and in total) over all four interviews and instruments log total expenditure with dummy variables indicating the household's income category as well as the continuous variable of log real after-tax income. See text for details of sample construction and regression specification. It includes demographic control dummies for age, household size, and number of earners. 


\begin{tabular}{|c|c|c|c|c|c|c|}
\hline & $\begin{array}{c}\text { OLS } \\
\text { Consumption } \\
\text { expenditure }\end{array}$ & $\begin{array}{c}\text { OLS } \\
\text { Nondurable } \\
\text { expenditure }\end{array}$ & $\begin{array}{c}\text { WLS } \\
\text { Consumption } \\
\text { expenditure }\end{array}$ & $\begin{array}{c}\text { WLS } \\
\text { Nondurable } \\
\text { expenditure }\end{array}$ & $\begin{array}{l}\text { Before-tax } \\
\text { income }\end{array}$ & $\begin{array}{l}\text { After-tax } \\
\text { income }\end{array}$ \\
\hline$\delta_{q_{2},(08-10)}-\delta_{q_{2},(83-85)}$ & $\begin{array}{l}0.11 * * \\
(0.05)\end{array}$ & $\begin{array}{c}0.08 \\
(0.05)\end{array}$ & $\begin{array}{c}0.05 \\
(0.05)\end{array}$ & $\begin{array}{c}0.03 \\
(0.05)\end{array}$ & 0.03 & 0.07 \\
\hline$\delta_{q_{3},(08-10)}-\delta_{q_{3},(83-85)}$ & $\begin{array}{c}0.09 \\
(0.06)\end{array}$ & $\begin{array}{c}0.04 \\
(0.05)\end{array}$ & $\begin{array}{c}0.01 \\
(0.05)\end{array}$ & $\begin{array}{l}-0.03 \\
(0.05)\end{array}$ & 0.05 & 0.09 \\
\hline$\delta_{q_{4},(08-10)}-\delta_{q_{4},(83-85)}$ & $\begin{array}{c}0.19 * * * \\
(0.07)\end{array}$ & $\begin{array}{l}0.14 * \\
(0.07)\end{array}$ & $\begin{array}{r}0.12 * * \\
(0.05)\end{array}$ & $\begin{array}{c}0.09 \\
(0.06)\end{array}$ & 0.14 & 0.17 \\
\hline$\delta_{q_{5},(08-10)}-\delta_{q_{5},(83-85)}$ & $\begin{array}{c}0.34 * * * \\
(0.09)\end{array}$ & $\begin{array}{l}0.23 * * \\
(0.10)\end{array}$ & $\begin{array}{c}0.34 * * * * \\
(0.07)\end{array}$ & $\begin{array}{c}0.24 * * * \\
(0.07)\end{array}$ & 0.21 & 0.24 \\
\hline
\end{tabular}

Table A.3: Trends in Consumption Inequality Based on Relative Expenditure Patterns and Trends in Income Inequality - Consumption Expenditure and Nondurable Expenditure

Notes: This table reports the estimated change in consumption inequality for income quintiles versus the poorest income quintile obtained from the second-stage regressions in subsection 3.1.2. All sepecifications in columns 1-4 use the first-stage estimated expenditure elasticities reported in Table A.2 in appendix A. The estimated parameters, $\delta_{q_{k},(08-10)}-$ $\delta_{q_{k},(83-85)}, q_{k} \in\left\{q_{2}, q_{3}, q_{4}, q_{5}\right\}$, represent the relative growth in total expenditure for higherincome quintiles households relative to the lowest-income households for the period 19831985/2008-2010. See the specification in equation (8) and discussion in the text for full details. The first column implements the second stage by OLS while the second column implements weighted least squares, using the average shares for 1980-2010 as weights from NIPA-BEA. The standard errors are calculated using a bootstrap with 100 replications. 

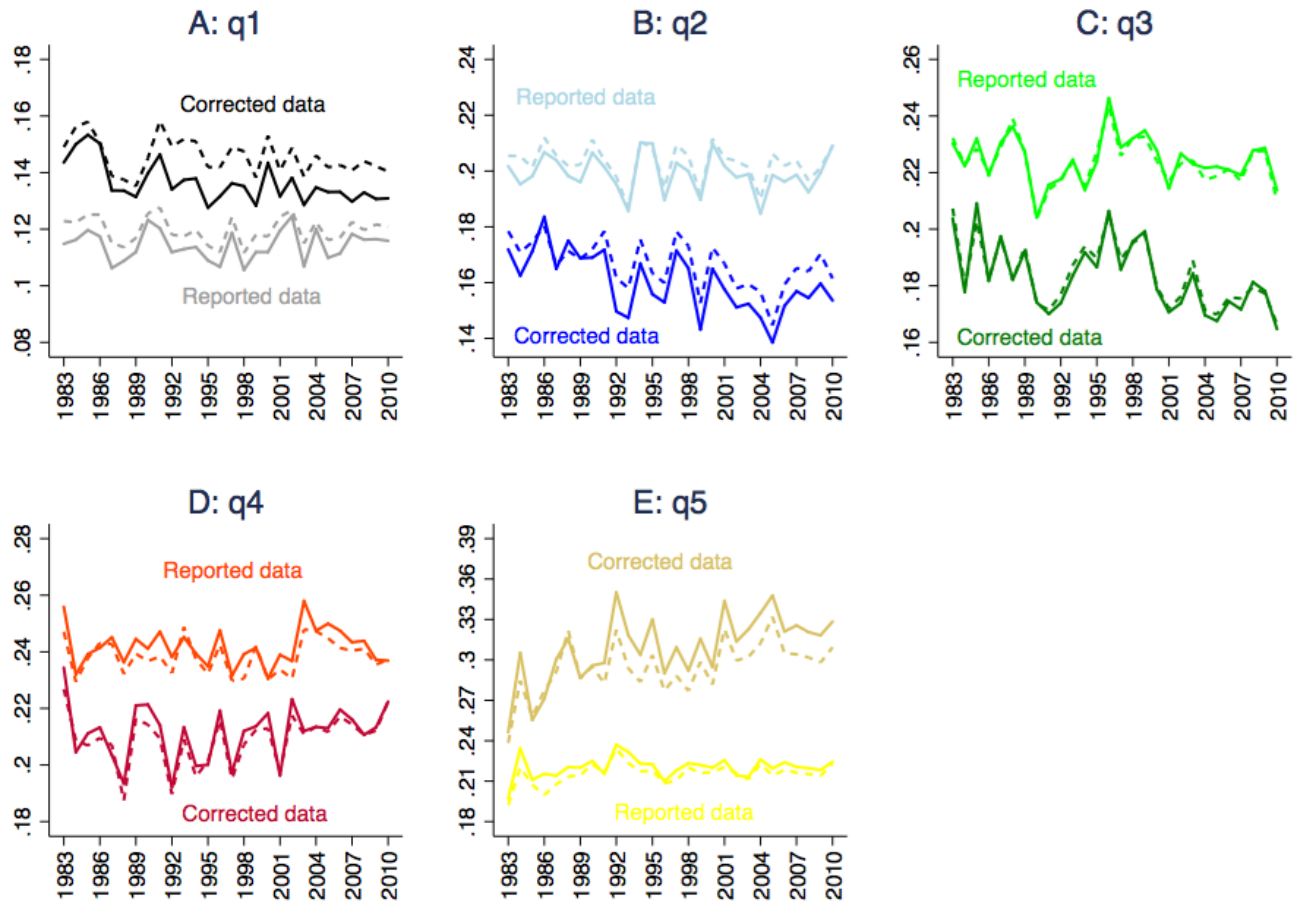

Solid line: consumption expenditure; Dashed line: nondurable expenditure

Figure A.1: Trends of the Quintiles' Expenditure Weights in Total Expenditure Computed From Reported Data in the CE Survey and Constructed From the Second-stage Regressions (Corrected Data) - Consumption Expenditure and Nondurable Expenditure Notes: This figure depicts the weights of

the average of each income quintile (quintile 1-quintile 5: $q_{1}-q_{5}$ ) with respect to total consumption expenditures from reported data in CE survey and obtained from the second-stage regressions in subsection 3.1.2. Quintiles' figures at a point in time add up to one. The weights obtained from from the second-stage regressions represent the ratio of the relative expenditure of each quintile to the sum of quintiles' relative expenditures. See the specification in equation (9) and discussion in the text for full details. Dashed lines and solid lines denote, respectively, weights of nondurable expenditures and weights of consumption expenditures. 


\begin{tabular}{ccccc}
\hline & $\begin{array}{c}\text { OLS } \\
\text { Consumption } \\
\text { expenditure }\end{array}$ & $\begin{array}{c}\text { OLS } \\
\text { Nondurable } \\
\text { expenditure }\end{array}$ & $\begin{array}{c}\text { WLS } \\
\text { Consumption } \\
\text { expenditure }\end{array}$ & $\begin{array}{c}\text { Wondurable } \\
\text { expenditure }\end{array}$ \\
\hline$\phi_{q_{2},(08-10)}-\phi_{q_{2},(83-85)}$ & $-0.11^{* *}$ & $-0.10^{*}$ & -0.03 & -0.02 \\
$\phi_{q_{3},(08-10)}-\phi_{q_{3},(83-85)}$ & $(0.05)$ & $(0.06)$ & $(0.04)$ & $(0.04)$ \\
$\phi_{q_{4},(08-10)}-\phi_{q_{4},(83-85)}$ & $-0.09^{*}$ & -0.06 & 0.02 & 0.04 \\
$\phi_{q_{5},(08-10)}-\phi_{q_{5},(83-85)}$ & $-0.05)$ & $(0.05)$ & $(0.04)$ & $(0.04)$ \\
\hline
\end{tabular}

Table A.4: Change In Relative Income-Specific Measurement Error

Notes: This table reports the change in the estimated income-specific measurement error for highest-income respondents relative to lowest-income respondents: $\phi_{q_{k},(08-10)}-\phi_{q_{k},(83-85)}$, $q_{k} \in\left\{q_{2}, q_{3}, q_{4}, q_{5}\right\}$ from equation (12) for the change over the period 1983-1985/20082010. The specification for each column is the same as in Table A.3. Standard errors are calculated using a bootstrap with 100 replications. 

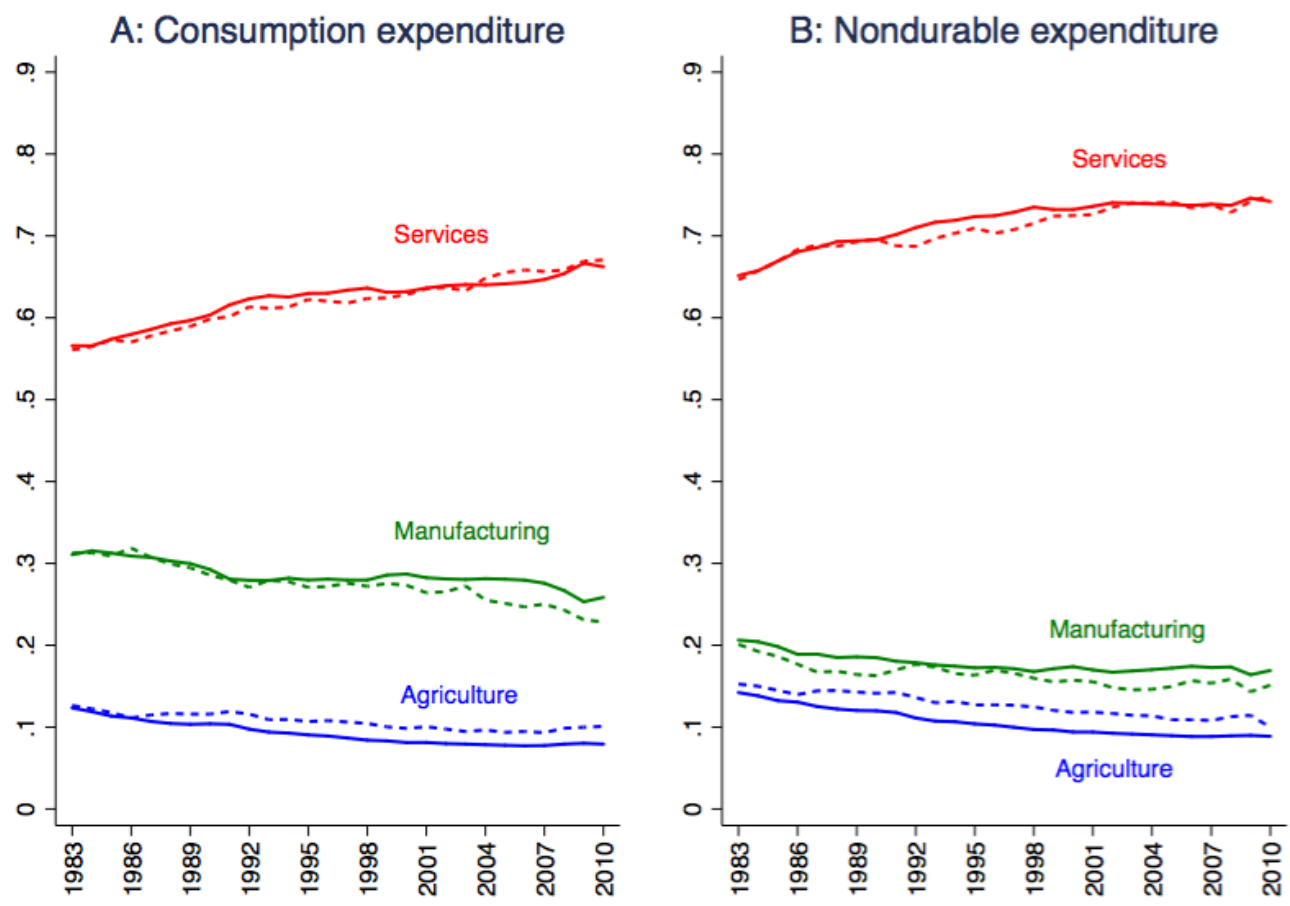

Solid line: NIPA-BEA data; Dashed line: CE-BLS data

Figure A.2: Trends in Aggregate Sectoral Shares - Consumption Expenditure and Nondurable Expenditure Notes: This figure depicts the approximation of aggregate shares of consumption expenditure and non-durable expenditure using data from CE-BLS with data from NIPA-BEA for agriculture, manufacturing and services. Solid and dashed lines denote, respectively, data from NIPA-BEA and data from CE-BLS. Sectoral shares are defined by the ratio of sectoral expenditure to total expenditure. See the data subsection 3.2.1 for further details on definitions. 


\begin{tabular}{ccc}
\hline Sector & $\begin{array}{c}\text { CE consumption category } \\
\text { (Nondurables) }\end{array}$ & CPI item \\
\hline Services & Housing & Shelter \\
Agriculture & Food at home \\
Manufactured & All other tansportation & Food at home \\
Services & Utilities & Transportation \\
Services & Food away from home & Utilities \\
Manufactured & Appliances, phones, computers & with associated services \\
Services & Health expenditures including insurance & Food away from home \\
Services & Entertainment equipment and & subscription television \\
Manufactured & Men's and women's clothing & Housekeeping supplies \\
Services & Vehicle leasing and insurance & Medical services \\
Services & Entertainment fees, admissions, reading & Tv cable \\
Services & Cash contributions (not for alimony/support) & Apparel \\
Manufactured & Alcoholic beverages & Admissions + recreational reading \\
Manufactured & Shoes and other apparel & Services \\
Manufactured & Tobacco, other smoking & Alcoholic beverages \\
Services & Education & Apparel \\
Services & Personal care & Non-durables less food, beverages and apparel \\
Services & Tuition, other school fees, and childcare \\
Manufactured & Personal care products \\
Services & Personal care services \\
& Domestic services and childcare & Apparel \\
& Children's clothing (up to age 15) & Personal care services \\
\hline
\end{tabular}

Table A.5: Association of Broad Consumption Sectors, CE Consumption Categories (Nondurables) and CPI Items

Notes: This table depicts the assignment of each nondurable consumption category in the CE-BLS and associated CPI itens (CPI-BLS) to the three broad consumption sectors that follows the sectors definitions in Herrendorf et al. (2009) Herrendorf et al. (2013). For more details on definitions of each sector see Herrendorf et al. (2009) Herrendorf et al. (2013). For details on sample construction see the data section and Aguiar and Bils (2015). 

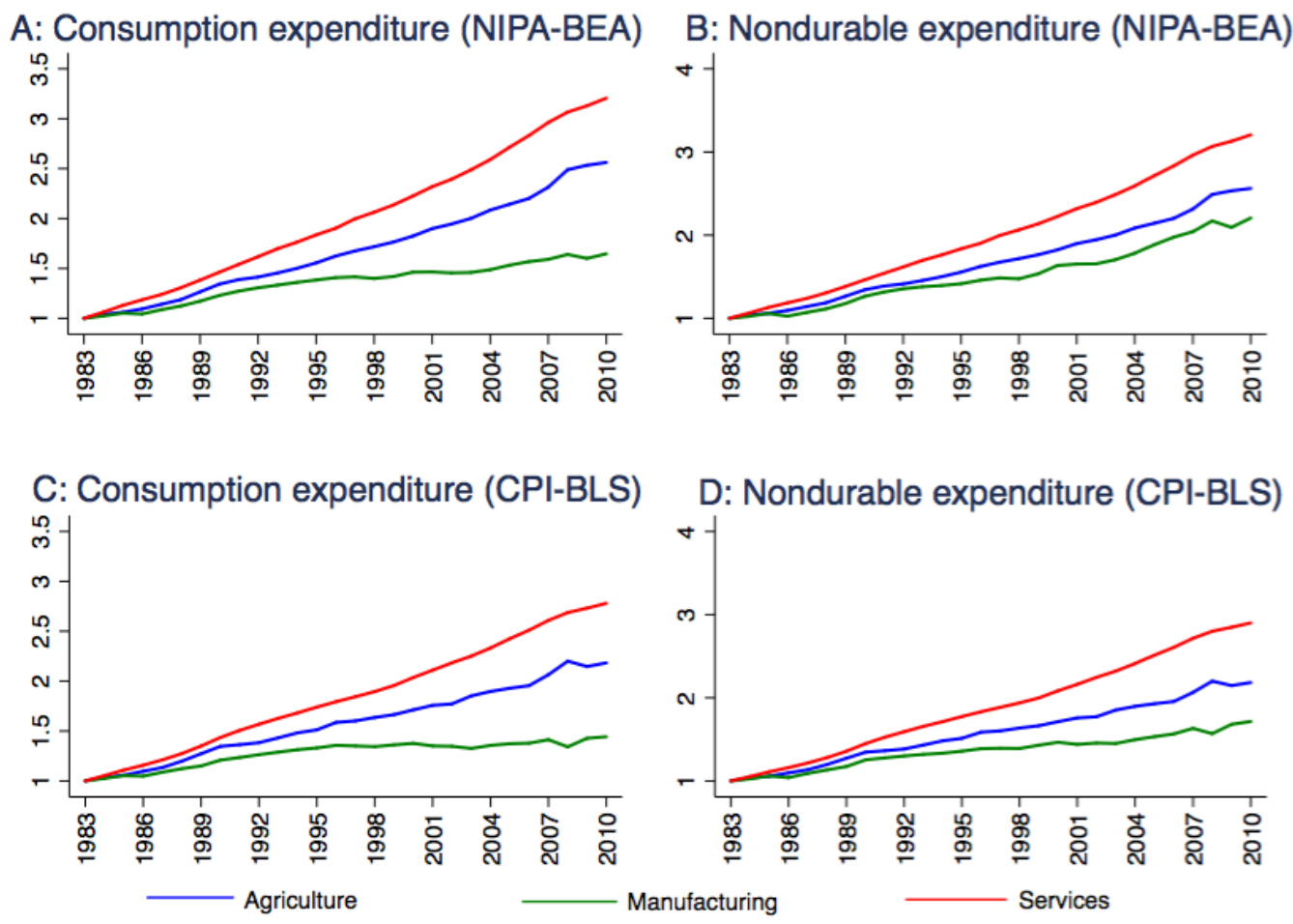

Figure A.3: Trends in Sectoral Price Indexes - Consumption Expenditure and Nondurable Expenditure Notes: This figure depicts the approximation of sectoral

price indexes considering both consumption expenditure and non-durable expenditure using data from CPI-BLS with data from NIPA-BEA for agriculture, manufacturing and services. Price indexes constructed with NIPA-BEA data are obtained by cyclical expansion procedure Herrendorf et al. (2009) (Herrendorf et al., 2013). Price indexes constructed with CPI-BLS data are obtained by weighted-average (by the expenditure share of the equivalent consumption group of CE-BLS in NIPA-BEA) of the CPI itens attributed to each consumption group that compose agriculture, manufacturing and services. Data is normalized in 1983 to 1 . See the data subsection 3.2.1 for further details on definitions. 

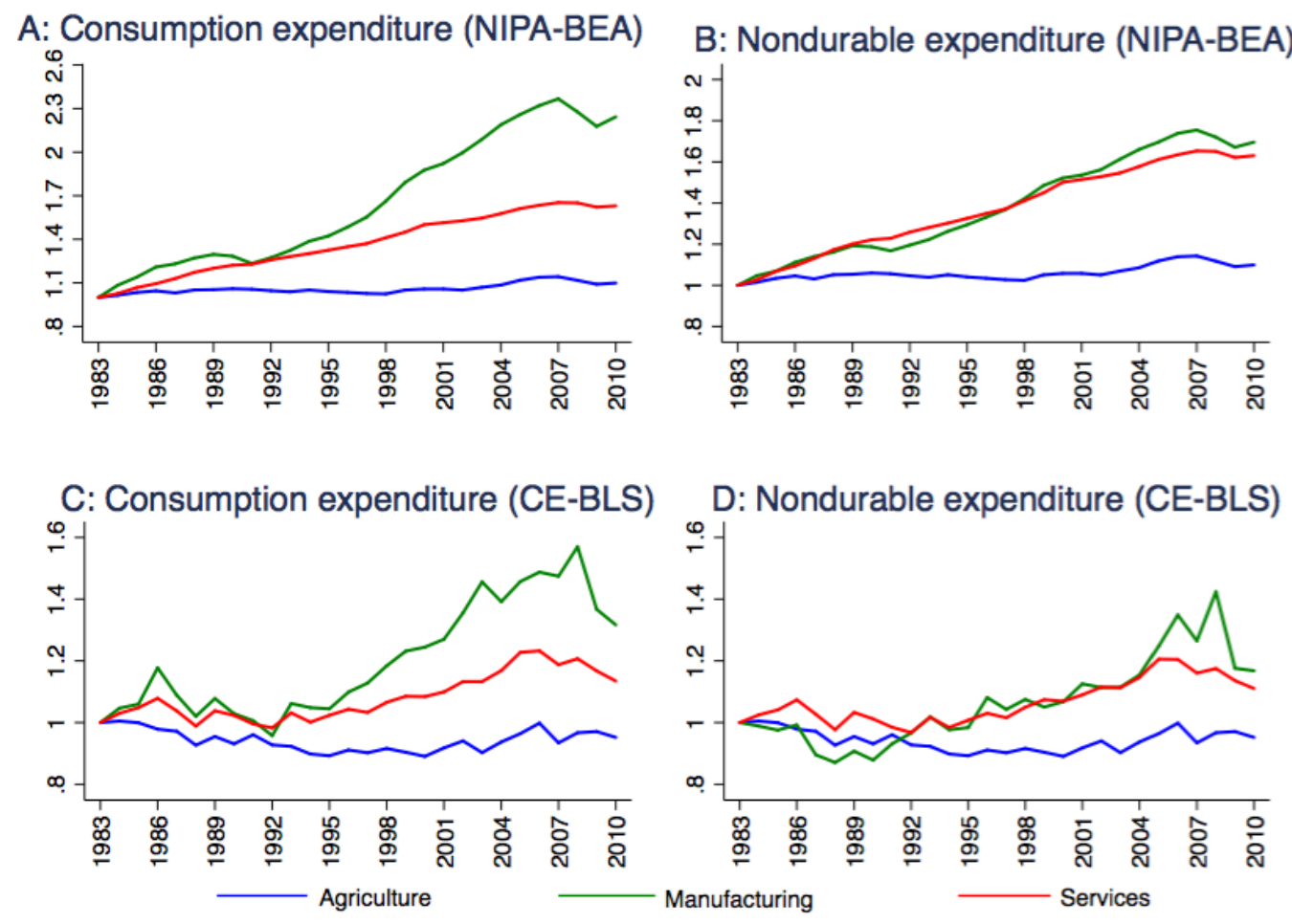

Figure A.4: Trends in Sectoral Quantity Indexes - Consumption Expenditure and Nondurable Expenditure Notes: This figure depicts the approximation of sectoral

quantity indexes considering both consumption expenditure and non-durable expenditure using data from CPI-BLS with data from NIPA-BEA for agriculture, manufacturing and services. Quantity indexes constructed with NIPA-BEA data are obtained by cyclical expansion procedure Herrendorf et al. (2009) (Herrendorf et al., 2013). Quantity indexes constructed with CPI-BLS data are obtained by deflating expenditure with the weightedaverage (by the expenditure share of the equivalent consumption group of CE-BLS in NIPABEA) of the CPI itens attributed to each consumption group that compose agriculture, manufacturing and services. Data is normalized in 1983 to 1. See the data subsection 3.2.1 for further details on definitions. 

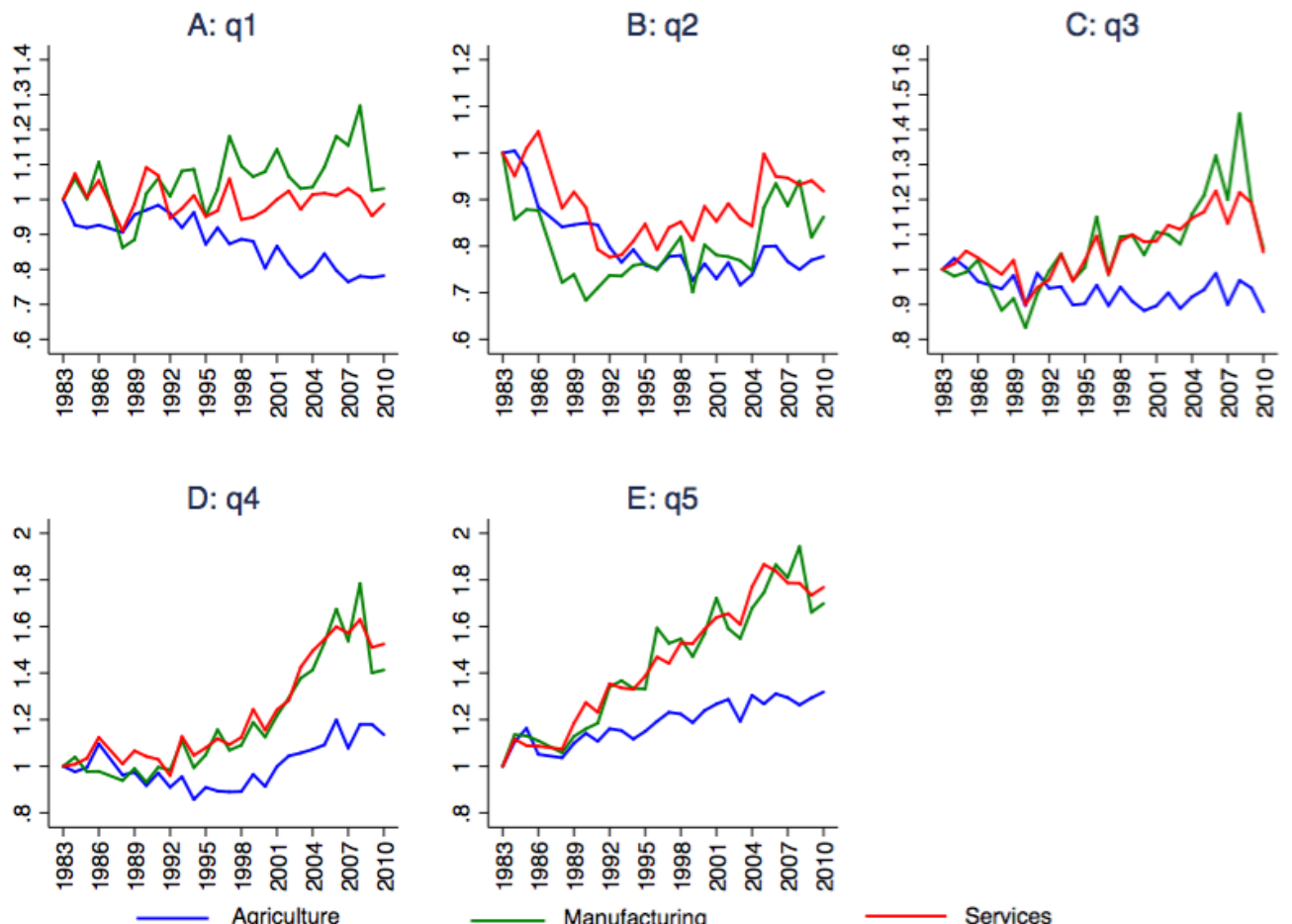

Figure A.5: Sectoral Quantity Indexes by Income Quintiles - Nondurable Expenditure

Notes: This figure depicts the sectoral quantity indexes considering nondurable expenditure using data from CPI-BLS with data from NIPA-BEA for agriculture, manufacturing and services sectors. Quantity indexes constructed with CPI-BLS data are obtained by deflating expenditure with the weighted-average (by the expenditure share of the equivalent consumption group of CE-BLS in NIPA-BEA) of the CPI itens attributed to each consumption group that compose agriculture, manufacturing and services sectors. Data is normalized in 1983 to 1. See the data subsection 3.2.1 for further details on definitions. 


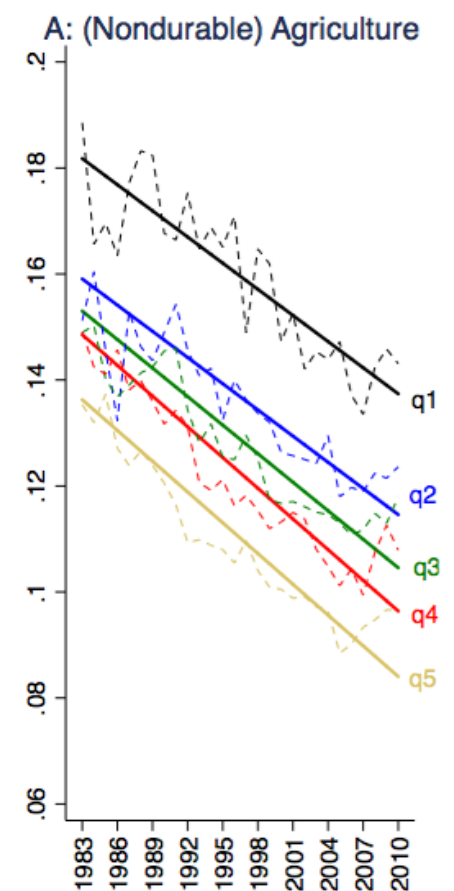

Solid line: linear trend; Dashed line: data
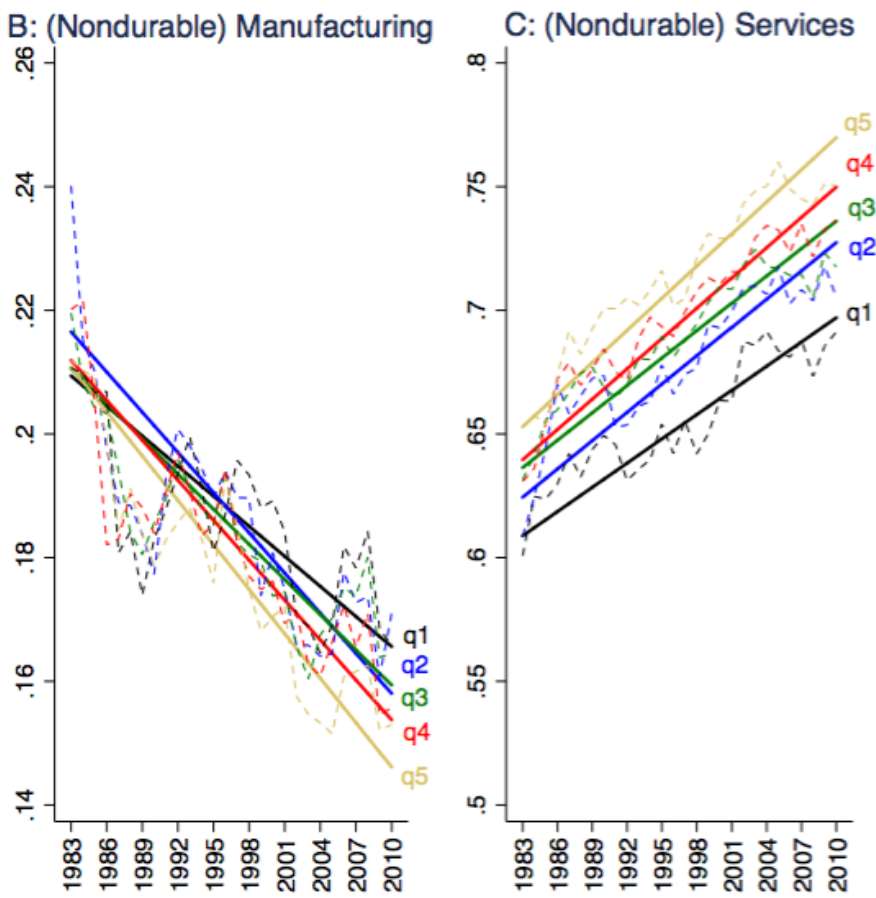

Black: q1; Blue: q2; Green: q3; Red: q4; Sand: q5

Figure A.6: Trends in Sectoral Expenditure Shares by Income Quintiles - Nondurable Expenditure Notes: This figure depicts the nondurable expenditure shares by quintiles of before-tax income respondents (quintile 1-quintile 5: $q_{1}-q_{5}$ ) in agriculture, manufacturing and services using CE-BLS data. Dashed lines and solid lines denote, respectively, data and linear trends. See the data subsection 3.2.1 for further details on definitions. 

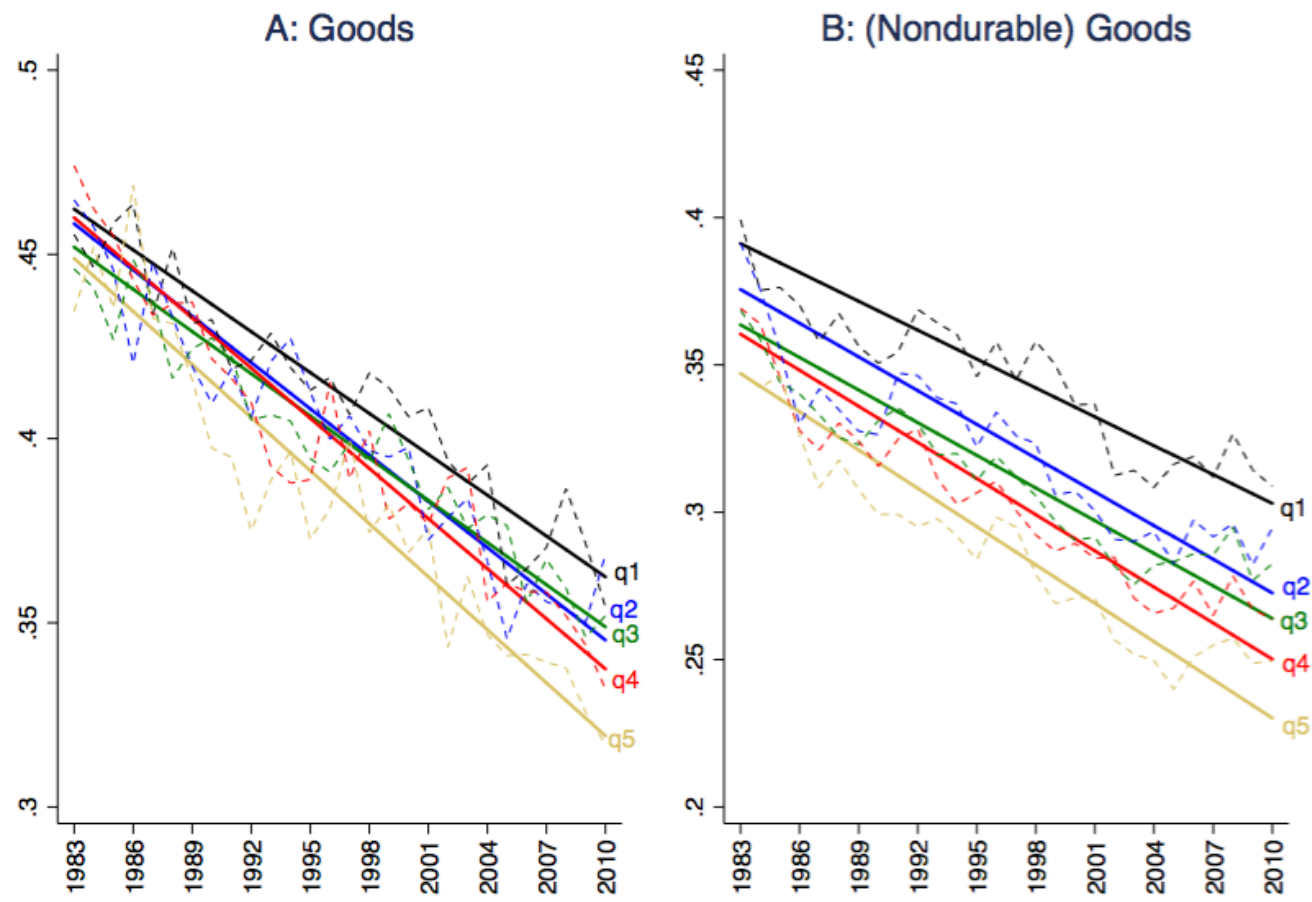

Solid line: linear trend; Dashed line: data

Black: q1; Blue: q2; Green: q3; Green: q4; Sand: q5

Figure A.7: Trends in Goods Expenditure Shares by Income Quintiles - Nondurable Expenditure Notes: This figure depicts the expenditure shares by quintiles of before-tax income respondents (quintile 1-quintile 5: $q_{1}-q_{5}$ ) in the goods sector using consumption expenditure and nondurable expenditure from the CE-BLS data. Dashed lines and solid lines denote, respectively, data and linear trends. See the data subsection 3.2.1 for further details on definitions. 


\begin{tabular}{|c|c|c|c|c|c|c|}
\hline & \multicolumn{6}{|c|}{ Nondurable expenditure } \\
\hline & $\mathrm{q}_{1}$ & $\mathrm{q}_{2}$ & $\mathrm{q}_{3}$ & $\mathrm{q}_{4}$ & $\mathrm{q}_{5}$ & Aggregate \\
\hline $\bar{c}_{a}$ & $\begin{array}{l}-229.69^{*} \\
(123.73)\end{array}$ & $\begin{array}{c}-280.66 * * * \\
(68.75)\end{array}$ & $\begin{array}{l}-643.86^{* * * *} \\
(50.30)\end{array}$ & $\begin{array}{l}-387.43^{* * * *} \\
(83.19)\end{array}$ & $\begin{array}{l}-311.53 * * * \\
(45.47)\end{array}$ & $\begin{array}{c}-769.83^{* * * *} \\
(49.30)\end{array}$ \\
\hline $\bar{c}_{s}$ & $\begin{array}{l}-1,446.39 * * * \\
\quad(113.64)\end{array}$ & $\begin{array}{l}-1,945.05^{* * * *} \\
\quad(267.78)\end{array}$ & $\begin{array}{l}-431.49 \\
(909.57)\end{array}$ & $\begin{array}{l}9,831.60^{* *} \\
(3,922.46)\end{array}$ & $\begin{array}{c}15,611.49 * * * \\
(5,802.472)\end{array}$ & $\begin{array}{c}30,734.87 \\
(-)\end{array}$ \\
\hline$\varphi_{a}$ & $\begin{array}{c}0.21 * * * \\
(0.03)\end{array}$ & $\begin{array}{c}0.32 * * * \\
(0.03)\end{array}$ & $\begin{array}{c}0.05^{* * *} \\
(0.01)\end{array}$ & $\begin{array}{c}0.03^{* * *} \\
(0.01)\end{array}$ & $\begin{array}{l}0.02 * * * \\
(0.005)\end{array}$ & $\begin{array}{c}0.003 * * * \\
(0.001)\end{array}$ \\
\hline$\varphi_{m}$ & $\begin{array}{c}0.33^{* * *} \\
(0.03)\end{array}$ & $\begin{array}{c}0.16^{* * *} \\
(0.02)\end{array}$ & $\begin{array}{c}0.22 * * * \\
(0.05)\end{array}$ & $\begin{array}{l}0.07 * * * \\
(0.02)\end{array}$ & $\begin{array}{c}0.05^{* * *} \\
(0.01)\end{array}$ & $\begin{array}{l}0.027 * * * \\
(0.004)\end{array}$ \\
\hline$\varphi_{s}$ & $\begin{array}{c}0.46^{* * *} \\
(0.03)\end{array}$ & $\begin{array}{c}0.52^{* * * *} \\
(0.04)\end{array}$ & $\begin{array}{c}0.73^{* * * *} \\
(0.06)\end{array}$ & $\begin{array}{c}0.90^{* * *} \\
(0.02)\end{array}$ & $\begin{array}{c}0.93 * * * \\
(0.02)\end{array}$ & $\begin{array}{c}0.97 * * * \\
(0.001)\end{array}$ \\
\hline$\chi^{2}\left(\bar{c}_{a}=0, \bar{c}_{s}=0\right)$ & $164.85^{* * *}$ & $58.38^{* * *}$ & $171.71 * * *$ & $36.26^{* * *}$ & $79.21 * * *$ & $243.88^{* * *}$ \\
\hline$\chi^{2}\left(\theta_{q_{k}}=\theta_{A g g}\right)$ & $3,100,000^{* * *}$ & $480,000.00$ & $170,000^{* * *}$ & $2,598.79 * * *$ & $3,326.00^{* * *}$ & - \\
\hline$L R\left(\theta_{\text {Agg }}\right.$ nested in $\left.\left\{\theta_{q_{k}}\right\}_{k=1}^{5}\right)$ & - & - & - & - & - & $1,515.16^{* * *}$ \\
\hline$R M S E_{a}$ & 0.010 & 0.009 & 0.007 & 0.009 & 0.003 & 0.006 \\
\hline$R M S E_{m}$ & 0.009 & 0.013 & 0.015 & 0.011 & 0.010 & 0.017 \\
\hline$R M S E_{s}$ & 0.014 & 0.020 & 0.021 & 0.021 & 0.012 & 0.021 \\
\hline
\end{tabular}

Table A.6: Sectoral Share Estimation Results for the Aggregate and Income Quintiles $\left(q_{1}-q_{5}\right)$-Nondurable Expenditure

Notes: $\chi^{2}\left(\bar{c}_{a}=0, \bar{c}_{s}=0\right)$ is the Wald Test Statistics for the hypothesis that $\bar{c}_{a}$ and $\bar{c}_{s}$ are jointly zero. $\chi^{2}\left(\theta_{q_{k}}=\theta_{\text {agg }}\right)$ is the Wald Test Statistics for the hypothesis that each quintiles' coefficient estimates are jointly equal to the aggregate counterpart. $\operatorname{LR}\left(\theta_{\text {agg }}\right.$ nested in $\left.\left\{\theta_{q_{k}}\right\}_{k=1}^{5}\right)$ is the Likelihood-ratio test for the hypothesis that all coefficients of the aggregate model do not vary between income quintiles $q_{1}-q_{5} . R M S E_{j}$ is the root mean squared error for j-sector's share equation. Robust standard errors in parentheses. The calculation of standard errors of the constrained parameters is based on the delta method. *** Significant at the 1 percent level, ${ }^{* *}$ Significant at the 5 percent level and $*$ Significant at the 10 percent level. 

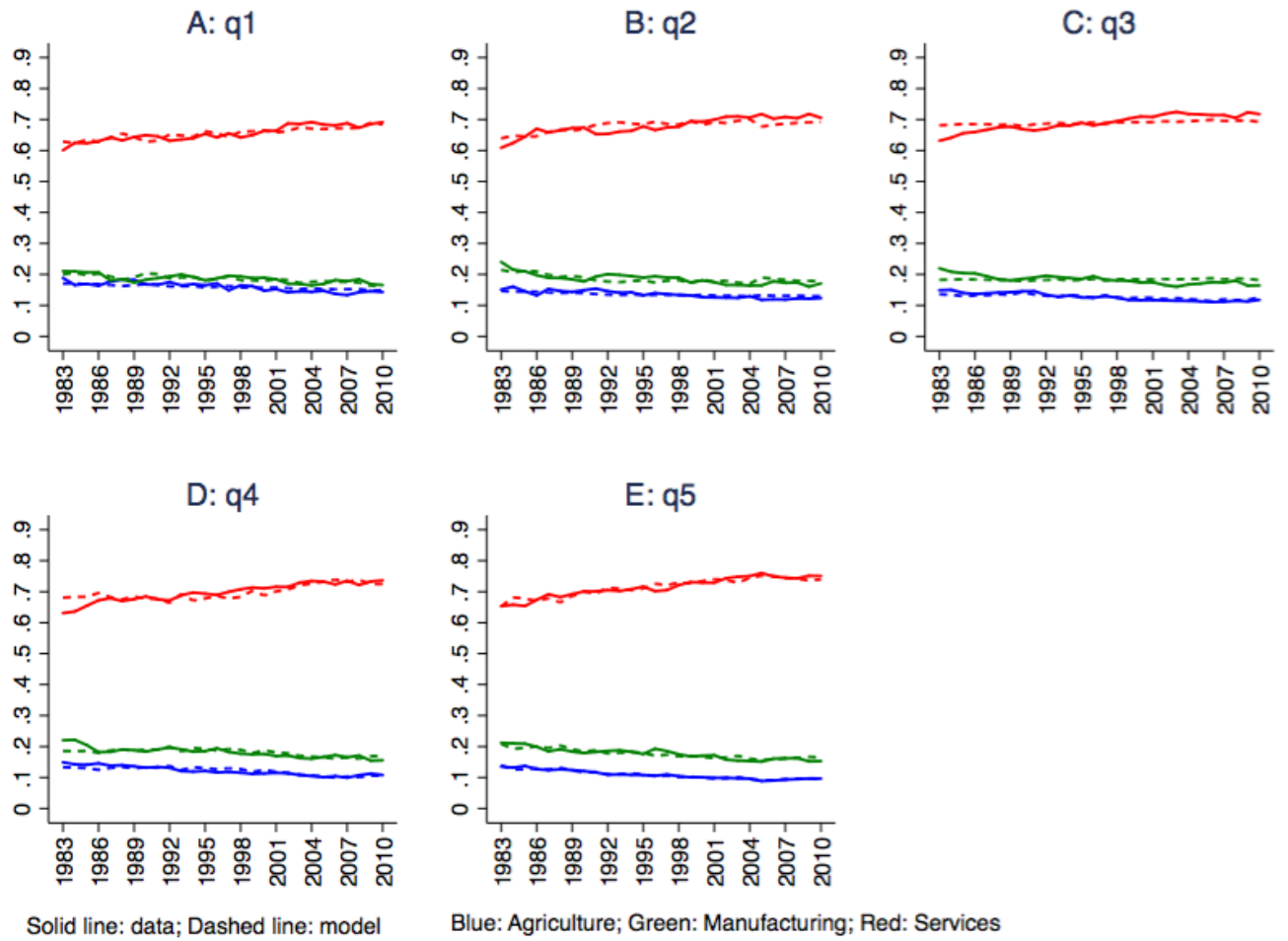

Figure A.8: Fit of the Model: Expenditure Shares by Income Quintiles - Nondurable Expenditure Notes: This figure depicts the fit of the model for sectoral nondurable expenditure shares by income quintiles (Graphs A-E, respectively, for quintile 1-quintile 5: $q_{1}-q_{5}$ ) in agriculture (blue), manufacturing (green) and services (red) as denoted by equation (3) using CE-BLS data for consumption expenditure. Solid lines and dashed lines denote, respectively, data, model prediction. See the data subsection for further details on definitions and the estimation subsection for the estimation procedure. 


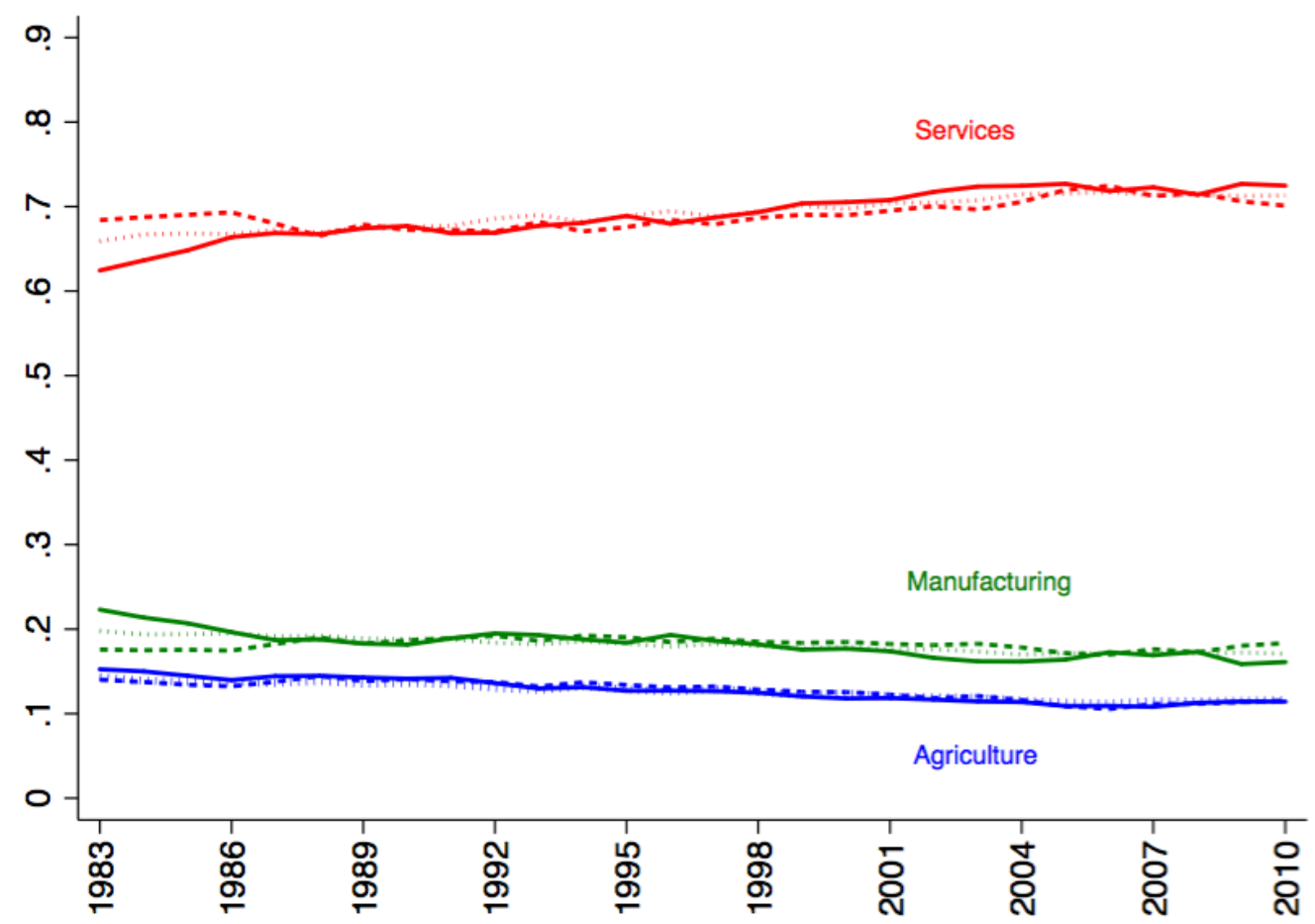

Solid line: data; Dashed line: model; Dotted line: model - weighted-average aggregate

Figure A.9: Fit of the Model: Expenditure Shares on Aggregate Data and Weighted-average Aggregate Expenditure Shares

-Nondurable Expenditure Notes: This figure depicts the fit of the model for nondurable

expenditure shares on aggregate data and the weighted-average (by expenditure weights of quintile 1-quintile 5: $q_{1}-q_{5}$ ) aggregate expenditure shares in agriculture (blue), manufacturing (green) and services (red) using CE-BLS data for consumption expenditure. Solid lines, dashed lines and dotted lines denote, respectively, data, model prediction on aggregate data and model prediction for the weighted-average aggregate expenditure shares. See the data subsection for further details on definitions and the estimation subsection for the estimation procedure. 

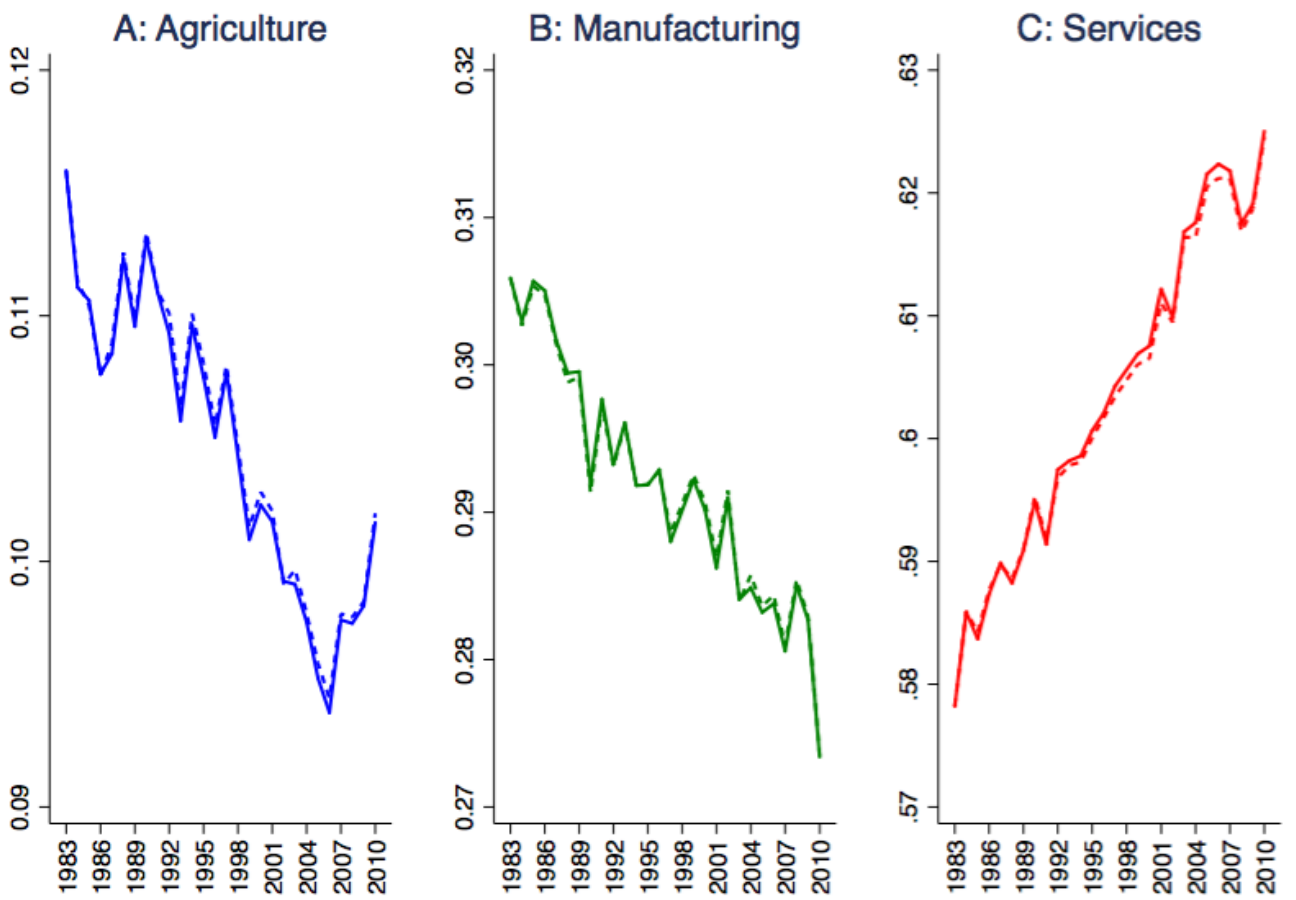

Solid line: model; Dashed line: model - counterfactual

Figure A.10: Counterfactual experiment: No Increase in Consumption Inequality - Reported Data Notes: This figure depicts the weighted-average sectoral expenditure share (model) in agriculture (Graph A), manufacturing (Graph B) and services (Graph C) and their counterfactuals in which all market prices and quintiles' expenditures evolve as observed in the data, but quintiles' weights are kept constant at their 1983 levels using CE-BLS reported data. See equations (10) and (14), respectively, for the formal definitions of the weighted-average sectoral expenditure shares and their counterfactuals so that consumption inequality is held constant at the 1983 pattern. 


\begin{tabular}{|c|c|c|c|c|}
\hline \multirow{2}{*}{$\begin{array}{l}\Delta \text { Percent of the weighted-average aggregate expenditure share } \\
\text { (Counterfactual experiment relative to the model prediction) }\end{array}$} & \multicolumn{2}{|c|}{$\begin{array}{l}\text { Consumption expenditure } \\
\text { (CE survey) }\end{array}$} & \multicolumn{2}{|c|}{$\begin{array}{c}\text { Nondurable expenditure } \\
\text { (CE survey) }\end{array}$} \\
\hline & Reported data & Corrected data & Reported data & Corrected data \\
\hline$\varsigma_{a,(08-10)}$ & 0.8 & 10.8 & 5.4 & 6.2 \\
\hline$\varsigma_{m,(08-10)}$ & 0.1 & -2.3 & 0.5 & 2.5 \\
\hline$\varsigma_{S,(08-10)}$ & 0.3 & 1.8 & 3.0 & 4.4 \\
\hline
\end{tabular}

Table A.7: Percent Change of the Variation of the Weighted-average Aggregate Expenditure Shares Attributed to the Correlation With the Increase of Consumption Inequality - 2008-2010/1983-1985

Notes: This table reports the percent change of the variation of the weighted-average aggregate sectoral share that is attributed to the correlation with the increase of consumption inequality, $\varsigma_{i,(08-10)}$, between 2008-2010/1983-1985, where $i=\{a, m, s\}$, using CE-BLS reported and corrected data. The effect is in percent change, which means it is relative to the size of the predicted change of the weighted-average aggregate sectoral shares. See equation (15) for the formal definition for corrected data. For reported data, the formal definition is analogous to equation (15) using reported quintiles' expenditures weights, $\triangle \omega_{\mathcal{K}^{\prime \prime}, t_{1}}$, instead of the corrected ones, $\triangle \hat{\omega}_{\mathcal{K}^{\prime \prime}, t_{1}}$. 


\section{A. 2}

\section{Aggregation of Demand Functions}

We follow the Online Appendix A in Herrendorf et al. (2009) Herrendorf et al. (2013) which show that there is aggregation for general case of the StoneGeary preferences, which encapsulates our model's preferences. We use a trivial decomposition to show that a stand-in household for an income quintile $q_{k}$, such that $q_{k} \in\left\{q_{1}, q_{2}, q_{3}, q_{4}, q_{5}\right\}$, is consistent with a stand-in household of the aggregate economy.

Consider $H$ households indexed by $h=1, \ldots, H$. Each household $h$ in quintile $q_{k}$ solves the following intratemporal problem:

$$
\begin{array}{cc}
\max _{\left\{c_{q_{k}, a, t}^{h}, c_{q_{k}, m, t}^{h}, c_{q_{k}, s, t}^{h}\right\}} & \sum_{i=a, m, s} \varphi_{q_{k}, i} \log \left(c_{q_{k}, i, t}^{h}+\bar{c}_{q_{k}, i}\right) \\
\sum_{i=a, m, s} p_{i, t} c_{q_{k}, i, t}^{h} & \leq
\end{array}
$$

where $e_{q_{k}, t}^{h}=p_{t} c_{q_{k}, t}^{h}-\sum_{i=a, m, s} p_{i, t} \bar{c}_{q_{k}, i}$ denotes total expenditure of household $h$ in quintile $q_{k}$, such that $c_{q_{k}, t}^{h}$ is the consumption goods composite of household $h$ in quintile $q_{k}$ and $p_{t}$ is the price of the consumption good composite.

The first order conditions yields the following marshallian demands:

$$
c_{q_{k}, i, t}^{h}=\frac{\varphi_{q_{k}, i}}{p_{i, t}}\left(e_{q_{k}, t}^{h}+\sum_{g=a, m, s} p_{g, t} \bar{c}_{q_{k}, g}\right)-\bar{c}_{q_{k}, i},
$$

where we refer to $g=\{a, m, s\}$ here with a slight abuse of terminology.

Note from Marshallian demand of services that the solution of each household problem is interior if parameters and the income distribution are such that household expenditure of the poorest household, exceeds a minimum nonnegative level.That is, for all $h \in\{1, \ldots, H\}$ household expenditure exceed a minimum level. Since households are ordered by income quintiles, we naturally refer to the bottom quintile:

$$
\begin{aligned}
p_{s, t} \bar{c}_{q_{1}, s} & <\varphi_{q^{*}, s}\left(\min _{h \in H_{q_{1}}} e_{q_{1}, t}^{h}+\sum_{i=a, m, s} p_{i, t} \bar{c}_{q_{1}, i}\right) \\
\min _{h \in H_{q_{1}}} e_{q_{1}, t}^{h} & >\left(p_{a, t} \bar{c}_{q_{1}, a}\right)+\left(\frac{1-\varphi_{q_{1}, s}}{\varphi_{q_{1}, s}}\right)\left(p_{s, t} \bar{c}_{q_{1}, s}\right) \\
e_{q_{1}, t}^{h} & >\left[p_{a, t} \max \left(-\bar{c}_{q_{1}, a}, 0\right)\right]+\left(\frac{1-\varphi_{q_{1}, s}}{\varphi_{q_{1}, s}}\right)\left[p_{s, t} \max \left(-\bar{c}_{q_{1}, s}, 0\right)\right]
\end{aligned}
$$


where we restrict $\bar{c}_{q_{k}, m}=0$ for all $q_{k}$ and $e_{q_{5}, t}^{h}>e_{q_{4}, t}^{h}>e_{q_{3}, t}^{h}>e_{q_{2}, t}^{h}>e_{q_{1}, t}^{h}>0$ for all household $h$ in quintile $q_{k}$.

Some simple algebra yields the expenditure of the sectoral commodity good $i$ so that it makes explicit the linear demand (expenditure) system:

$$
\begin{aligned}
& c_{q_{k}, i, t}^{h}=\left[\left(\frac{\varphi_{q_{k}, i}}{p_{i, t}}\right) \sum_{g=a, m, s} p_{g, t} \bar{c}_{q_{k}, g}-\bar{c}_{q_{k}, i}\right]+\left(\frac{\varphi_{q_{k}, i}}{p_{i, t}}\right) e_{q_{k}, t}^{h} . \\
& e_{q_{k}, i, t}^{h} \equiv p_{i, t} c_{q_{k}, i, t}^{h}=\left(\varphi_{q_{k}, i} \sum_{g=a, m, s} p_{g, t} \bar{c}_{q_{k}, g}-p_{i, t} \bar{c}_{q_{k}, i}\right)+\varphi_{q_{k}, i} e_{q_{k}, t}^{h} .
\end{aligned}
$$

Therefore, since there is a finite number of commodities and households and Stone-Geary preferences feature linear Engel curves ${ }^{1}$, then our specific Stone-Geary preferences can be aggregated and represented by those of a representative household in the lines we show below - Gorman's Aggregation Theorem (Gorman, 1959).

Adding up over all households, we obtain:

$$
e_{q_{k}, i, t}=\left[\varphi_{q_{k}, i} \sum_{g=a, m, s} p_{g, t}\left(H_{q_{k}} \bar{c}_{q_{k}, g}\right)-p_{i, t}\left(H_{q_{k}} \bar{c}_{q_{k}, i}\right)\right]+\varphi_{q_{k}, i} e_{q_{k}, t},
$$

where $H_{q_{k}} \equiv \sum_{h=1}^{H_{q_{k}}} h$ stands for all households in quintile $q_{k}, e_{q_{k}, i, t} \equiv \sum_{h=1}^{H_{q_{k}}} e_{q_{k}, i, t}^{h}$ and $e_{q_{k}, t} \equiv \sum_{i=a, m, s} \sum_{h=1}^{H_{q_{k}}} e_{q_{k}, i, t}^{h}$.

If the stand-in household of quintile $q_{k}$ solves:

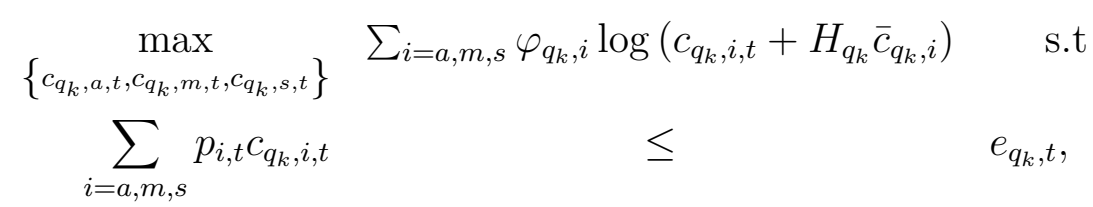

then its choices satisfy $c_{q_{k}, i, t} \equiv \sum_{h=1}^{H_{q_{k}}} c_{q_{k}, i, t}^{h}$, which means there is aggregation within quintile $q_{k}$.

Analogously for the stand-in household of the aggregate economy, consider each household $h=1, \ldots, H$ solves:

${ }^{1}$ That is, it can also be represented by an indirect utility form, $v\left(\boldsymbol{p}_{t}, e_{t}\right)=\sum_{H} a^{h}\left(\boldsymbol{p}_{t}\right)+$ $b\left(\boldsymbol{p}_{t}\right) e_{t}^{h}$, where $\boldsymbol{p}_{t}=\left(p_{1}, \ldots, p_{I}\right)$ and $e_{t} \equiv \sum_{H} e_{t}^{h}$. In our case, $v^{h}\left(\boldsymbol{p}_{t}, e_{t}^{h}\right)=e_{t}^{h}$ (see footnote 11) and then $v\left(\boldsymbol{p}_{t}, e_{t}\right)=e_{t}$. 


$$
\begin{array}{cc}
\max _{\left\{c_{a, t}^{h}, c_{m, t}^{h}, c_{s, t}^{h}\right\}} & \sum_{i=a, m, s} \varphi_{i} \log \left(c_{i, t}^{h}+\bar{c}_{i}\right) \\
\sum_{i=a, m, s} p_{i, t} c_{i, t}^{h} & \leq
\end{array}
$$

where $e_{t}^{h}=p_{t} c_{t}^{h}-\sum_{i=a, m, s} p_{i, t} \bar{c}_{i}$ denotes total expenditure of household $h$, such that $c_{t}^{h}$ is the consumption goods composite of household $h$.

Then again from the first order conditions we have the following Marshallian demands:

$$
c_{i, t}^{h}=\frac{\varphi_{i}}{p_{i, t}}\left(e_{t}^{h}+\sum_{g=a, m, s} p_{g, t} \bar{c}_{g}\right)-\bar{c}_{i} .
$$

Equivalently to the previous case for households $h$ ordered by income quintile $q_{k}$, we have that for all $h \in\{1, \ldots, H\}$, household expenditure must exceed a minimum level for the solution of each household problem to be interior:

$$
e_{t}^{h}>\left[p_{a, t} \max \left(-\bar{c}_{a}, 0\right)\right]+\left(\frac{1-\varphi_{s}}{\varphi_{s}}\right)\left[p_{s, t} \max \left(-\bar{c}_{s}, 0\right)\right]
$$

where we restrict $\bar{c}_{m}=0$ and $e_{t}^{h}>0$ for all household $h$.

Again some simple algebra yields our linear demand system:

$$
e_{i, t}^{h} \equiv p_{i, t} c_{i, t}^{h}=\left(\varphi_{i} \sum_{g=a, m, s} p_{g, t} \bar{c}_{g}-p_{i, t} \bar{c}_{i}\right)+\varphi_{i} e_{t}^{h} .
$$

Adding up over all households, we obtain:

$$
e_{i, t}=\left[\varphi_{i} \sum_{g=a, m, s} p_{g, t}\left(H \bar{c}_{g}\right)-p_{i, t}\left(H \bar{c}_{i}\right)\right]+\varphi_{i} e_{t},
$$

where $e_{i, t} \equiv \sum_{h=1}^{H} e_{i, t}^{h}=\sum_{k=1}^{5} \sum_{h=1}^{H_{q_{k}}} e_{q_{k}, i, t}^{h}, H \equiv \sum_{h=1}^{H} h=\sum_{k=1}^{5} \sum_{h=1}^{H_{q_{k}}} h$, $e_{t} \equiv \sum_{i=a, m, s} \sum_{h=1}^{H} e_{i, t}^{h}=\sum_{i=a, m, s} \sum_{k=1}^{5} \sum_{h=1}^{H_{q_{k}}} e_{q_{k}, i, t}^{h}$ and $H \bar{c}_{i} \equiv \sum_{k=1}^{5} H_{q_{k}} \bar{c}_{q_{k}, i}$.

Thus, if the stand-in household solves:

$$
\begin{array}{cc}
\max _{\left\{c_{a, t}, c_{m, t}, c_{s, t}\right\}} & \sum_{i=a, m, s} \varphi_{i} \log \left(c_{i, t}+H \bar{c}_{i}\right) \\
\sum_{i=a, m, s} p_{i, t} c_{i, t} & \leq
\end{array}
$$

then its choices satisfy $c_{i, t} \equiv \sum_{h=1}^{H} c_{i, t}^{h}=\sum_{k=1}^{5} \sum_{h=1}^{H_{q_{k}}} c_{q_{k}, i, t}^{h}$, which means there is aggregation in the economy as a whole. 
Therefore, aggregation within quintile $q_{k}$ is consistent with aggregation for the economy as a whole. 


\section{A.3}

\section{Linear Expenditure Systems of the Extensive and Intensive Margins of Consumption Expenditure}

Consider the structural linear expenditure system we use to estimate the intensive margin of consumption. Recall the intratemporal problem (1) - (2) of the stand-in household. Now considering the true total expenditure for all $h \in\{1, \ldots, H\}$, the first order conditions yields the following true marshallian demands:

$$
\left(c_{i, t}^{h}\right)^{*}=\frac{\varphi_{i}}{p_{i, t}}\left[\left(e_{t}^{h}\right)^{*}+\sum_{g=a, m, s} p_{g, t} \bar{c}_{g}\right]-\bar{c}_{i},
$$

where again we refer to $g=\{a, m, s\}$ here with a slight abuse of terminology. Moreover, remind that each sector (agriculture, manufacturing and services) aggregates consumption categories as in dataset description subsection.

Some simple algebra yields the true expenditure of the sectoral commodity good $i$ so that it makes explicit the linear demand system:

$$
\begin{aligned}
\left(e_{i, t}^{h}\right)^{*} \equiv p_{i, t}\left(c_{i, t}^{h}\right)^{*} & =\left(\varphi_{i} \sum_{g=a, m, s} p_{g, t} \bar{c}_{g}-p_{i, t} \bar{c}_{i}\right)+\varphi_{i}\left(e_{t}^{h}\right)^{*} \\
& =\gamma_{0, i, t}+\gamma_{1, i}\left(e_{t}^{h}\right)^{*},
\end{aligned}
$$

where $\gamma_{0, i, t} \equiv \varphi_{i} \sum_{g=a, m, s} p_{g, t} \bar{c}_{g}-p_{i, t} \bar{c}_{i}$ and $\gamma_{1, i} \equiv \varphi_{i}$.

We then take a first-order expansion in the true expenditure around the true mean expenditure and following some manipulation in the right-hand side we have

$$
\begin{aligned}
\ln \left(e_{i, t}^{h}\right)^{*}-\ln \bar{e}_{i, t}^{*} & =-\left(\frac{\gamma_{1, i} \bar{e}_{t}^{*} \ln \bar{e}_{t}^{*}}{\gamma_{0, i, t}+\gamma_{1, i} \bar{e}_{t}^{*}}\right)+\left(\frac{\gamma_{1, i} \bar{e}_{t}^{*}}{\gamma_{0, i, t}+\gamma_{1, i} \bar{e}_{t}^{*}}\right) \ln \left(e_{t}^{h}\right)^{*} \\
& =\alpha_{i, t}^{*}+\beta_{i}\left(e_{t}^{h}\right)^{*}
\end{aligned}
$$

where $\alpha_{i, t}^{*} \equiv-\left(\frac{\gamma_{1, i} \bar{e}_{t}^{*} \ln \bar{e}_{t}^{*}}{\gamma_{0, i, t}+\gamma_{1, i} \bar{e}_{t}^{*}}\right), \beta_{i} \equiv \frac{\gamma_{1, i} \bar{e}_{t}^{*}}{\gamma_{0, i, t}+\gamma_{1, i} \bar{e}_{t}^{*}}$ and $\bar{e}_{i, t}^{*}$ is the mean per capita expenditure of sectoral commodity good $i=\{a, m, s\}$ (that aggregates consumption categories) in year $t$ across all households and $\beta_{i}$ is assumed to be stable over time. Note that it applies to any sectoral commodity good $i$, but also to any consumption category $j, j=1, \ldots, 22$, that comprise the consumption sectors. Thus, we can represent the reduced-form log-linear Engel curves approximation in equation (6) used to estimate the extensive margin of consumption expenditure as a function of prices and parameters from our 
structural linear expenditure system that we use to estimate the intensive margin of consumption. 


\section{Appendix B \\ Chapter 2}

\section{B.1}

\section{Additional Tables and Figures}

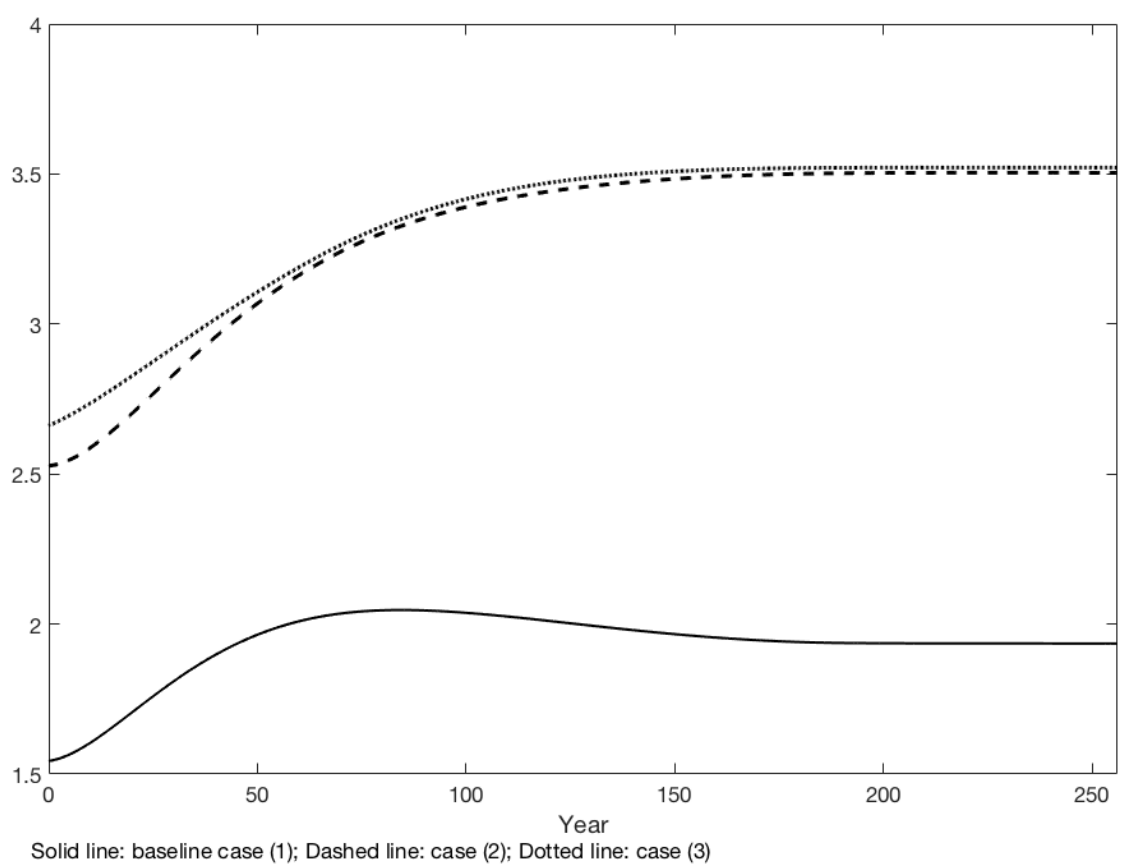

Figure B.1: Transition Dynamics of Aggregate composite consumption

Notes: This figure depicts the transition dynamics of aggregate composite consumption. Solid lines: baseline case (1), dashed lines: case (2) and dotted lines: case (3). Gray lines represent levels in the new stationary equilibrium. The time range in the transition between the old stationary equilibrium and the new stationary equilibrium is 256 years. See assumption 2 and section 3.1 for details on targeting the secular transition. 

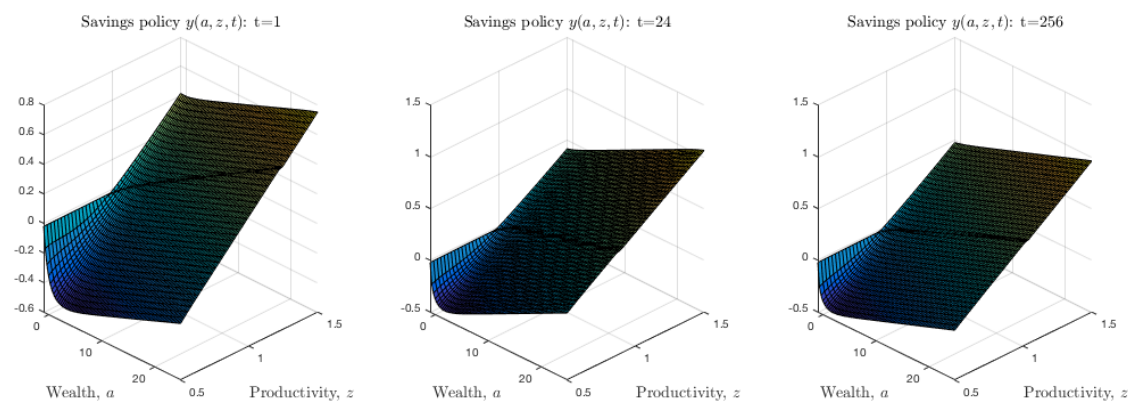

Figure B.2: Savings Policy in Selected Periods of the Transition Dynamics - Case (1)

Notes: This figure depicts the savings policy, $y(a, z, t)$, in the baseline case (1) over the grid of wealth, $a$, and labor productivity, $z$, for $t=1$, the transition beginning; $t=24$, when interest rate peaks; and $t=256$, the new stationary equilibrium in panels $\mathrm{A}, \mathrm{B}$ and $\mathrm{C}$, respectively.
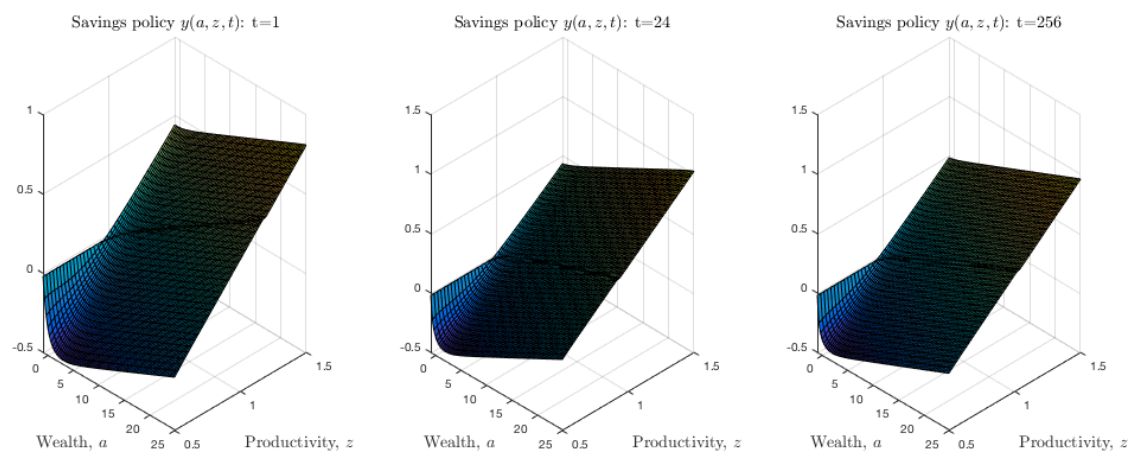

Figure B.3: Savings Policy in Selected Periods of the Transition Dynamics - Case (2)

Notes: This figure depicts the savings policy, $y(a, z, t)$, in case (2) over the grid of wealth, $a$, and labor productivity, $z$, for $t=1$, the transition beginning; $t=24$, when interest rate peaks; and $t=256$, the new stationary equilibrium in panels $\mathrm{A}, \mathrm{B}$ and $\mathrm{C}$, respectively. 

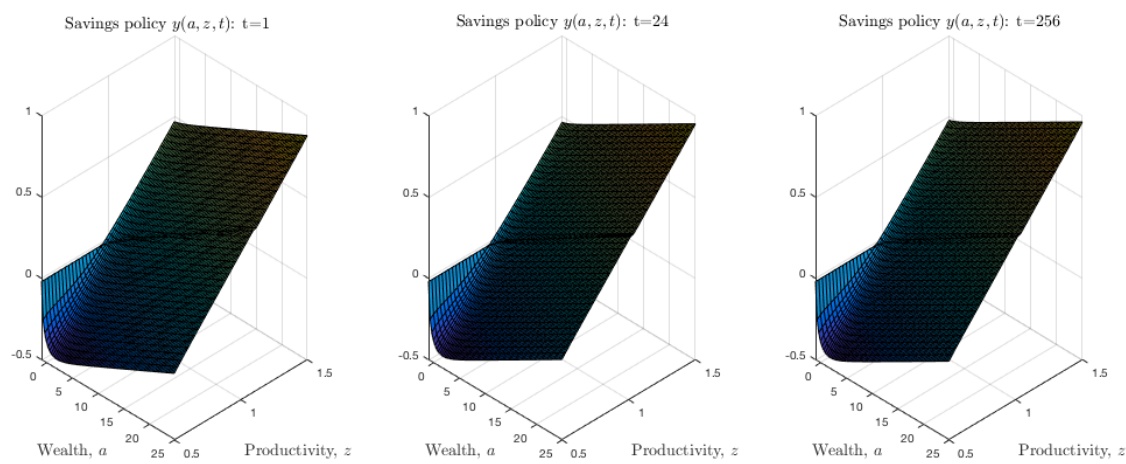

Figure B.4: Savings Policy in Selected Periods of the Transition Dynamics - Case (3)

Notes: This figure depicts the savings policy, $y(a, z, t)$, in case (3) over the grid of wealth, $a$, and labor productivity, $z$, for $t=1$, the transition beginning; $t=24$, when interest rate peaks; and $t=256$, the new stationary equilibrium in panels $\mathrm{A}, \mathrm{B}$ and $\mathrm{C}$, respectively.

Density $g(a, z)$ : baseline case $(1)$

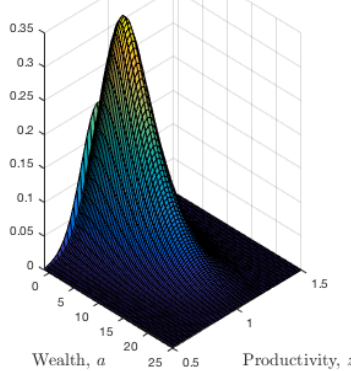

Density $g(a, z)$ : case (2)

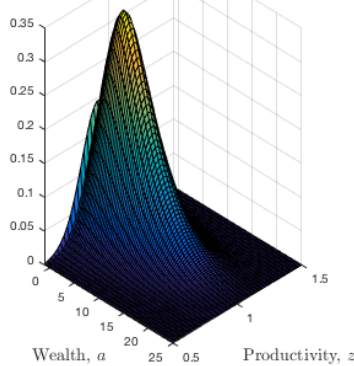

Density $g(a, z)$ : case $(3$

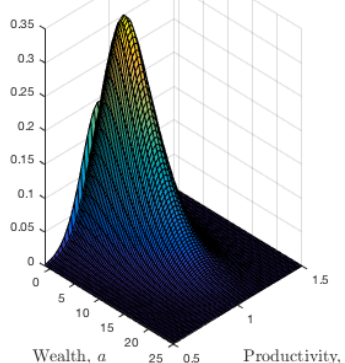

Figure B.5: Density in the New Stationary Equilibrium

Notes: This figure depicts the density in the new stationary equilibrium, $\mathfrak{g}(a, z)$, in the baseline case (1) over the grid of wealth, $a$, and labor productivity, $z$, for the baseline model case (1), case (2) and case (3) in panels A, B and C, respectively. 

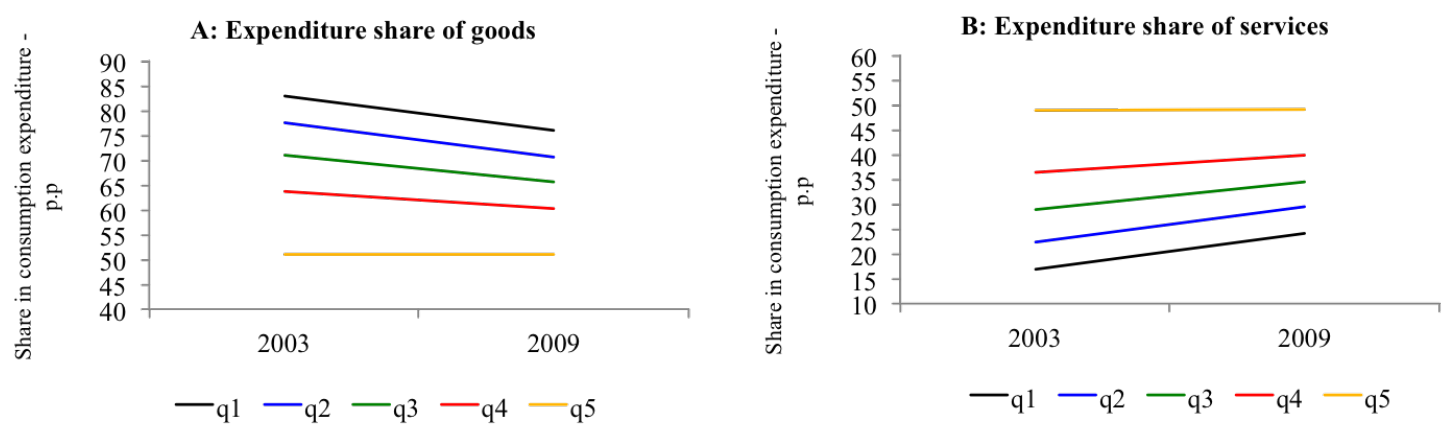

Figure B.6: Engel's Law According to Brazilian Data

Notes: This figure depicts the sectoral shares of households grouped by income quintiles with brazilian data from reported expenditures in the interviews of the POF-IBGE for goods (agriculture and manufacturing) and services in 2003 and 2009.

\section{B.2}

\section{Proofs and derivations}

\section{Proof of Proposition 1}

The proof relies heavily on technical results presented in Acemoglu and Jensen (2012) Acemoglu and Jensen (2015). Before the proof itself, it worths to highlight necessary assumptions we omitted in the main text of section 2 on the model setup to make it more straight to the point and to spare the reader from technicalities that are not the core of the text.

Remind the intertemporal problem (2). A strategy is feasible if it satisfies $\Gamma$ in (2) and it is optimal, $\mathbf{y}^{*}$, if it is a solution to (2), which is ensured by the next standard assumption:

Assumption A.1. For each household, z follows a continuous-time analogue of a Markov process, so that its transition function has the Feller property, $X$ and $Z$ are compact, $\nu$ is bounded and continuous, and $\Gamma$ is continuous with nonempty and compact values.

REMARK A.1. A transition function has the Feller property if the associated Markov operator maps the set of bounded continuous functions into itself.

As it is well known, assumption 1 holds as $z \in Z$, such that $Z=[\underline{z}, \bar{z}] \subseteq$ $\mathbb{R}$, follows an Ornstein-Uhlenbeck process and $X=[\underline{a}, \bar{a}] \subseteq \mathbb{R}$, which ensures compactness of productivities and actions. Moreover, both $\nu$ and aggregate production function, $Y$, feature standard continuity conditions.

Now let's refer to the stationary equilibrium and introduce another necessary assumption to the poof of the existence of stationary equilibrium. 
In a stationary equilibrium, each household faces a constant time path of aggregates, $Q^{*}$, and solves a stationary dynamic programming problem as follows, by rewriting the HJB (27):

$$
\begin{aligned}
v\left(\hat{a}, z, \mathrm{Q}^{*}, \boldsymbol{\theta}\right) & =\max _{\hat{y} \in \Gamma(\hat{a}, z, \hat{\mathrm{Q}}, \boldsymbol{\theta})} \frac{1}{\hat{\rho}}\left[\nu\left(\hat{a}, \hat{y}, z, \mathrm{Q}^{*}, \boldsymbol{\theta}\right)+D_{\hat{a}} v\left(\hat{a}, z, \mathrm{Q}^{*}, \boldsymbol{\theta}\right) y\left(\hat{a}, z, \mathrm{Q}^{*}, \boldsymbol{\theta}\right)\right. \\
& \left.+D_{z} v\left(\hat{a}, z, \mathrm{Q}^{*}, \boldsymbol{\theta}\right) \mu(z)+\frac{1}{2} D_{z z} v\left(\hat{a}, z, \mathrm{Q}^{*}, \boldsymbol{\theta}\right) \sigma^{2}(z)\right]
\end{aligned}
$$

This functional equation has a unique solution $\hat{v}$ under assumption A.1. Note that with the baseline aggregator (14), assumption 1 is sufficient to guarantee the existence of an equilibrium due to the "convexifying" effect of set-valued integration (see assumption 2 and theorem 1 in Acemoglu and Jensen (2012) Acemoglu and Jensen (2015) for the proof).

For a given $v$, the stationary policy correspondence is:

$$
\begin{aligned}
\Phi\left(\hat{a}, z, \hat{\mathrm{Q}}^{*}, \boldsymbol{\theta}\right)= & \arg \max _{\hat{y} \in \Gamma(\hat{a}, z, \hat{\mathrm{Q}}, \boldsymbol{\theta})} \frac{1}{\hat{\rho}}\left[\nu\left(\hat{a}, \hat{y}, z, \mathrm{Q}^{*}, \boldsymbol{\theta}\right)+D_{\hat{a}} v\left(\hat{a}, z, \mathrm{Q}^{*}, \boldsymbol{\theta}\right) y\left(\hat{a}, z, \mathrm{Q}^{*}, \boldsymbol{\theta}\right)\right. \\
& \left.+\quad D_{z} v\left(\hat{a}, z, \mathrm{Q}^{*}, \boldsymbol{\theta}\right) \mu(z)+\frac{1}{2} D_{z z} v\left(\hat{a}, z, \mathrm{Q}^{*}, \boldsymbol{\theta}\right) \sigma^{2}(z)\right]
\end{aligned}
$$

Note that when the idiosyncratic shock process $z_{t}$ is stationary, $\mathfrak{g}(\hat{a}, z)$ in definition 3 is an invariant distribution for this decision problem ${ }^{1}$. The following assumption $^{2}$ is imposed to ensure the existence of such invariant distributions and stationary strategies:

Assumption A.2. $X$ is a lattice; the graph of $\Gamma(., z, Q, \boldsymbol{\theta})$ is a sublattice of $X \times X$ and given any $z, \hat{Q}$ and $\boldsymbol{\theta}, \nu\left(\hat{a}, \hat{y}, z, \mathrm{Q}^{*}, \boldsymbol{\theta}\right)$ is supermodular in $(\hat{a}, \hat{y})$.

REMARK A.2. $X$ is a lattice if for any two elements $\hat{a}^{1}, \hat{a}^{2} \in X$, both the supremum $\hat{a}^{1} \vee \hat{a}^{2} \in X$ and the infimum $\hat{a}^{1} \wedge \hat{a}^{2} \in X$. Fixing and supressing $z, \hat{Q}$ and $\boldsymbol{\theta}$, the graph of $\Gamma$ is a sublattice of $X \times X$ if for all $\hat{a}^{1}, \hat{a}^{2} \in X$, $\hat{y}^{1} \in \Gamma\left(\hat{a}^{1}\right)$ and $\hat{y}^{2} \in \Gamma\left(\hat{a}^{2}\right)$ imply $\hat{y}^{1} \wedge \hat{y}^{2} \in \Gamma\left(\hat{y}^{1} \wedge \hat{y}^{2}\right)$ and $\hat{y}^{1} \vee \hat{y}^{2} \in \Gamma\left(\hat{y}^{1} \vee \hat{y}^{2}\right)$. At last, $\nu$ is supermodular in $(\hat{a}, \hat{y})$ if for all $\hat{a}^{1}, \hat{a}^{2} \in X$ and $\hat{y}^{1}, \hat{y}^{2} \in X$, $\nu\left(\hat{a}^{1} \vee \hat{a}^{2}, \hat{y}^{1} \vee \hat{y}^{2}\right)+\nu\left(\hat{a}^{1} \wedge \hat{a}^{2}, \hat{y}^{1} \wedge \hat{y}^{2}\right) \geq \nu\left(\hat{a}^{1}, \hat{y}^{1}\right)+\nu\left(\hat{a}^{2}, \hat{y}^{2}\right)$.

The statement $X$ is a lattice holds trivially $(X \subseteq \mathbb{R}-$ one-dimensional action set). The graph of $\Gamma(., z, Q, \theta)$ is a sublattice of $X \times X$ if and only if $\Gamma$ is ascending (or increasing in the strong set order ${ }^{3}$ ) in $\hat{a}$, which is staightfor-

\footnotetext{
${ }^{1}$ See Appendix B in Acemoglu and Jensen (2012) Acemoglu and Jensen (2015) for further details.

${ }^{2}$ It is based on assumption 3 in Acemoglu and Jensen (2012) Acemoglu and Jensen (2015).

${ }^{3}$ That is, for all for all $\hat{a}^{2} \geq \hat{a}^{1}$ in $X, \hat{y}^{1} \in \Gamma\left(\hat{a}^{1}\right)$ and $\hat{y}^{2} \in \Gamma\left(\hat{a}^{2}\right)$ imply that $\hat{y}^{1} \wedge \hat{y}^{2} \in \Gamma\left(\hat{a}^{1}\right)$ and $\hat{y}^{1} \vee \hat{y}^{2} \in \Gamma\left(\hat{a}^{2}\right)$.
} 
ward to verify in this one-dimensional case and implies $\Phi$ will be ascending in $\hat{a}^{4}$ Hence, we have $\hat{y}^{1} \wedge \hat{y}^{2} \in \Phi\left(\hat{a}^{1}, z, \mathrm{Q}^{*}, \boldsymbol{\theta}\right)$ and $\hat{y}^{1} \vee \hat{y}^{2} \in \Phi\left(\hat{a}^{2}, z, \mathrm{Q}^{*}, \boldsymbol{\theta}\right)$ for all $\hat{a}^{2} \geq \hat{a}^{1}$ in $X, \hat{y}^{1} \in \Phi\left(\hat{a}^{1}, z, \mathrm{Q}^{*}, \boldsymbol{\theta}\right)$ and $\hat{y}^{2} \in \Phi\left(\hat{a}^{2}, z, \mathrm{Q}^{*}, \boldsymbol{\theta}\right)$ - it means, for example, higher past savings will increase current savings. Furthermore, note that we have $\nu(\hat{a}, \hat{y}, z, \hat{\mathrm{Q}}, \theta)=\tilde{\nu}\left(w(Q) z+\left(r(Q)-\psi_{d}\right) \hat{a}-\hat{y}\right)$. Thus, $\nu$ is supermodular in $(\hat{a}, \hat{y})$ if and only if the individual instantaneous utility function $\tilde{\nu}$ is concave. For the CRRA utility in our model it is clear, since it is twice-differentiable: $D_{\hat{a}, \hat{y}}^{2} \nu=-\left(r(Q)-\psi_{d}\right) \tilde{\nu}^{\prime \prime} \geq 0$ since $\tilde{\nu}^{\prime \prime}=-\varsigma\left(\hat{w}(Q) z+\left(r(Q)-\psi_{d}\right) \hat{a}-\hat{y}\right)^{-(1+\varsigma)} \leq 0$ for $\varsigma>0 .^{5}$

In our multi-sector model, each household faces a constant time path of aggregates, $\mathbf{Q}^{*}$, and sets the stationary policy correspondence, $\Phi^{6}$, in the first-stage decision (intertemporal) problem. In the second-stage decision (intratemporal) problem, each household takes $\left\{Q^{*}, \hat{y}^{*}\right\}$ as given and solves the static programming problem (3):

$$
\chi\left(\hat{a}^{*}\right)=\min _{\left\{\hat{c}_{j}\right\}_{j \in J}: u\left(\hat{c}_{g}, \hat{c}_{s}\right) \leq \bar{u}} \sum_{j \in J} p_{j} \hat{c}_{j},
$$

where $\chi\left(\hat{a}^{*}\right)=\left\{e\left(\mathbf{p}, \bar{u}_{i}\right) \in[0, \bar{e}]: \hat{e}=\hat{w}(Q) z+\left(r\left(\hat{Q}^{*}\right)-\psi_{d}\right) \hat{a}-\hat{y}\right\}$, so that it is continuous with nonempty and compact values by assumption 1 .

This correspondence denotes the value function (total consumption expenditure) of the intratemporal problem, which, in turn, gives rise to the sectoral consumption choices characterized by the following correspondence:

$$
\Lambda(\mathbf{p}, \hat{e})=\arg \min _{\left\{\hat{c}_{j}\right\}_{j \in J}: u\left(\hat{c}_{g}, \hat{c}_{s}\right) \leq \bar{u}} \sum_{j \in J} p_{j} \hat{c}_{j},
$$

where $\Lambda(\mathbf{p}, \hat{e})=\left\{\mathbf{c}(\mathbf{p}, \hat{e}) \in C: \hat{e}=\mathbf{p}_{\mathbf{J}}^{\prime} \cdot \hat{\mathbf{c}},\right\}$ is compact, convex valued and upper hemicontinuous. Note that the scalar $c$ denotes the consumption composite, which differs from the vector $\mathbf{c}$, which represents the bundle containing $J$ sectoral consumption demands. In addition, $\mathbf{p}_{\mathbf{J}}$ denotes the vector containing $J$ sectoral prices.

When assumptions $1-2$ in the main text and assumptions A.1-A.2 are combined, our multi-sector Bewley-Aiyagari economy always has a stationary dynamic equilibrium and least and greatest equilibrium aggregates in a set of equilibrium aggregates, $\mathcal{E}(\hat{a})$, are well defined. Thus, we now can associate the static equilibrium to the stationary dynamic equilibrium by combining

${ }^{4}$ As proved in theorem B2 in Appendix B in Acemoglu and Jensen (2015).

${ }^{5}$ For more details and definitions on lattice, sublattice, ascending correspondence and supermodularity, see assumption 3 in Acemoglu and Jensen (2012) Acemoglu and Jensen (2015) and Topkis (1998).

${ }^{6} \Phi \in \mathbf{\Phi}\left(\hat{Q}^{*}\right)$, the set of optimal strategies, such that $\mathbf{\Phi}\left(\hat{Q}^{*}\right) \subseteq X$. 
assumptions $1-2$, A.1-A.2; and $\chi\left(\hat{a}^{*}\right), \Lambda(\mathbf{p}, \hat{e})$ in Bewley-Aiyagari multisector economies.

Now the proof of proposition 1 . It is only a sketch since theorem 2 in Acemoglu and Jensen (2012) Acemoglu and Jensen (2015) already proved existence by following Kakutani's fixed-point theorem in a standard one-sector model, and also that the set of equilibrium aggregates will be compact and a least and a greatest equilibrium aggregate will always exist. To this respect, we only add a simple step that refers to a multi-sector environment for any $J \geq 2$ (number of sectors).

From theorem B2 in Appendix B in Acemoglu and Jensen (2012) Acemoglu and Jensen (2015), the household's stationary policy correspondence $\Phi: X^{h} \times Z^{h} \times\{Q\} \times \Theta^{h} \rightarrow 2^{X^{h}}$ is compact and convex valued, continuous and ascending in $\hat{a}^{h}$ for every $h$. Thus, to prove that there exists a static equilibrium associated to a dynamic stationary equilibrium hinges upon showing that there exists correspondences $\chi\left(\left(\hat{a}^{h}\right)^{*}\right)=\hat{e}^{h}$ and $\Lambda\left(\mathbf{p}, \hat{e}^{h}\right)=\left(\hat{\mathbf{c}}^{h}\right)^{*}$ that are also convex valued, continuous and ascending in $\hat{a}^{h}$.

REMARK A.3. $\hat{H}$ is an upper hemicontinuous and convex-valued correspondence that maps a compact and convex subset of the reals into itself: $\hat{H}(Q, \boldsymbol{\theta})=\{\tilde{H}(\hat{a}) \in \mathbb{R}: \hat{a} \in G(Q, \boldsymbol{\theta}) \forall h\}$, where $\hat{a}$ is a random variable and $\tilde{H}$ maps distributions into the reals. $G$ is the non-empty valued and upper hemicontinuous joint (for every $h$ ) invariant distribution correspondence $G=\left(G^{h}\right)_{h \in[0,1]}: \mathcal{Q} \times \Theta \rightarrow 2^{\prod_{h \in[0,1]} \mathcal{P}\left(X^{h}\right)}$, where $G^{h}(Q, \boldsymbol{\theta})=$ $\left\{g_{z^{h}} \in \mathcal{P}\left(X^{h}\right): g_{z^{h}} \in T_{Q, a}^{*}\right\}$ such that $T_{Q, a}^{*}$ is the adjoint Markov correspondence induced by the the agents' stationary policy correspondences $\Phi{ }^{7}$

First, consider $\chi^{h}: 2^{X^{h}} \rightarrow 2^{X^{h}}$, which associates with every $\Phi$ the total consumption expenditure, $\hat{e}^{h}$. Since $\Phi$ is compact, convex valued and continuous and $\chi$ is continuous with compact values by assumption 1 , then $\chi \circ \Phi$ is compact, convex valued and continuous. Moreover, by fixing $z^{h} \in Z^{h}$ and $Q^{*} \in \mathcal{Q}, \chi$ is trivially ascending in $\hat{a}$ since $\hat{a}^{h} \in X^{h}, X^{h} \subseteq \mathbb{R}$, which follows directly from Topkis's theorem (Topkis, 1998). Second, consider $\Lambda: P \times X^{h} \rightarrow$ $2^{C^{h}}$, the consumer's demand correspondence, which associates with every pair $\left(\mathbf{p}, \hat{e}^{h}\right) \in P \times X^{h}$ the non-empty set of demand bundles $\hat{\mathbf{c}}^{h}\left(\mathbf{p}, \hat{e}^{h}\right) \in C^{h}$, where $C^{h} \subseteq \mathbb{R}^{J}$ is assumed to be compact and convex. Then since utility function, $\tilde{\nu}$, is continuous, increasing, and concave, there exists a demand correspondence $\Lambda$ which is convex valued and upper hemicontinuous (See, e.g., Debreu, 1959). Now, fix $\mathbf{p} \in P$. $\hat{e}^{h}=\mathbf{p}_{\mathbf{J}}^{\prime} \cdot \hat{\mathbf{c}}^{h}$ is supermodular in $\hat{\mathbf{c}}^{h}$. Hence, $\Phi$ is ascending in $\hat{e}^{h}$ by Topkis's theorem and, since $\chi$ is ascending in $\hat{a}^{h}$, it follows that

\footnotetext{
${ }^{7}$ See the proof of theorem 5 in Acemoglu and Jensen (2012) Acemoglu and Jensen (2015)
} for further details. 
so is $\Lambda\left(\mathbf{p}, \chi\left(\left(\hat{a}^{h}\right)^{*}\right)\right)$. By considering $\Lambda\left(\mathbf{p}, \chi\left(\left(\hat{a}^{h}\right)^{*}\right)\right)$ and given $\hat{H}\left(Q^{*}, \boldsymbol{\theta}\right)$, the upper hemicontinuous and convex-valued correspondence that pins down the stationary equilibrium aggregate (fixed-point), $Q^{*} \in \hat{H}\left(Q^{*}, \boldsymbol{\theta}\right)$, it then sets $\left\{c_{j}^{h}\left(\mathbf{p}, \chi\left(\left(\hat{a}^{h}\right)^{*}\right)\right)\right\} \forall h, j$, for given $\left\{Q^{*},\left(\hat{a}^{h}\right)^{*}\right\}$ in stationary equilibrium. Q.E.D.

\section{Derivation of assumption 1}

From Marshallian demand of services (equation (4)), the solution of each household problem is interior if parameters and the income distribution are such that household expenditure of the poorest household exceeds a minimum nonnegative level. That is, for all $h \in \mathcal{H}=[0,1]$, household consumption expenditure of services is positive. It means in our case that total expenditure exceeds a minimum level.

$$
\begin{aligned}
p_{s, t} \hat{\bar{c}}_{s} & \leq\left[\varphi_{s} p_{s, t}^{1-\varepsilon}\left(\sum_{i=g, s} \frac{p_{i, t}^{\varepsilon} \bar{c}_{i}}{\varphi_{i}}\right)\right]+\left(\frac{\varphi_{s} p_{s, t}^{1-\varepsilon}}{\sum_{i=g, s} \varphi_{i} p_{i, t}^{1-\varepsilon}}\right) \min _{h \in \mathcal{H}} \hat{e}_{t}^{h} \\
\min _{h \in \mathcal{H}} \hat{e}_{t}^{h} & \geq \varrho\left(1-p_{s, t}^{1-\epsilon}\right) p_{s, t} \hat{\bar{c}}_{s}-\left(\frac{\varrho}{\varrho-1}\right) p_{g, t} \hat{\bar{c}}_{g} \\
\hat{e}_{t}^{h} & \geq \varrho\left(1-p_{s, t}^{1-\epsilon}\right)\left[p_{s, t} \max \left(-\hat{\bar{c}}_{s}, 0\right)\right]+\left(\frac{\varrho}{\varrho-1}\right)\left[p_{g, t} \max \left(-\hat{\bar{c}}_{g}, 0\right)\right],
\end{aligned}
$$

for all $h \in \mathcal{H}$, where $\varrho \equiv\left[1+\frac{\varphi_{g}}{\varphi_{s}}\left(\frac{p_{g, t}}{p_{s, t}}\right)^{1-\varepsilon}\right]$. We also restrict $\bar{c}_{s}=0$ and we have that $\bar{c}_{g}<0$ for all $t$ and for all household $h \in \mathcal{H}$. Therefore, total expenditure of the poorest household must be higher than the value of food subsistence (multiplied by the factor $\left(\frac{\varrho}{\varrho-1}\right)$ ):

$$
\min _{h \in \mathcal{H}} \hat{e}_{t}^{h} \geq\left(\frac{\varrho}{\varrho-1}\right)\left[p_{g, t} \max \left(-\hat{\bar{c}}_{g}, 0\right)\right]
$$

Achdou et al. (2017) show that $y(\underline{\hat{a}}, \underline{z}, t)=0$. Hence, $y(\underline{\hat{a}}, \underline{z}, t)=0=$ $\hat{w}\left(Q_{t}\right) \underline{z_{t}}+\underline{a_{t}}\left(r\left(Q_{t}\right)-\psi_{d}\right)-e(\underline{\hat{a}}, \underline{z}, t)$, such that $\min _{h \in \mathcal{H}} \hat{e}_{t}^{h} \equiv e(\underline{\hat{a}}, \underline{z}, t)$. Thus:

$$
\hat{w}\left(Q_{t}\right) \underline{z_{t}}+\underline{a_{t}}\left(r\left(Q_{t}\right)-\psi_{d}\right) \geq\left(\frac{\varrho}{\varrho-1}\right)\left[p_{g, t} \max \left(-\hat{\bar{c}}_{g}, 0\right)\right]
$$

which also means in our case that the same lower bound related to the value of food subsistence applies to the income of the poorest household.

Derivation of Euler equation (31)

It applies Achdou et al. (2017) Achdou et al. (2017) in a multi-sector environment and Ornstein-Uhlenbeck process of the idiosyncratic productivity. Differentiate the HJB equation (17) with respect to $a$ (envelope condition) and use 
that $D_{\hat{a}} v(\hat{a}, z, t)=\tilde{\nu}^{\prime}(e(\hat{a}, z, t))$ and $D_{\hat{a} \hat{a}}^{2} v(\hat{a}, z, t)=\tilde{\nu}^{\prime \prime}(e(\hat{a}, z, t)) D_{\hat{a}} e(\hat{a}, z, t)$ to obtain:

$$
\begin{aligned}
\left(\hat{\rho}+\psi_{d}-r_{t}\right) \tilde{\nu}^{\prime}(e(\hat{a}, z, t)) & =\tilde{\nu}^{\prime \prime}(e(\hat{a}, z, t))\left[D_{\hat{a}} e(\hat{a}, z, t) \hat{y}+\dot{e}(\hat{a}, z, t)+D_{z} e(\hat{a}, z, t) \mu(z)+\frac{1}{2} D_{z z} e(\hat{a}, z, t) \sigma^{2}(z)\right] \\
& +\frac{1}{2} \tilde{\nu}^{\prime \prime \prime}(e(\hat{a}, z, t)) D_{z} e(\hat{a}, z, t),
\end{aligned}
$$

where the right-hand side is is simply the expected change of individual marginal utility of consumption expenditure, $\mathbb{E}_{t}\left\{d \nu^{\prime}(e(\hat{a}, z, t))\right\} d t$, which uses uses the extension of Ito's formula to Ornstein-Uhlenbeck diffusion processes. Therefore, the above equation can be written in the standard form of the Euler equation (31). 


\section{B.3 \\ Data}

\section{B.3.1}

\section{NIPA-BEA data}

It follows Herrendorf et al. (2009) Herrendorf et al. (2013). All data are in per capita terms and for the US during 1947-2010. We calculate a per capita quantity by dividing the total quantity by the population size. We take the population size from NIPA Table 7.1: "Selected Per Capita Product and Income Series in Current and Chained Dollars." The construction of final consumption expenditure data is based on standard NIPA tables from the BEA. We use data from the following tables: Table 2.4.3: "Real Personal Consumption Expenditures by Type of Product, Quantity Indexes"; Table 2.4.4. "Price Indexes for Personal Consumption Expenditures by Type of Product" and Table 2.4.5: "Personal Consumption Expenditures by Type of Product".

For the period 1947-2010 and for the available commodities, we obtain annual data on final consumption expenditure, chain-weighted final consumption quantities, and chain-weighted prices from the BEA. Since quantities calculated with the chain-weighted method are not additive, we use the so called cyclical expansion procedure to aggregate quantities that are not available from the BEA (see online Appendix C in Herredorf et al., 2013).

We assign each commodity to one of the two broad sectors goods, and services. Sectors definition follows Herendorf et a. (2013) closely, except for the fact we do not consider Government Consumption Expenditures and we exclude the following categories: U.S. government civilian and military personnel stationed abroad and final consumption expenditures of nonprofit institutions serving households. We define: Goods sector - durable and nondurable goods excluding alcoholic beverages purchased for off-premises consumption. Services sector: services excluding gambling, finance services and social services and religious activities. Yet one has to keep in mind that fringe benefit payments made by an employer are accounted in the NIPA-BEA data, but are not reported in the CE-BLS data.

\section{B.3.2}

\section{CE-BLS data}

The paper uses the CE-BLS survey data after the introduction of the "NEW ID" (Identifier of the consumption unity) in 1986. The data of the years 1986-2010 are obtained from Aguiar and Bils (2015)'s consumption ex- 
penditure database, a pooled cross-section over 25 annual time series aggregated from U.S. quarterly household consumption data covering more than 200 thousand households. Per capita expenditure arises from diving expenditure by the number of people in each consumption unity. In terms of sample selection the results reported here refer to a sample of urban households whose head is more than 25 and less than 64 years of age. Moreover, as the authors, we only include households that have: at least full-year of interview coverage, complete income reporter, no expenditure outliers and that range between 5-95 percentile of before-tax income to eliminate outliers and mitigate any time-varying impact of bottom-coding and top-coding. It then implies that the bottom income group contains the 5-20 percentile groups and the top income group contains the 80-95 percentile groups. This results in a final sample of more than 50 thousands households.

Sectors are defined in accordance with the first chapter of this thesis (see subsection 3.1.1 and Table 33.3 ), which distributes 22 subsectors among agriculture, manufacturing and services and the association to each CPI-BLS subitem. We lump agriculture and manifacturing together in the goods sector in the present paper. In CE-BLS, the following subsectors define the goods sector: food at home, all other transportation, vehicle purchasing, appliances, phones, computers with associated services, men's and womens' clothing, alcoholic beverages, shoes and other apparel, furniture purchasing, tobacco, other smoking, children's clothing; and the services sector: housing, utilities, food away from home, health expenditures including insurance, entertainment fees, admissions, reading, cash contributions, education, personal care, domestic services and childcare, furniture fixtures.

Finally, we split the broad goods sector into durables and nondurables with data from NIPA-BEA for the Stone-geary parameters estimation to feed the model calibration of the extended baseline case and to solve it. Then, for comparison with CE-BLS data, from which we exclude exclude durable expenditure due to mis-measurement errors related specially to reported durable expenditures, we also exclude durable expenditures predicted by the extended baseline model for the calculation of the sectoral expenditure shares in figure $11^{8}$

${ }^{8}$ For CE-BLS data, see Table A.5 in appendix A of the first chapter of this thesis for the correspondence of sectors, expenditure categories and CPI subitens considerending only nondurable expenditure and prices. 


\section{B.4}

\section{Implementation of the estimation of Stone-Geary preferences parameters}

*Baseline model

Now we estimate the parameters of the demand system (5) that represents the baseline model (1) on annual NIPA-BEA data from 1947-2010 (see data appendix $\mathrm{C}$ for details on data construction). We closely follow previous studies in the literature in employing the iterated feasible generalized nonlinear least square to estimate the share equations. This is a standard way of estimating demand systems (Herrendorf et al. (2009) Herrendorf et al., 2013; Moro et al. 2017).

Consider the expenditure share in each sector in equation (5). Given the set of predetermined variables,

$$
\mathbf{x}_{t} \equiv\left(p_{g, t}, p_{s, t}, e_{t}\right)
$$

and given the set of parameters

$$
\boldsymbol{\theta} \equiv\left(\varepsilon, \bar{c}_{g}, \varphi_{g}, \varphi_{s}\right)
$$

we impose $\bar{c}_{s}=0$ following Matsuyama (2016) Matsuyama (2016) and assume each household solves the intratemporal problem (3) for two shares, $\left\{\frac{p_{j, t} c_{j, t}}{e_{t}}\right\}$, for each sector $j=\{g, s\}$. Note we further assume that all households at a point in time face a common set of prices. We maintain this standard assumption, but acknowledge the caveat that prices may vary across households due to, for example, the ability to search.

Since sectoral shares add up to one, the error covariance matrix is singular. Thus, we drop the demand for goods when we do the estimation and we are left with one nonlinear equation to be estimated

$$
s_{s, t} \equiv \frac{p_{s, t} c_{s, t}}{e_{t}}=f_{s}\left(\mathbf{x}_{t} ; \boldsymbol{\theta}\right)+\epsilon_{s}
$$

where $f_{s}$ is the expenditure shares of services as in equation (5); and $\epsilon_{s}$ is the error term assumed to be uncorrelated with the predetermined variables ${ }^{9}$.

Note that the estimation results are not affected by which equation we drop due to the iteration over the parameters of the error covariance matrix. To deal with the issue that three out of our five (if one take into account $\bar{c}_{s}$ )

${ }^{9}$ This demand system falls into the nonlinear seemingly unrelated regression framework and then in general equations are related through the covariance structure of the error terms. Considering that assumption over the error terms, iterating on the feasible generalized nonlinear least square estimator produces a sequence of parameter estimates that converges to the maximum likelihood estimate. 
parameters are constrained (i.e, $\varepsilon \geq 0 \varphi_{j} \geq 0$ and $\sum_{j=g, s} \varphi_{j}=1$ ), we transform the constrained parameters into unconstrained parameters as follows:

$$
\varepsilon=\exp \left\{b_{0}\right\}, \varphi_{g}=\frac{1}{1+\exp \left\{b_{1}\right\}}, \varphi_{s}=\frac{\exp \left\{b_{1}\right\}}{1+\exp \left\{b_{1}\right\}},
$$

where $b_{0}, b_{1} \in(-\infty,+\infty)$.

After estimating the model in terms of the unconstrained parameters, $b_{0}, b_{1}$ and $\bar{c}_{g}$ we transform these back to compute point estimates and standard errors for the original parameters, $\varepsilon, \varphi_{g}, \varphi_{s}$.

\section{Extended baseline model}

We take a two-step procedure following Moro et al. (2017): first, we fix the value of the elasticity of the substitution parameter between durable and nondurable goods by using a priori information from the literature; second, we estimate the rest of the parameter values from the data according to the estimation above for the baseline model (1).

First step - In this step, we fix the value of the parameter $\gamma$, which governs the intratemporal elasticity of substitution between durables and nondurables following the literature. We set the value of $\gamma$ equal to 0.18 , as estimated by Pakos (2011).

Second step - To estimate the rest of the parameters, we first derive equations for the share of each sector in the extended total consumption. Given the the set of (predetermined) variables that are drawn from on annual NIPABEA data from 1947-2010,

$$
\mathbf{x}_{t} \equiv\left(p_{g_{d}, t}, p_{g_{n d}, t}, p_{s, t}, e_{t}\right)
$$

where indexes $\left\{g_{d}, g_{n d}\right\}$ stand for durable goods and nondurable goods, respectively, and given the set of parameters

$$
\boldsymbol{\theta} \equiv\left(\varepsilon, \phi_{g_{d}}, \bar{c}_{g}, \bar{c}_{g_{n d}}, \varphi_{g}, \varphi_{s}\right)
$$

remind that we impose $\bar{c}_{s}=0$ following Matsuyama (2016) Matsuyama (2016) and assume each household solves the intratemporal problem (3) for three shares, $\left\{\frac{p_{f, t} c_{f, t}}{e_{t}}\right\}$, for each sector $f=\left\{g_{d}, g_{n d}, s\right\}$.

Again, since sectoral shares add up to one, the error covariance matrix is singular. Thus, we drop the demand for nondurable goods when we do the 
estimation and we are left with one nonlinear equation to be estimated

$$
\begin{aligned}
s_{s, t} & \equiv \frac{p_{s, t} c_{s, t}}{e_{t}}=l_{s}\left(\mathbf{x}_{t} ; \boldsymbol{\theta}\right)+\epsilon_{s} \\
s_{g_{d}, t} & \equiv \frac{p_{g_{d}, t} c_{g_{d}, t}}{e_{t}}=l_{g_{d}}\left(\mathbf{x}_{t} ; \boldsymbol{\theta}\right)+\epsilon_{g_{d}}
\end{aligned}
$$

where $l_{s}, l_{g_{d}}$ are the expenditure share of agriculture and the expenditure shares of services as in equations (33) $-(34)$; and $\epsilon_{s}, \epsilon_{g_{d}}$ are the error terms assumed to be uncorrelated with the predetermined variables.

Note once more that the estimation results are not affected by which equation we drop due to the iteration over the parameters of the error covariance matrix. To deal with the issue that four out of our seven (if one take into account $\bar{c}_{s}$ ) parameters are constrained (i.e, $\varepsilon \geq 0, \phi_{g_{d}} \leq 1 \varphi_{j} \geq 0$ and $\left.\sum_{j=g, s} \varphi_{j}=1\right)$, we transform the constrained parameters into unconstrained parameters as follows:

$$
\varepsilon=\exp \left\{b_{0}\right\}, \varphi_{g}=\frac{1}{1+\exp \left\{b_{1}\right\}}, \varphi_{s}=\frac{\exp \left\{b_{1}\right\}}{1+\exp \left\{b_{1}\right\}}, \phi_{g_{d}}=\frac{1}{1+\exp \left\{b_{2}\right\}}
$$

where $b_{0}, b_{1}, b_{2} \in(-\infty,+\infty)$.

After estimating the model in terms of the unconstrained parameters, $b_{0}, b_{1}, b_{2}$, and $\bar{c}_{g}$ and $\bar{c}_{g_{n d}}$ we transform these back to compute point estimates and standard errors for the original parameters, $\varepsilon, \phi_{g_{n d}}, \varphi_{g}, \varphi_{s}$. 


\begin{tabular}{|c|c|c|}
\hline & Baseline Model & $\begin{array}{c}\text { Extended } \\
\text { Baseline Model }\end{array}$ \\
\hline$\varepsilon$ & $\begin{array}{c}0.57 * * * \\
(0.04)\end{array}$ & $\begin{array}{c}0.08 * * * \\
(0.02)\end{array}$ \\
\hline$\gamma$ & & 0.18 \\
\hline$\phi_{g_{n d}}$ & & $\begin{array}{c}0.20 * * * \\
(0.03)\end{array}$ \\
\hline $\bar{c}_{g}$ & $\begin{array}{c}-0.64 * * * \\
(0.02)\end{array}$ & $\begin{array}{c}0.004 \\
(0.003)\end{array}$ \\
\hline $\bar{c}_{g_{n d}}$ & & $\begin{array}{c}-0.83 * * * \\
(0.03)\end{array}$ \\
\hline$\varphi_{g}$ & $\begin{array}{c}0.43 * * * \\
(0.01)\end{array}$ & $\begin{array}{c}0.34 * * * \\
(0.01)\end{array}$ \\
\hline$\varphi_{s}$ & $\begin{array}{c}0.57 * * * \\
(0.01)\end{array}$ & $\begin{array}{c}0.66 * * * \\
(0.01)\end{array}$ \\
\hline$\chi^{2}\left(\bar{c}_{g}=0, \bar{c}_{g_{n d}}=0\right)$ & & $1,006.50 * * *$ \\
\hline$\chi^{2}\left(\bar{c}_{g}=0\right)$ & $671.36^{* * *}$ & 1.7 \\
\hline$A I C$ & -399.19 & -793.38 \\
\hline$R M S E_{g}$ & 0.010 & \\
\hline$R M S E_{g_{n d}}$ & & 0.020 \\
\hline$R M S E_{g_{d}}$ & & 0.019 \\
\hline$R M S E_{s}$ & 0.010 & 0.020 \\
\hline
\end{tabular}

Table B.1: Estimation Results with Final Consumption Expenditure - Baseline model and Extended Baseline Model

Notes: $\chi^{2}\left(\bar{c}_{g}=0\right)$ is the Wald Test Statistics for the hypothesis that $\bar{c}_{g}=0$, $\chi^{2}\left(\bar{c}_{g}=0, \bar{c}_{g_{n d}}=0\right)$ is the Wald Test Statistics for the hypothesis that $\bar{c}_{g}$ and $\bar{c}_{g_{n d}}$ are jointly zero. $R M S E_{f}$ is the root mean squared error for j-sector's share equation. Robust standard errors in parentheses. The calculation of standard errors of the constrained parameters is based on the delta method. ${ }^{* * *}$ Significant at the 1 percent level, ${ }^{* *}$ Significant at the 5 percent level and * Significant at the 10 percent level. The first column exhibits the results for the baseline case (1) on final consumption expenditure and price data from NIPA-BEA. The second column exhibits the resuls for the extended baseline case (1) on final consumption expenditure and price data from NIPA-BEA, where goods expenditures and prices are split into durables and nondurables. Data preparation and estimations follows Herrendorf et al. (2009) Herrendorf et al. (2013)'s partial equilibrium approach and methodology. Data is subject to smaller consistency issues due to data source differences with CE-BLS. The estimations results feed the model calibration. See the data appendix for further details on definitions of expenditure sectors. 


\begin{tabular}{ccccc}
\hline & $\begin{array}{c}\text { Estimated Model } \\
\text { partial equilibrium }\end{array}$ & & $\begin{array}{c}\text { Calibrated Model } \\
\text { general equilibrium }\end{array}$ \\
\cline { 2 - 3 }$-p_{g} \bar{c}_{g_{n d}} / \hat{E}$ & 0.28 & 0.06 & 0.28 & 0.06 \\
\hline
\end{tabular}

Table B.2: Targeted Moments - Nonhomotheticity Term of Nondurable Goods Relative To Final Nondurable Consumption Expenditure

Notes: This table reports in the first two columns the ratio of the value of the nondurable goods subsistence relative to aggregate total nondurable expenditure estimated on data from NIPA-BEA that follows Herrendorf et al. (2009) Herrendorf et al. (2013)'s partial equilibrium approach and methodology for 1947 and 2010. Data is subject to smaller consistency issues due to data source differences with CE-BLS. The last two columns exhibit the same moments by the calibrated general equilibrium extended baseline model. See the data appendix for further details on definitions of expenditure sectors. 
Appendix C

Chapter 3

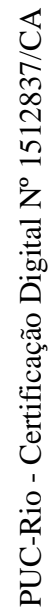




\section{C.1}

\section{Additional Tables}

\begin{tabular}{|c|c|c|c|c|c|}
\hline & $\begin{array}{l}\text { Group } 1 \\
\text { (A) }\end{array}$ & $\begin{array}{l}\text { Group } 2 \\
\text { (B) }\end{array}$ & $\begin{array}{l}\text { Group } 3 \\
\text { (C) }\end{array}$ & $\begin{array}{l}\text { Group } 4 \\
\text { (D) }\end{array}$ & $\begin{array}{l}\text { Group } 5 \\
\text { (E) }\end{array}$ \\
\hline $\ln$ (monthly income 2016) & $\begin{array}{c}1.413 * * * \\
(22.50)\end{array}$ & $\begin{array}{c}1.253 * * * \\
(25.32)\end{array}$ & $\begin{array}{c}1.379 * * * \\
(27.32)\end{array}$ & $\begin{array}{c}1.317 * * * \\
(25.73)\end{array}$ & $\begin{array}{c}1.361 * * * \\
(15.27)\end{array}$ \\
\hline $\ln (\mathrm{age})$ & $\begin{array}{c}2.735 * * * \\
(17.25)\end{array}$ & $\begin{array}{c}2.235 * * * \\
(17.86)\end{array}$ & $\begin{array}{c}2.583 * * * \\
(20.27)\end{array}$ & $\begin{array}{c}2.374 * * * \\
(18.39)\end{array}$ & $\begin{array}{c}2.522 * * * \\
(11.19)\end{array}$ \\
\hline $\ln ($ age) $x \ln ($ monthly income 2016) & $\begin{array}{c}-0.402 * * * \\
(-23.51)\end{array}$ & $\begin{array}{c}-0.359 * * * \\
(-26.59)\end{array}$ & $\begin{array}{c}-0.395 * * * \\
(-28.65)\end{array}$ & $\begin{array}{c}-0.374 * * * \\
(-26.79)\end{array}$ & $\begin{array}{c}-0.388 * * * \\
(-15.95)\end{array}$ \\
\hline male & $\begin{array}{c}-0.169 * * * \\
(-15.55)\end{array}$ & $\begin{array}{c}-0.181 * * * \\
(-21.19)\end{array}$ & $\begin{array}{c}-0.185^{* * *} \\
(-21.42)\end{array}$ & $\begin{array}{c}-0.172^{* * *} \\
(-19.75)\end{array}$ & $\begin{array}{c}-0.179 * * * \\
(-11.68)\end{array}$ \\
\hline white & $\begin{array}{c}0.193 * * * \\
(17.46)\end{array}$ & $\begin{array}{c}0.154 * * * \\
(17.67)\end{array}$ & $\begin{array}{c}0.167 * * * \\
(18.99)\end{array}$ & $\begin{array}{c}0.158^{* * *} \\
(17.84)\end{array}$ & $\begin{array}{c}0.153 * * * \\
(9.86)\end{array}$ \\
\hline high school & $\begin{array}{l}0.084 * * * \\
\quad(5.08)\end{array}$ & $\begin{array}{c}0.082^{* * *} \\
(6.32)\end{array}$ & $\begin{array}{c}0.074 * * * \\
(5.71)\end{array}$ & $\begin{array}{c}0.067 * * * \\
(5.14)\end{array}$ & $\begin{array}{c}0.074 * * * \\
(3.22)\end{array}$ \\
\hline higher education & $\begin{array}{c}-0.192^{* * *} \\
(-10.57)\end{array}$ & $\begin{array}{c}-0.197 * * * \\
(-13.81)\end{array}$ & $\begin{array}{c}-0.217 * * * \\
(-15.2)\end{array}$ & $\begin{array}{c}-0.240^{* * * *} \\
(-16.71)\end{array}$ & $\begin{array}{c}-0.211 * * * \\
(-8.32)\end{array}$ \\
\hline interest rate - vehicles 2016 & $\begin{array}{c}0.0002^{* * *} \\
(4.10)\end{array}$ & $\begin{array}{c}0.0002^{* * *} \\
(5.16)\end{array}$ & $\begin{array}{c}0.0001 * * * \\
(4.15)\end{array}$ & $\begin{array}{c}0.0002 * * * \\
\quad(5.16)\end{array}$ & $\begin{array}{c}0.0001^{* *} \\
(2.28)\end{array}$ \\
\hline interest rate - real estate 2016 & $\begin{array}{c}-0.0001^{* * *} \\
(-3.67)\end{array}$ & $\begin{array}{c}-0.0002 * * * \\
(-5.99)\end{array}$ & $\begin{array}{c}-0.0001 * * * \\
(-5.33)\end{array}$ & $\begin{array}{c}-0.0001 * * * \\
(-4.97)\end{array}$ & $\begin{array}{c}-0.0001 * * \\
(-2.52)\end{array}$ \\
\hline interest rate - credit card debt 2016 & $\begin{array}{l}0.0000 \\
(-0.47)\end{array}$ & $\begin{array}{l}0.000 \\
(1.28)\end{array}$ & $\begin{array}{l}0.000 \\
(1.40)\end{array}$ & $\begin{array}{c}-0.0001 * * * \\
(-2.75)\end{array}$ & $\begin{array}{c}0.000 \\
(-0.86)\end{array}$ \\
\hline interest rate - overdraft debt 2016 & $\begin{array}{c}0.0001 * * \\
(2.01)\end{array}$ & $\begin{array}{c}0.000 \\
(-0.31)\end{array}$ & $\begin{array}{l}0.000 \\
(0.38)\end{array}$ & $\begin{array}{l}0.000 \\
(1.15)\end{array}$ & $\begin{array}{c}0.000 \\
(-0.42)\end{array}$ \\
\hline interest rate - payroll debt 2016 & $\begin{array}{c}-0.0002 * * * \\
(-6.91)\end{array}$ & $\begin{array}{c}-0.0003^{* * *} \\
(-12.03)\end{array}$ & $\begin{array}{c}-0.0003 * * * \\
(-10.24)\end{array}$ & $\begin{array}{c}-0.0003 * * * \\
(-9.68)\end{array}$ & $\begin{array}{c}-0.0003 * * * \\
(-6.74)\end{array}$ \\
\hline $\ln$ (credit card spending 2016) & $\begin{array}{c}-0.026^{* * *} \\
(-14.62)\end{array}$ & $\begin{array}{c}-0.029 * * * \\
(-20.23)\end{array}$ & $\begin{array}{c}-0.029 * * * \\
(-19.83)\end{array}$ & $\begin{array}{c}-0.030 * * * \\
(-20.80)\end{array}$ & $\begin{array}{c}-0.031 * * * \\
(-12.32)\end{array}$ \\
\hline In(balance - real estate 2016) & $\begin{array}{c}-0.076^{* * * *} \\
(-35.33)\end{array}$ & $\begin{array}{c}-0.073 * * * \\
(-43.91)\end{array}$ & $\begin{array}{c}-0.075 * * * \\
(-44.30)\end{array}$ & $\begin{array}{c}-0.078 * * * \\
(-44.99)\end{array}$ & $\begin{array}{c}-0.078^{* * *} \\
(-25.69)\end{array}$ \\
\hline $\ln$ (balance - real vehicles 2016) & $\begin{array}{l}0.003 \\
(1.41)\end{array}$ & $\begin{array}{l}0.002 \\
(1.52)\end{array}$ & $\begin{array}{l}0.002 \\
(1.16)\end{array}$ & $\begin{array}{l}0.001 \\
(0.74)\end{array}$ & $\begin{array}{l}-0.001 \\
(-0.37)\end{array}$ \\
\hline $\ln ($ down payment - real estate 2016) & $\begin{array}{c}-0.034 * * * \\
(-4.22)\end{array}$ & $\begin{array}{c}-0.032^{* * *} \\
(-5.19)\end{array}$ & $\begin{array}{c}-0.026 * * * \\
(-4.25)\end{array}$ & $\begin{array}{c}-0.019 * * * \\
(-3.06)\end{array}$ & $\begin{array}{c}-0.022^{* *} \\
(-2.01)\end{array}$ \\
\hline $\ln ($ down payment - vehicles 2016) & $\begin{array}{l}0.002 \\
(0.47)\end{array}$ & $\begin{array}{l}0.003 \\
(0.71)\end{array}$ & $\begin{array}{l}-0.004 \\
(-1.06)\end{array}$ & $\begin{array}{l}-0.005 \\
(-1.21)\end{array}$ & $\begin{array}{l}0.006 \\
(0.93)\end{array}$ \\
\hline $\ln ($ balance - credit card debt 2016) & $\begin{array}{c}0.023 * * * \\
(11.97)\end{array}$ & $\begin{array}{c}0.026 * * * \\
(17.47)\end{array}$ & $\begin{array}{c}0.028 * * * \\
(18.06)\end{array}$ & $\begin{array}{c}0.028 * * * \\
(18.28)\end{array}$ & $\begin{array}{c}0.029 * * * \\
(10.71)\end{array}$ \\
\hline In(balance - overdraft debt 2016) & $\begin{array}{c}0.005^{* *} \\
(2.17)\end{array}$ & $\begin{array}{c}0.007 * * * \\
(4.35)\end{array}$ & $\begin{array}{c}0.007 * * * \\
(4.24)\end{array}$ & $\begin{array}{c}0.003^{*} \\
(1.92)\end{array}$ & $\begin{array}{c}0.006^{*} \\
(1.89)\end{array}$ \\
\hline In(balance - payroll debt 2016) & $\begin{array}{c}-0.046^{* * *} \\
(-24.05)\end{array}$ & $\begin{array}{c}-0.042^{* * *} \\
(-27.52)\end{array}$ & $\begin{array}{c}-0.044^{* * *} \\
(-28.81)\end{array}$ & $\begin{array}{c}-0.046 * * * \\
(-29.92)\end{array}$ & $\begin{array}{c}-0.044^{* * *} \\
(-16.27)\end{array}$ \\
\hline Fixed Effects & & & State & & \\
\hline Constant & $\begin{array}{c}-9.705^{* * *} \\
(-16.36)\end{array}$ & $\begin{array}{c}-7.791 * * * \\
(-16.66)\end{array}$ & $\begin{array}{c}-9.071 * * * \\
(-19.04)\end{array}$ & $\begin{array}{c}-8.438 * * * \\
(-17.50)\end{array}$ & $\begin{array}{c}-9.138 * * * \\
(-10.81)\end{array}$ \\
\hline Observations & 165,917 & 271,878 & 265,227 & 260,036 & 84,238 \\
\hline Pseudo R-squared & 0.0638 & 0.0655 & 0.0659 & 0.0664 & 0.0675 \\
\hline
\end{tabular}

Table C.1: Propensity Score Matching Logistic Regressions

Notes: This table presents the results of the propensity score matching logistic regression for group $1-5$ in the full database. The dependent variable, treated, is equal to one for individuals in the treatment cluster in group 1, and zero for those in the control cluster in group 1. The treatment sample consists of individuals who qualify for the withdrawals program of the FGTS in 2017, and the control sample consists of all workers that do not qualify for the program. We also exclude individuals/accounts that were dormant or closed or that had no consumption or debt information during the sample period. In addition to the explanatory variables below, we include 27 states categories as fixed effects. T-statistics are presented in parentheses below the coefficient estimates, and $* * *, * *, *$ represent statistical significance at the 1 percent, 5 percent, and 10 percent level, respectively. 


\begin{tabular}{|c|c|c|c|c|c|c|c|c|c|}
\hline & \multicolumn{3}{|c|}{$\begin{array}{l}\text { Group } 1 \\
\text { (A) }\end{array}$} & \multicolumn{3}{|c|}{$\begin{array}{l}\text { Group } 2 \\
\text { (B) }\end{array}$} & \multicolumn{3}{|c|}{$\begin{array}{l}\text { Group } 3 \\
\text { (C) }\end{array}$} \\
\hline & $\begin{array}{l}\text { Credit Card } \\
\text { Spending }\end{array}$ & Payroll Debt & $\begin{array}{l}\text { Credit Card } \\
\text { Debt }\end{array}$ & $\begin{array}{l}\text { Credit Card } \\
\text { Spending }\end{array}$ & Payroll Debt & $\begin{array}{c}\text { Credit Card } \\
\text { Debt }\end{array}$ & $\begin{array}{c}\text { Credit Card } \\
\text { Spending }\end{array}$ & Payroll Debt & $\begin{array}{l}\text { Credit Card } \\
\text { Debt }\end{array}$ \\
\hline$\beta_{0}^{g}$ & $\begin{array}{c}0.030^{* * *} \\
(3.55)\end{array}$ & $\begin{array}{l}0.003 \\
(0.15)\end{array}$ & $\begin{array}{l}-0.001 \\
(-0.22)\end{array}$ & $\begin{array}{l}0.004 \\
(0.58)\end{array}$ & $\begin{array}{l}0.001 \\
(0.03)\end{array}$ & $\begin{array}{l}-0.005 \\
(-0.86)\end{array}$ & $\begin{array}{c}0.016^{* *} \\
(2.37)\end{array}$ & $\begin{array}{l}0.019 \\
(0.92)\end{array}$ & $\begin{array}{l}0.002 \\
(0.26)\end{array}$ \\
\hline$\beta_{1}^{g}$ & $\begin{array}{c}0.039 * * * \\
(4.21)\end{array}$ & $\begin{array}{l}0.011 \\
(0.45)\end{array}$ & $\begin{array}{l}0.004 \\
(0.64)\end{array}$ & $\begin{array}{l}0.013^{*} \\
(1.69)\end{array}$ & $\begin{array}{l}-0.004 \\
(-0.22)\end{array}$ & $\begin{array}{c}-0.002 \\
(-0.3)\end{array}$ & $\begin{array}{c}0.018^{* *} \\
(2.32)\end{array}$ & $\begin{array}{l}0.014 \\
(0.63)\end{array}$ & $\begin{array}{l}0.001 \\
(0.15)\end{array}$ \\
\hline$\beta_{2}^{g}$ & $\begin{array}{c}0.030^{* * * *} \\
(3.52)\end{array}$ & $\begin{array}{l}0.006 \\
(0.24)\end{array}$ & $\begin{array}{l}0.013^{*} \\
(1.73)\end{array}$ & $\begin{array}{l}0.011 \\
(1.49)\end{array}$ & $\begin{array}{l}-0.025 \\
(-1.34)\end{array}$ & $\begin{array}{l}0.004 \\
(0.58)\end{array}$ & $\begin{array}{l}0.012 \\
(1.61)\end{array}$ & $\begin{array}{l}-0.004 \\
(-0.19)\end{array}$ & $\begin{array}{l}0.010 \\
(1.33)\end{array}$ \\
\hline$\beta_{3}^{g}$ & $\begin{array}{c}0.027 * * * \\
(3.12)\end{array}$ & $\begin{array}{c}-0.062^{\star * *} \\
(-2.34)\end{array}$ & $\begin{array}{c}-0.015^{*} \\
(-1.91)\end{array}$ & $\begin{array}{c}0.020^{* * *} \\
(2.66)\end{array}$ & $\begin{array}{c}-0.053 * 3 * * \\
(-2.64)\end{array}$ & $\begin{array}{l}0.002 \\
(0.28)\end{array}$ & $\begin{array}{c}0.016^{* *} \\
(2.20)\end{array}$ & $\begin{array}{l}-0.017 \\
(-0.71)\end{array}$ & $\begin{array}{l}0.006 \\
(0.73)\end{array}$ \\
\hline$\beta_{4}^{g}$ & $\begin{array}{c}0.045^{\star * *} \\
(4.70)\end{array}$ & $\begin{array}{c}-0.089 * * * \\
(-3.17)\end{array}$ & $\begin{array}{c}-0.022^{* * *} \\
(-2.62)\end{array}$ & $\begin{array}{c}0.023 * * * \\
(2.91)\end{array}$ & $\begin{array}{c}-0.098^{* * *} \\
(-4.86)\end{array}$ & $\begin{array}{c}-0.025^{* * *} \\
(-3.37)\end{array}$ & $\begin{array}{c}0.020^{* * *} \\
(2.56)\end{array}$ & $\begin{array}{l}-0.026 \\
(-1.05)\end{array}$ & $\begin{array}{l}0.012 \\
(1.45)\end{array}$ \\
\hline$\beta_{5}^{g}$ & $\begin{array}{l}0.052^{2 * * *} \\
(5.34)\end{array}$ & $\begin{array}{c}-0.102^{* * *} \\
(-3.48)\end{array}$ & $\begin{array}{c}-0.025 * * * \\
(-2.88)\end{array}$ & $\begin{array}{c}0.035^{* * *} \\
(4.13)\end{array}$ & $\begin{array}{c}-0.128^{* * *} \\
(-6.06)\end{array}$ & $\begin{array}{c}-0.025 * * * \\
(-3.36)\end{array}$ & $\begin{array}{c}0.027 * * * \\
(3.33)\end{array}$ & $\begin{array}{c}-0.087 * * * \\
(-3.41)\end{array}$ & $\begin{array}{l}-0.018^{* *} \\
(-2.25)\end{array}$ \\
\hline$\beta_{6}^{g}$ & 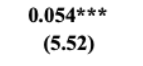 & $\begin{array}{c}-0.085^{\star *} \\
(-2.54)\end{array}$ & $\begin{array}{c}-0.020^{\star *} \\
(-2.29)\end{array}$ & $\begin{array}{c}0.040^{* * *} \\
(4.05)\end{array}$ & $\begin{array}{c}-0.122^{* * *} \\
(-5.38)\end{array}$ & $\begin{array}{c}-0.024^{* * *} \\
(-3.07)\end{array}$ & $\begin{array}{c}0.035^{* * *} \\
(4.35)\end{array}$ & $\begin{array}{c}-0.098^{\star * *} \\
(-3.65)\end{array}$ & $\begin{array}{c}-0.025^{\star \star *} \\
(-3.37)\end{array}$ \\
\hline$\beta_{7}^{g}$ & $\begin{array}{c}0.062^{* * *} \\
(5.72)\end{array}$ & $\begin{array}{c}-0.092^{* * *} \\
(-2.67)\end{array}$ & $\begin{array}{c}-0.016^{*} \\
(-1.75)\end{array}$ & $\begin{array}{c}0.047 * \star * \\
(4.57)\end{array}$ & $\begin{array}{c}-0.123^{* \star *} \\
(-5.24)\end{array}$ & $\begin{array}{c}-0.024^{* * *} \\
(-2.96)\end{array}$ & $\begin{array}{c}0.038^{* * *} \\
(4.37)\end{array}$ & $\begin{array}{c}-0.091^{* * *} \\
(-3.21)\end{array}$ & $\begin{array}{c}-0.026^{* * *} \\
(-3.47)\end{array}$ \\
\hline$\beta_{8}^{g}$ & $\begin{array}{c}0.053^{* * * *} \\
(4.79)\end{array}$ & $\begin{array}{c}-0.083^{* *} \\
(-2.33)\end{array}$ & $\begin{array}{l}-0.010 \\
(-1.05)\end{array}$ & $\begin{array}{c}0.041^{* * *} \\
(3.85)\end{array}$ & $\begin{array}{c}-0.102^{* * *} \\
(-4.01)\end{array}$ & $\begin{array}{c}-0.024 * * * \\
(-3.11)\end{array}$ & $\begin{array}{c}0.043 * * * \\
(4.96)\end{array}$ & $\begin{array}{c}-0.099 * * * \\
(-3.41)\end{array}$ & $\begin{array}{c}-0.026^{* * *} \\
(-3.61)\end{array}$ \\
\hline$\beta_{9}^{g}$ & & & & $\begin{array}{c}0.045^{* * *} \\
(4.31)\end{array}$ & $\begin{array}{c}-0.089^{* * *} \\
(-3.28)\end{array}$ & $\begin{array}{c}-0.026^{* * *} \\
(-3.29)\end{array}$ & $\begin{array}{c}0.038^{* * *} \\
(4.30)\end{array}$ & $\begin{array}{c}-0.083^{* * *} \\
(-2.77)\end{array}$ & $\begin{array}{c}-0.021^{* \star *} \\
(-2.97)\end{array}$ \\
\hline$\beta_{10}^{g}$ & & & & & & & $\begin{array}{c}0.035^{\text {*** }} \\
(4.01)\end{array}$ & $\begin{array}{c}-0.084^{* * *} \\
(-2.73)\end{array}$ & $\begin{array}{c}-0.019^{* * *} \\
(-2.71)\end{array}$ \\
\hline \multicolumn{10}{|l|}{$\beta_{11}^{g}$} \\
\hline \multicolumn{10}{|l|}{$\beta_{12}^{g}$} \\
\hline Constant & $\begin{array}{c}2,079.873 * * * \\
(169.19)\end{array}$ & $\begin{array}{c}11,517.15 * * * \\
(295.89)\end{array}$ & $\begin{array}{c}605.213^{* * *} \\
(87.50)\end{array}$ & $\begin{array}{l}2,140.053 * * * \\
\quad(227.48)\end{array}$ & $\begin{array}{c}11,465.75 * * * \\
(368.71)\end{array}$ & $\begin{array}{c}977.227 * * * \\
(106.02)\end{array}$ & $\begin{array}{l}2,066.926 * * * \\
\quad(216.26)\end{array}$ & $\begin{array}{c}11,519.77 * * * \\
(356.43)\end{array}$ & $\begin{array}{c}973.518^{* * *} \\
(108.07)\end{array}$ \\
\hline$c_{p r e}^{g}, d_{p r e}^{g}$ & $\begin{array}{l}0.053 \\
(1.46)\end{array}$ & $\begin{array}{l}0.013 \\
(0.96)\end{array}$ & $\begin{array}{l}0.002 \\
(0.48)\end{array}$ & $\begin{array}{l}-0.010 \\
(-0.37)\end{array}$ & $\begin{array}{l}0.007 \\
(0.72)\end{array}$ & $\begin{array}{l}0.001 \\
(0.30)\end{array}$ & $\begin{array}{l}0.019 \\
(0.69)\end{array}$ & $\begin{array}{l}0.007 \\
(0.65)\end{array}$ & $\begin{array}{l}0.003 \\
(0.87)\end{array}$ \\
\hline Fixed-effects & \multicolumn{9}{|c|}{ Individual, year-month } \\
\hline Observations & & 606,008 & & & $1,055,275$ & & & $1,080,025$ & \\
\hline
\end{tabular}

Table C.2: Credit Card Spending, Payroll Debt and Credit Card Debt Dynamics Responses - Restricted Sample 


\begin{tabular}{|c|c|c|c|c|c|c|}
\hline & \multicolumn{3}{|c|}{$\begin{array}{l}\text { Group } 4 \\
\text { (D) }\end{array}$} & \multicolumn{3}{|c|}{$\begin{array}{l}\text { Group } 5 \\
\text { (E) }\end{array}$} \\
\hline & $\begin{array}{l}\text { Credit Card } \\
\text { Spending }\end{array}$ & Payroll Debt & $\begin{array}{c}\text { Credit Card } \\
\text { Debt }\end{array}$ & $\begin{array}{l}\text { Credit Card } \\
\text { Spending }\end{array}$ & Payroll Debt & $\begin{array}{l}\text { Credit Card } \\
\text { Debt }\end{array}$ \\
\hline$\beta_{0}^{g}$ & $\begin{array}{c}0.016^{* *} \\
(2.23)\end{array}$ & $\begin{array}{l}0.018 \\
(1.08)\end{array}$ & $\begin{array}{l}0.007 \\
(1.13)\end{array}$ & $\begin{array}{l}0.009 \\
(0.81)\end{array}$ & $\begin{array}{l}0.020 \\
(0.62)\end{array}$ & $\begin{array}{c}-0.018^{*} \\
(-1.72)\end{array}$ \\
\hline$\beta_{1}^{g}$ & $\begin{array}{c}0.019 * * * \\
(2.76)\end{array}$ & $\begin{array}{l}0.009 \\
(0.52)\end{array}$ & $\begin{array}{c}0.012 * \\
(1.81)\end{array}$ & $\begin{array}{l}0.017 \\
(1.29)\end{array}$ & $\begin{array}{l}0.031 \\
(0.91)\end{array}$ & $\begin{array}{l}-0.016 \\
(-1.42)\end{array}$ \\
\hline$\beta_{2}^{g}$ & $\begin{array}{l}0.010 \\
(1.36)\end{array}$ & $\begin{array}{l}0.010 \\
(0.51)\end{array}$ & $\begin{array}{c}0.017 * * \\
(2.40)\end{array}$ & $\begin{array}{l}0.002 \\
(0.14)\end{array}$ & $\begin{array}{l}0.032 \\
(0.87)\end{array}$ & $\begin{array}{l}-0.012 \\
(-0.98)\end{array}$ \\
\hline$\beta_{3}^{g}$ & $\begin{array}{c}0.017 * * \\
(2.20)\end{array}$ & $\begin{array}{l}-0.001 \\
(-0.04)\end{array}$ & $\begin{array}{l}0.007 \\
(1.13)\end{array}$ & $\begin{array}{l}0.013 \\
(1.01)\end{array}$ & $\begin{array}{l}0.009 \\
(0.24)\end{array}$ & $\begin{array}{c}-0.018 \\
(-1.4)\end{array}$ \\
\hline$\beta_{4}^{g}$ & $\begin{array}{c}0.018^{* *} \\
(2.29)\end{array}$ & $\begin{array}{l}-0.014 \\
(-0.65)\end{array}$ & $\begin{array}{l}0.005 \\
(0.82)\end{array}$ & $\begin{array}{l}0.017 \\
(1.37)\end{array}$ & $\begin{array}{l}-0.009 \\
(-0.24)\end{array}$ & $\begin{array}{l}-0.018 \\
(-1.41)\end{array}$ \\
\hline$\beta_{5}^{g}$ & $\begin{array}{c}0.026 * * * \\
(3.20)\end{array}$ & $\begin{array}{c}-0.040^{*} \\
(-1.73)\end{array}$ & $\begin{array}{l}0.004 \\
(0.46)\end{array}$ & $\begin{array}{l}0.019 \\
(1.38)\end{array}$ & $\begin{array}{l}-0.007 \\
(-0.17)\end{array}$ & $\begin{array}{c}-0.021 * \\
(-1.65)\end{array}$ \\
\hline$\beta_{6}^{g}$ & $\begin{array}{c}0.026 * * * \\
(3.18)\end{array}$ & $\begin{array}{c}-0.095 * * * \\
(-3.93)\end{array}$ & $\begin{array}{c}-0.020 * * * \\
(-2.91)\end{array}$ & $\begin{array}{c}0.022 * \\
(1.68)\end{array}$ & $\begin{array}{l}-0.007 \\
(-0.15)\end{array}$ & $\begin{array}{c}-0.023 * \\
(-1.75)\end{array}$ \\
\hline$\beta_{7}^{g}$ & $\begin{array}{c}0.034 * * * \\
(3.96)\end{array}$ & $\begin{array}{c}-0.108 * * * \\
(-4.27)\end{array}$ & $\begin{array}{c}-0.024 * * * \\
(-3.58)\end{array}$ & $\begin{array}{c}0.032 * * \\
(2.27)\end{array}$ & $\begin{array}{l}-0.062 \\
(-1.41)\end{array}$ & $\begin{array}{c}-0.044 * * * \\
(-3.48)\end{array}$ \\
\hline$\beta_{8}^{g}$ & $\begin{array}{c}0.040 * * * \\
(4.23)\end{array}$ & $\begin{array}{c}-0.109 * * * \\
(-4.23)\end{array}$ & $\begin{array}{c}-0.019 * * \\
(-2.51)\end{array}$ & $\begin{array}{c}0.036 * * \\
(2.42)\end{array}$ & $\begin{array}{c}-0.080 * \\
(-1.76)\end{array}$ & $\begin{array}{c}-0.042 * * * \\
(-3.25)\end{array}$ \\
\hline$\beta_{9}^{g}$ & $\begin{array}{c}0.046 * * * \\
(4.67)\end{array}$ & $\begin{array}{c}-0.109 * * * \\
(-4.21)\end{array}$ & $\begin{array}{c}-0.015 * \\
(-1.84)\end{array}$ & $\begin{array}{c}0.039 * * * \\
(2.68)\end{array}$ & $\begin{array}{l}-0.074 \\
(-1.52)\end{array}$ & $\begin{array}{c}-0.042 * * * \\
(-3.18)\end{array}$ \\
\hline$\beta_{10}^{g}$ & $\begin{array}{c}0.046 * * * \\
(4.62)\end{array}$ & $\begin{array}{c}-0.111 * * * \\
(-4.09)\end{array}$ & $\begin{array}{c}-0.017 * * \\
(-2.31)\end{array}$ & $\begin{array}{c}0.038 * * * \\
(2.61)\end{array}$ & $\begin{array}{l}-0.081 \\
(-1.59)\end{array}$ & $\begin{array}{c}-0.042 * * * \\
(-3.06)\end{array}$ \\
\hline$\beta_{11}^{g}$ & $\begin{array}{c}0.047 * * * \\
(4.56)\end{array}$ & $\begin{array}{c}-0.114 * * * \\
(-4.05)\end{array}$ & $\begin{array}{c}-0.016 * \\
(-1.92)\end{array}$ & $\begin{array}{c}0.047 * * * \\
(3.23)\end{array}$ & $\begin{array}{l}-0.077 \\
(-1.49)\end{array}$ & $\begin{array}{c}-0.038 * * * \\
(-2.81)\end{array}$ \\
\hline$\beta_{12}^{g}$ & & & & $\begin{array}{c}0.062^{* * *} \\
(3.98)\end{array}$ & $\begin{array}{l}-0.054 \\
(-1.02)\end{array}$ & $\begin{array}{c}-0.032 * * \\
(-2.36)\end{array}$ \\
\hline Constant & $\begin{array}{l}2,070.036^{* * *} \\
\quad(215.15)\end{array}$ & $\begin{array}{c}11,332.63^{* * * *} \\
(335.24)\end{array}$ & $\begin{array}{c}932.351 * * * \\
(107.94)\end{array}$ & $\begin{array}{l}2,102.546^{* * *} \\
\quad(122.00)\end{array}$ & $\begin{array}{c}11,316.79 * * * \\
(190.07)\end{array}$ & $\begin{array}{l}956.422 * * * \\
\quad(60.69)\end{array}$ \\
\hline$c_{p r e}^{g}, d_{p r e}^{g}$ & $\begin{array}{l}0.015 \\
(0.59)\end{array}$ & $\begin{array}{l}0.016 \\
(1.58)\end{array}$ & $\begin{array}{l}0.003 \\
(0.88)\end{array}$ & $\begin{array}{l}0.032 \\
(0.70)\end{array}$ & $\begin{array}{l}0.025 \\
(1.09)\end{array}$ & $\begin{array}{c}0.003 \\
(-0.70)\end{array}$ \\
\hline \multicolumn{7}{|l|}{ Fixed-effects } \\
\hline Observations & & $1,119,123$ & & & 387,794 & \\
\hline
\end{tabular}

Table C.2: Credit Card Spending, Payroll Debt and Credit Card Debt Dynamics Responses - Restricted Sample (Continued)

Notes: This table shows the credit card spending, payroll debt and credit card debt responses dynamics (equation (3)) in the period from from 2016:05 to 2017:12 using the full sample, restricted to individual holding at least credit card spending and these two debt modalities. $\beta_{s}^{g}$ measures the coefficient estimates of variable $\$ W_{i}^{g} \times 1_{\text {month }}$ and represents the average monthly response in month $s=\{-1,0, \ldots, 12\}$, where $s=-1$ stands for 2016:11, $s=0$ is the announcement month $2016: 12, \ldots$, and $s=12$ is the last month in the withdrawal period of group $g=5$, 2017:12. We define $c_{p r e}^{g} \equiv \sum_{t=-6}^{-1} \beta_{t}^{g}$ and $d_{p r e}^{g} \equiv \frac{1}{6} \sum_{t=-6}^{-1} \beta_{t}^{g}$ as the cumulative consumption response and the average debt modality response, respectively, within the pre-treatment window for group $g$, where $g=\{1,2,3,4,5\}$. Withdrawal periods for each group are in bold. Individual and year-month fixed effects are included, and standard errors are clustered at the individual level. T-statistics are reported in parentheses under the coefficient estimates. ${ }^{* * *}$ Significant at the 1 percent level. ${ }^{* *}$ Significant at the 5 percent level. * Significant at the 10 percent level. 


\begin{tabular}{|c|c|c|c|c|c|}
\hline & $\begin{array}{c}\text { Group } 1 \\
\text { (A) }\end{array}$ & $\begin{array}{c}\text { Group } 2 \\
\text { (B) }\end{array}$ & $\begin{array}{c}\text { Group } 3 \\
\text { (C) }\end{array}$ & $\begin{array}{c}\text { Group } 4 \\
\text { (D) }\end{array}$ & $\begin{array}{c}\text { Group } 5 \\
\text { (E) }\end{array}$ \\
\hline \multicolumn{6}{|c|}{ Consumption } \\
\hline \multicolumn{6}{|c|}{ Panel A: cross sectional test in the matched sample } \\
\hline$\$ W^{g}$ & $\begin{array}{c}0.065 * * * \\
(8.52)\end{array}$ & $\begin{array}{c}0.074 * * * \\
(12.48)\end{array}$ & $\begin{array}{c}0.057 * * * \\
(9.53)\end{array}$ & $\begin{array}{c}0.050 * * * \\
(8.62)\end{array}$ & $\begin{array}{c}0.063 * * * \\
(6.33)\end{array}$ \\
\hline \multicolumn{6}{|c|}{ Panel B: cross sectional test in the full sample } \\
\hline$\$ W^{g}$ & $\begin{array}{c}0.059 * * * \\
(17.37)\end{array}$ & $\begin{array}{c}0.063 * * * \\
(23.76)\end{array}$ & $\begin{array}{c}0.053^{* * *} \\
(20.02)\end{array}$ & $\begin{array}{c}0.046^{* * *} \\
(17.30)\end{array}$ & $\begin{array}{c}0.048^{* * *} \\
(9.99)\end{array}$ \\
\hline \multicolumn{6}{|c|}{ Total Debt } \\
\hline \multicolumn{6}{|c|}{ Panel C: cross sectional test in the matched sample } \\
\hline$\$ W^{g}$ & $\begin{array}{c}-0.072 * * * \\
(-4.16)\end{array}$ & $\begin{array}{c}-0.041^{* * *} \\
(-3.08)\end{array}$ & $\begin{array}{c}-0.042 * * * \\
(-3.03)\end{array}$ & $\begin{array}{c}-0.057 * * * \\
(-4.25)\end{array}$ & $\begin{array}{c}-0.104 * * * \\
(-4.27)\end{array}$ \\
\hline \multicolumn{6}{|c|}{ Panel D: cross sectional test in the full sample } \\
\hline$\$ W^{g}$ & $\begin{array}{c}-0.035^{* * *} \\
(-4.90)\end{array}$ & $\begin{array}{c}-0.043^{* * *} \\
(-7.64)\end{array}$ & $\begin{array}{c}-0.030^{* * *} \\
(-5.27)\end{array}$ & $\begin{array}{c}-0.029 * * * \\
(-5.17)\end{array}$ & $\begin{array}{c}-0.041^{* * *} \\
(-3.96)\end{array}$ \\
\hline
\end{tabular}

Table C.3: Robustness - Cross Sectional Test in the Matched and Full Samples

Notes: This table presents robustness checks of the results shown in Tables $3-4$ and Tables 7-8. We compute the average monthly consumption and total debt during the six months before treatment (2016:06-2016:11), and during the months after treatment (2016:12$2017: 08, \ldots, 2016: 12-2017: 12$, respectively, for each of the five groups of treated workers). Then we compute, as our dependent variables, the difference between the after-treatment average and before-treatment average for each individual. We then regress the dependent variables on the withdrawn amount of each group $g, \$ W^{g}$, in the cross section tests following Bertrand, et al. (2004) . In Panels A and B, we report results on consumption in the matched sample and in the full sample, respetively. Panels $\mathrm{C}$ and D similarly show the results on total debt. 


\section{2}

\section{A simple model of wealthy hand-to-mouth behaviour under an unexpected liquidity shock to the FGTS illiquid assets}

In this Appendix, we provide a two-period consumption model as a simple framework to think of the liquidity shock to the FGTS asset. We provide insights on how it affects allocations to both constrained and unconstrained consumers. Remind that an inactive account of the FGTS represents an unusual illiquid asset for the specialized literature that introduces an additional asset (illiquid) to benchmark (liquid and risk-free) one-asset economies. In this two-asset environment, households may choose to hold (or not to hold) an illiquid asset that typically yields a higher return, but it can only be accessed by paying a transaction cost. In the FGTS case, it is compulsory and yields a lower return.

Households (HHs) live for two periods, $t=1,2$. Preferences over consumption a $t=1,2$ are given by:

$$
v_{1}=u\left(c_{1}\right)+u\left(c_{2}\right),
$$

with no discounting and assume $u^{\prime}>0$ and $u^{\prime \prime}<0$.

The FGTS asset is an illiquid endowment $a$ in $t=1$ that pays off gross return $R_{a}$ in $t=2$, but cannot be accessed at time of the consumption decision in $t=1$. We define $a_{1}=\alpha y_{1}, 0<\alpha<1$, so that it varies proportionally to individual's income in $t=1$ (that is, $\alpha y_{L}$ or $\alpha y_{H}$ ), and $a_{2}=R_{a}\left(\alpha y_{1}\right)$ in $t=2$.

There is a liquid asset $m$ that pays a return $R_{m}=1>R_{a}$. For now, we do not allow the agent to borrow, i.e., a negative position in $m$.

We set period income $y_{2}=\Gamma>1$ and allow two possible values for $y_{1},\left\{y_{L}, y_{H}\right\}$, where $\frac{\Gamma}{(1+\alpha)}>y_{L}>0$ and $y_{H} \geq \frac{\Gamma}{\left(1-R_{a} \alpha\right)}$. We refer to these two cases as "low-income" and "high-income" paths.

Characterization of hand-to-mouth (HtM) behavior concerns the asset position at the time of the $t=1$ consumption decision: i. Non hand-to-mouth (N-HtM): after consuming at $t=1$, holds a positive amount of liquid assets, i.e. $m_{2}>0$ (or, alternatively, $m_{2}$ is negative and higher than the credit limit -indebted N-HtM -, when it is allowed) and $a>0$ and ii. Wealthy hand-tomouth (W-HtM): after consuming at $t=1$, does not hold liquid assets, i.e. $m_{2}=0$ (or, alternatively, $m_{2}$ equals the credit limit, when it is allowed) and $a>0$. For simplicity, there is no poor hand-to-mouth (P-HtM), $m_{2}=0$ and $a=0$, since every treated HH holds $a>0^{1}$.

\footnotetext{
${ }^{1}$ In our empirical analysis, only HHs in the control cluster can be $\mathrm{P}-\mathrm{HtM}$. However, we do not consider them in this model for simplicity.
} 


\section{C.2.1}

\section{Consumption decision at $t=1$ : no-borrowing case}

$$
\begin{aligned}
v_{1} & =\max _{c_{1}, m_{2}} u\left(c_{1}\right)+u\left(m_{2}+a_{2}+\Gamma\right) \\
\text { s.t } & \\
c_{1}+m_{2} & =y_{1} \\
m_{2} & \geq 0
\end{aligned}
$$

The solution is $m_{2}=\max \left\{\frac{y_{1}\left(1-R_{a} \alpha\right)-\Gamma}{2}, 0\right\}$. Therefore, there are the corner and interior solutions. First, suppose $y_{1}=y_{L}$. Thus, the solution is a corner one, $m_{2}=0$, and implies an increasing consumption path, $c_{1}=y_{L}$ and $c_{2}=a_{2}+\Gamma$. Under the low income path, the constraint binds at $t=1$ and the $\mathrm{HH}$ is a W-HtM. Second, if $y_{1}=y_{H}$, the solution is interior and implies a perfectly smooth consumption path $c_{1}=c_{2}=\frac{y_{H}\left(1-R_{a} \alpha\right)+\Gamma}{2}$ because there is no discounting and the interest rate on the liquid asset is 1 . The constraint is not binding and the household is N-HtM with a smooth consumption profile.

\section{C.2.2}

\section{Consumption decision at $t=1$ with an unexpected liquidity shock:}

$$
\begin{gathered}
v_{1}^{s}=\max _{c_{1}^{s}, m_{2}^{s}} u\left(c_{1}^{s}\right)+u\left(m_{2}^{s}+\Gamma\right) \\
\text { s.t } \\
c_{1}^{s}+m_{2}^{s}=y_{1}+a_{1} \\
m_{2}^{s} \geq 0,
\end{gathered}
$$

where the superscript $s$ stands for allocations following the liquidity shock. In this case, since $R_{a}<1$ this problem equals the case of an unexpected income transfer $\tau=a_{1}=\alpha y_{1}$.

The solution is $m_{2}^{s}=\max \left\{\frac{y_{1}(1+\alpha)-\Gamma}{2}, 0\right\}$. Now suppose $y_{1}=y_{L} \cdot{ }^{2}$ Thus, the solution is a corner one, $m_{2}^{s}=0$, and implies an increasing consumption path, $c_{1}^{s}=y_{L}(1+\alpha)$ and $c_{2}^{s}=\Gamma$. Under the low income path, the constraint binds at $t=1$, the $\mathrm{HH}$ is a W-HtM and has MPC of 1 . If $y_{1}=y_{H}$, the solution is

${ }^{2}$ Note that in our exercise to facilitate interpretation we rule out the case where the shock is large enough so that the $\mathrm{W}-\mathrm{HtM}$ becomes $\mathrm{N}-\mathrm{HtM}$ since we impoe the condition $\frac{\Gamma}{(1+\alpha)}>y_{L}>0$. If we relax it, one can show that if $\alpha y_{L} \geq \Gamma-y_{L}$, consumption equals $\frac{y_{L}+a_{1}+\Gamma}{2 a_{1}}$ and and its MPC out of the $a_{1}$ drops to $\frac{-y_{L}+a_{1}+\Gamma}{2 a_{1}}$ which approaches $1 / 2$ as $a_{1}$ increases. 
interior and implies a perfectly smooth consumption path $c_{1}^{s}=c_{2}^{s}=\frac{y_{H}(1+\alpha)+\Gamma}{2}$. The constraint is not binding, the HH is N-HtM and has MPC of 12 .

Therefore, the impact of a positive liquidity shock is the case of the FGTS is equivalent to a positive income shock since there is an underlying income effect following the FGTS liquidity shock related to the fact that $R_{a}<1=R_{m}$. Hence, $v_{1}^{s}>v_{1}$ since we have that $c_{1}^{s}+c_{2}^{s}>c_{1}+c_{2}$ and $m_{2}^{s}>m_{2}$ irrespective of whether the consumer is W-HtM or N-HtM.

\section{2.3}

\section{Consumption decision at $t=1$ with borrowing constraint}

In this case, we allow households to access credit to finance consumption at $t=1$. With a borrowing limit, we substitute the constraint $m_{2} \geq 0$ for $m_{2} \geq-\frac{\phi \Gamma}{R_{b}}$, so that $\underline{m}=\frac{\phi \Gamma}{R_{b}}$ is the credit limit, where $\phi \leq 1$ is a fraction of future income $\Gamma$ and the interest rate on borrowing is $R_{b}>1$. We additionally impose that $R_{b}<\frac{\Gamma}{y_{L}(1+\alpha)}$, which ensures that any HH with the low income path will always borrow a positive amount. In this sense case B.1 can be interpreted alternativelly as a model where borrowing is allowed but $R_{b} \geq \frac{\Gamma}{y_{L}(1+\alpha)}$. That is, credit is sufficiently expensive, so that no HH uses it. We also impose logarithmic utility for simplicity.

Here we have two cases of interior solution. The first is a N-HtM HH under the high income path, $y_{H}$, and chooses to save some of its high income into the liquid asset at $t=1$. Hence, the solution with borrowing is unchanched with respect to interior solution in B.1 and $m_{2}>0$.

HHs under the low income path are indebted, $m_{2} \leq 0$, and the consumption decision follows:

$$
\begin{gathered}
v_{1}=\max _{c_{1}, m_{2}} \log \left(c_{1}\right)+\log \left(R_{b} m_{2}+a_{2}+\Gamma\right) \\
\text { s.t } \\
c_{1}+m_{2}=y_{L} \\
0 \geq m_{2} \geq-\frac{\phi \Gamma}{R_{b}},
\end{gathered}
$$

which has the solution: $m_{2}=\max \left\{-\frac{\Gamma-y_{L}\left(R_{b}-R_{a} \alpha\right)}{2 R_{b}},-\frac{\phi \Gamma}{R_{b}}\right\}$. Since $R_{b}<\frac{\Gamma}{y_{L}(1+\alpha)}$, the $\mathrm{HH}$ always borrows a positive amount. Furthermore, if $R_{b}<\frac{\Gamma(1-2 \phi)}{y_{L}}+R_{a} \alpha$, then the credit limit is binding, $m_{2}=-\frac{\phi \Gamma}{R_{b}}$. The HH is a W-HtM forced to choose $c_{1}=y_{L}+\frac{\phi \Gamma}{R_{b}}$ and $c_{2}=\Gamma(1-\phi)+R_{a} \alpha y_{L}$. Instead, if $\frac{\Gamma}{y_{L}(1+\alpha)}>R_{b} \geq$ $\frac{\Gamma(1-2 \phi)}{y_{L}}+R_{a} \alpha$, the second case of interior solution refers to a indebted N-HtM. Hence, the solution becomes $m_{2}=\frac{y_{L}\left(R_{b}-R_{a} \alpha\right)-\Gamma}{2 R_{b}}$. By borrowing, the HH can perfectly smooth consumption at the level $c_{1}=c_{2}=\frac{y_{L}\left(R_{b}-R_{a} \alpha\right)+\Gamma}{2 R_{b}}$. 


\section{2.4}

\section{Consumption decision at $t=1$ with an unexpected liquidity shock:}

Again, there are two cases of interior solution. The first is a $\mathrm{N}-\mathrm{HtM} \mathrm{HH}$ under the high income path, $y_{H}$, and chooses to save some of its high income into the liquid asset at $t=1$. Hence, the solution with borrowing is unchanched with respect to interior solution in B.1.1 and $m_{2}>0$.

HHs under the low income path are indebted, $m_{2} \leq 0$, and the consumption decision follows:

$$
\begin{gathered}
v_{1}^{s}=\max _{c_{1}, m_{2}} \log \left(c_{1}^{s}\right)+\log \left(R_{b} m_{2}^{s}+\Gamma\right) \\
\text { s.t } \\
c_{1}^{s}+m_{2}^{s}=y_{L}+a_{1} \\
0 \geq m_{2}^{s} \geq-\frac{\phi \Gamma}{R_{b}},
\end{gathered}
$$

which has the solution: $m_{2}^{s}=\max \left\{-\frac{\Gamma-y_{L} R_{b}(1+\alpha)}{2 R_{b}},-\frac{\phi \Gamma}{R_{b}}\right\}$. If $R_{b}<\frac{\Gamma(1-2 \phi)}{y_{L}(1+\alpha)}$, then the credit limit is binding, $m_{2}^{s}=-\frac{\phi \Gamma}{R_{b}}$. Note that it is a stronger condition relative to B.2, without the liquidity shock. The $\mathrm{HH}$ is a W-HtM, has MPC of 1 and then is forced to choose $c_{1}^{s}=y_{L}(1+\alpha)+\frac{\phi \Gamma}{R_{b}}$ and $c_{2}^{s}=\Gamma(1-\phi)$. Instead, if $R_{b} \geq \frac{\Gamma(1-2 \phi)}{y_{L}(1+\alpha)}, 3$ the second case of interior solution refers to a indebted NHtM and has MPC of 12 . Hence, the solution becomes $m_{2}^{s}=\frac{y_{L} R_{b}(1+\alpha)-\Gamma}{2 R_{b}}$. By borrowing, the $\mathrm{HH}$ can perfectly smooth consumption at the level $c_{1}^{s}=c_{2}^{s}=$ $\frac{y_{L} R_{b}(1+\alpha)+\Gamma}{2 R_{b}}$.

In the case we allow for borrowing contraint, we also have that $c_{1}^{s}+c_{2}^{s}>$ $c_{1}+c_{2}$ regardless of whether the consumer is $\mathrm{W}-\mathrm{HtM}$ or N-HtM. Particularly with respect to the indebted N-HtM HHs, since $y_{L} R_{b}(1+\alpha)>y_{L}\left(R_{b}-R_{a} \alpha\right)$, we have that $m_{2}<m_{s}^{s}<0$, which means that the liquidity shock implies a deleveraging effect to this type of HHs.

Table B.1 summarizes the allocations of $c_{1}$ and $m_{2}$ in all cases we presented above.

${ }^{3}$ Alternatively, one can think that if $a_{1}$ is sufficiently large so that this condition holds. 


\begin{tabular}{|c|c|c|c|c|c|c|}
\hline & \multicolumn{3}{|c|}{ Without unexpected liquidity shock } & \multicolumn{3}{|c|}{ With unexpected liquidity shock } \\
\hline & $\mathrm{N}-\mathrm{HtM}$ & W-HtM & Indebted W-HtM & $\mathrm{N}-\mathrm{HtM}$ & W-HtM & Indebted W-HtM \\
\hline \multicolumn{7}{|c|}{ Panel A: No-borrowing case } \\
\hline$c_{1}$ & {$\left[y_{H}\left(1-R_{a}\right)+\Gamma\right] / 2$} & $y_{L}$ & - & {$\left[y_{H}(1+\alpha)+\Gamma\right] / 2$} & $y_{L}(1+\alpha)$ & - \\
\hline $\mathrm{m}_{2}$ & {$\left[y_{H}\left(1-R_{a}\right)-\Gamma\right] / 2$} & 0 & - & {$\left[y_{H}(1+\alpha)-\Gamma\right] / 2$} & 0 & - \\
\hline \multicolumn{7}{|c|}{ Panel B: with borrowing contraint } \\
\hline $\mathrm{c}_{1}$ & {$\left[y_{H}(1+\alpha)+\Gamma\right] / 2$} & $y_{L}+\phi \Gamma / R_{b}$ & {$\left[y_{L}\left(R_{b}-R_{a} \alpha\right)+\Gamma\right] / 2 R_{b}$} & {$\left[y_{H}(1+\alpha)+\Gamma\right] / 2$} & $y_{L}(1+\alpha)+\phi \Gamma / R_{b}$ & {$\left[y_{L} R_{b}(1+\alpha)+\Gamma\right] / 2 R_{b}$} \\
\hline $\mathrm{m}_{\mathrm{*}}$ & {$\left[y_{H}\left(1-R_{a}\right)-\Gamma\right] / 2$} & $-\phi \Gamma / R_{b}$ & {$\left[y_{L}\left(R_{b}-R_{a} \alpha\right)-\Gamma\right] / 2 R_{b}$} & {$\left[y_{H}(1+\alpha)-\Gamma\right] / 2$} & $-\phi \Gamma / R_{b}$ & {$\left[y_{L} R_{b}(1+\alpha)-\Gamma\right] / 2 R_{b}$} \\
\hline
\end{tabular}

Table C.4: Consumption Allocation in $t=1, c_{1}$, and Liquid Asset Allocation in $t=2, m_{2}$, in Model Cases in Appendix $\mathrm{C}$ 